\title{
Roles of inflammatory signaling and microRNA in the adipose stress response of hibernating Ictidomys tridecemlineatus
}

\author{
Samantha M. Logan
}

B.Sc. Hon. Carleton University, 2015

M.Sc. Carleton University, 2017

A thesis submitted to the Faculty of Graduate Studies and Research in partial fulfilment of the requirements for the degree of:

Doctor of Philosophy

Department of Biology

Carleton University,

Ottawa, Ontario, Canada

(C) Copyright 2021

Samantha Michelle Logan 


\section{Abstract}

Hibernating ground squirrels have an interesting ability to avoid organ dysfunction despite months of obesity, starvation, and low body temperature. However, pro-inflammatory signaling and conserved miRNA expression patterns have yet to be investigated in white and brown adipose tissues (WAT, BAT), organs with roles in fat storage and heat production, respectively. The inflammasome was activated in BAT during torpor and arousal relative to the control, as evidenced by increased inflammasome priming, elevated protein levels of NLRP3, AIM2, cleaved gasdermin D and IL-18, as well as increased caspase-1 activity. By contrast, caspase-1 activity, the ultimate indicator of inflammasome activation, was decreased during torpor and arousal in WAT relative to the euthermic control. Pro-inflammatory cytokines, matrix metalloproteinases (MMPs), and their inhibitors were also investigated to determine if cytokines and tissue remodeling proteins could be important in the stress response in hibernator adipose tissue. An increase in IL-1 $\alpha$ during torpor in BAT furthered the idea that BAT may use proinflammatory pathways as part of the response to cell stress. By contrast, the only change in WAT was a decrease in the total protein levels of MMP2, suggesting tissue remodeling may not be important in the maintenance of WAT homeostasis. Finally, conserved BAT and WAT miRNAs were analyzed. There was an association between the BAT miRNA expression profile and condition (control or torpor), but no association between the two variables in WAT.

Consistently, fewer miRNAs were differentially expressed in WAT than BAT, with more being downregulated than upregulated. As expected, microRNAs were predicted to inhibit energy expensive pathways during torpor in both tissues, suggesting an important role for non-coding RNAs in the regulation of metabolic rate suppression. Unexpectedly, KEGG pathway analysis suggested miRNAs were less likely to target pathways involved in damage sensing and wound repair in BAT, and DNA damage repair in WAT. Together, the data in this thesis suggest an 
upregulation of stress sensing and response in BAT in torpid and arousing ground squirrels through the regulation of inflammasomes, inflammatory signaling, and miRNA expression. By contrast, DNA repair may be increased in WAT but generally, pro-inflammatory pathways were suppressed. 


\section{Statement of Contribution}

I designed and performed all experiments, analyzed the data, created figures, and wrote

all chapters. Dr. Kenneth B. Storey supported all chapters through funding, guiding this research program, editorial review, and final approval of all chapters. Janet M. Storey also provided editorial review. Regarding Chapters 4 and 5, I would like to acknowledge Canada's Michael Smith Genome Sciences Centre, Vancouver, Canada for small RNA-sequencing. A big thank you to Hanane Hadj-Moussa and Liam Hawkins for imparting wisdom regarding initial data processing of miRNA-seq datasets, and Jing Zhang for his assistance troubleshooting R codes used for graphing and statistical analyses.

\section{Chapter 2:}

This chapter has been published in Developmental and Comparative Immunology:

Logan, S.M., and Storey, K.B. (2021). Inflammasome signaling could be used to sense and respond to endogenous damage in brown but not white adipose tissue of a hibernating ground squirrel. Dev. Comp. Immunol. 114, 103819.

The publisher, Elsevier, allows for inclusion of this article, in part or in full, in a thesis or dissertation as part of the rights of the author for personal use.

\section{Chapter 3:}

This chapter has been published in Cellular Signaling:

Logan, S.M. and Storey, K.B. (2021). Markers of tissue remodeling and inflammation in the white and brown adipose tissues of a model hibernator. Cellular Signaling 82: June 2021, 109975. doi:10.1016/j.cellsig.2021.109975

The publisher, Elsevier, allows for inclusion of this article, in part or in full, in a thesis or dissertation as part of the rights of the author for personal use. 


\section{Chapter 4:}

This study has been published in Genomics:

Logan, S.M., and Storey, K.B. (2021). MicroRNA expression patterns in the brown fat of hibernating 13-lined ground squirrels. Genomics 113(2):769-781.

The publisher, Elsevier, allows for inclusion of this article, in part or in full, in a thesis or dissertation as part of the rights of the author for personal use.

\section{Chapter 5:}

At the time of submitting this thesis, this chapter has been submitted for publication and is under peer-review at Molecular Genetics and Genomics.

The publisher, Springer, allows for inclusion of this article, in part or in full, in a thesis or dissertation as part of the rights of the author for personal use. 


\section{Acknowledgements}

Firstly, I would like to thank Dr. Kenneth Storey for accepting me into his lab at a time when I knew nothing about (anything) research and then giving me limitless opportunities to learn, grow, and teach others. I may not have appreciated it at the time, but the moments you were hard on me helped me grow into a more confident and resilient person. Finally, I would not have grown to be the scientist I am today without your guidance, enthusiasm for taking risks, and welltimed encouragement.

Another huge thank you to my committee members, Dr. Iain Lambert and Dr. Matthew Pamenter, who gave me invaluable insights on experimental design and on my career path. Thank you for helping me develop my teaching abilities and diversify my research.

Janet Storey, you never get as much credit as you deserve. Thank you for teaching me practical skills in animal dissection, laboratory management and organization, and strong scientific communication. More importantly, thank you for being a calm and compassionate leader, who was always there to listen to graduate student problems, plan lab outings, and recommend a good book/plant to grow/wine. I hope Ken lets you retire soon. You deserve a break!

I would like to dedicate this thesis to my parents, Jim and Barbara Logan, who instilled in me a strong curiosity for the unknown, a love of reading, and a drive to work hard. Dad, thanks for encouraging me to attend Carleton in the first place, by telling me stories about snakes in the vents, territorial cockroaches, and eccentric Faculty (Ken included) and their research. Thank you also for fixing my computer dozens of times and for lunches spent discussing science and teaching. Mom, thank you for being the most wonderful example of a successful career woman and loving mother. I have a lot to live up to. 
Ryan, thank you for supporting me in following my passions and helping me find balance along the way. I feel incredibly lucky to have found someone who always knew how to keep me grounded and let me fly. I'm excited for the next chapter of our life!

Thank you to Bryan, Christie, Liam, Alex, and Kama for teaching me methods, working alongside me on projects, and being there to discuss everything from science to regular life stuff. To Stuart, thank you for the company on the bus and car rides home from school, the academic banter, and putting up with my teasing - you have been a great friend. Hanane, thanks for spicing up lab meetings with your wry humour (and trolling Ken). You were the "high salt" that neutralized the lab and will have to take the sign from the back room when you leave. Tighe, you have been a great desk buddy but you will never replace Liam as the greatest desk buddy until you start cheering for the right hockey team. To the new generation of Storey lab scientists: in the few years that I have known you, you have made the Storey lab a much better place than you found it. You will make great leaders and scientists. I am glad to have met all of you! 


\section{Table of Contents}

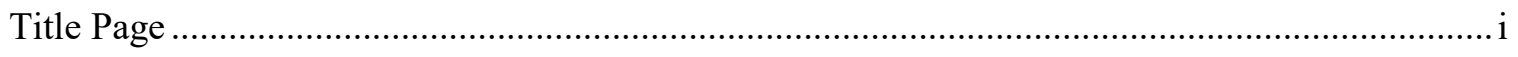

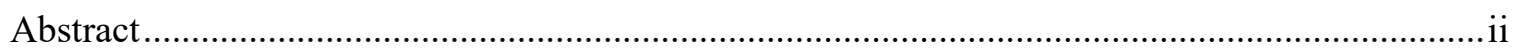

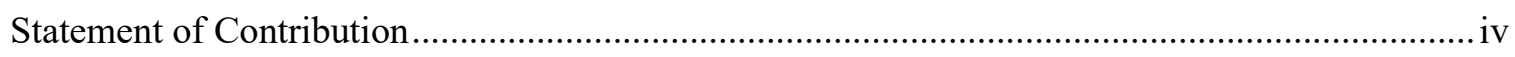

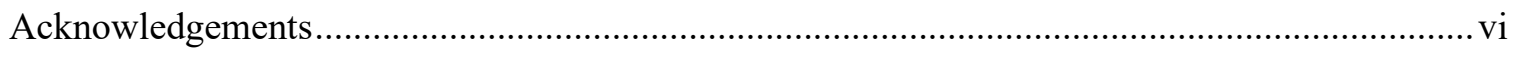

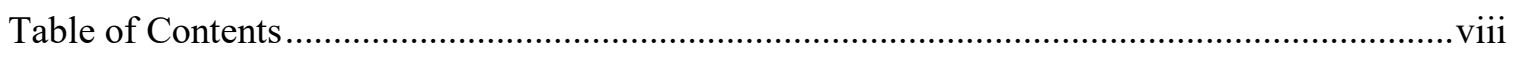

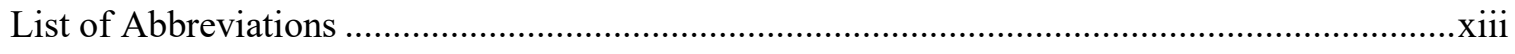

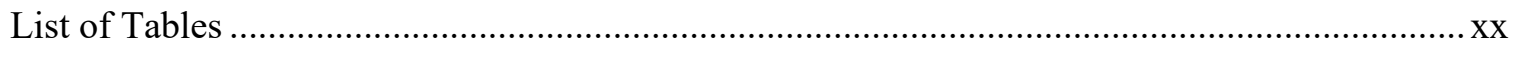

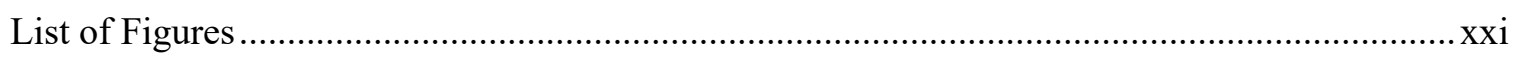

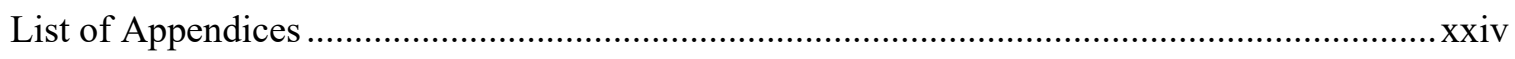

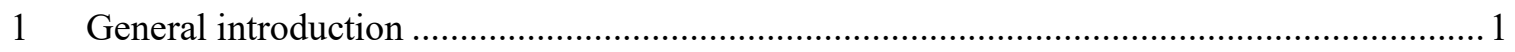

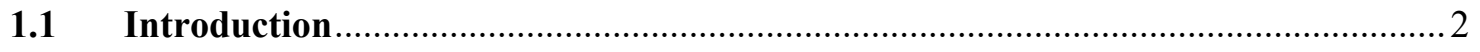

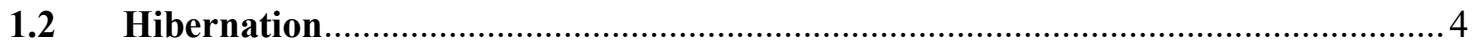

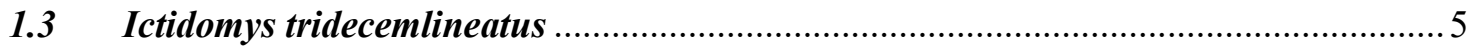

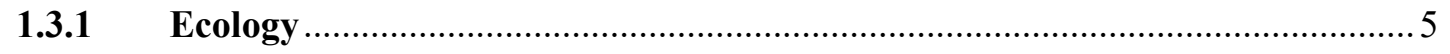

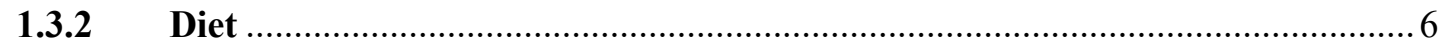

1.3.3 Circannual and torpor-arousal cycles ............................................................. 7

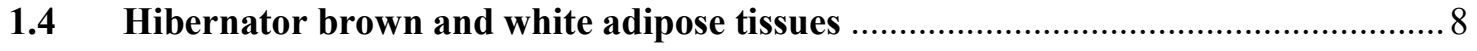

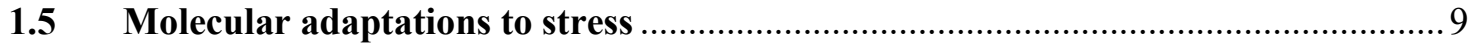

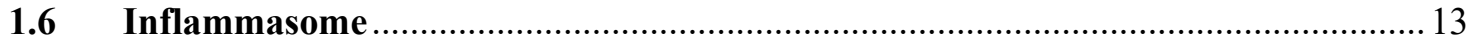

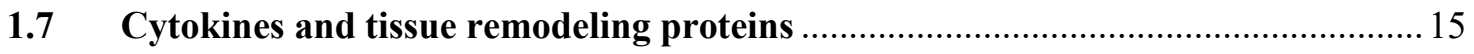

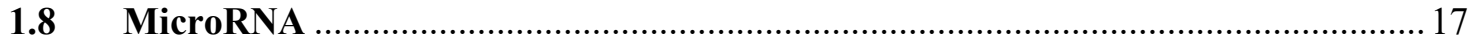

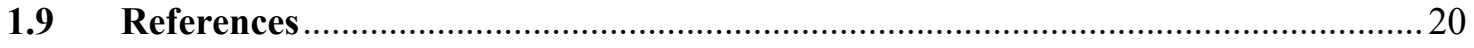

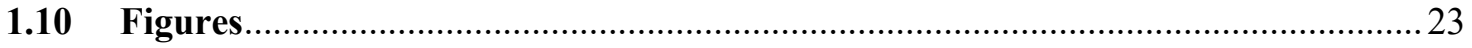

2 Inflammasome signaling could be used to sense and respond to endogenous damage in brown but not white adipose tissue of a hibernating ground squirrel ........................................26

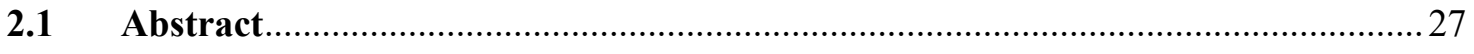

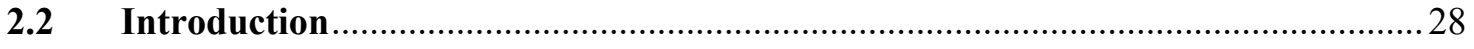

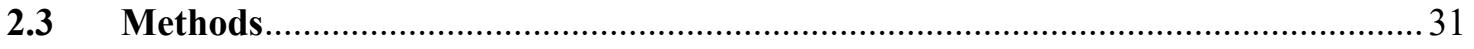

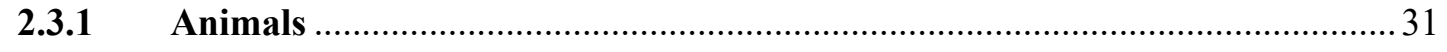

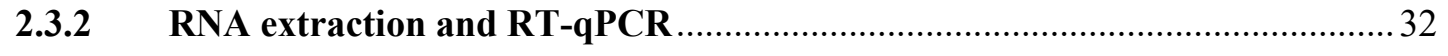

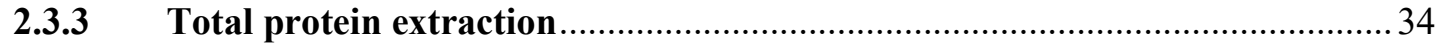

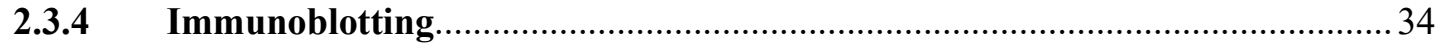

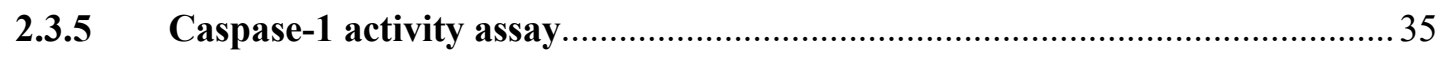




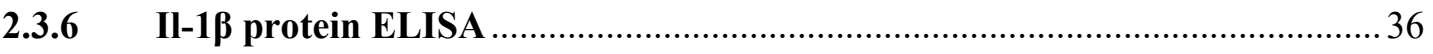

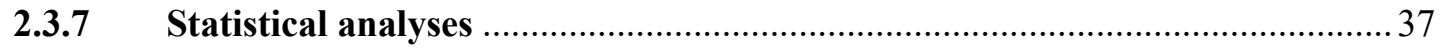

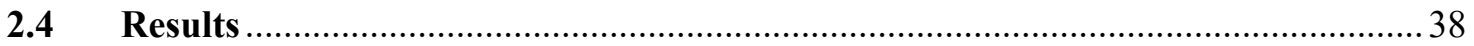

2.4.1 Relative inflammasome transcript levels in brown and white adipose tissue. 38

2.4.2 Western blot analysis of key inflammasome proteins in BAT and WAT ....... 39

2.4.3 Caspase-1 activity is differentially regulated during deep torpor and upon arousal 41

2.4.4 Interleukin 1 beta levels increase in BAT during arousal ..............................42

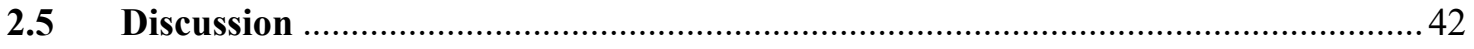

2.5.1 Inflammasome priming events are more evident in BAT than WAT .............4 42

2.5.2 Inflammasome formation more likely occurs in BAT than WAT …...............45

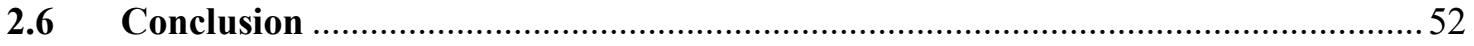

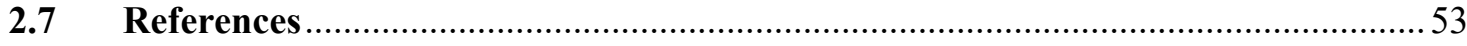

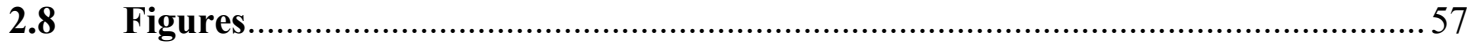

3 Markers of tissue remodeling and inflammation in the white and brown adipose tissues of a

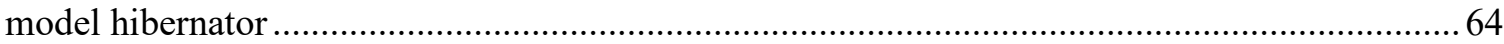

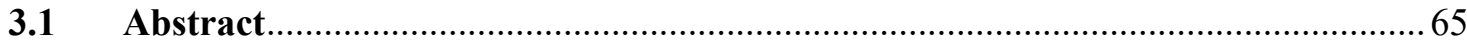

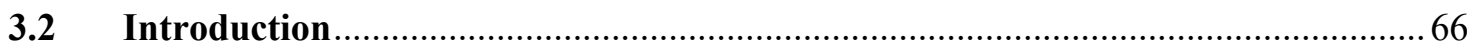

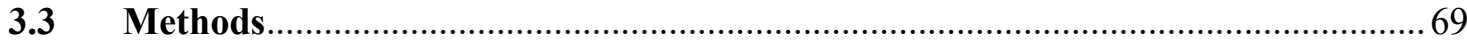

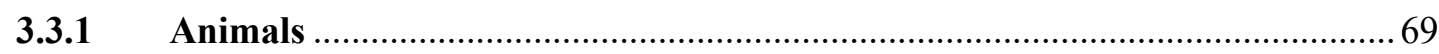

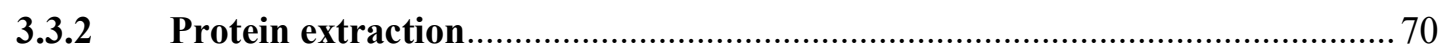

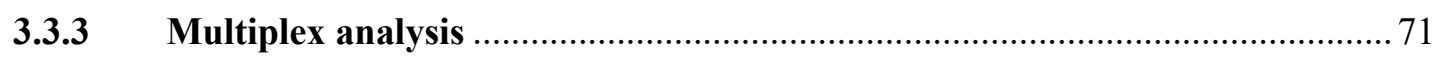

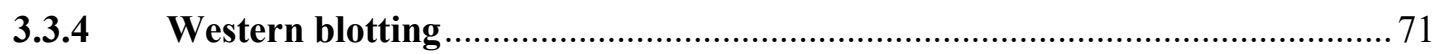

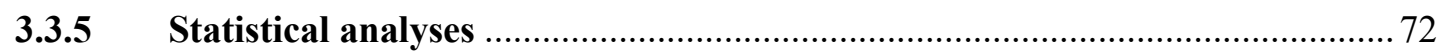

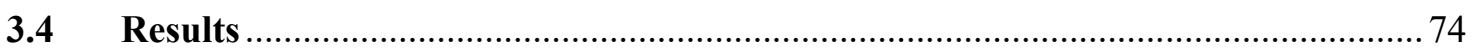

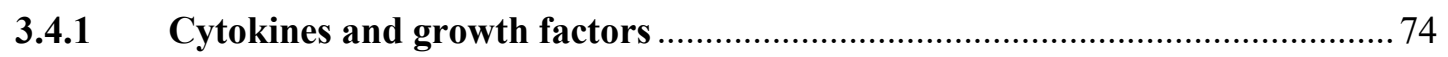

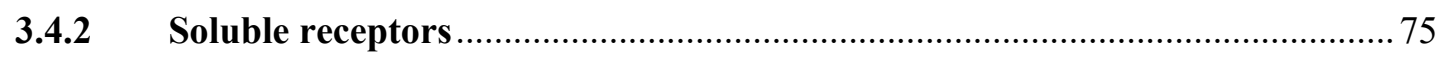

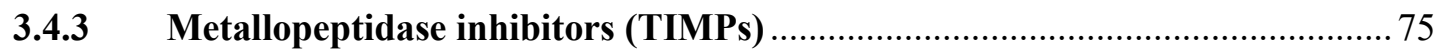

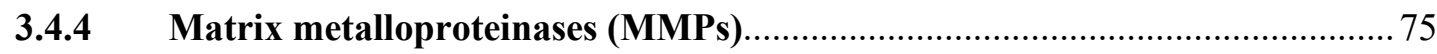

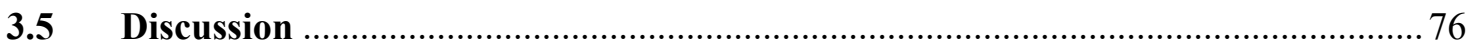

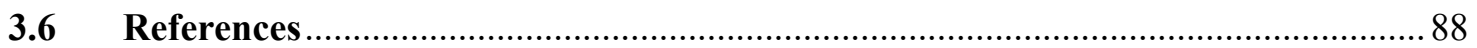

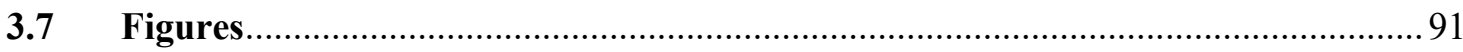

4 MicroRNA expression patterns in the brown fat of hibernating 13-lined ground squirrels... 98

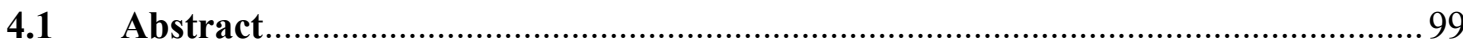

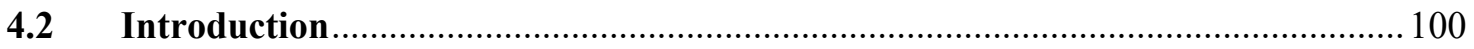




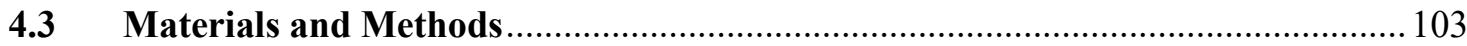

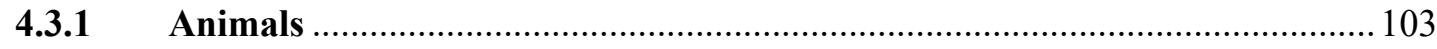

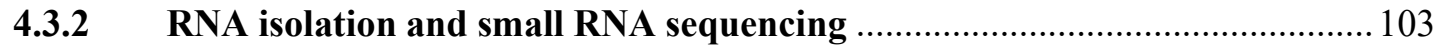

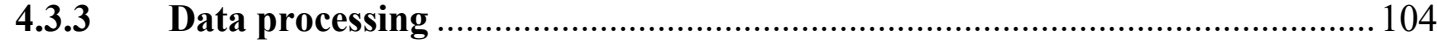

4.3.4 Differential gene expression, supervised and unsupervised cluster analysis 105

4.3.5 Functional characterization of miRNA expression patterns ......................... 106

4.3.6 Random forest feature selection and partial least squares discriminant analysis 107

4.3.7 RNA polyadenylation of miRNA and cDNA synthesis of miRNA ................. 108

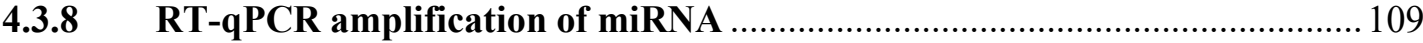

4.3.9 Quantification and statistical analysis of RT-qPCR data ............................. 109

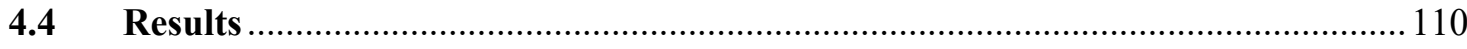

4.4.1 Read counts of conserved miRNAs following small RNA-sequencing analysis 110

4.4.2 Differential gene expression analysis of conserved miRNAs....................... 110

4.4.3 Cluster and principal component analyses ….............................................. 111

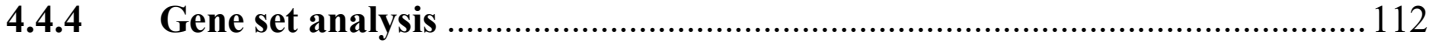

4.4.5 Random forest feature selection and partial least squares discriminant analysis 113

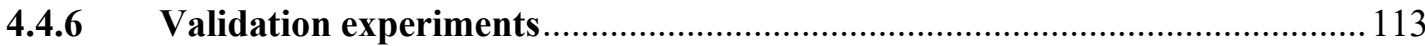

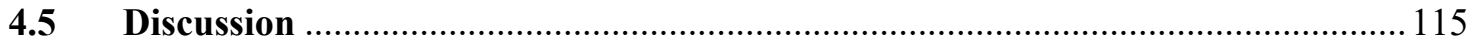

4.5.1 Differential expression of miRNAs during torpor and unsupervised clustering analysis 115

4.5.2 Most miRNAs were significantly upregulated 117

4.5.3 Downregulated miRNAs may regulate cell differentiation and inflammation 122

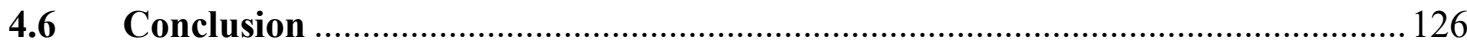

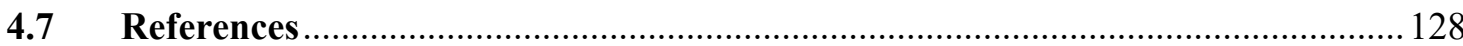

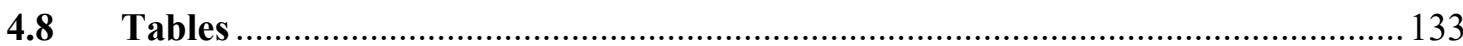

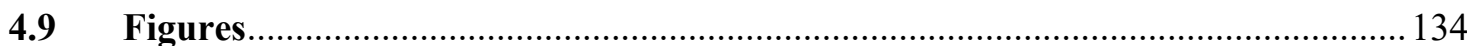

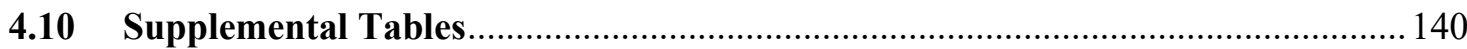

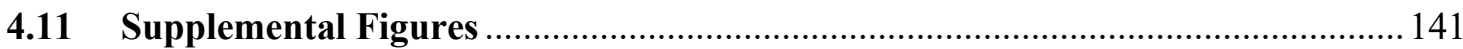

5 MicroRNA regulation of metabolically suppressed white adipose tissue in a wild fat-storing

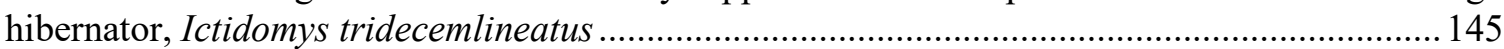

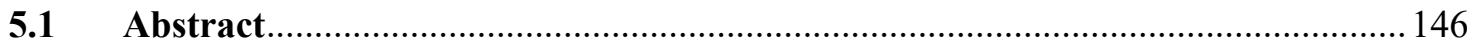

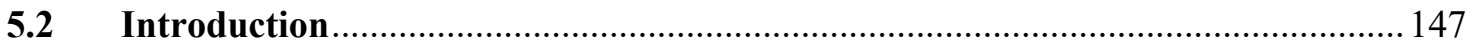




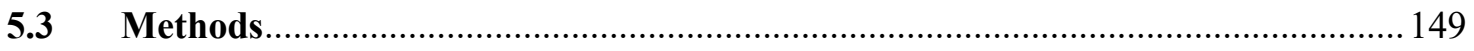

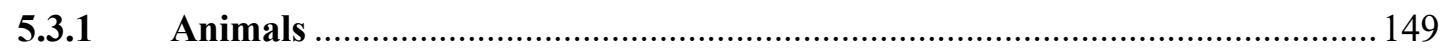

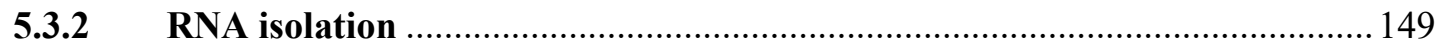

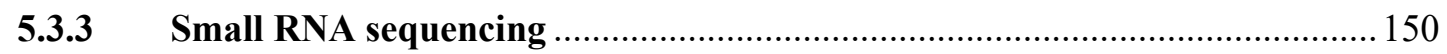

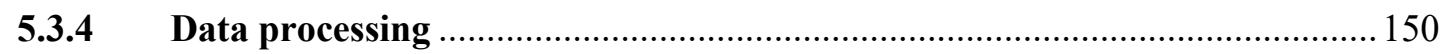

5.3.5 Differential gene expression, supervised and unsupervised clustering analysis 152

5.3.6 Functional characterization of miRNA expression patterns ......................... 153

5.3.7 cDNA synthesis of miRNA and mRNA for RT-qPCR amplification ............. 153

5.3.8 Quantification and statistical analysis of RT-qPCR data ............................. 155

5.3.9 Total protein extraction, Western blotting, and statistical analysis .............. 156

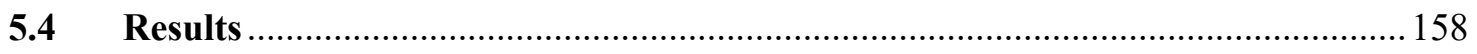

5.4.1 Small RNA-sequencing analysis and differential expression of conserved miRNAs 158

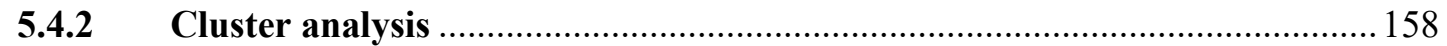

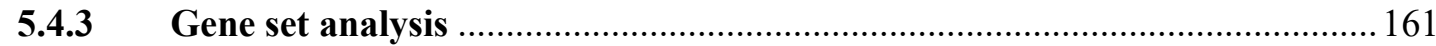

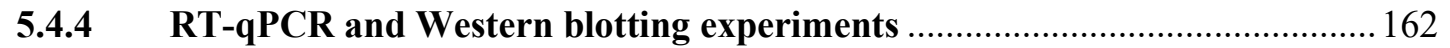

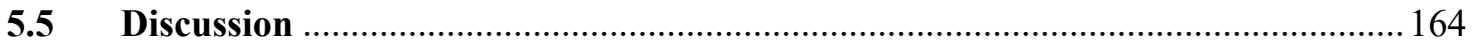

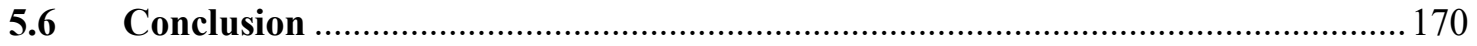

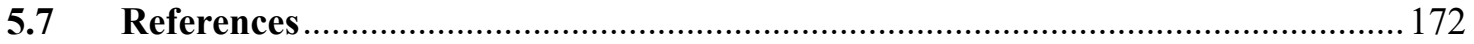

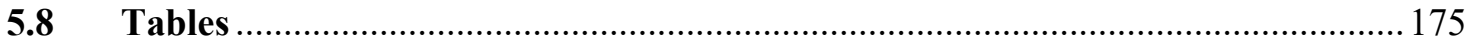

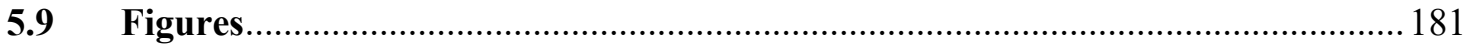

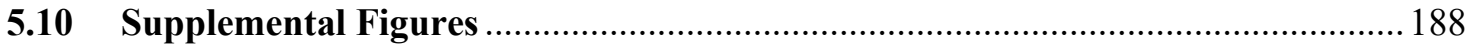

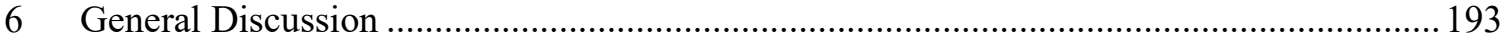

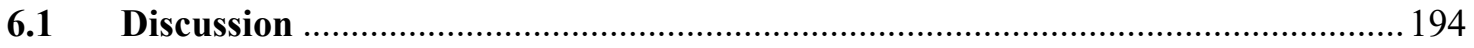

6.1.1 Investigation of the regulation of the inflammasome.................................... 196

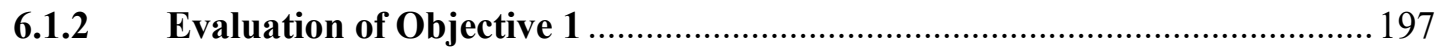

6.2 Differential regulation of pro-inflammatory and tissue remodeling proteins ...... 198

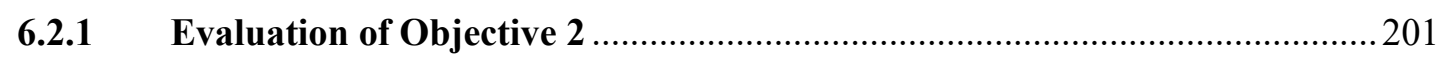

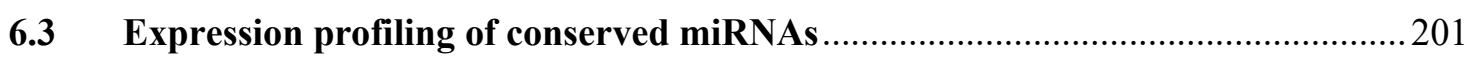

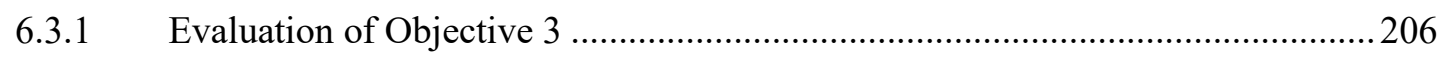

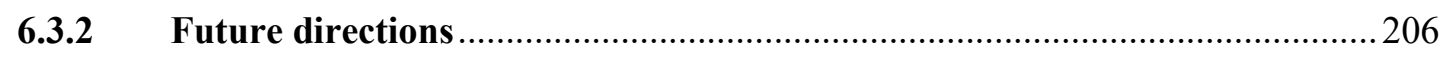

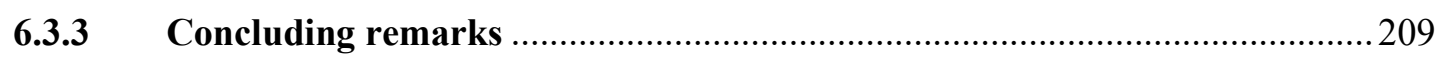

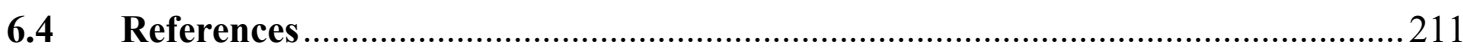

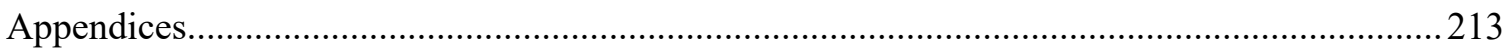




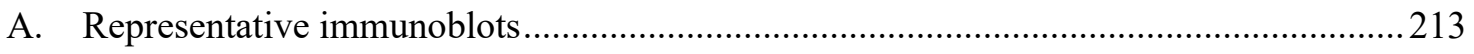

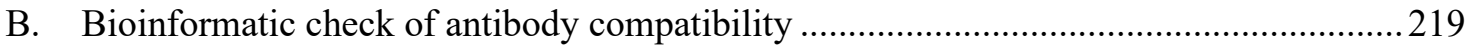

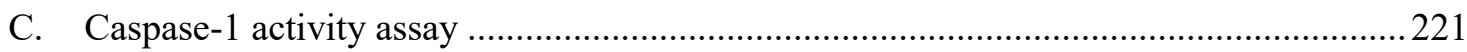

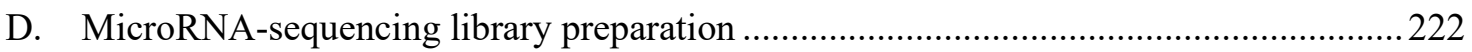

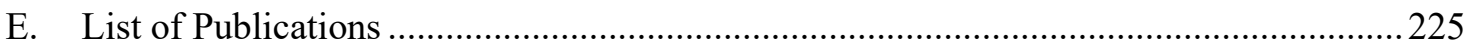

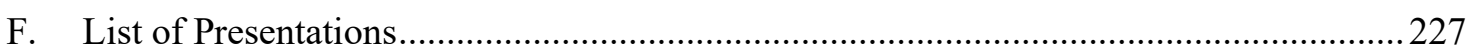




\section{List of Abbreviations}

\begin{tabular}{|c|c|}
\hline Acta & Actin alpha \\
\hline Actb & Actin Beta \\
\hline Actg & Actin gamma \\
\hline ACVRL1 & Activin A receptor like type 1 \\
\hline $\mathrm{AGO} 2$ & Argonaute 2 \\
\hline AIM2 & Absent in melanoma 2 \\
\hline Akt & Protein kinase B \\
\hline ALR & AIM2-like receptor \\
\hline ANKRD52 & Ankyrin Repeat Domain 52 \\
\hline AP-1 & Activator protein 1 \\
\hline ASC & Apoptosis-associated speck-like protein containing a CARD \\
\hline ATF & Activating transcription factor \\
\hline $\mathrm{Bad}$ & BCL2 associated agonist of cell death \\
\hline BAT & Brown adipose tissue \\
\hline Bcl-2 & B-cell lymphoma 2 \\
\hline BER & Base excision repair \\
\hline BMI & Body mass index \\
\hline bpm & Beats per minute \\
\hline BRCA & Breast cancer gene \\
\hline $\mathrm{BRCC} 3$ & BRCA1-BRCA2-containing complex \\
\hline CALM3 & Calmodulin 3 \\
\hline cAMP & Cyclic adenosine monophosphate \\
\hline CARD & Caspase recruitment domain \\
\hline Casp & Caspase \\
\hline CAT & Catalase \\
\hline CCL & Chemokine (C-C motif) ligand \\
\hline
\end{tabular}




\begin{tabular}{|c|c|}
\hline CHK1 & Checkpoint kinase 1 \\
\hline C-IAP & Cellular Inhibitor of Apoptosis Protein 1 \\
\hline CIDEA & Cell death activator \\
\hline CLR & Centered log transformed \\
\hline COP & CARD-only protein \\
\hline COP & Card only protein \\
\hline COX7A & Cytochrome C Oxidase Subunit 7A1 \\
\hline CPT1 & Carnitine palmitoyltransferase \\
\hline CREB & cAMP response element-binding protein \\
\hline CSNK1A1 & Casein Kinase 1 Alpha 1 \\
\hline $\mathrm{Ct}$ & Threshold cycle \\
\hline CXCR & $\alpha$-chemokine receptor \\
\hline $\mathrm{CycB}$ & Cyclin B \\
\hline DAMP & Damage associated molecular pattern molecule \\
\hline DEPC & Diethyl pyrocarbonate \\
\hline DGCR8 & DiGeorge Syndrome Critical Region 8 \\
\hline DNA2 & DNA replication helicase/nuclease 2 \\
\hline DTT & Dithiothreitol \\
\hline EA & Early arousal \\
\hline $\mathrm{EC}$ & Euthermic in the cold room \\
\hline ECL & Enhanced chemiluminescence \\
\hline ECM & Extracellular matrix \\
\hline EGF & Epidermal growth factor \\
\hline EGFR & Epidermal growth factor receptor \\
\hline ELISA & Enzyme-linked immunosorbent assay \\
\hline EN & Entrance into torpor \\
\hline ERK & Extracellular signal-regulated kinase \\
\hline ET & Early torpor \\
\hline
\end{tabular}




\begin{tabular}{|c|c|}
\hline FABP & fatty acid binding protein \\
\hline FDR & False discovery rate \\
\hline FGF21 & Fibroblast growth factor 21 \\
\hline FOXO & Forkhead box transcription factor \\
\hline GNB2 & G protein subunit beta 2 \\
\hline $\mathrm{GO}$ & Gene ontology \\
\hline GO BP & GO biological process \\
\hline GO MF & GO molecular function \\
\hline GSDMD & Gasdermin D \\
\hline GSK 3 Beta & Glycogen synthase kinase 3 beta \\
\hline HMGA2 & High-mobility group AT-hook 2 \\
\hline HMGB1 & High mobility group box protein 1 \\
\hline HSL & Hormone sensitive lipase \\
\hline HSP & Heat shock protein \\
\hline IA & Interbout arousal \\
\hline ICAM-1 & Intercellular adhesion molecule 1 \\
\hline IKK & IאB kinase \\
\hline IL & Interleukin \\
\hline IL-1R & Interleukin 1 receptor \\
\hline IP3 & Inositol 1,4,5-triphosphate \\
\hline IRAK1 & IL-1 receptor-associated kinase 1 \\
\hline IRF & Interferon regulatory factor \\
\hline $\mathrm{I} \kappa \mathrm{B}$ & Inhibitor of nuclear factor kappa B \\
\hline JAK & Janus kinase \\
\hline JNK & c-Jun N-terminal kinase \\
\hline $\mathrm{KCNK} 3$ & Potassium Two Pore Domain Channel Subfamily K Member 3 \\
\hline $\mathrm{kDa}$ & Kilo Dalton \\
\hline KEGG & Kyoto Encyclopedia of Genes and Genomes \\
\hline
\end{tabular}




\begin{tabular}{|c|c|}
\hline LDH & Lactate dehydrogenase \\
\hline LIFR & Leukemia inhibitory factor receptor \\
\hline LIG & DNA Ligase \\
\hline $\operatorname{LogCPM}$ & Log2-counts-per-million \\
\hline LPS & Lipopolysaccharide \\
\hline LRR & Leucine-rich repeat \\
\hline LT & Late torpor \\
\hline MAFB & MAF BZIP Transcription factor B \\
\hline MAPK & Mitogen-activated protein kinase \\
\hline MAVS & Mitochondrial antiviral signaling protein \\
\hline Mcl-1 & Myeloid leukemia cell differentiation protein \\
\hline MCM5 & Minichromosome maintenance complex component 5 \\
\hline MCP & Monocyte-chemotactic protein \\
\hline MDM2 & Mouse double minute 2 homolog \\
\hline MEF2 & Myocyte enhancer factor 2 \\
\hline MFI & Median fluorescent intensity \\
\hline miRNA & MicroRNA \\
\hline MMP & Matrix metalloproteinases/metalloprotease \\
\hline MR & Metabolic rate \\
\hline MRS & Metabolic rate suppression \\
\hline mtDNA & Mitochondrial DNA \\
\hline mTOR & Mechanistic target of rapamycin \\
\hline mtROS & Mitochondrial ROS \\
\hline MYD88 & Myeloid differentiation primary response protein \\
\hline NCBI & National Center for Biotechnology Information \\
\hline NDUFA & NADH dehydrogenase [ubiquinone] 1 alpha subcomplex subunit 9 and 10 \\
\hline NEIL3 & Nei-like DNA glycosylase 3 \\
\hline NEMO & NF-kappa-B essential modulator \\
\hline
\end{tabular}




\begin{tabular}{|c|c|}
\hline NER & Nucleotide excision repair \\
\hline $\mathrm{NF} \kappa \mathrm{B}$ & Nuclear factor kappa-light-chain-enhancer of activated B cells \\
\hline NINDS & National Institute of Neurological Disorders and Stroke \\
\hline NK cell & Natural killer cell \\
\hline NLR & NOD-like receptor/Nucleotide binding leucine rich receptor \\
\hline NLRC4 & NLR Family CARD Domain Containing 4 \\
\hline NLRP & NOD, leucine rich repeat receptor protein \\
\hline NOD & Nucleotide binding oligomerization domain \\
\hline NOS & Nitric oxide synthase \\
\hline NST & Nonshivering thermogenesis \\
\hline $\mathrm{nt}$ & Nucleotide \\
\hline OOB & Out of box \\
\hline ORC & Origin recognition complex \\
\hline PAMP & Pathogen associated molecular pattern molecule \\
\hline PCA & Principal component analysis \\
\hline PCNA & Proliferating cell nuclear antigen \\
\hline PI3K & Phosphoinositide 3-kinase \\
\hline PIP2 & Phosphatidylinositol 4,5-bisphosphate \\
\hline PKA & Protein kinase A \\
\hline PKC & Protein kinase $\mathrm{C}$ \\
\hline PLC gamma & Phospholipase C gamma \\
\hline PLS-DA & Partial least squares discriminant analysis \\
\hline PMSF & Phenylmethylsulfonyl fluoride \\
\hline POP & Pyrin domain-only protein \\
\hline PPARGC1A & Peroxisome proliferator-activated receptor gamma coactivator 1 -alpha \\
\hline $\operatorname{PPAR} \gamma$ & Peroxisome proliferator-activated receptor gamma \\
\hline PPIA & Peptidylprolyl isomerase A \\
\hline PRDM16 & PR domain containing 16 \\
\hline
\end{tabular}




\begin{tabular}{|c|c|}
\hline PRR & Pattern recognition receptor \\
\hline PSMB & Proteasome subunit beta 2 \\
\hline PTM & Post-translational modification \\
\hline PUFA & Polyunsaturated fatty acid \\
\hline PVDF & Polyvinylidene fluoride \\
\hline PYCARD & PYD And CARD Domain Containing \\
\hline PYD & Pyrin domain \\
\hline Raf & Proto-oncogene serine/threonine-protein kinase \\
\hline RAGE & Receptor for advanced glycation end product \\
\hline Ras & Rat sarcoma viral oncoprotein \\
\hline RISC & RNA induced silencing complex \\
\hline RMSEP & Root mean squared error of prediction \\
\hline ROS & Reactive oxygen species \\
\hline sCD30 & Soluble tumor necrosis factor receptor superfamily member 8 \\
\hline SDHA & Succinate dehydrogenase complex flavoprotein subunit A \\
\hline SDS & Sodium dodecyl sulfate \\
\hline sEGFR & Soluble EGFR \\
\hline sgp130 & Soluble Glycoprotein 130/IL6ST \\
\hline SLC25A20 & Carnitine/acylcarnitine translocase \\
\hline SMUG1 & Single-strand-selective monofunctional uracil-DNA glycosylase 1 \\
\hline SOCS & Suppressor of cytokine signaling \\
\hline SOD & Superoxide dismutase \\
\hline STAT & Signal transducer and activator of transcription \\
\hline TAB & TAK1 binding protein \\
\hline TAK1 & TGF $\beta$-activated kinase 1 \\
\hline $\mathrm{Tb}$ & Body temperature \\
\hline TBARS & Thiobarbituric acid reactive substances \\
\hline Tbp & TATA-binding protein \\
\hline
\end{tabular}


TBST

TCA

TGFBR2

TGF- $\beta$

TIMP

TLR

TMB

Tmet

TNFR

$\mathrm{TNF} \alpha$

TRAF

TRX

Tset

TXNIP

UCP1

UPR

VCAM-1

VEGF-A

VEGFR

WAT

WNT

X-IAP
Tris-buffered saline with Tween-20

Tricarbocxylic acid cycle

Transforming growth factor beta receptor 2

Transforming growth factor beta

Tissue inhibitor of metalloproteinases

Toll-like receptors

3,3',5,5'-tetramethylbenzidine

T-methionine tRNA

Tumor necrosis factor receptor

Tumor necrosis factor

TNF receptor associated factor

Thioredoxin

Thermogenic set-point

Thioredoxin interacting protein

Uncoupling protein 1

Unfolded protein response

Vascular cell adhesion protein 1

Vascular endothelial growth factor A

Vascular endothelial growth factor receptor

White adipose tissue

Wingless and int-1

X-Linked Inhibitor of Apoptosis Protein 


\section{List of Tables}

Table 4.1: Summary of Pearson correlation coefficients, $p$-values and FDR-adjusted p-values for positively correlated miRNAs of interest, miRNAs 1-3p, 206, 133a-3p, and 361-3p

Table 5.1: Primers used in RT-qPCR analysis of miRNAs................................................. 175

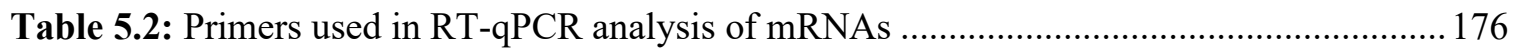

Table 5.3: Differential expression of miRNAs using a p-value of 0.05 as the threshold for significance. Log2-transformed fold-changes in gene expression and false discovery rate-adjusted p-values are also shown for the differentially expressed miRNAs. .......................................... 177

Table 5.4: Significantly enriched KEGG pathways ........................................................... 178

Table 5.5: Ten most positively and negatively enriched GO biological processes, out of 1033 significantly enriched GO biological processes.

Table 5.6: Ten most positively and negatively enriched GO molecular function, out of 144 significantly enriched GO biological processes. 180

Supplemental Table 4.1: Differential expression of miRNAs using $\mathrm{q}<0.05$ as the threshold for significance.

Supplemental Table 4.2: Highly significant Pearson correlation clusters of miRNAs $(q<0.01)$ for control and torpor BAT samples.

Supplemental Table 4.3: Significant Pearson correlation clusters of miRNAs $(q<0.5)$ for control and torpor samples......

Supplemental Table 4.4: Experimentally validated interactions between all miRNAs and mRNAs.

Supplemental Table 4.5: KEGG, GO biological process, and GO molecular function analyses.

Supplemental Table 4.6: Random forest feature selection results. 140

Supplemental Table 4.7: PLS-DA Permutation test results, showing RMSEP values for all 999 iterations for both control and stress comparisons.

Supplemental Table 4.8: Primers used in RT-qPCR analyses of miRNAs. 140 


\section{List of Figures}

Figure 1.1: 13-lined ground squirrel picture and national and state/province distribution.

Figure 1.2: Torpor-arousal cycle of a hibernating ground squirre.

Figure 1.3: Summary of the expression patterns of some antioxidants, protein folding chaperones, apoptotic proteins, damage associated molecular pattern molecules and stress inducible signaling proteins in the brown adipose and white adipose tissues.

Figure 2.1: Relative transcript levels of key inflammasome targets using brown adipose tissue (BAT) sampled from 13-lined ground squirrels at 6 different points of the torpor-arousal cycle. 57

Figure 2.2: Relative transcript levels of seven key inflammasome pathway components in the white adipose tissue sampled from ground squirrels hibernating at 6 points of the torpor-arousal cycle

Figure 2.3: Inflammasome protein abundance patterns in brown adipose tissue (BAT) at 6 points of the torpor-arousal cycle. .59

Figure 2.4: Inflammasome protein abundance patterns in white adipose tissue (WAT) at 6 points of the torpor-arousal cycle.

Figure 2.5: Caspase-1 activity (units) in BAT and WAT total protein homogenates from 13-lined ground squirrels sampled at three time points of the torpor-arousal cycle.

Figure 2.6: Interleukin-1 $\beta$ (IL-1 $\beta$ ) concentration $(\mathrm{pg} / \mathrm{mL})$ in BAT and WAT total protein homogenates from 13-lined ground squirrels sampled at three time points of the torpor-arousal cycle.

Figure 3.1: General signal transduction pathways initiated by selected cytokines (in bold) and their receptors.

Figure 3.2: Matrix metalloproteinase (MMP) activation and substrates

Figure 3.3: Relative fluorescence intensities of cytokine and growth factor analytes in A) BAT and B) WAT from 13-lined ground squirrels, assessed via multiplex assay.

Figure 3.4: Relative fluorescence intensities of soluble receptors in A) BAT and B) WAT from 13-lined ground squirrels, assessed via multiplex assay.

Figure 3.5: Relative fluorescence intensities of TIMP1-4 in A) BAT and B) WAT from 13-lined ground squirrels, assessed via multiplex assay

Figure 3.6: Relative protein levels of matrix metalloproteinases (MMPs) in ground squirrel BAT, assessed via western blot 
Figure 3.7: Relative protein levels of matrix metalloproteinases (MMPs) in ground squirrel WAT, assessed via western blot

Figure 4.1: Volcano plot and supervised cluster analysis of differentially abundant microRNA in 13-lined ground squirrel BAT considering fold changes $>1.5$ and $\mathrm{q}<0.01$

Figure 4.2: Analysis of trends in the complete miRNA dataset using a heatmap of the unsupervised cluster analysis and principal component analysis 135

Figure 4.3: Supervised Pearson correlation heatmap (A) and correlation plot (B) of differentially abundant microRNAs in BAT from 13-lined ground squirrel sampled during control (EC) conditions 136

Figure 4.4: Supervised Pearson correlation heatmap (A) and correlation plot (B) of differentially abundant microRNAs in BAT from 13-lined ground squirrel sampled during torpor (LT) conditions

Figure 4.5: Gene set analysis of global miRNA expression patterns using volcano plots to show $\log 2$ transformed model coefficients and their distribution with respect to the $\log 10 \mathrm{p}$-value for KEGG Pathways and GO biological processes.

Figure 4.6: RT-qPCR validation of the relative levels of miRNAs in BAT.

Figure 5.1: Volcano plot and supervised cluster analysis of enriched microRNAs from LT 13lined ground squirrel WAT, compared to EC

Figure 5.2: Results of unsupervised clustering analysis and principle component analysis (PCA) of samples based on relative WAT miRNA levels comparing EC and LT.

Figure 5.3: Supervised Pearson correlation plots of differentially expressed microRNAs ( $\mathrm{p}<$ 0.05 ), showing miRNAs that are co-expressed during in WAT of euthermic in the cold room control animals (EC)

Figure 5.4: Supervised Pearson correlation plots of differentially expressed microRNAs ( $\mathrm{p}<$ 0.05 ), showing miRNAs that are co-expressed during torpor (LT).

Figure 5.5: Significantly enriched KEGG pathways in WAT.

Figure 5.6: RT-qPCR of miRNA-seq and downstream target genes in 13-lined ground squirrel WAT, comparing EC and LT. 186

Figure 5.7: Relative protein levels of $\mathrm{p} 53$ protein and $\mathrm{p} 53$ phosphorylation markers in white adipose tissue comparing EC and LT ground squirrels ..... 
Supplemental Figure 4.1: Pathview analysis of significantly enriched and miRNA-inhibited pathways during torpor, highlighing A) ribosome, B) glycolysis and gluconeogenesis and C) oxidative phosphorylation.

Supplemental Figure 4.2: Pathview analysis of significantly enriched and less inhibited KEGG pathways during torpor, highlighting $\backslash$ ) Cytokine-cytokine receptor interactions and B) complement and coagulation cascades

Supplemental Figure 4.3: Random forest feature selection and principal component analysis of resulting genes

Supplemental Figure 4.4: PLS-DA model A) permutation test and b) final model using one component. C) summary of the permutation test, where $\mathrm{p}<0.05$ indicates the model can reliably classify samples as either control or torpor using the subset of 64 miRNA

Supplemental Figure 5.1: Significantly enriched GO biological processes 188

Supplemental Figure 5.2: Significantly enriched GO molecular functions. 189

Supplemental Figure 5.3: Pathview analysis of Base Excision Repair KEGG Pathway ..... 190

Supplemental Figure 5.4: Pathview analysis of p53 signaling pathway 191

Supplemental Figure 5.5: Pathview analysis of NOD-like receptor signaling pathway 192 


\section{List of Appendices}

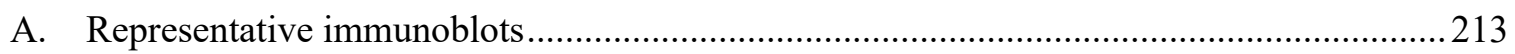

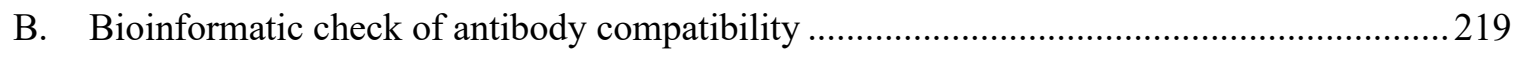

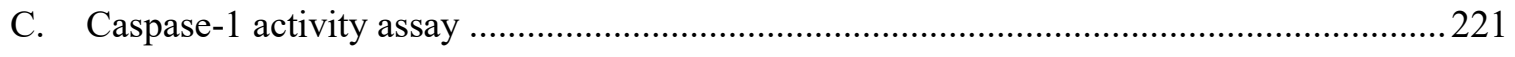

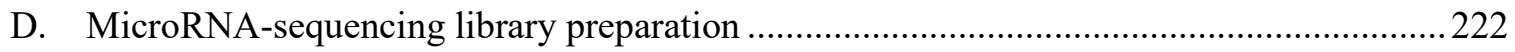

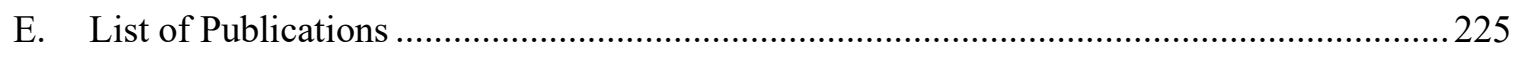

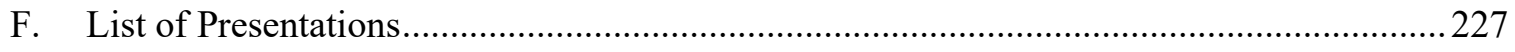




\section{General introduction}




\subsection{Introduction}

There are places on this planet that would reasonably be believed to be uninhabitable, especially by certain species, and yet, in the words of Ian Malcolm from the movie Jurassic Park, "Life, uh... finds a way". While some species of birds and insects may migrate south to avoid the winter conditions that make finding food and escaping predators difficult, other animals adapt to their local surroundings instead. Adaptation can come in many forms. Endothermic species that remain active during the winter season may grow a thick fur coat in colours that hide them from predators, while others may hoard food or convert food into large body adipose reserves for a steady supply of nutrition until spring. However, for many species, it is too energetically expensive to migrate or maintain a high body temperature throughout the winter. Ectothermic species, such as the red-eared slider turtle (Trachemys scripta elegans) and painted turtle (Chrysemys picta), can survive for up to 4.5 months at the bottom of ice-covered freshwater ponds with little to no oxygen availability. Similarly, winter for the wood frog (Rana sylvatica) means freezing solid while staying hidden under leaf litter of the forest floor. Some endothermic species also decrease their core body temperature and metabolic rate, while remaining immobile for months at a time in a state of hibernation. Indeed, certain species of ground squirrel, prairie dog, and marmot survive for over six months without eating, hunkered down in underground burrows. So how do they do it? The key is metabolic suppression.

Adaptation to inhospitable environments by suppressing metabolic rate and greatly reducing normal physiological processes allows capable organisms to save energy while in stasis until the requirements for active life are available again (e.g. food, water, oxygen, etc.). In such hypometabolic states, ATP consumption associated with physiological processes such as movement, breathing rate, heart rate, nutrient absorption, digestion, blood filtration, and defending a high body temperature $\left(\mathrm{T}_{\mathrm{b}}\right)$ is nearly eliminated as part of coordinated metabolic rate depression. Importantly, torpor is defined as a state of inactivity that is characterized by a 
decrease in metabolic rate and/or $\mathrm{T}_{\mathrm{b}}$. Torpor can be used by animals facing a wide range of environmental stressors and is not specific to a season, unlike hibernation and aestivation, which refer to torpor used to adapt to inhospitable conditions during winter and summer, respectively.

Each species that is capable of using torpor employs molecular mechanisms to adapt to unique and dynamic environmental stressors. Consistent across species capable of torpor is the suppression of gene expression except for the genes that ensure proper cell functioning and tissue viability (Storey and Storey, 2005). Most protein products are protected from degradation, and there are increases in the expression of molecular chaperones, pro-survival proteins, and antioxidants (Rouble et al., 2014, 2013). Organisms capable of entering hypometabolic states regulate gene expression at a variety of levels. Epigenetic markers on histone proteins and DNA help to control which genes are transcribed by transcription factors, and the translated proteins are also regulated in terms of total protein level and post-translational modifications (PTMs) (Storey, 2015; Storey et al., 2010). RNA-binding proteins have been proposed to regulate transcript viability, translocation to the cytoplasm, and storage in stress granules for future translation (Storey, 2015; Storey and Storey, 2005). New data also demonstrates a key role for microRNAs in the post-transcriptional regulation of transcripts, since microRNAs have the potential to regulate all processes in the cell through complementary sequence binding, transcript sequestration, or transcript degradation (Storey, 2015). Translation factors are also reversibly phosphorylated to regulate their activity, as are many other proteins in the cell (Storey and Storey, 2005). Finally, enzyme activity is regulated by the presence or absence of substrates, cofactors and reversible PTMs, as well as by changes in temperature (Storey and Storey, 2005). Altogether, metabolic rate suppression involves carefully coordinated molecular adaptations that match energy expenditure with oxygen, ATP, and nutrient availability and promote tissue homeostasis. 


\subsection{Hibernation}

Hibernation is a special form of torpor that is used by mammals that adapt to winter conditions by avoiding active life until conditions are more suitable for their success. Hibernation and torpor in general have been studied since as early as the mid-1500s (Withers and Cooper, 2010). Hibernation and aestivation (summer torpor) are forms of dormancy, where metabolism is greatly reduced but still apparent. Cryptobiosis, on the other hand, refers a the state of "suspended animation" where organisms appear to have no metabolism at all, often due to complete desiccation or the absence of cellular oxygen (Withers and Cooper, 2010). Hibernation involves a substantial suppression of metabolic rate (MR) and the abandonment of homeothermy. Some mammals like arctic ground squirrels take this to the extreme, allowing their $\mathrm{T}_{\mathrm{b}}$ to fall as low as $-2.9^{\circ} \mathrm{C}$, but other hibernators like black bears can only decrease their $\mathrm{T}_{\mathrm{b}}$ by a few degrees due to their large size and extensive thermal insulation by fur and large adipose reserves. Metabolic rate and core $\mathrm{T}_{\mathrm{b}}$ are intertwined since a decrease in MR reduces the amount of cellular energy dissipated as heat. Further, a decrease in core $\mathrm{T}_{\mathrm{b}}$ facilitates a reduction in MR since most mammalian enzymes operate at optimum temperatures of $37^{\circ} \mathrm{C}$.

Hibernation is used by a range of animals in the class Mammalia including monotremes like the echidna (Tachyglossus aculeatus), marsupials like the pygmy possum (Cercartetus spp.), and placental mammals such as jumping mice (Zapus spp.), ground squirrels (Spermophilus spp.), marmots (Marmota spp.), bears (e.g. Ursus spp.), and bats (e.g. Myotis spp.), among others (Geiser, 2004). Hibernation can be obligate, meaning that they hibernate in a predictable circannual pattern or facultative, meaning that they use torpor to survive extreme environmental challenges as they arise, irrespective of the season. Hibernators can also be fat-storing like marmots, ground squirrels, and brown bears or food-storing like chipmunks, hamsters, and pocket mice. 
Species that use hibernation can be differentiated from species that use daily torpor based on the depth and duration of torpor, but there are species that can use daily torpor and longer torpor bouts. Hibernators use multi-day torpor-bouts typically spanning a week or more and species that use daily torpor typically only do so to avoid a particular daily stress (e.g. changes in temperature or predation) for 3-12 hours (Ruf and Geiser, 2015). Hibernators typically show greater suppression of metabolic rate and body temperature than species that use daily torpor. Finally, most hibernators use interbout arousals (e.g. ground squirrels, marmots, bats) throughout the hibernation months but hibernation can also involve one continuous torpor bout (e.g. black bears). Periodic arousals back to euthermic body temperature $\left(37^{\circ} \mathrm{C}\right)$ are energetically expensive but appear to be necessary to get restful sleep (restoring neuronal balance) and remove potentially toxic metabolic by-products.

\subsection{Ictidomys tridecemlineatus}

The 13-lined ground squirrel, Ictidomys tridecemlineatus, previously called Citellus tridecemlineatus and then Spermophilus tridecemlineatus, was originally identified in 1821 in Minnesota, U.S.A. (Burnett, 1914). This species is a member of the Family Sciuridae under the Order Rodentia. It is one of the most studied obligate and fat-storing hibernators. This ground squirrel is a good model organism for hibernation studies for several reasons, including the availability of a sequenced genome and computationally predicted protein sequences, as well as a strong understanding of its physiology, diet, and circannual hibernation patterns.

\subsubsection{Ecology}

I. tridecemlineatus inhabits the plains of three Canadian provinces (Alberta, Saskatchewan and Manitoba) and a range of states in the U.S.A including as far west as Montana, Utah and Arizona, and as far east as Pennsylvania (Figure 1.1). Regardless of where they occur in their range (Manitoba down to Texas), I. tridecemlineatus is an obligate hibernator (Kisser and 
Goodwin, 2012). They hibernate in burrows that are only as deep as they need to be. In fact, burrow length is quite short $(0.2 \mathrm{~m})$ relative to other burrowing rodent species, and burrow length is proportional to their body size and not temperature stress (Van Vuren and Ordeñana, 2012).

\subsubsection{Diet}

Most ground squirrels in North America consume plant matter as a large part of their diet, including fruit, roots, seeds, and greens, but almost all are known to also eat other animals including adult and larval insects, field mice, eggs, and baby birds (Burnett, 1914; Helgen et al., 2009). I. tridecemlineatus is an agricultural pest since it eats grain (including corn and barley), and freshly sown seeds of any kind, but if grasshoppers are available (another agricultural pest), they will feed on grasshoppers almost exclusively (Burnett, 1914; Hisaw and Emery, 1927). The development of ground squirrel colonies for hibernation experiments was facilitated by exchanging a rodent chow (23\% protein) for a high-protein dog or cat chow (26-30\% protein) (Merriman et al., 2012; Vaughan et al., 2006). Specifically, this ensured the ground squirrels had enough protein in their diet which lessened the cases of maternal cannibalism and fatalities during hibernation (Vaughan et al., 2006).

Polyunsaturated fatty acids (PUFAs) are important in maintaining membrane fluidity of cells at the low body temperatures experienced during torpor, but they cannot be synthesized de novo by mammals and, therefore, wild hibernators must carefully select foods that contain optimal levels of this key nutrient (Frank and Storey, 1995). Two PUFAs of particular importance in hibernation are omega-3 alpha-linolenic acid $(18: 3$ cis-9,12,15) and omega-6 linoleic acid $(18: 2$ cis-9,12). Consuming diets that are low in omega-6 essential PUFAs is correlated with ground squirrels spending less time in torpor, and in shallower torpor bouts (with higher $\mathrm{T}_{\mathrm{b}}$ ). However, increased levels of PUFAs can be dangerous given their proneness to autooxidation, resulting in lipid peroxidation and subsequent tissue damage (Frank and Storey, 1995). 


\subsubsection{Circannual and torpor-arousal cycles}

Some 13-lined ground squirrels can enter hibernation as early as July, which is what some male ground squirrels do post-reproduction. However, females must endure a gestation period of approximately one month and wait 5 weeks until the young are weaned before the female and her young can begin fattening for winter torpor (Burnett, 1914; Kisser and Goodwin, 2012; Vaughan et al., 2006). Therefore, typically 13-lined ground squirrels enter torpor between October and early November where males enter torpor first, followed by adult females and then juvenile ground squirrels (Burnett, 1914; Kisser and Goodwin, 2012) (Figure 1.2).

During hibernation, ground squirrels suppress their metabolic rate by more than $95 \%$ and they become heterothermic, allowing their body temperature to decline from $37^{\circ} \mathrm{C}$ to low temperatures between $0-10{ }^{\circ} \mathrm{C}$, depending on burrow temperature. Physiological parameters such as breathing rate, heart rate, and organ perfusion also decrease during deep torpor (Storey and Storey, 2005). Hibernating ground squirrels have been shown to have apnoic breathing, where in general, breathing rate decreases from $\sim 100-200$ breaths/min to about 5 breaths/min (Cooper et al., 2016). More specifically, 13-lined ground squirrels decrease their breathing rate from approximately 40 breaths/min to less than 1 breath/minute, and also show episodic breathing (1-2 sharp breaths followed by long exhales) (Landau and Dawe, 1958). Heart rate decreases from 300-400 bpm to 5-10 bpm, and blood pressure falls from 135/90 to 80/40 $\mathrm{mmHg}$ (Cooper et al., 2016).

Arousals require sharp increases in metabolic rate to generate the heat needed to return to $\sim 37^{\circ} \mathrm{C}$ body temperature. As such, squirrels may use $70-80 \%$ of their energy budget for the entire hibernation season during interbout arousals (Grabek et al., 2015). Ground squirrels can bring their body temperature up to normal temperatures within 3 hours. During the arousal period, breathing and heart rates increase to $\sim 200$ breaths/minute and $\sim 400$ beats/min, respectively, before settling back down to resting breathing and heart rates of 100-125 breaths/min and 200- 
250 beats/minute. Arousal periods can last up to 2 days before animals descend back into torpor.

There may be 10-20 torpor-arousal bouts until the final spring arousal, which occurs between March and April (Burnett, 1914; McCarley, 1966) (Figure 1.2).

\subsection{Hibernator brown and white adipose tissues}

Brown and white adipose tissues, referred herein as BAT and WAT, respectively, have important roles in ensuring the survivability of hibernators. Brown adipocytes have many mitochondria and multilocular lipid droplets, meaning many smaller sized lipid droplets, compared to white adipocytes which typically contain one large lipid droplet and only several mitochondria in the cytoplasm.

BAT matures prenatally and is important for non-shivering thermogenesis, whereas WAT typically matures postnatally and is important in lipid storage. In hibernators, both adipose depots grow proportionately with total body mass in the fall, due to increases in both adipocyte size (hypertrophy) and number (hyperplasia), while the mass of fat-storing hibernators like ground squirrels nearly doubles relative to the weight of summer ground squirrels. Of the total body weight of a pre-hibernation ground squirrel, $60 \%$ of the mass is associated with WAT stores and another 2\% corresponds to BAT depots (MacCannell et al., 2018). WAT stores triglycerides, which are the primary source of cellular energy for most organs during torpor (Storey and Storey, 2005). Triglycerides released from WAT are transported to the liver where they are converted into ketone bodies (acetone and beta-hydroxybutryate) via beta-oxidation and are then released back into circulation for use as fuels by organs such as the brain. Additionally, glycerol from triglyceride hydrolysis is converted into glucose by the liver, and exported for use by organs such as the brain (Storey and Storey, 2005). Brain is one of the few organs that cannot transport triglycerides into their mitochondria for ketogenesis, due to a lack of or low expression of 
carnitine palmitoyltransferase (CPT1), and so the brain relies on the important functions of WAT and liver to sustain homeostasis.

Triglycerides that come from WAT as well as internally stored lipids are used to fuel non-shivering thermogenesis in BAT (Hittel and Storey, 2001; Nedergaard and Cannon, 1984). Non-shivering thermogenesis is required for the rewarming of ground squirrels in the initial stages of arousal to euthermia. Briefly, sympathetic input from the brain in the form of norepinephrine triggers $\beta 3$ adrenergic receptors in BAT to activate adenylyl cyclase, which increases the production of cyclic AMP (Cannon and Nedergaard, 2004). Elevated cAMP levels activate protein kinase A (PKA), which then phosphorylates perilipin, leading to its detachment from triglycerides. PKA also phosphorylates hormone sensitive lipase (HSL) to activate it: HSL promotes lipolysis of the freed triglycerides into fatty acids. Most free fatty acid molecules are shuttled into the mitochondria via the carnitine transport system where they are metabolised via beta-oxidation into acetyl-coA that is then processed via the TCA cycle to produce $\mathrm{CO}_{2}$ and $\mathrm{NADH} / \mathrm{FADH}_{2}$ reducing equivalents important for the generation of a proton gradient in the intermembrane space. Instead of using the potential energy of the proton gradient to generate ATP, brown adipocytes dissipate the proton gradient to generate heat via the action of uncoupling protein 1 (UCP1). It has been said that BAT is essential for interbout arousals to euthermia in ground squirrels, since shivering thermogenesis by skeletal muscle does not activate until the ground squirrel has warmed to approximately $15^{\circ} \mathrm{C}$.

\subsection{Molecular adaptations to stress}

Hibernators have an interesting history of not responding to physical damage or pathogenic insult while in the throes of torpor. One study demonstrated that hibernating 13-lined ground squirrels cut on their hindlimbs during torpor do not bleed until arousal, when heart rate, blood temperature, and blood pressure are increased (Landau and Dawe, 1958). Hibernating 
ground squirrels infected with gastrointestinal roundworms do not produce antibodies against the subcutaneously injected parasites, and only slightly increase antibody production upon arousal (Cahill et al., 1967). Despite this, very few roundworms survive. Similarly, ground squirrels injected with whole blood from other animals or given skin transplants do not produce an immune response until arousal. Hibernating animals have fewer circulating immune cells than do euthermic controls, suggesting sequestration of immune cells during torpor (Bouma et al., 2010). Much research has been done to demonstrate that hibernators shut off their adaptive immune system during torpor, but very few studies have investigated markers of innate immune system activation.

The innate immune system is the body's first line of defence against pathogens and tissue damage and is non-specific to the threat. For example, skin and mucous membranes function as physical barriers against all viruses or bacteria. Another component of the innate immune system is the complement system whose role is to enhance the function of antibodies and phagocytic cells by crippling pathogens (e.g. slowing down and identifying bacteria for phagocytosis) and recruiting phagocytic cells to the site of infection. Phagocytic white blood cells (leukocytes) may engulf foreign material. Natural killer cells (NK cells) may recognise cells whose surface markers (specifically the presence of major histocompatibility complex I) have changed (e.g. infected cells, bacteria, tumour cells) and respond in turn by secreting perforin, a pore forming protein, to kill the target cell. Finally, white blood cells also secrete cytokines to recruit other leukocytes to the site of damage/infection/cancer, including cells of the adaptive immune system, which can identify and respond to the presence of specific antigens. For example, T-lymphocytes activate the production of antibodies in B-lymphocytes (which mature into antibody-making factories called plasma cells). The secreted antibodies help macrophages identify and phagocytose the infecting agent. Antibody producing cells are part of the adaptive immune system because they 
help neutralize specific pathogens and the profile of pathogens that are remembered is increased over time, as new threats are encountered.

Hibernators may be able to suppress the adaptive immune system during torpor, but the innate immune system has not been well-studied. To date, components of the innate immune system such as cytokines, prostaglandins, damage-associated molecular pattern molecules (DAMPs) and their respective the pattern recognition receptors (PRRs), have only been studied in selected tissues and in very few species of hibernator. There is evidence that suggests hibernators may have to deal with sources of endogenous tissue damage during a hibernation bout, a key idea that prompted the studies within this thesis. The organs of ground squirrels seem to avoid tissue dysfunction throughout hibernation even though some tissues, including WAT and BAT, show increased levels of oxidative stress markers. The BAT of torpid arctic ground squirrels (Spermophilus parryii) showed an increased ratio of oxidized glutathione to reduced glutathione relative to cold-adapted, euthermic ground squirrels, suggesting increased production of reactive oxygen intermediates during torpor (Orr et al., 2009). Oxidative stress may increase during arousal, since protein carbonyl levels and thiobarbituric acid reactive substances (TBARS, a marker of lipid peroxidation) were increased in BAT from aroused animals relative to torpor. These data, in addition to the discovery of increased levels of a PRR called the receptor of advanced glycation end products (RAGE) in both WAT and BAT of 13-lined ground squirrels (Logan and Storey, 2018) suggested that the adipose tissues of hibernators may experience and respond to endogenous tissue damage during torpor or arousal.

Most molecular studies investigating the protective mechanisms used by hibernators have centered on protein chaperones, antioxidants, and anti-apoptotic proteins (Figure 1.3). Focusing in on white and brown adipose, the tissues of interest in this thesis, there is evidence that a variety of hibernators upregulate protective antioxidants in their adipose depots, to maintain a balance between pro-oxidant and anti-oxidant species as they transition through a torpor bout, with many 
changes in pro-survival and stress-inducible proteins occurring in late torpor and early arousal (Figure 1.3). The WAT and BAT of European ground squirrels (Spermophilus citellus) showed decreased levels of glutathione peroxidase but both fat depots actively upregulated other antioxidant enzymes during torpor including superoxide dismutase 1 and 2 (SOD1, SOD2), or catalase (CAT) (Vucetic et al., 2013). WAT of 13-lined ground squirrels also showed upregulation of several antioxidant proteins during torpor including SOD1, SOD2, and thioredoxin (TRX) but BAT only upregulated TRX (Rouble et al., 2014). Acetylation of SOD2 was also investigated in WAT and BAT, given that deacetylation (and subsequent activation) of SOD2 at K68 is known to increase following oxidative stress. In line with BAT only upregulating TRX, BAT acetylated SOD2 (K68) was increased during early torpor and early arousal and WAT SOD2 was less acetylated during torpor (Rouble and Storey, 2015). Protein chaperones including heat-shock protein 90 (HSP90) alpha was upregulated during arousal in BAT and HSP60 was upregulated upon entrance into torpor in WAT (Rouble et al., 2014). Both chaperones protect against long-term tissue damage by mediating the proper folding of nascent proteins or refolding of unfolded proteins. Finally, 13-lined ground squirrel WAT, like other tissues, upregulated antiapoptotic proteins, likely to balance the ratio of pro-cell death and anti-cell death during torpor (Logan et al., 2016). As an example, pro-apoptotic caspase-3 levels were increased during arousal (relative to torpor) but so were the levels of anti-apoptotic myeloid leukemia cell differentiation protein (Mcl-1) in ground squirrel WAT. The balance between pro- and anti-apoptotic proteins is what is believed to maintain tissue homeostasis under conditions of stress. Interestingly, BAT did not increase the levels of many anti-apoptotic proteins, suggesting that this tissue may rely on other protective responses to cell stress or manage protective responses seasonally, instead (Rouble et al., 2014). 
The projects within this thesis build upon the existing research on the biochemical and physiological responses that support survival in a mammal capable of metabolic rate depression and could serve to regulate inflammatory signaling.

Overall hypothesis: I hypothesize that brown adipose and white adipose tissue, two tissues that have essential functions for hibernator survival in thermogenesis and lipid-storage, regulate inflammatory signaling during torpors and arousal from torpor.

The studies that make up this thesis aimed to uncover how inflammatory signaling may be controlled in a model hibernator at different levels of regulation (e.g. protein profiles, posttranslational modifications of proteins, gene expression, and non-coding RNA regulation of mRNA transcript fate). Specifically, projects focused on the following goals were carried out to characterize hibernator adipose tissue plasticity at various points of the torpor-arousal cycle.

\subsection{Inflammasome}

Objective 1: Determine if ground squirrel adipose tissues sense and respond to changes in cellular homeostasis as they transition into and out of a torpor bout.

Hypothesis 1: I hypothesize that inflammasome complexes will be differentially regulated in ground squirrel adipose tissues, given their roles in sensing and responding to changes in cellular homeostasis.

After discovering that RAGE, a PRR that senses DAMPs, is upregulated during torpor and arousal in the adipose tissues of ground squirrel, it seemed important to determine if other PRRs were also regulated in torpor. RAGE can increase nuclear factor kappa B (NFאB) activity, which is essential for the upregulation of some inflammasome components. The inflammasome is a cytoplasmic wagon wheel-like structure whose purpose is to sense cell stress and respond by activating caspases, with the end goal of cleaving the inactive pro-forms of inflammatory 
signaling molecules such as IL-1 $\beta$ and IL-18 into fully functional cytokines that can be secreted into the extracellular space. Briefly, receptors containing caspase recruitment domains (CARDs) can associate with the CARD domains of caspases but other receptors require a linker protein such as apoptosis-associated speck-like protein containing a CARD (ASC). ASC can bind caspase CARDs and the pyrin domain (PYD) of specific receptor proteins such as nucleotide binding oligomerization domain-leucine rich repeat-containing (LRR) receptor (NLR) and absent in melanoma 2 (AIM2) receptor (ALR) type receptors. Viral, bacterial or fungal infections, and endogenous cell stress prompt the formation of the inflammasome. AIM2, NLRP3 and NLRP1 were the focus for this thesis because they are cytoplasmic receptors capable of sensing endogenous cell stress, unlike other inflammasome receptors that are specifically activated in response to pathogenic insult. AIM2 can sense viral or bacterial DNA/RNA but it can also sense nuclear or mitochondrial DNA in the cytoplasm and damaged DNA (Bae et al., 2019). NLRP3 can sense $\mathrm{K}^{+}$efflux, $\mathrm{Ca}^{2+}$ influx, ROS, uric acid crystals, and markers of lysosomal and mitochondrial stress (Evavold and Kagan, 2018; He et al., 2016). NLRP1 senses toxins, low ATP levels, and inflammation, but it differs from AIM2 and NLRP3 in that it does not need ASC to bind caspase 1 .

Studying the inflammasome provides a unique opportunity to identify whether hibernator tissues can sense and respond to endogenous DAMPs and what kind of DAMPs may be triggering a response. The inflammasome is highly regulated: some receptors must be "primed" before they are activated, such as NLRP3. Cell membrane receptors (e.g. TNFR) sense initial stimuli, whether it be LPS, cytokines (e.g. TNF $\alpha$ ), or other extracellular stress markers. Membrane receptors signal to the cell to increase the production of inflammatory signaling molecules by activating transcription factors such as $\mathrm{NF \kappa B}$. NFкB initiates the gene expression of NLRP3, pro-IL-1 $\beta$ and pro-IL-18. Inflammasome activation also involves the de-ubiquitinylation of NLRP3 protein at Lys-63 by BRCA1-BRCA2-containing complex (BRCC3), since poly- 
ubiquitinylation leads to NLRP3 degradation (He et al., 2016). Then, the NLRP3 inflammasome can be activated by endogenous stress signals such as lysosomal or mitochondrial damage, ROS, particulate matter brought in via endosomes, and changes in $\mathrm{K}^{+}$and $\mathrm{Ca}^{2+}$ levels. This prompts NLRP3 to associate with ASC and pro-caspase 1, which allows caspase 1 to cleave gasdermin D (GSDMD) such that it can form pores in the membrane that allow cleaved IL-1 $\beta$ and IL-18 to exit into the extracellular space. GSDMD pore formation can also result in pyroptosis of the cell, which is a controlled form of necrosis that results in cell swelling and cell rupture, releasing proinflammatory cytokines and DAMPs into the extracellular space (Evavold and Kagan, 2018).

The inflammasome is regulated by many of the same tenets known to regulate whole body metabolism in hibernators: differential transcript expression and translation, reversible posttranslational modifications, and changes in enzyme activity. Thus, hibernators could possibly respond to endogenous damage, to enhance tissue homeostasis, through rapid and reversible molecular mechanisms.

\subsection{Cytokines and tissue remodeling proteins}

Objective 2: Quantify markers of tissue inflammation and tissue remodeling during torpor and arousal in ground squirrel adipose tissues.

Hypothesis 2: I propose that inflammatory cytokines and matrix metalloproteinases will be regulated as a function of the torpor-arousal cycle.

Cytokines are small proteins that are secreted from cells to enact functions on the cell that secreted them (autocrine), neighbouring cells (paracrine) and in distal tissues (endocrine). Interleukins are a class of highly studied cytokine that are produced by and enact functions in leukocytes (white blood cells). However, cytokines are not solely expressed by immune cells, and have been shown to increase in adipocytes following stress (Choe et al., 2016). Pro-inflammatory cytokines including IL-1, IL-18, TNF $\alpha$ and IL-6 are upregulated in the adipose tissues of humans 
suffering from obesity, while anti-inflammatory cytokines like IL-10 are downregulated (Choe et al., 2016; Solinas, 2012). After binding their respective membrane receptors, they may contribute to tissue inflammation and heat production by upregulating the expression and activity of enzymes involved in prostaglandin synthesis (Solinas, 2012). Some anti-inflammatory cytokines like IL-1Ra may also be upregulated in obesity (Solinas, 2012). Interestingly, some hibernator tissues including the lung, intestine and brain have already been shown to differentially regulate cytokines during torpor and upon arousal (Bohr et al., 2014; Cogut et al., 2017; Tessier et al., 2015), emphasizing the need to investigate cytokine production in WAT and BAT, two tissues that are known to have endocrine functions. The measurement of cytokines can provide some information about cell stress but it is also important to look at inhibitors of cytokine signaling. Soluble receptors are inhibitors of cytokine signaling that serve as decoy receptors. They scavenge cytokines to prevent them from docking to receptors that can initiate intracellular signaling cascades.

Some matrix metalloproteinases (MMPs) are activated by cytokine-mediated signaling cascades. For instance, MMP2 and MMP3 are upregulated by some of the transcription factors (e.g. AP-1, AP-2, NFאB and CREB) that are activated by pro-inflammatory cytokines IL-1 and TNF $\alpha$ (Fanjul-Fernández et al., 2010). MMPs are produced as pre-pro-proteins that lose their secretory signal when they are released into the extracellular space. Serine proteases (e.g. plasmin, MT1-MMP) cleave their N-terminal pro-domains to activate them. Once activated, MMPs can help sculpt the extracellular space by cleaving collagen, fibronectin, laminin, and other proteins; steps that are necessary for adipogenesis, angiogenesis, immune cell infiltration, hypertrophy and hyperplasia of adipocytes (Choe et al., 2016; Pellegrinelli et al., 2016; RuizOjeda et al., 2019). MMP2 and MMP3 were of focus in this thesis due to their differential regulation in the adipose tissues of obesity models and their importance in regulating the extracellular matrix (ECM) in response to adipogenesis and adipocyte hypertrophy (Pellegrinelli 
et al., 2016; Ruiz-Ojeda et al., 2019). Interestingly, active MMP2 and MMP3 can cleave the proforms of other MMPs and pro-inflammatory cytokines such as pro-IL-1 $\beta$, pro-TNF $\alpha$, and monocyte-chemotactic protein 3 (MCP3) to regulate their activity. Tissue inhibitor of metalloproteinases (TIMPs) inhibit MMPs by binding to them.

Markers of inflammation and extracellular remodeling are important to measure in torpid and early arousal animals to help understand if activation of the innate immune response and tissue remodeling are necessary for maintaining tissue homeostasis when an animal is metabolically depressed or arousing from torpor.

\subsection{MicroRNA}

Objective 3: Characterize the conserved adipose microRNAs that change expression in response to torpor.

Hypothesis 3: I hypothesize that changes to the miRNAome of both WAT and BAT will be involved in inhibiting pro-inflammatory signaling to prevent chronic inflammation and promote tissue homeostasis during torpor.

Mature microRNAs (miRNAs) are small (18 - $22 \mathrm{nt}$ ), highly-conserved, non-coding RNA molecules that are capable of inhibiting virtually all biological functions via the interactions of complementary miRNA binding to mRNA transcripts. One miRNA can target multiple transcript sequences and a single mRNA can be bound by a variety of miRNAs. Typically, imperfect binding between the mRNA and the seed sequence of the miRNA (nucleotides $\sim 2-8$ ) results in the sequestration of the targeted mRNA for storage and translational repression, whereas perfect complementarity results in the degradation of the transcript by endonucleases (Storey, 2015). What's more, their expression is inducible, rapid, and reversible, making them important regulators of gene expression in animals capable of metabolic rate suppression. MiRNAs are differentially regulated in mammalian torpor, suggesting that miRNAs could facilitate the shut- 
down of energy expensive processes (Storey, 2015). Their expression patterns may also shed light on the pathways that are less inhibited and therefore important for survival when metabolically suppressed. For example, in the pilot study that measured a set of 20 miRNAs in the WAT and BAT of hibernating ground squirrels, many miRNAs involved in regulating MAPK signaling were downregulated, suggesting MAPK kinases may be important in regulating cellular processes during torpor by reversible phosphorylation. By contrast, miRNAs that increased in WAT were found to have protein targets in the TGF- $\beta$ signaling pathway, suggesting this pathway is inhibited during torpor.

MiRNA biogenesis begins with the transcription of pri-miRNA by RNA polymerase II/III. Pri-miRNA are cleaved into 60-70 nucleotide pre-miRNAs by DiGeorge Syndrome Critical Region 8 (DGCR8) and Drosha, a ribonuclease III enzyme. Pre-miRNAs have a characteristic hairpin structure and a 3' overhang of 2 nucleotides long. Exportin-5 is used to shuttle the premiRNAs out of the nucleus and in the cytoplasm, another ribonuclease III enzyme, Dicer, cleaves the terminal loop structure, leaving a short dsRNA molecule. Both strands are loaded onto argonaute proteins of the RNA induced silencing complex (RISC) and the strand that is less stable at its 5' end is the one that is considered the guide strand and is retained by the complex. The passenger strand is removed from the RISC and degraded.

Until the work performed in this thesis, the entire repertoire of ground squirrel miRNAs from WAT and BAT was not explored, and it was largely unknown which metabolic pathway may be targeted by non-coding RNAs in adipose tissue. In this thesis, sequencing data from the Illumina next-generation sequencing platform was analyzed to characterize the expression patterns of conserved miRNAs and determine if they may regulate damage sensing and response pathways.

Finally, it is important to note that throughout this thesis, relative increases or decreases compared to the control value are interpreted as changes in gene expression, whether it be an 
increase in the expression of miRNA, mRNA, or an increase in protein synthesis. It is important to state that I did not measure the synthesis and degradation of a miRNA, mRNA transcript, or protein, nor did I assess their translocation into or out of the cell. Instead, relative gene levels or amounts were measured as an estimation of overall gene expression patterns. For instance, if relative levels of a transcript increased, this could indicate that synthesis of a gene was increased relative to its degradation. 


\subsection{References}

Bae, J.H., Jo, S.I., Kim, S.J., Lee, J.M., Jeong, J.H., Kang, J.S., Cho, N.-J., Kim, S.S., Lee, E.Y., Moon, J.S., 2019. Circulating cell-free mtDNA contributes to AIM2 inflammasome-mediated chronic inflammation in patients with type 2 diabetes. Cells 8, 328. https://doi.org/10.3390/cells8040328

Bohr, M., Brooks, A.R., Kurtz, C.C., 2014. Hibernation induces immune changes in the lung of 13-lined ground squirrels (Ictidomys tridecemlineatus). Dev. Comp. Immunol. 47, 178-184. https://doi.org/10.1016/j.dci.2014.07.011

Bouma, H.R., Strijkstra, A.M., Boerema, A.S., Deelman, L.E., Epema, A.H., Hut, R.A., Kroese, F.G.M., Henning, R.H., 2010. Blood cell dynamics during hibernation in the European ground squirrel. Vet. Immunol. Immunopathol. 136, 319-323. https://doi.org/10.1016/j.vetimm.2010.03.016

Burnett, W.L., 1914. The striped spermophiles or ground squirrels of Colorado (Citellus tridecemlineatus pallidus and Citellus tridecemlineatus parvus).

Cahill, J.E., Lewart, R.M., Jaroslow, B.N., 1967. Effect of hibernation on course of infection and immune response in Citellus tridecemlineatus infected with Nippostrongylus brasiliensis. J. Parasitol. 53, $110-115$.

Cannon, B., Nedergaard, J., 2004. Brown adipose issue: function and physiological significance. Physiol. Rev. 84, 277-359. https://doi.org/10.1152/physrev.00015.2003

Choe, S.S., Huh, J.Y., Hwang, I.J., Kim, J.B.J.I., Kim, J.B.J.I., 2016. Adipose tissue remodeling: its role in energy metabolism and metabolic disorders. Front. Endocrinol. (Lausanne). 7, 30. https://doi.org/10.3389/fendo.2016.00030

Cogut, V., Bruintjes, J.J.J., Eggen, B.J.L.J.L., van der Zee, E.A.A., Henning, R.H.H., 2017. Brain inflammatory cytokines and microglia morphology changes throughout hibernation phases in Syrian hamster. Brain. Behav. Immun. 68, 17-22. https://doi.org/10.1016/j.bbi.2017.10.009

Cooper, S., Sell, S., Nelson, L., Hawes, J., Benrud, J.A., Kohlnhofer, B.M., Burmeister, B.R., Flood, V.H., 2016. Von Willebrand factor is reversibly decreased during torpor in 13-lined ground squirrels. J. Comp. Physiol. B Biochem. Syst. Environ. Physiol. 186, 131-139. https://doi.org/10.1007/s00360015-0941-5

Evavold, C.L., Kagan, J.C., 2018. How inflammasomes inform adaptive immunity. J. Mol. Biol. 430, $217-$ 237. https://doi.org/10.1016/j.jmb.2017.09.019

Fanjul-Fernández, M., Folgueras, A.R., Cabrera, S., López-Otín, C., 2010. Matrix metalloproteinases: Evolution, gene regulation and functional analysis in mouse models. Biochim. Biophys. Acta - Mol. Cell Res. 1803, 3-19. https://doi.org/10.1016/j.bbamcr.2009.07.004

Frank, C.L., Storey, K.B., 1995. The optimal depot fat composition for hibernation by golden-mantled ground squirrels (Spermophilus lateralis). J. Comp. Physiol. B 164, 536-542. https://doi.org/10.1007/BF00261394

Geiser, F., 2004. Metabolic rate and body temperature reduction during hibernation and daily torpor. Annu. Rev. Physiol. 66, 239-74. https://doi.org/10.1146/annurev.physiol.66.032102.115105

Grabek, K.R., Martin, S.L., Hindle, A.G., 2015. Proteomics approaches shed new light on hibernation physiology. J. Comp. Physiol. B Biochem. Syst. Environ. Physiol. 185, 607-627. https://doi.org/10.1007/s00360-015-0905-9

He, Y., Hara, H., Núñez, G., 2016. Mechanism and regulation of NLRP3 inflammasome activation. Trends Biochem. Sci. 41, 1012-1021. https://doi.org/10.1016/j.tibs.2016.09.002

Helgen, K.M., Cole, F.R., Helgen, L.E., Wilson, D.E., Helgen, K.M., Cole, F.R., Helgen, L.E., Wilson, D.O.N.E., 2009. Generic revision in the holarctic ground squirrel Genus Spermophilus. J. Mammal. 90, 270-305. 
Hisaw, F.L., Emery, F.E., 1927. Food selection of ground squirrels, Citellus tridecemlineatus. J. Mammal. 8, 41. https://doi.org/10.2307/1373396

Hittel, D., Storey, K.B., 2001. Differential expression of adipose- and heart-type fatty acid binding proteins in hibernating ground squirrels. Biochim. Biophys. Acta 1522, 238-243. https://doi.org/10.1016/S0167-4781(01)00338-4

Kisser, B., Goodwin, H.T., 2012. Hibernation and overwinter body temperatures in free-ranging 13-lined ground squirrels, Ictidomys tridecemlineatus. Am. Midl. Nat. 167, 396-409. https://doi.org/10.1674/0003-0031-167.2.396

Landau, B.R., Dawe, A.R., 1958. Respiration in the hibernation of the 13-lined ground squirrel. Am. J. Physiol. Content 194, 75-82. https://doi.org/10.1152/ajplegacy.1958.194.1.75

Logan, S.M., Luu, B.E., Storey, K.B., 2016. Turn down genes for WAT? Activation of anti-apoptosis pathways protects white adipose tissue in metabolically depressed 13-lined ground squirrels. Mol. Cell. Biochem. 416, 47-62. https://doi.org/10.1007/s11010-016-2695-0

Logan, S.M., Storey, K.B., 2018. Pro-inflammatory AGE-RAGE signaling is activated during arousal from hibernation in ground squirrel adipose. PeerJ 6, e4911. https://doi.org/10.7717/peerj.4911

MacCannell, A.D. V., Sinclair, K.J., McKenzie, C.A., Staples, J.F., 2018. Environmental temperature effects on adipose tissue growth in a hibernator. J. Exp. Biol. jeb.194548. https://doi.org/10.1242/jeb.194548

McCarley, H., 1966. Annual cycle, population dynamics and adaptive behavior of Citellus tridecemlineatus. J. Mammal. 47, 294-316. https://doi.org/10.2307/1378126

Merriman, D.K., Lahvis, G., Jooss, M., Gesicki, J.A., Schill, K., 2012. Current practices in a captive breeding colony of 13-lined ground squirrels (Ictidomys tridecemlineatus). Lab Anim. (NY). 41, 315-325. https://doi.org/10.1038/laban.150

Nedergaard, J., Cannon, B., 1984. Preferential utilization of brown adipose tissue lipids during arousal from hibernation in hamsters. Am. J. Physiol. 247. https://doi.org/10.1152/ajpregu.1984.247.3.r506

Orr, A.L., Lohse, L.A., Drew, K.L., Hermes-Lima, M., 2009. Physiological oxidative stress after arousal from hibernation in Arctic ground squirrel. Comp. Biochem. Physiol. 153, 213-221. https://doi.org/10.1016/j.cbpa.2009.02.016.Physiological

Pellegrinelli, V., Carobbio, S., Vidal-Puig, A., 2016. Adipose tissue plasticity: how fat depots respond differently to pathophysiological cues. Diabetologia 59, 1075-1088. https://doi.org/10.1007/s00125016-3933-4

Rouble, A.N., Hefler, J., Mamady, H., Storey, K.B., Tessier, S.N., 2013. Anti-apoptotic signaling as a cytoprotective mechanism in mammalian hibernation. PeerJ 1, e29. https://doi.org/10.7717/peerj.29

Rouble, A.N., Storey, K.B., 2015. Characterization of the SIRT family of NAD ${ }^{+}$-dependent protein deacetylases in the context of a mammalian model of hibernation, the 13-lined ground squirrel. Cryobiology 71, 334-343. https://doi.org/10.1016/j.cryobiol.2015.08.009

Rouble, A.N., Tessier, S.N., Storey, K.B., 2014. Characterization of adipocyte stress response pathways during hibernation in 13-lined ground squirrels. Mol. Cell. Biochem. 393, 271-282. https://doi.org/10.1007/s11010-014-2070-y

Ruf, T., Geiser, F., 2015. Daily torpor and hibernation in birds and mammals. Biol Rev Camb Philos Soc. 90, 891-926.

Ruiz-Ojeda, F.J., Méndez-Gutiérrez, A., Aguilera, C.M., Plaza-Díaz, J., 2019. Extracellular matrix remodeling of adipose tissue in obesity and metabolic diseases. Int. J. Mol. Sci. 20. https://doi.org/10.3390/ijms20194888 
Solinas, G., 2012. Molecular pathways linking metabolic inflammation and thermogenesis. Obes. Rev. 13, 69-82. https://doi.org/10.1111/j.1467-789X.2012.01047.x

Storey, K.B., 2015. Regulation of hypometabolism: insights into epigenetic controls. J. Exp. Biol. 218, 150-159. https://doi.org/10.1242/jeb.106369

Storey, K.B., Heldmaier, G., Rider, M.H., 2010. Mammalian hibernation: physiology, cell signaling, and gene control on metabolic rate depression, in: Lubzens, E. (Ed.), Dormancy and Resistance in Harsh Environments. Springer-Verlag Berlin Heidelberg, pp. 227-252. https://doi.org/10.1007/978-3-642$12422-8$

Storey, K.B., Storey, J.M., 2005. Mammalian hibernation: biochemical adaptation and gene expression, in: Storey, K.B. (Ed.), Functional Metabolism: Regulation and Adaptation. John Wiley \& Sons, Inc., pp. 443-471. https://doi.org/10.1002/047167558X.ch16

Tessier, S.N., Katzenback, B. a., Pifferi, F., Perret, M., Storey, K.B., 2015. Cytokine and antioxidant regulation in the intestine of the gray mouse lemur (Microcebus murinus) during torpor. Genomics, Proteomics Bioinforma. 13, 127-135. https://doi.org/10.1016/j.gpb.2015.03.005

Van Vuren, D.H., Ordeñana, M.A., 2012. Factors influencing burrow length and depth of ground-dwelling squirrels. J. Mammal. 93, 1240-1246. https://doi.org/10.1644/12-MAMM-A-049.1

Vaughan, D.K., Gruber, A.R., Michalski, M.L., Seidling, J., Schlink, S., 2006. Capture, care, and captive breeding of 13-lined ground squirrels, Spermophilus tridecemlineatus. Lab Anim. (NY). 35, 33-40. https://doi.org/10.1038/laban0406-33

Vucetic, M., Stancic, A., Otasevic, V., Jankovic, A., Korac, A., Markelic, M., Velickovic, K., Golic, I., Buzadzic, B., Storey, K.B., Korac, B., 2013. The impact of cold acclimation and hibernation on antioxidant defenses in the ground squirrel (Spermophilus citellus): An update. Free Radic. Biol. Med. 65, 916-924. https://doi.org/10.1016/j.freeradbiomed.2013.08.188

Withers, P.C., Cooper, C.E., 2010. Metabolic depression: a historical perspective. pp. 1-23. https://doi.org/10.1007/978-3-642-02421-4_1

Yan, J., Barnes, B.M., Kohl, F., Marr, T.G., 2008. Modulation of gene expression in hibernating arctic ground squirrels. Physiol. Genomics 32, 170-181. https://doi.org/10.1152/physiolgenomics.00075.2007 


\subsection{Figures}

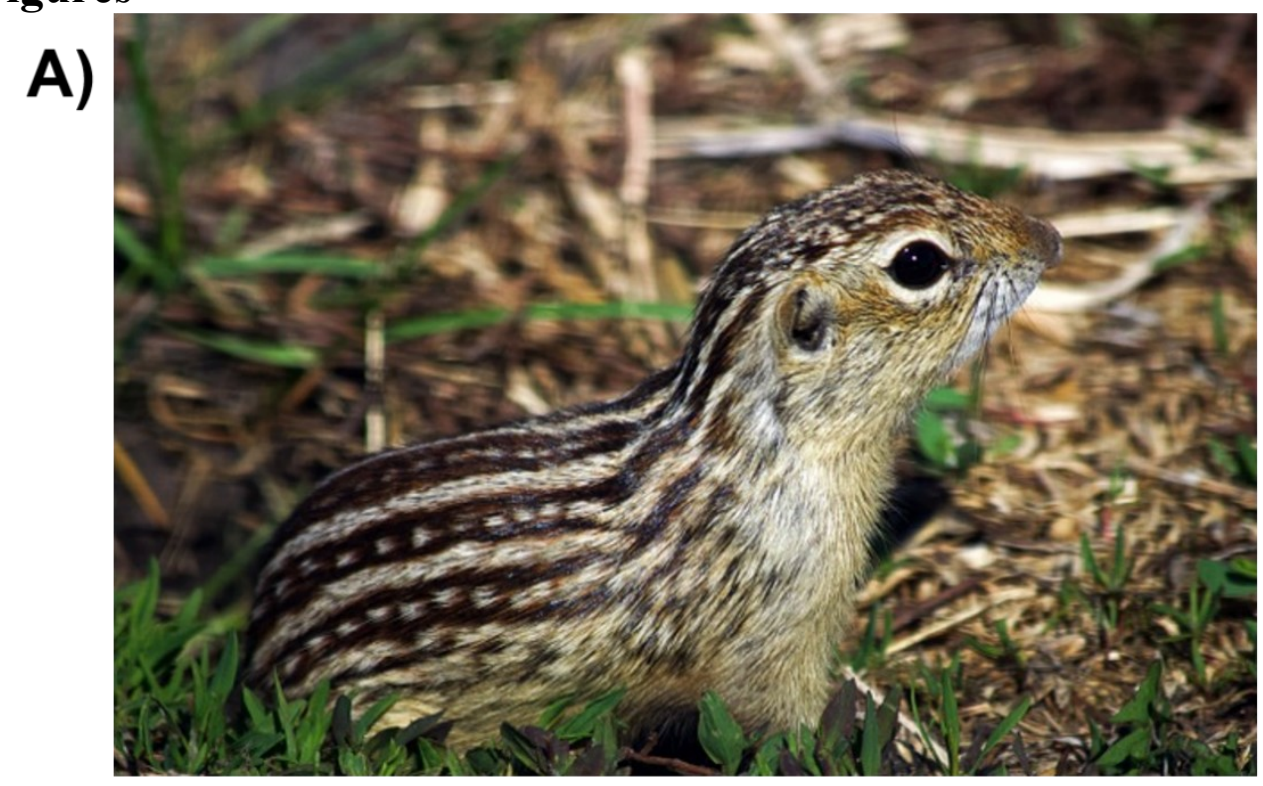

\section{B)}

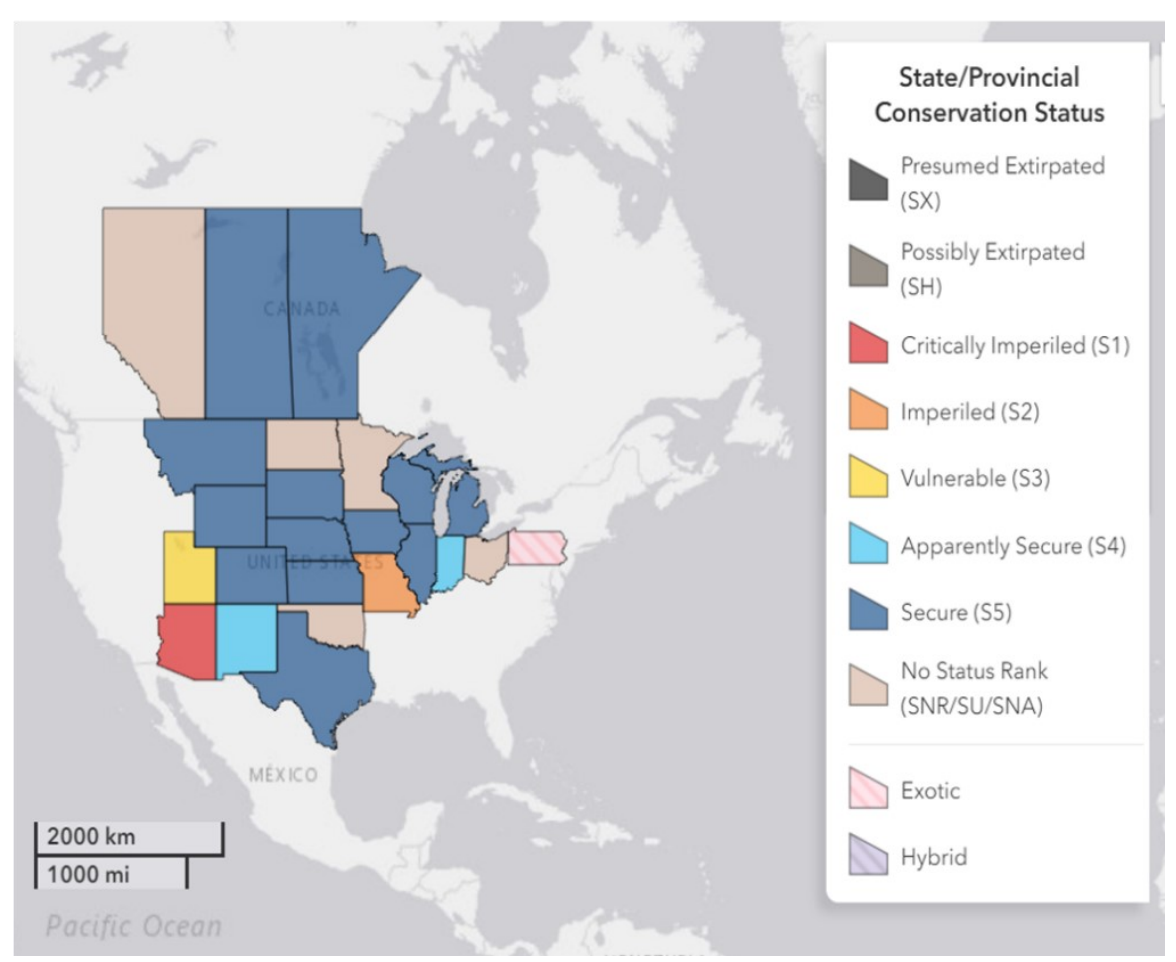

Figure 1.1: 13-lined ground squirrels inhabit the grasslands, farmlands, and golf courses of North America. A) Image of Ictidomys tridecemlineatus courtesy of Dr. Ryan Sprenger, and national and state/province distribution of I. tridecemlineatus from explorer.natureserve.org. 

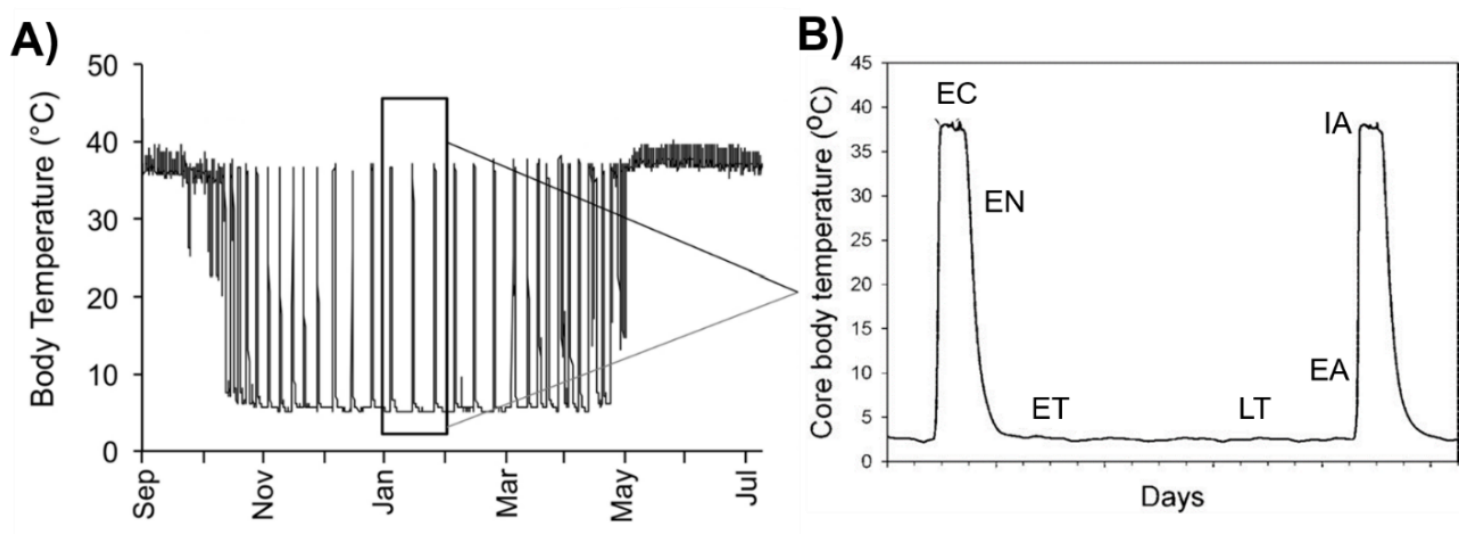

Figure 1.2: Torpor-arousal cycle of a hibernating ground squirrel, showing shallow torpor bouts are used at the beginning of the hibernation season, and are followed by deeper and longer (approximately 2 weeks long) torpor-bouts. Euthermic in the cold room control (EC) ground squirrels are sampled at euthermia $\left(37^{\circ} \mathrm{C}\right)$. Ground squirrels entering torpor $(\mathrm{EN})$ have decreasing body temperatures between $18-31^{\circ} \mathrm{C}$. Early torpid (ET) and late torpid (LT) ground squirrels have body temperatures between $5-8{ }^{\circ} \mathrm{C}$ but differ in terms of the number of days they have spent in torpor before euthanasia ( $<1$ day vs at least 5 days, respectively). Early arousal (EA) ground squirrels have increasing body temperatures between $9-12{ }^{\circ} \mathrm{C}$ and interbout aroused ground squirrels are euthermic for less than 18 hours. Graphs are modified from (Grabek et al., 2015; Yan et al., 2008). 


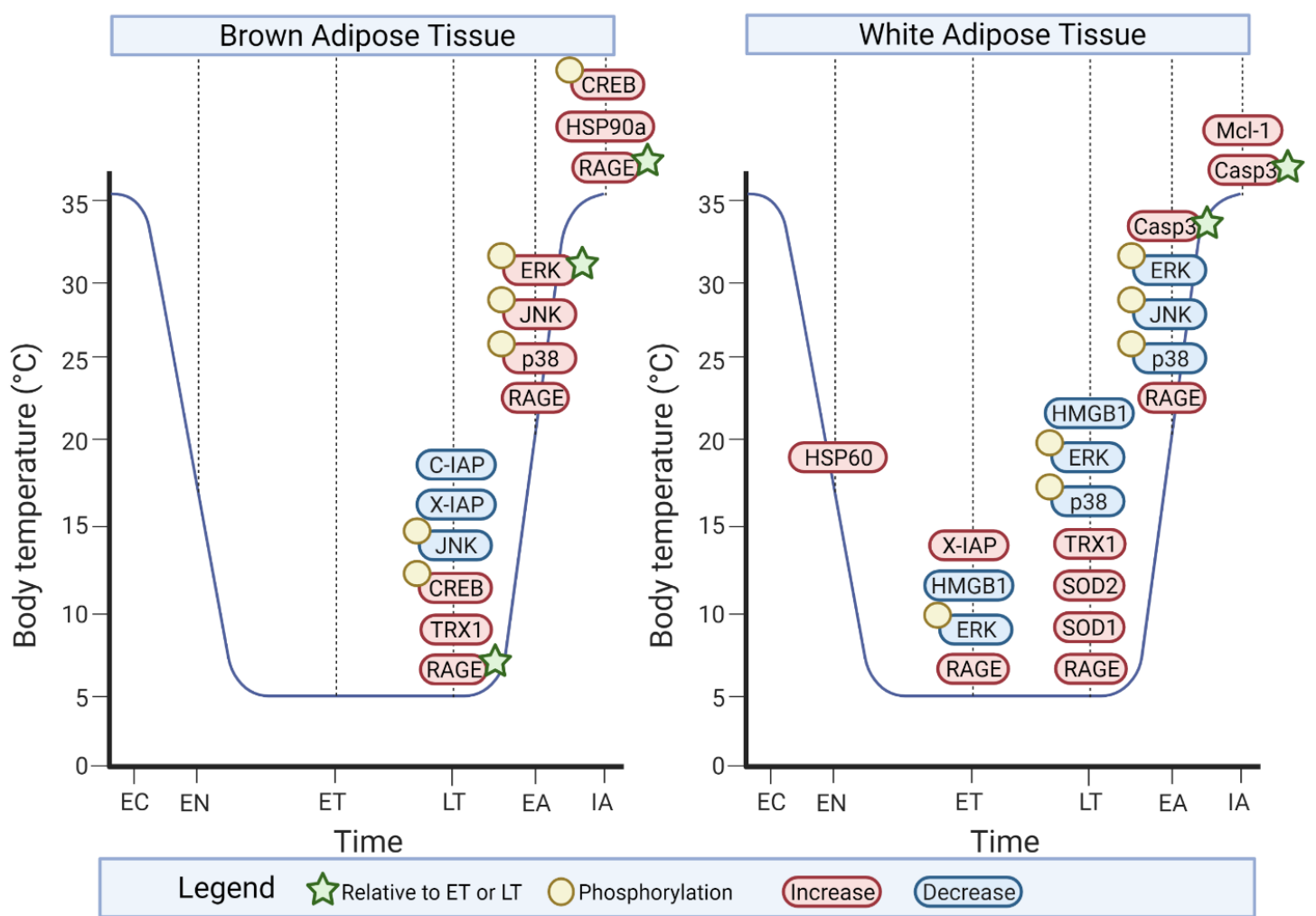

Figure 1.3: Summary of the expression patterns of some antioxidants (thioredoxin, TRX; superoxide dismutase 1 and 2, SOD1, SOD2), protein folding chaperones (heat shock proteins HSP60, HSP90a), apoptosis (cell death and x-linked inhibitor of apoptosis proteins C-IAP and XIAP; myeloid leukemia cell differentiation protein, Mcl-1; caspase-3, Casp3), damage-associated molecular pattern molecule (DAMP) signaling (High mobility group box 1, HMGB1; receptor for advanced glycation end products, RAGE), and stress inducible signaling proteins (cAMP response element-binding protein, CREB; c-Jun N-terminal kinase, JNK, p38, extracellular signal-regulated kinase, ERK) in the brown adipose and white adipose tissues. In general, WAT increases the expression of antioxidant proteins and anti-apoptotic proteins more than BAT, which increases stress-inducible protein kinase signaling pathways more than WAT. Created using BioRender. 
2 Inflammasome signaling could be used to sense and respond to endogenous damage in brown but not white adipose tissue of a hibernating ground squirrel 


\subsection{Abstract}

Small mammalian hibernators use metabolic suppression to enhance survival during the winter. Torpor is punctuated by periods of euthermia used to clear metabolic by-products and damaged cell components. The current study was performed to determine if the innate immune system, specifically NLRP and AIM2 inflammasome signaling, may detect and respond to cell stress during hibernation. Nlrp3, Casp 1 , and $I l 1 b$ genes were significantly upregulated in brown adipose tissue (BAT) during arousal with respect to the euthermic control, suggesting increased NLRP3 inflammasome priming. NLRP3, IL-18, and gasdermin D protein levels increased during torpor, indicating a lag between inflammasome priming and formation. AIM2 and gasdermin D levels increased in BAT during arousal, as did caspase-1 activity. Thus, non-shivering thermogenesis may generate pro-inflammatory triggers of inflammasome signaling. This study is the first to support a role for inflammasome signaling in sensing cellular perturbations at various points of the torpor-arousal cycle, in metabolically-active BAT, but not white adipose tissue (WAT).

Keywords: Ictidomys tridecemlineatus, NLRP3, AIM2, caspase-1, innate immunity, torporarousal cycle 


\subsection{Introduction}

Hibernation is a strategy that some mammals use to avoid the metabolic costs associated with maintaining euthermia in winter environmental conditions. Torpor in a typical hibernating ground squirrel is characterized by a decrease in metabolism by $95-99 \%$ of the basal metabolic rate and a decline in body temperature from $37^{\circ} \mathrm{C}$ to $5-10{ }^{\circ} \mathrm{C}$, although some species of ground squirrel can reduce their body temperature down to $-2.9{ }^{\circ} \mathrm{C}$ (Storey, 2010; Wang and Lee, 1996). Hibernating ground squirrels cycle between periods of sustained torpor, which can extend up to two weeks, and brief episodes of euthermia called interbout arousals that last up to 48 hours (Wang and Lee, 1996). Interbout arousals may be important for hibernators to sleep and/or contend with toxic accumulation of metabolic by-products and damaged macromolecules, as these animals typically arouse in the spring with little to no organ damage (Cogut et al., 2017; Wickler et al., 1991). Previous studies have confirmed that damage markers related to inflammation and oxidative stress increase in some hibernator tissues during a torpor-bout but few investigations have been completed regarding the response to these stress signals (Carey et al., 2000; Frank and Storey, 1995; Orr et al., 2009). As such, the goal of the current study was to investigate if, when, and how hibernators may sense cellular damage (e.g. protein adducts, DNA damage, or lipid peroxidation) and if innate immunity pathways may be upregulated to mediate unfavourable damage marker accumulation in advance of or during interbout arousals.

White adipose tissue (WAT) stores the hibernator's primary source of energy in the form of triglycerides and brown adipose tissue (BAT) is rich in mitochondria, used to maintain the thermogenic set-point and rapidly increase body temperature through non-shivering thermogenesis (NST) (Ballinger et al., 2016; Boyer et al., 1998; Wang et al., 1997). Further, both adipose depots are regulators of whole-body homeostasis through their secretion of hormones and cytokines (Zhu et al., 2019). Their roles in fat storage, thermogenesis, and the production and release of adipokines make these fat depots prime locations for cell stress. For instance, 
adipocytes in hibernator WAT must hypertrophy to accommodate the rapid increase of lipids in the fall and thereafter remain expanded throughout the hibernation season (Young et al., 1982). Furthermore, during torpor, ground squirrels may only breathe a few breaths every hour and the oxygen pumped in viscous blood may be rationed to the most metabolically active tissues (Harker and Webb, 1987; Webb and Milsom, 2017). Thus, hypertrophied adipocytes may experience cell membrane stress and if not properly vascularized, low-oxygen stress may encourage the formation of reactive oxygen species (ROS). Indeed, chronic diet-induced adiposity exacerbates low-oxygen stress and inflammatory signaling in non-hibernating models (Badran et al., 2014; Olea et al., 2014). ROS is also a by-product of BAT-mediated NST. To increase body temperature, ATP synthesis is uncoupled from the electron transport chain in mitochondria in BAT. As a hibernator arouses from torpor, increases in breathing rate and cellular respiration can result in an influx of ROS and other endogenous cellular stress. Recently, we discovered an increase in the protein levels of the receptor for advanced glycation end products (RAGE) during torpor and arousal in the white and brown adipose tissues of 13-lined ground squirrels (Logan and Storey, 2018), which suggests that hibernators may have specific mechanisms to sense accumulating damage markers such as glycated proteins and markers of cell membrane disruption. Other studies indicate that markers of oxidative stress are present in higher levels in the frontal cortex and brown adipose tissues of euthermic or interbout aroused Arctic ground squirrel compared to hibernating Arctic ground squirrels (Orr et al., 2009).

The inflammasome is a large complex that forms in response to the sensing of cellular damage and is used to communicate to surrounding cells that inflammation should ensue. First, a stress signal such as mitochondrial ROS "primes" inflammasome signaling by inducing nuclear factor kappa-light-chain-enhancer of activated B cells (NFKB)-mediated expression of prointerleukin-1 $\beta$ /pro-interleukin-18 cytokines and pattern recognition receptors (PRRs) such as Nucleotide-binding oligomerization domain, Leucine rich Repeat and Pyrin domain 3 (NLRP3) 
(Alfonso-Loeches et al., 2016; Bauernfeind et al., 2009). In the second step of inflammasome activation, another stress signal stimulates the association of the pyrin domain (PYD) of the inflammasome receptor (i.e. NLRP3 or absent in melanoma 2 (AIM2)) with the PYD domain of ASC, more formally known as apoptosis-associated speck-like protein containing a caspase recruitment domain (CARD). The gene that encodes the ASC protein is called Pycard, because the PYD and CARD domains are so integral to its function. However, PRRs such as Nucleotidebinding oligomerization domain, Leucine rich Repeat and Pyrin domain 1 (NLRP1) and NLR Family CARD Domain Containing 4 (NLRC4) do not need to associate with ASC because they can bind to pro-caspase-1 directly. NLRP3 activation (and subsequent ASC association) is stimulated by a range of stimuli such as $\mathrm{K}^{+}$efflux, $\mathrm{Ca}^{2+}$ influx, $\mathrm{ROS}$, uric acid crystals, lysosomal rupture, and markers of mitochondrial dysfunction such as mtDNA, mtROS and cardiolipin, all of which can appear as the result of endogenous cell stress and damage (Afonina et al., 2017; Evavold and Kagan, 2018; He et al., 2016). The inflammasome receptor AIM2 can also detect endogenous cellular damage and is specifically stimulated by DNA, including mtDNA, nuclear DNA in the cytoplasm, though this receptor also detects exogenous bacterial or viral DNA (Bae et al., 2019). Unlike AIM2 and NLRP3 inflammasomes, NLRP1 inflammasomes can form in the absence of ASC, typically in response to toxins, but they may be able to form in response to endogenous cell stress (e.g. autoinflammatory disease and low levels of ATP) (Mitchell et al., 2019). Once PRRs are associated with ASC, if required, the CARD domains of ASC and procaspase-1 dimerize, and the associated inflammasome proteins may further arrange into a wagon wheel-like multi-subunit complex. After assembly, inflammasomes catalyze the cleavage of proinflammatory pro-caspase 1 in canonical inflammasome signaling, or pro-caspase 11 (mouse homologue of human caspases 4 and 5) in non-canonical signaling, which leads to the cleavage and activation of cytokines IL-1 $\beta$ and IL-18. IL-1 $\beta$ and IL-18 can then be secreted from the cell through pores made of cleaved gasdermin D. Upon entering the intracellular space, active IL-18 
and IL- $1 \beta$ bind to IL receptors to activate inflammatory signaling or recruit more immune cells to the site of damage (He et al., 2016).

Since inflammasome priming and formation only occurs if a stress is sensed, it was postulated that hibernator adipose tissues may invoke inflammasome formation to regulate inflammation and reduce cellular damage. It was anticipated that BAT would show more changes in inflammasome activation than WAT over the course of a torpor-arousal cycle, due to the role of BAT in NST, and the more passive role of WAT in fat storage and mobilization. To investigate the activation of inflammasomes (NLRP1, NLRP3, and AIM2) over the course of a torpor bout in a model hibernator, the 13-lined ground squirrel (Ictidomys tridecemlineatus), relative transcript expression was used to investigate priming events, relative total protein levels helped determine which inflammasome may be activated in each tissue, and a caspase-1 activity assay was used to quantify inflammasome activation.

\subsection{Methods}

\subsubsection{Animals}

Male ground squirrels (Ictidomys tridecemlineatus) weighing 150-300 g were wild captured by a United States Department of Agriculture licensed trapper (TLS Research, Bloomingdale, IL) and transported to the laboratory of Dr. J.M. Hallenback at the Animal Hibernation Facility, National Institute of Neurological Disorders and Stroke (NINDS) (NIH, Bethesda, MD) where the hibernation experiments were conducted. Animal housing and experimental procedures were approved by the NINDS animal care and use committee (ACUC, \#ASP 1223-05). Each 13-lined ground squirrel was fitted with a sensor chip (IPTT-300; Bio Medic Data Systems) injected subcutaneously while anesthetized with 5\% isofluorane and was housed individually in a shoebox cage at $21^{\circ} \mathrm{C}$. Animals were fed a standard rodent diet and water ad libitum until they gained sufficient lipid stores to enter hibernation. To enable a natural 
transition into torpor, animals were transferred to an environmental chamber at $5{ }^{\circ} \mathrm{C}$ in constant darkness. Body temperature $\left(\mathrm{T}_{\mathrm{b}}\right)$, time, and respiration rate were observed to determine sampling points. All 13-lined ground squirrels had been through torpor-arousal bouts prior to euthanasia by decapitation and tissue sampling, as previously described (McMullen and Hallenbeck, 2010).

Tissue samples were shipped to Carleton University on dry ice and then stored at $-80{ }^{\circ} \mathrm{C}$ until use.

EC designates euthermic, cold room; these euthermic animals had a stable $\mathrm{T}_{\mathrm{b}}\left(37^{\circ} \mathrm{C}\right)$ in the $5{ }^{\circ} \mathrm{C}$ cold room, were able to enter torpor but had not done so for at least 3 days. EN designates entrance; animals were in the entrance phase of the hibernation bout characterized by decreasing $\mathrm{T}_{\mathrm{b}}$ and were sampled when $\mathrm{T}_{\mathrm{b}}$ was $18-31^{\circ} \mathrm{C}$. ET designates early torpor; animals had a stable $\mathrm{T}_{\mathrm{b}}$ of $5-8{ }^{\circ} \mathrm{C}$ for 1 day. $\mathrm{LT}$ designates late torpor; animals that were continuously in deep torpor for at least 5 days with $\mathrm{T}_{\mathrm{b}}$ values of $5-8{ }^{\circ} \mathrm{C}$. EA designates early arousal animals characterized by an increased respiratory rate of $>60$ breaths $/$ min and a rising $\mathrm{T}_{\mathrm{b}}$ of $9-12{ }^{\circ} \mathrm{C}$. IA designates interbout arousal after a multi-day torpor with core body temperature returned to $37^{\circ} \mathrm{C}$ for approximately $18 \mathrm{~h}$.

\subsubsection{RNA extraction and RT-qPCR}

RNA extractions were performed using the TRIzol (Invitrogen, \#15596026) method as directed by the manufacturer and as previously described (Logan et al., 2016). Briefly, approximately $100 \mathrm{mg}$ of BAT and WAT from EC, EN, ET, LT, EA, and IA animals $(\mathrm{n}=4$; except $\mathrm{n}=3$ WAT biological replicates were prepared for EN and EA due to tissue availability of these time points) was weighed and homogenized in $1 \mathrm{~mL}$ of TRIzol using a Polytron PT1200 homogenizer. RNA purity was assessed using the ratio of absorbances 260/280 nm and RNA integrity was tested by visualizing $18 \mathrm{~S}$ and $26 \mathrm{~S}$ ribosomal RNA bands on a $1 \%$ agarose gel with SybrGreen staining. RNA samples were standardized in a total volume of $10 \mu \mathrm{L}$ diethyl pyrocarbonate (DEPC)-autoclaved $\mathrm{ddH}_{2} \mathrm{O}$ such that each biological replicate contained $4 \mu \mathrm{g}$ (brown adipose tissue) or $1.14 \mu \mathrm{g}$ (white adipose tissue) of RNA. The standardized RNA samples 
were incubated with $1 \mu \mathrm{L}$ of Oligo-dT (200 ng/ $\mu \mathrm{L}$ 5'-TTTTTTTTTTTTTTTTTTTTTTV-3'; where $\mathrm{V}=\mathrm{A}, \mathrm{G}$, or $\mathrm{C}$; Sigma Genosys) and placed in a thermocycler at $65^{\circ} \mathrm{C}$ for $5 \mathrm{~min}$, then chilled on ice for $5 \mathrm{~min}$. Reverse transcription was performed with $4 \mu \mathrm{L}$ of $5 \mathrm{X}$ first-strand buffer (Invitrogen, \#8057018), $2 \mu \mathrm{L}$ of 0.1 M DTT (Invitrogen, \#D1532), $1 \mu \mathrm{L}$ of $10 \mathrm{mM}$ dNTPs (\#NUC001; BioShop, Burlington, Canada), and $1 \mu \mathrm{L}$ of MMLV Reverse transcriptase (Invitrogen, \#28025013). Following a 45 min incubation in the thermocycler at $42{ }^{\circ} \mathrm{C}$, RT-qPCR was performed as described previously (Pellissier et al., 2006), using a BioRad CFX Connect apparatus. Forward and reverse primers used for RT-qPCR were designed using National Center for Biotechnology Information (NCBI) sourced I. tridecemlineatus mRNA sequences and the NCBI Primer-BLAST tool. The primers are as follows: Nlrp3 forward 5'GTCCTCCGTGCTCAGAACAA-3' and reverse 5'-CCTCACAGAGGAGCTTGACG-3'; Aim2 forward 5'-TGTTGGCACAGTGGTGTTCT-3' and reverse 5'-GGTGTTTCCCCAGCTTTCCT3'; Pycard forward 5'-TTGTGGACCAACACCGAACA-3' and reverse 5'-

CAGGACCTTGCCATACAGGG-3'; Gsdmd forward 5'-CTGAGCAGGAAGTCCCCAAG-3'; Caspl forward 5'-TGCTGAACACCTGGAACTGT-3' and reverse 5'ACCACACCACCCCTTGATTC-3'; Ill b forward 5'-GTACATCAGCACCTCCCAGG-3' and reverse 5'-TGAGGCTCCTTAGGACACGA-3'; Il18 forward 5'AGGAGTTTTGCTCTCCCGTG-3' and reverse 5'-TCCTAAGACCGTGGAGACTGT-3'; Tbp forward 5'-AGAGTGTGCTGGGAATGCTC-3' and reverse 5'CAGGCTGCTGTTCTGATCCA-3'; Actg forward 5'-CTGTCTTCCCCTCCATCGTG-3' and reverse 5'-CCTCATCCCCAACGTAGCTG-3'; Acta forward 5'AGAACAGCAGGTGTAGTCACG-3' and reverse 5'-AGCCATTGTCACACACGAGG-3'. Tbp and Actg were used as the reference genes for BAT and Acta was used as the reference gene for WAT because of their highly consistent levels across all 6 points of the torpor-arousal cycle. Melt curve analyses was performed to ensure the presence of a single melt peak for each primer set and a dilution series of the template cDNA ensured the single amplicon was not a primer-dimer. To 
confirm the identity of the amplified PCR products, several of the PCR products were sequenced (BioBasic, Markham, ON) and BLASTn (NCBI) was used to determine if the sequence of the product matched the intended sequence.

\subsubsection{Total protein extraction}

Frozen BAT and WAT samples were weighed (70 mg of 4 biological replicates from EC, EN, ET, LT, EA and IA) and crushed into small pieces under liquid nitrogen. Five to seven piston strokes of a glass Dounce pestle were used to homogenize the tissues in ice-cold Cell Signaling Lysis Buffer (EMD Millipore, \#43-040), in a ratio of 1:3 w:v ratio for BAT and a 1:2 ratio for WAT and supplemented with $1 \mathrm{mM}$ sodium orthovanadate, $10 \mathrm{mM}$ sodium fluoride, $10 \mathrm{mM} \beta$ glycerophosphate, $10 \mu \mathrm{L} / \mathrm{mL}$ Protease Inhibitor cocktail (BioShop, \#PIC001.1), and a few crystals of PMSF. Each sample was placed on ice and gently vortexed every $10 \mathrm{~min}$ for $30 \mathrm{~min}$ before centrifugation at $13,500 \times \mathrm{g}$ for $20 \mathrm{~min}$ at $4{ }^{\circ} \mathrm{C}$. The supernatant containing soluble proteins was collected for each sample and total protein concentration was determined for immunoblotting and ELISA samples using the Bio-Rad method (Bio-Rad, \#500-0005). Appropriate amounts of homogenization buffer were added to the samples intended for immunoblotting such that they were all standardized to $10 \mu \mathrm{g} / \mu \mathrm{L}$. Then, $2 \mathrm{X}$ sodium dodecyl sulfate (SDS) loading buffer (100 $\mathrm{mM}$ Tris-base adjusted to $\mathrm{pH} 6.8,4 \% \mathrm{w}: \mathrm{v}$ SDS, $20 \% \mathrm{v}: \mathrm{v}$ glycerol, $0.2 \% \mathrm{w}: \mathrm{v}$ bromophenol blue, $10 \% \mathrm{v}: \mathrm{v} 2$-mercaptoethanol) was added to each sample in a 1:1 v:v ratio and the samples were mixed and boiled. The final $5 \mu \mathrm{g} / \mu \mathrm{L}$ protein samples were stored at $-40{ }^{\circ} \mathrm{C}$ until use.

\subsubsection{Immunoblotting}

Equal amounts $(25 \mu \mathrm{g})$ of prepared total protein extract and $4-5 \mu \mathrm{L}$ of $10.5-175 \mathrm{kDa}$ PiNK Plus pre-stained protein ladder (FroggaBio, \#PM005-0500) were loaded onto 10-15\% SDS-PAGE gels, electrophoresed for 80-100 min at $180 \mathrm{~V}$ using the BioRad Mini Protean III system in Tris-glycine running buffer (0.25 M Tris-base $\mathrm{pH}$ 8.0, 2.45 M glycine, 0.035 M SDS). Proteins were wet-transferred onto polyvinylidene fluoride (PVDF), either at $160 \mathrm{~mA}$ for 90-120 
min (for proteins with molecular weights of $40 \mathrm{kDa}$ or higher) or at $40 \mathrm{~V}$ for $45 \mathrm{~min}$ (for proteins with molecular weights of $40 \mathrm{kDa}$ or lower) in Tris-glycine transfer buffer ( $25 \mathrm{mM}$ Tris $\mathrm{pH} 8.5$, $192 \mathrm{mM}$ glycine and 10\% v:v methanol). Blots were blocked with $2.5-10.0 \% \mathrm{w}: \mathrm{v}$ milk in Trisbuffered saline with Tween-20 (TBST; $50 \mathrm{mM}$ Tris-HCl, $150 \mathrm{mM} \mathrm{NaCl}, 0.05 \%$ v:v Tween-20, $\mathrm{pH}$ 6.8) for $15-30 \mathrm{~min}$. The blots were incubated overnight at $4{ }^{\circ} \mathrm{C}$ on a rocker with primary antibodies diluted 1:500-1:1000 v:v in TBST. Santa Cruz Biotechnology supplied the mouse monoclonal antibodies including those targeting NLRP1 (\#sc-390133), NLRP3 (\#sc-134306), AIM2 (\#sc-293174), Caspase 1 p20 (cleaved) (sc-398715), Caspase 5 p20 (cleaved) (\#sc393346), Gasdermin D (\#sc-81868) and IL-1ß (\#sc-515598). The rabbit monoclonal antibody targeting IL-18 was purchased from Abcam (\#ab207324). Following primary antibody removal, the membranes were incubated for 15-30 min with a horseradish peroxidase (HRP)-linked mouse IgG secondary antibody (Bioshop \#APA005P.2) or an HRP-linked rabbit IgG secondary antibody (Bioshop, \#APA007P.2), diluted 1:4000-1:8000 v:v with TBST. Enhanced chemiluminescence (ECL) reagents were used to visualize the protein bands.

\subsubsection{Caspase-1 activity assay}

A Caspase-1/interleukin-1 converting enzyme (ICE) Colorimetric Assay (BioVision, \#K111) was performed as per the manufacturer's instructions. First, frozen BAT and WAT weighing 20-40 mg were collected from $\mathrm{n}=5$ individual animals under liquid nitrogen. Frozen tissue pieces were homogenized in $50 \mu \mathrm{L}$ of the provided Cell Lysis Buffer with 8-10 strokes of a glass Dounce homogenizer. Protein concentration of each sample was determined using the BioRad method (Bio-Rad, \#500-0005). Active recombinant Human caspase-1 (BioVision, \#1081-25) served as the positive control, as per the recommendation of the Caspase-1/ICE colorimetric assay manufacturer, and was used to generate a standard curve (Appendix B). To determine caspase-1 activity between EC, LT, and EA ground squirrels, $75 \mu \mathrm{g}$ of total BAT or WAT protein sample were suspended in $50 \mu \mathrm{L}$ of Cell Lysis Buffer before adding $55 \mu \mathrm{L}$ of a master mix 
containing $10 \mathrm{mM}$ DTT, $2 \mathrm{x}$ reaction buffer and $5 \mu \mathrm{L} /$ reaction of the caspase substrate $(4 \mathrm{mM}$ YVAD-pNA substrate for a final concentration of $200 \mu \mathrm{M})$. The positive control was also diluted to the appropriate concentration in Cell Lysis buffer and was treated exactly as the tissue samples were. Incubations at $37^{\circ} \mathrm{C}$ on an orbital shaker were performed for $2 \mathrm{~h}$ before sample absorbance was detected at $405 \mathrm{~nm}$ using a BioTek spectrophotometer.

\subsubsection{Il-1ß protein ELISA}

A mouse IL-1 beta SimpleStep ELISA kit (Abcam, \#ab197742) was used to validate the results of the IL-1 $\beta$ western blot, and detect both the pro-form and cleaved form of IL-1 $\beta$, together. BAT and WAT protein lysates were prepared as described above for western blotting, (n = 4-5 biological replicates for EC, LT and EA). A standard curve was prepared using mouse IL$1 \beta$ lyophilized recombinant protein reconstituted in cell lysis buffer and diluted to set concentrations with sample diluent, as per the manufacturer's recommendations. Following the Bio-Rad method of protein concentration determination, standard curves of WAT and BAT pooled samples were also prepared. The signal from the WAT standard curve did not exceed the signal from the blank at any concentration. By contrast, the standard curve for BAT fell within the signal from the mouse IL-1 $\beta$ lyophilized recombinant protein standard curve, so quantification proceeded for BAT IL-1 $\beta$ only. Then, $500 \mu \mathrm{g}$ of total BAT protein extract from each biological replicate was suspended in $50 \mu \mathrm{L}$ of sample diluent and incubated with $50 \mu \mathrm{L}$ of Antibody Cocktail for 1 hour at room temperature on an orbital shaker. The Antibody Cocktail included mouse IL- $\beta$ Capture and Detector antibodies diluted as described by the manufacturer in Antibody diluent 5BI. Wells were washed $3 \mathrm{X}$ with $350 \mu \mathrm{L}$ wash buffer (diluted 10X in autoclaved water). A 15 min incubation with $100 \mu \mathrm{L}$ of 3,3',5,5'-tetramethylbenzidine (TMB) was terminated by the addition of $100 \mu \mathrm{L}$ of stop solution and absorbance readings were taken at $450 \mathrm{~nm}$ on a BioTek spectrophotometer. 


\subsubsection{Statistical analyses}

Statistical analysis of RT-qPCR experiments involved converting raw Ct values obtained from each PCR run to a linear form using $2^{\wedge-\mathrm{Ct}}$ calculations $(\Delta \mathrm{Ct})$. For every gene of interest, the $\Delta \mathrm{Ct}$ value for each biological replicate was normalized against the $\Delta \mathrm{Ct}$ of the reference gene for the same sample $(\Delta \Delta \mathrm{Ct})$. The reference gene used for WAT was Actal and the geometric mean of the $\Delta \mathrm{Ct}$ values of Tbp and Actg for each sample was used as the reference gene for BAT (Taylor et al., 2019). In the analysis of relative inflammasome transcript levels in BAT, an $n=4$ was used in the statistical analyses of all genes except an outlier was removed in two cases such that an $\mathrm{n}=$ 3 was used in Gsdmd transcript level analysis for the ET time point, and an n $=3$ was used in $I l 18$ transcript level analysis for the IA time point. For all genes analyzed in WAT, an $n=4$ was used for $\mathrm{EC}$ and $\mathrm{ET}$, and LT time points, and an $\mathrm{n}=3$ unique biological replicates was used for EN, LT and EA time points.

Immunoblot bands were imaged using the Chemi-Genius Bioimaging system (Syngene, Frederick, MD) and were quantified using GeneTools software. Subsequently PVDF membranes were stained with Coomassie Blue (0.25\% w:v Coomassie brilliant blue, $7.5 \%$ v:v acetic acid, $50 \%$ methanol) to visualize total protein levels in each lane. Chemiluminescent band density in each lane was then standardized against the summed intensity of a group of Coomassie stained protein bands in the same lane (Eaton et al., 2013). This group was well-separated on the gel from the immunoblot band of interest and was present in all experimental lanes. Since there were too many samples to load on a single gel, the same set of samples from one time point (EC, EN, ET, LT, EA, or IA) was loaded on all three polyacrylamide such that the same sample could be used to judge the relative band density of the other bands. Then, the relative intensity of each band was standardized to the average intensity of the control (EC) bands.

Analysis of the caspase-1 activity assay required that the triplicate absorbance values (technical replicates) detected at $405 \mathrm{~nm}$ were averaged for each of the biological replicates, 
where an $n=5$ was used with the exception of using an $n=4$ for EC (WAT and BAT) and an $n=4$ was used for LT (BAT only). Then, the average absorbance value for each biological replicate was blank subtracted using the average absorbance value of the blank wells, also prepared in triplicate. The blank wells contained Cell Lysis Buffer in lieu of protein, as per the recommendations of the supplier, but were otherwise treated the same as the test wells. The standard curve was used to determine the units of caspase-1 activity in BAT and WAT total protein extracts. The absorbance values from the IL-1 $\beta$ assay were treated the same way, where the standard curve of mouse IL-1 $\beta$ recombinant protein was used to determine the protein concentration of IL-1 $\beta(\mathrm{pg} / \mathrm{mL})$ in the total protein extracts.

For all experiments, all graphs and statistical analyses were completed using RBioplot (Zhang and Storey, 2016). Data are expressed as mean (+/- SEM), with $n=3-5$ for all analyses. A one-way ANOVA with a Tukey post-hoc test was used to determine if there were statistical differences between the relative protein or transcript levels between any of the six time points in immunoblotting and RT-qPCR analysis, or between any of the three time points (EC, LT, and EA) used in relative caspase activity measurements $(\mathrm{p}<0.05)$. For graphing purposes, EC was set to 1 and all other values are shown relative to EC, except for the results of the caspase-1 activity assay and the IL-1 $1 \beta$ assay, where the results are shown as units of enzyme activity, and $\mathrm{pg} / \mathrm{mL}$ of cytokine, respectively. Shared letters between time points indicate no statistical significance and different letters indicate statistical differences with a confidence interval of $95 \%$.

\subsection{Results}

\subsubsection{Relative inflammasome transcript levels in brown and white adipose tissue}

There was a significant increase in the levels of Nlrp3, Casp1 and Illb during interbout arousal (Figure 2.1). Levels of Nlrp3 increased to nearly 2.7-fold of the EC level, whereas both Casp1 and $I l 1 b$ levels increased over 3.1-fold the EC level. Levels of $I l 18$ increased during IA to 
1.4-fold the EC level, but this change was only significant with respect to EN animals, whose average $I l 18$ levels was low, at just over $40 \%$ of the control value. Transcript levels of Pycard did not change. Aim 2 mRNA amounts increased significantly during EN by 2.0 -fold before decreasing during ET to control levels. IA levels of Aim2 were significantly higher than they were during ET and EA, though they were only 1.5-fold the EC level. Finally, Gsdmd, the gene for the protein gasdermin D, increased significantly during EN (by 1.7-fold) before significantly decreasing with respect to EN during ET and LT. Gsdmd levels decreased further during EA and IA to levels between 31 and $46 \%$ of EC.

Few changes in the mRNA levels of inflammasome markers were observed in WAT (Figure 2.2). There were no changes in the relative amounts of Nlrp3, Aim2, Casp1, or Illb. Relative Pycard levels (the gene encoding ASC) increased during ET (by 1.60 -fold \pm 0.16 ) compared to EC and this change was also significant with respect to IA. Gsdmd abundance was also relatively constant during each of the 6 points of the torpor-arousal cycle but there was a significant increase during IA (by 3.15 -fold \pm 0.64 ) with respect to the control, EC. Similarly, Ill 8 mRNA levels increased during IA compared to EC (3.52-fold \pm 0.39$)$ and EN.

\subsubsection{Western blot analysis of key inflammasome proteins in BAT and WAT}

Western blot analysis was more effective at detecting relative inflammasome pathway proteins and quantifying their relative levels in BAT (Figure 2.3) than in the protein-deficient WAT, despite western blotting optimization attempts. Appendix A shows that for almost all the targets included in the current study, only the band or bands of interest (i.e. bands appearing close to the computationally predicted molecular weights) were detected and quantified. Molecular weights are included here and off-target bands are discussed.

At the protein level, NLRP1 was detected as a strong set of two bands appearing between $51 \mathrm{kDa}$ and $62 \mathrm{kDa}$ in 13-lined ground squirrel BAT. The quantification of the more intense band is shown in Figure 2.3, but the quantification of both bands together is shown in Supplemental 
Figure 2.1. No other prominent bands were visible on the blot. At the protein level, NLRP1 levels decreased significantly at all time points except for ET, which was demonstrated to have $70 \%$ of EC value $( \pm 3 \%)$. NLRP1 band densities were only $34-51 \%$ of the EC intensity. However, when both the top, sharp band and the bottom diffuse band are quantified together, there are no significant differences in the levels of NALP1 at any point of the torpor-arousal cycle. The NLRP3 band was detected as a single band at $95 \mathrm{kDa}$ in BAT, similar to the expected value of $106 \mathrm{kDa}$. NLRP3 levels remained relatively constant, except during LT when levels increased to 1.57 -fold \pm 0.43 the EC level, which was statistically significant with respect to EN and ET. A single $45 \mathrm{kDa}$ band was observed for AIM2, similar to the expected molecular weight of 39.5 kDa. AIM2 levels increased during EA with respect to LT, only. Pro-caspase 1 (p42) and procaspase 5 (p45 and p30) protein levels did significantly differ between time points of the torporarousal cycle. Similarly, relative levels of full-length $(\sim 51 \mathrm{kDa})$ and cleaved-forms $(\sim 30 \mathrm{kDa})$ of gasdermin D were unchanging. Cleaved gasdermin D had two very closely associated bands that were quantified together. However, when GSDMD splice events were considered as a ratio of cleaved GSDMD to full-length GSDMD, there was a notable increase in cleaved/full-length gasdermin D during LT (1.75-fold \pm 0.19$)$ and IA (1.71-fold \pm 0.14$)$, compared to EC values. Full length IL-1 $\beta$ and IL-18 (both appearing around $31 \mathrm{kDa}$ ) levels were also differentially regulated in BAT at different points of the torpor-arousal cycle. Computationally predicted $I$. tridecemlineatus IL-18 is $90 \%$ similar in amino acid sequence to mouse IL-18 and it appeared at the correct molecular weight, providing confidence that the correct band was quantified. Pro-IL$1 \beta$ levels were observed to decrease during LT to $48 \%$ of the EN level but there were no statistically significant changes relative to EC. By contrast, pro-IL-18 levels increased during ET (1.42-fold \pm 0.05$)$ and IA (1.53-fold \pm 0.06$)$ compared to EC. The $14 \mathrm{kDa}$ band on the IL-18 western blot was not quantified since it did not appear on some western blots. 
Western blotting was used to quantify the relative levels of gasdermin D and IL-1 $\beta$ in 13lined ground squirrel WAT (Figure 2.4). Full-length and cleaved forms of Gasdermin D levels were unchanged. There were also no statistically significant differences in the ratios of full-length and cleaved gasdermin D band intensities using WAT total protein homogenates. Similarly, IL-1 $\beta$ levels were relatively constant at each point of the torpor-arousal cycle. Full blots for each target with the molecular weight ladders are available in Appendix D. Of note, there was an off-target band on the IL- $1 \beta$ western blots, distant from the region of quantification, at $\sim 62 \mathrm{kDa}$. A protein alignment using Clustal Omega of the antibody epitope used to detect IL-1 $\beta$ (Rattus norvegicus IL-1 $\beta$ protein sequence from amino acids $238-268$ ) with the computationally predicted sequence of IL-1 $\beta$ in I. tridecemlineatus revealed the sequences are highly similar at $80 \%$ sequence identity. An NCBI protein BLAST of the epitope of the antibody showed IL-1 $\beta$ to be the only protein to contain this sequence in ground squirrel, and in a range of other species. This result suggested that the $\sim 62 \mathrm{kDa}$ protein does not have an amino sequence that the primary antibody could have bound to, so it is most likely that the secondary antibody non-specifically targeted this high abundance protein. This is consistent with having to load high concentrations of the protein extracts and optimizing blocking conditions to resolve the IL-1 $\beta$ band at $\sim 30 \mathrm{kDa}$.

\subsubsection{Caspase-1 activity is differentially regulated during deep torpor and upon arousal}

Caspase- 1 activity in BAT was found to increase 1.50 -fold \pm 0.15 during early arousal, relative to the EC control group (Figure 2.5). Specifically, units of caspase-1 activity increased from $3.50 \pm 0.38$ units to $5.27 \pm 0.52$ units. Units of caspase- 1 activity were $4.45 \pm 0.22$ during LT (1.27-fold \pm 0.06 the EC value), which was not different from EC or EA. By contrast, WAT caspase- 1 activity decreased during LT and EA to $67 \% \pm 2 \%$ and $75 \% \pm 7 \%$ of the EC value, respectively. There were $4.26 \pm 0.61$ units of caspase- 1 activity during EC, $3.12 \pm 0.11$ units during LT, and $3.46 \pm 0.34$ units during EA. 


\subsubsection{Interleukin 1 beta levels increase in BAT during arousal}

Total IL-1 $\beta$ (the sum of pro-forms and cleaved IL-1 $\beta$ ) was quantified in $500 \mu \mathrm{g}$ of total BAT from ground squirrels sampled during EC, LT and EA (Figure 2.6) using a different set of antibodies compared to IL-1 $\beta$ detection via western blot. IL-1 $\beta$ levels increased during EA (3.88 $\mathrm{pg} / \mathrm{mL} \pm 0.34)$ relative to $\mathrm{EC}(2.14 \mathrm{pg} / \mathrm{mL} \pm 0.47)$ by 1.81 -fold \pm 0.16 . The concentration of IL- $\beta$ in BAT total protein extract from LT ground squirrels was $3.31 \mathrm{pg} / \mathrm{mL} \pm 0.45$ but this concentration was no different from EC levels.

\subsection{Discussion}

\subsubsection{Inflammasome priming events are more evident in BAT than WAT}

Inflammasome "priming" describes a process where the transcription of protein-coding genes necessary for inflammasome formation and activation (i.e. Nlrp3, Illb) is increased following pattern recognition receptor (PRR) stimulation, which leads to subsequent increases in inflammasome protein levels (D'Espessailles et al., 2018; Wen et al., 2011). Without increasing the expression of Nlrp3 or $I l 1 B$ genes, their respective protein levels would not be high enough to allow for successful inflammasome formation and activity. Thus, RT-qPCR was used to determine if the relative transcript levels of major inflammasome components was different at various points of the torpor-arousal cycle in the 13-lined ground squirrel BAT and WAT. Relative transcript abundance analysis in BAT revealed a consistent increase in the levels of key inflammasome genes (Nlrp3, Casp 1, and Illb) during arousal (IA) relative to the euthermic in the cold room (EC) control group, as well as a significant increase in the mRNA transcript levels of Il18 during arousal, relative to the EN group (Figure 2.1). Temporal alignment in the observed increases in mRNA levels, along with the fact that the expression of these four genes can be stimulated by priming events (D’Espessailles et al., 2018; Schroder et al., 2012), strongly 
suggested that NLRP3 inflammasome priming events could occur during arousal in 13-lined ground squirrel BAT.

AIM2 priming may also be required to elevate AIM2 inflammasome activity, based on experiments that showed AIM2 could detect smaller levels of exogenous DNA if cells were first stimulated with pro-inflammatory lipopolysaccharide (LPS) (Schroder et al., 2012). In this study, priming with LPS did not increase AIM2 protein levels, suggesting that LPS may activate enzymes such as kinases that manage AIM2 post-translational modifications, leading to the increase in sensitivity of AIM2 to smaller levels of DNA (Schroder et al., 2012). As a result, a decrease in Aim2 mRNA transcript levels in ground squirrel BAT during ET and EA relative to EN (Figure 2.1) could suggest that ground squirrel BAT increases Aim2 gene expression during EN or IA to maintain AIM2 proteins levels throughout torpor, which is evident by the relatively unchanging levels of AIM2 protein except during EA (Figure 2.3). The use of PTMs to control AIM2 activity instead of increased AIM2 protein levels would be consistent with a use of this rapidly reversible and energy efficient control over metabolic processes in metabolically suppressed animals as well as the aforementioned study regarding AIM2 sensitivity (Schroder et al., 2012; Storey, 2010). Future experiments may be required to identify the PTMs and the enzymes that regulate them in hibernating ground squirrels. As an aside, Aim 2 transcript levels can be induced by type I and type II interferon (IFN) stimulation of receptors (Liu et al., 2017; Ponomareva et al., 2013), but IFN activity prevents the expression of Nlrp3 and $I l 1 b$ (Schroder et al., 2012). The data suggests that IFNs likely do not stimulate any receptors in ground squirrel BAT since there are no observed increases in Aim 2 transcript levels, and Nlrp3 and Ill 1 transcript levels are increased.

By contrast, analysis of inflammasome transcript levels suggested that inflammasome priming does not occur in 13-lined ground squirrel WAT (Figure 2.2). Gsdmd and $I l 18$ transcript levels increased during IA with respect to EC, but without a coordinated increase in Nlrp3 
transcript levels, these results can only broadly implicate another inflammasome receptor in the regulation of inflammation in ground squirrel WAT. Pycard is the gene expressing the linker protein ASC. The modest increase Pycard transcripts during ET does not temporally align with the increases in Gsdmd and Ill8 transcript levels. Furthermore, using a C57BL/6 cell lines expressing fluorescently labelled ASC, it was shown that expression of ASC alone does not overcome the requirement for NFKB-mediated NLRP3 inflammasome priming events (Bauernfeind et al., 2009). Therefore, it is unlikely that Pycard is upregulated during ET to mediate inflammasome protein-protein interactions and subsequent caspase-1 activation.

The results from ground squirrel BAT are interesting because they suggest PRRs may activate NFאB signaling during arousal in brown fat, promoting NLRP3 inflammasome priming events. NFאB is suggested to be the main transcription factor that gets activated by a priming stimulus to increase the expression of inflammasome receptors such as NLRP3 and interleukins IL-1 $\beta$ and IL-18 (Afonina et al., 2017; Bauernfeind et al., 2009). Preliminary experiments found NFאB may be more active in 13-lined ground squirrel intestinal mucosa compared to BAT but the relative DNA binding capacity of $\mathrm{NF} \kappa \mathrm{B}$ was not reported for all time points of the torpor arousal cycle (Carey et al., 2000). More recently, a multiplex profiling experiment on control and torpid

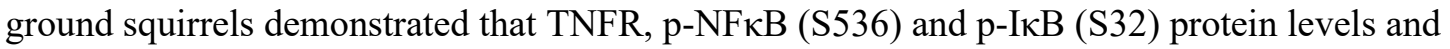
NFkB DNA binding capacity are maintained during torpor in ground squirrel BAT, while c-Myc, a gene downstream of NFאB transcriptional activation was increased during torpor (Hadj-Moussa et al., 2020). Phosphorylation status of NFאB and its mediators $(\mathrm{IKK} \alpha / \beta, \mathrm{I} \kappa \mathrm{B})$ predict the activation status of the NFאB signal transduction pathway, so further experiments are required to conclusively determine if $\mathrm{NF \kappa B}$ signaling is more active in BAT and less active in WAT, as the results of the RT-qPCR experiments suggest. 


\subsubsection{Inflammasome formation more likely occurs in BAT than WAT}

Ground squirrel BAT differentially expressed all three of the inflammasome receptors investigated (NLRP1, NLRP3, and AIM2), at different points of the torpor-arousal cycle (Figure 2.3). NLRP1 was discovered in BAT appearing as two bands between $51 \mathrm{kDa}$ and $62 \mathrm{kDa}$

(Figure 2.3b), at a molecular weight much lower than computationally predicted. NLRP1 was not detected in WAT. However, human NLRP1 has been observed between $40 \mathrm{kDa}$ and $65 \mathrm{kDa}$ using another antibody that also binds the N-terminal of NLRP1 (e.g. Novus Biologicals, \#NBP154899). Post-translational cleavage or alternative splicing may explain its truncated size, and the double band pattern may be explained by post-translational modifications. Indeed, NLRP1 cleavage can result in this protein becoming N-terminally destabilized and degraded by the proteasome such that the C-terminal CARD is released and used as a platform for caspase- 1 activation (Sandstrom et al., 2019). However, until this phenomenon is explored more thoroughly in the BAT of hibernating ground squirrels, a modest interpretation of the decrease in NLRP1 protein levels at all time points of the torpor-arousal cycle except for ET, relative to EC, would be that NLRP1 inflammasomes are not activated in BAT in hibernating ground squirrels.

By contrast, the relative levels of NLRP3 increased during LT (significant compared to EN and ET), and AIM2 protein levels increased during EA (significant compared to LT) (Figure 2.3). These results do not match the relative transcript level data, suggesting there may be a lag between transcription and protein translation of inflammasome components, resulting in inflammasome formation at specific time points of the torpor-arousal cycle in hibernating ground squirrel BAT. Specifically, the RT-qPCR results indicate that priming stimuli may increase during arousal, based on increased levels of Nlrp3 and other NLRP3 inflammasome components during arousal (Figure 2.1). As ground squirrels use non-shivering thermogenesis to rapidly rewarm in the arousal period, oxygen intake and metabolic respiration increases can generate ROS, which could react with protein, lipids, and DNA to create endogenous stimulators of PRRs. 
This idea is supported by increases in markers of oxidative stress such as lipid peroxides and protein carbonyls in the BAT of Arctic ground squirrels arousing or aroused from torpor, relative to hibernating ground squirrels (Orr et al., 2009). Furthermore, it has been proposed that arousals may be used to mobilize innate immune cells and normalize the levels of any proinflammatory and/or ROS-damaged proteins/lipids/polynucleotides that accumulated during torpor (Bouma et al., 2013; Cogut et al., 2017; D’Alessandro et al., 2017; Sahdo et al., 2013). Thus, NLRP3 priming may occur during arousal when PRRs are likely triggered, but the nascent Nlrp3 transcripts are likely not mobilized for translation until they are required in deep torpor, when activating stimuli may be increased.

One clear activator of NLRP3 inflammasome activation is potassium efflux (He et al., 2016; Santoni et al., 2015). In BAT thermogenesis, $\mathrm{K}^{+}$efflux can occur following the release of intracellular $\mathrm{Ca}^{2+}$ stores, which can happen following the stimulation of adrenergic receptors as hibernators rewarm to normothermia (Cannon and Nedergaard, 2004; Chen et al., 2017). Briefly, non-shivering thermogenesis (NST) in the brown adipocyte is stimulated by noradrenaline acting on highly abundant adrenergic receptors, namely $\beta 3$-adrenergic or $\alpha 1$-adrenergic receptors.

Reviewed thoroughly elsewhere (Ballinger and Andrews, 2018; Cannon and Nedergaard, 2004), $\beta 3$-adrenergic receptors signaling results in the uncoupling of oxidative phosphorylation and the dissipation of chemical energy as heat. By contrast, $\alpha 1$-adrenergic receptor signaling results in the production of phosphatidylinositol 4,5-bisphosphate (PIP2) (Nånberg and Putney, 1986). PIP2 is broken into inositol 1,4,5-triphosphate (IP3) and diglycerides. Interestingly, transcriptomics data from 13-lined ground squirrel BAT confirms that the enzyme involved in converting PIP2 to diacylglycerol and IP3 is upregulated during LT and IA (Hampton et al., 2013), which suggests $\alpha 1$-adrenergic receptor stimulation could increase PIP2 metabolism in hibernator BAT. Downstream of this event, diacylglycerol activates protein kinase C (PKC), which like PKA, phosphorylates CREB, the transcription factor that increases the expression of uncoupling protein 
1 (UCP1). (Cannon and Nedergaard, 2004). Indeed, CREB is differentially phosphorylated during LT and IA in the 13-lined ground squirrel BAT, but not WAT (Rouble et al., 2014), supporting a role for $\beta 3$ and/or $\alpha 1$-mediated signaling during LT and IA in BAT, but not WAT. Secondly, IP3 triggers the release of $\mathrm{Ca}^{2+}$ from intracellular $\mathrm{Ca}^{2+}$ stores, leading to increases cytoplasmic $\mathrm{Ca}^{2+}$ levels and subsequent $\mathrm{Cl}^{-}$and $\mathrm{K}^{+}$efflux (Cannon and Nedergaard, 2004). The uncontrolled release of endoplasmic reticulum $\mathrm{Ca}^{2+}$ stores can have a grave effect on cell viability, through sustained plasma membrane depolarization and $\mathrm{Ca}^{2+}$-stimulation of mitochondrial permeability transition pore opening. Therefore, mammals have mechanisms to sense and respond to IP3mediated release of intracellular $\mathrm{Ca}^{2+}$. For instance, phosphorylated Bcl-2 levels were increased in 13-lined ground squirrel BAT during torpor (Rouble et al., 2013), which may be useful since Bcl-2 can reduce apoptosis by preventing excessive IP3-mediated release of intracellular $\mathrm{Ca}^{2+}$ stores (Rong et al., 2008). Calcium is a known inducer of NLRP3 but not AIM2 inflammasome activation, where the inhibition of the IP3 receptor prevents intracellular calcium release and prevents NLRP3 inflammasome activation (He et al., 2016). Therefore, the increase in p-Bcl-2 during torpor may attenuate intracellular calcium release, resulting in reduced activation of $\mathrm{Ca}^{2+-}$ mediated $\mathrm{K}^{+}$channels and subsequent $\mathrm{K}^{+}$efflux. Consistent with decreases in NLRP3 inflammasome-mediated IL-1 $\beta$ maturation upon Bcl-2 overexpression (Shimada et al., 2012), no statistically significant changes in caspase- 1 activity during LT relative to EC suggest that $\mathrm{K}^{+}$ efflux is insufficient during LT to promote NLRP3 activation in ground squirrel BAT (Figure 2.5). This is also consistent with relatively low levels of NST during LT, relative to NST during arousal, that may be required to maintain a thermogenic set-point $\left(\mathrm{T}_{\text {set }}\right)$ within a narrow range (Geiser, 2004; Staples, 2014).

However, it is important to note that the ratio of cleaved GSDMD to full-length GSDMD increased during LT, and pro-IL-18 levels decreased during LT relative to ET suggesting interleukin maturation. Unfortunately, the p14 IL-18 band did not appear on all membranes to 
quantify what is possibly the cleavage product of pro-IL-18. Notably, this protein is about $3 \mathrm{kDa}$ smaller than the anticipated cleavage product, suggesting ground squirrels may produce alternatively spliced IL-18 during the torpor-arousal cycle, which may alter the location of caspase-1 cleavage on this protein. Overall, the data suggested NLRP3 inflammasomes may form and activate caspase-1 in some ground squirrels. Perhaps the low levels of NST required to maintain a low $\mathrm{T}_{\text {set }}$ during LT are similar to the levels of NST used in euthermic ground squirrels that are active in a cold room - e.g. some NST occurs but the level is not sufficiently different to invoke different magnitudes of $\mathrm{Ca}^{2}$ and subsequent $\mathrm{K}^{+}$release from brown adipocytes. Indeed, low levels of $\mathrm{K}^{+}$efflux from brown adipocytes during LT may be protective. $\mathrm{K}^{+}$efflux has been postulated to trigger the normalization of the plasma membrane polarization following NSTmediated ion flux, as well as reduce the overconsumption of cellular resources by mediating cAMP production and lipolysis (Chen et al., 2017). For example, the Potassium Two Pore Domain Channel Subfamily K Member 3 (KCNK3) potassium channel simultaneously increased $\mathrm{K}^{+}$efflux from brown adipocytes and reduced the influx of extracellular $\mathrm{Ca}^{2+}$ (and subsequent lipolysis and apoptosis) by modulating the activity of voltage-gated calcium channels (Cannon and Nedergaard, 2004; Chen et al., 2017). Knocking out the KCNK3 $\mathrm{K}^{+}$channel in brown adipocytes lead to increased levels of cAMP, active HSL, and glycerol, suggesting increased lipolysis (Chen et al., 2017). It is possible that thermogenesis to maintain $\mathrm{T}_{\text {set }}$ during torpor in addition to thermogenesis during arousal may trigger $\mathrm{K}^{+}$efflux and possibly, low levels of NLRP3 inflammasome activation in hibernating ground squirrel BAT.

As previously mentioned, AIM2 activation does not require elaborate priming events like NLRP3 inflammasomes. Instead, AIM2 inflammasomes are more sensitive to the presence of their ligands (e.g. damaged DNA in the nucleus, nuclear or mitochondrial DNA in the cytoplasm, or foreign DNA) when there is a previous insult to the cell (e.g. LPS in the case of bacterial infection) (Bae et al., 2019; Cunha et al., 2017). Since AIM2 levels increased during EA, it is 
possible that there was some level of mitochondrial or nuclear disruption, instigating the release of DNA into the cytoplasm. Alternatively, an increase in aerobic metabolism as hibernators began to rapidly warm could have sparked a release of ROS, especially in the mitochondria, resulting in ROS-mediated DNA damage that AIM2 would be able to detect. Gsdmd transcript levels decreased during the arousal period (EA and IA) in BAT (Figure 2.1), which suggested that transcripts were being translated into protein to maintain total protein levels. At the protein level, full-length and cleaved gasdermin D protein levels were individually maintained in BAT throughout the torpor bout (Figure 2.3). However, the ratio of cleaved to full-length GSDMD increased during arousal, suggesting AIM2 may have indeed formed active inflammasomes. Since inflammasome assembly results in cleaved GSDMD-mediated pore formation, which can allow for the release of IL-1 $\beta$ and IL-18 from the cell (Platnich and Muruve, 2019), a decrease in pro-interleukin could represent a release of active (cleaved) IL-1 $\beta$ or IL-18 into the intercellular space. Indeed, total IL-1 $\beta$ concentration (pro- and cleaved forms) increased significantly during EA (Figure 2.6), despite relatively no changes in the levels of pro-IL-1 $\beta$ protein levels (Figure 2.3). The C-terminal region of the computationally predicted I. tridecemlineatus IL-1 $\beta$ is highly similar in sequence identity with the C-terminal region of the Mus musculus IL-1 $\beta$, which the Abcam ELISA antibodies were raised against. This region of the protein does not appear to have major sequence similarity with other proteins, having performed an NCBI BLAST search, providing confidence that this ELISA is specific for IL-1 $\beta$. The antibodies used in the Abcam ELISA are not validated for western blotting, which is important because antibodies used in the ELISA would recognize epitopes on folded proteins, and this epitope may not be present on a linearized protein in a western blot experiment. Given that we did not confirm the molecular weight of IL-1 $\beta$ from the ELISA experiment, these results are interpreted with caution, but seem to agree with the pro-IL-1 $\beta$ western blotting results. Together, the results suggests that inflammasomes may form in BAT such that pro-IL-1 $\beta$ is increasingly processed into the cleaved, active form, for signaling during arousal. Strong support for AIM2 inflammasome formation and 
interleukin processing in 13-lined ground squirrel BAT comes from the significant increase in caspase-1 activity levels during EA relative to EC.

Interestingly, mice deficient in AIM2 increase adipogenesis, have dysfunctional BAT function including high levels of pro-inflammatory markers and have lower energy expenditure (Gong et al., 2019). With this in mind, AIM2 inflammasomes may promote proper functioning of BAT in arousing hibernators, perhaps by recruiting white blood cells via activated IL-18 and/or IL-1 $\beta$ to remove sources of cell stress (e.g. phagocytosis of cells undergoing pyroptosis) during arousal. Indeed, previous studies on ground squirrels have shown ROS and pro-inflammatory proteins accumulated during torpor are likely normalized during interbout arousal, based on metabolite measurements in liver comparing LT with EN, the time point used to estimate the completion of damage marker normalization during IA (Serkova et al., 2007). The few studies that have investigated markers of inflammatory or immune signaling in hibernators tend to support a role for immune and inflammatory responses during arousals from torpor (Bohr et al., 2014; Cogut et al., 2017; Prendergast et al., 2002; Serkova et al., 2007). For example, the lungs of interbout aroused ground squirrels increase the abundance of E-selectin relative to summer and hibernating ground squirrels, suggesting a role for adhesion molecules in targeting immune cells to areas of local damage (Bohr et al., 2014; Roth Flach et al., 2013). The same ground squirrel study noted low levels of pro-inflammatory proteins such as suppressor of cytokine signaling (SOCS) during torpor, but the levels of these proteins remained unchanged in interbout arousal with respect to the summer control (Bohr et al., 2014), suggesting a resumption of damage sensing and response during arousal periods. Similarly, IL-1 $\beta$ levels increased early in the arousal process in the brain of Syrian hamster but after several hours at interbout arousal, the levels of this cytokine were normalized (Cogut et al., 2017). This result in particular reinforces the need to study inflammasome activation in different tissues to determine and different species of 
hibernator to determine how species capable of metabolic suppression sense and respond to danger signals to limit tissue damage.

Select tissues in the hibernator may not acquire sufficient damage to warrant an activation of inflammasomes or other inflammatory responses at levels of inflammasome activation seen in mammals defending against pathogens. Experiments contrasting the magnitude of inflammasome activation in hibernators exposed to exogenous stressors vs. natural torpor are warranted. However, in the current study that characterized the natural inflammasome signaling response during torpor, the data suggests endogenous triggers of inflammasome activation may exist in BAT and are less likely to spur a response in WAT in hibernating ground squirrels. The caspase-1 activity assay suggested a role for inflammasomes in BAT during arousal, but a significant decrease in caspase-1 activity in WAT during LT and EA suggests the opposite. The immunoblotting results also suggested inflammasome activation may be suppressed over the torpor-arousal cycle in WAT. There were no significant differences in GSDMD levels when assessing the levels of full-length GSDMD, cleaved GSDMD, or the cleaved to full-length GSDMD ratio, at any point of the torpor-arousal cycle (Figure 2.4). Secondly, pro-IL-1 $\beta$ levels did not change at any time point, relative to EC. It was predicted that hypertrophied WAT cells may be more sensitive to natural endogenous damage sensed throughout a torpor-bout, but the data indicates that inflammation is unlikely more pronounced over a torpor bout relative to a prehibernating euthermic control group housed at the same room temperature. Since the ablation of core inflammasome components protect mice fed high-fat diets from obesity-induced adipose dysfunction and insulin resistance (Gong et al., 2019), it is possible that hibernators inhibit the activation of inflammasomes (with the most compelling evidence of this coming from the caspase-1 activity assay) in WAT to maintain adipose function over several months of hibernation. Future studies should investigate the differences in WAT inflammasome signaling comparing post-reproductive and pre-hyperphagic summer active ground squirrels with post- 
hyperphagic/pre-hibernating ground squirrels, the latter of which are reversibly insulin resistant (Martin, 2008; Wu et al., 2013).

\subsection{Conclusion}

The experiments performed herein suggest that NLRP3 and AIM2 but not NLRP1 inflammasomes may play a role in sensing endogenous damage in BAT during a torpor-arousal bout and then responding to that damage by activating an inflammatory response. More specifically, NLRP3 inflammasomes may be activated during torpor by changes in NST-mediated calcium and potassium flux, and AIM2 inflammasomes may be triggered during arousal by oxidative damage to mitochondrial DNA or mitochondrial/perinuclear membrane rupture, releasing polynucleotides into the cytosol. By contrast, there was little evidence that an inflammatory response is invoked in hibernating ground squirrel WAT, suggesting that the hypothesis that chronic hypertrophy of the adipocytes in this endocrine organ does not cause additional stress in this tissue in metabolically suppressed ground squirrels. Instead, it may be valuable to assess inflammasome activation during the hyperphagic period, when adipocytes are being acutely stressed by hypertrophy. Together, these experiments support the idea that select tissues of the hibernating ground squirrel use molecular signaling pathways to sense natural changes in cellular homeostasis during a torpor-arousal cycle. Furthermore, these experiments suggest that inflammatory signaling amplified by inflammasome activation likely has a role during arousal to eliminate endogenous damage associated molecular patterns in BAT. 


\subsection{References}

Afonina, I.S., Zhong, Z., Karin, M., Beyaert, R., 2017. Limiting inflammation - The negative regulation of NF-kB and the NLRP3 inflammasome. Nat. Immunol. 18, 861-869. https://doi.org/10.1038/ni.3772

Alfonso-Loeches, S., Ureña-Peralta, J., Morillo-Bargues, M.J., Gómez-Pinedo, U., Guerri, C., 2016. Ethanol-induced TLR4/NLRP3 neuroinflammatory response in microglial cells promotes leukocyte infiltration across the BBB. Neurochem. Res. 41, 193-209. https://doi.org/10.1007/s11064-015-17605

Badran, M., Golbidi, S., Devlin, A., Ayas, N., Laher, I., 2014. Chronic intermittent hypoxia causes endothelial dysfunction in a mouse model of diet-induced obesity. Sleep Med. 15, 596-602. https://doi.org/10.1016/j.sleep.2014.01.013

Bae, J.H., Jo, S.I., Kim, S.J., Lee, J.M., Jeong, J.H., Kang, J.S., Cho, N.-J., Kim, S.S., Lee, E.Y., Moon, J.S., 2019. Circulating cell-free mtDNA contributes to AIM2 inflammasome-mediated chronic inflammation in patients with type 2 diabetes. Cells 8, 328. https://doi.org/10.3390/cells8040328

Ballinger, M.A., Andrews, M.T., 2018. Nature's fat-burning machine: brown adipose tissue in a hibernating mammal. J. Exp. Biol. 221, jeb162586. https://doi.org/10.1242/jeb.162586

Ballinger, M.A., Hess, C., Napolitano, M.W., Bjork, J.A., Andrews, M.T., 2016. Seasonal changes in brown adipose tissue mitochondria in a mammalian hibernator: from gene expression to function. Am. J. Physiol. - Regul. Integr. Comp. Physiol. 311, R325-R336. https://doi.org/10.1152/ajpregu.00463.2015

Bauernfeind, F.G., Horvath, G., Stutz, A., Alnemri, E.S., MacDonald, K., Speert, D., Fernandes-Alnemri, T., Wu, J., Monks, B.G., Fitzgerald, K.A., Hornung, V., Latz, E., 2009. Cutting Edge: NF-кB Activating Pattern Recognition and Cytokine Receptors License NLRP3 Inflammasome Activation by Regulating NLRP3 Expression. J. Immunol. 183, 787-791. https://doi.org/10.4049/jimmunol.0901363

Bohr, M., Brooks, A.R., Kurtz, C.C., 2014. Hibernation induces immune changes in the lung of 13-lined ground squirrels (Ictidomys tridecemlineatus). Dev. Comp. Immunol. 47, 178-184. https://doi.org/10.1016/j.dci.2014.07.011

Bouma, H.R., Henning, R.H., Kroese, F.G.M., Carey, H. V., 2013. Hibernation is associated with depression of T-cell independent humoral immune responses in the 13-lined ground squirrel. Dev. Comp. Immunol. 39, 154-160. https://doi.org/10.1016/j.dci.2012.11.004

Boyer, B.B., Barnes, B.M., Lowell, B.B., Grujic, D., 1998. Differential regulation of uncoupling protein gene homologues in multiple tissues of hibernating ground squirrels. Am. J. Physiol. 275, R1232-8.

Cannon, B., Nedergaard, J., 2004. Brown adipose issue: Function and physiological significance. Physiol. Rev. 84, 277-359. https://doi.org/10.1152/physrev.00015.2003

Carey, H. V, Frank, C.L., Seifert, J.P., 2000. Hibernation induces oxidative stress and activation of NF-kB in ground squirrel intestine. J. Comp. Physiol. B. 170, 551-9. https://doi.org/10.1007/s003600000135

Chen, Y., Zeng, X., Huang, X., Serag, S., Woolf, C.J., Spiegelman, B.M., 2017. Crosstalk between KCNK3-mediated ion current and adrenergic signaling regulates adipose thermogenesis and obesity. Cell 171, 836-848.e13. https://doi.org/10.1016/j.cell.2017.09.015

Cogut, V., Bruintjes, J.J.J., Eggen, B.J.L.J.L., van der Zee, E.A.A., Henning, R.H.H., 2017. Brain inflammatory cytokines and microglia morphology changes throughout hibernation phases in Syrian hamster. Brain. Behav. Immun. 68, 17-22. https://doi.org/10.1016/j.bbi.2017.10.009

Cunha, L.D., Silva, A.L.N., Ribeiro, J.M., Mascarenhas, D.P.A., Quirino, G.F.S., Santos, L.L., Flavell, R.A., Zamboni, D.S., 2017. AIM2 engages active but unprocessed caspase-1 to induce noncanonical activation of the NLRP3 inflammasome. Cell Rep. 20, 794-805. https://doi.org/10.1016/j.celrep.2017.06.086 
D’Alessandro, A., Nemkov, T., Bogren, L.K., Martin, S.L., Hansen, K.C., 2017. Comfortably numb and back: plasma metabolomics reveals biochemical adaptations in the hibernating 13-Lined ground squirrel. J. Proteome Res. 16, 958-969. https://doi.org/10.1021/acs.jproteome.6b00884

D’Espessailles, A., Mora, Y.A., Fuentes, C., Cifuentes, M., 2018. Calcium-sensing receptor activates the NLRP3 inflammasome in LS14 preadipocytes mediated by ERK1/2 signaling. J. Cell. Physiol. 233, 6232-6240. https://doi.org/10.1002/jcp.26490

Eaton, S.L., Roche, S.L., Llavero Hurtado, M., Oldknow, K.J., Farquharson, C., Gillingwater, T.H., Wishart, T.M., 2013. Total protein analysis as a reliable loading control for quantitative fluorescent western blotting. PLoS One 8, 1-9. https://doi.org/10.1371/journal.pone.0072457

Evavold, C.L., Kagan, J.C., 2018. How inflammasomes inform adaptive immunity. J. Mol. Biol. 430, $217-$ 237. https://doi.org/10.1016/j.jmb.2017.09.019

Frank, C.L., Storey, K.B., 1995. The optimal depot fat composition for hibernation by golden-mantled ground squirrels (Spermophilus lateralis). J. Comp. Physiol. B 164, 536-542. https://doi.org/10.1007/BF00261394

Geiser, F., 2004. Metabolic rate and body temperature reduction during hibernation and daily torpor. Annu. Rev. Physiol. 66, 239-74. https://doi.org/10.1146/annurev.physiol.66.032102.115105

Gong, Z., Zhang, X., Su, K., Jiang, R., Sun, Z., Chen, W., Forno, E., Goetzman, E.S., Wang, J., Dong, H.H., Dutta, P., Muzumdar, R., 2019. Deficiency in AIM2 induces inflammation and adipogenesis in white adipose tissue leading to obesity and insulin resistance. Diabetologia 62, 2325-2339. https://doi.org/10.1007/s00125-019-04983-x

Hampton, M., Melvin, R.G., Andrews, M.T., 2013. Transcriptomic analysis of brown adipose tissue across the physiological extremes of natural hibernation. PLoS One 8, 1-12.

https://doi.org/10.1371/journal.pone.0085157

Harker, C.T., Webb, R.C., 1987. Effect of cooling on vascular smooth muscle from the 13-lined ground squirrel. Cryobiology 24, 74-81. https://doi.org/10.1016/0011-2240(87)90009-5

He, Y., Hara, H., Núñez, G., 2016. Mechanism and regulation of NLRP3 inflammasome activation. Trends Biochem. Sci. 41, 1012-1021. https://doi.org/10.1016/j.tibs.2016.09.002

Liu, F., Niu, Q., Fan, X., Liu, C., Zhang, J., Wei, Z., Hou, W., Kanneganti, T.-D., Robb, M.L., Kim, J.H., Michael, N.L., Sun, J., Soong, L., Hu, H., 2017. Priming and activation of inflammasome by Canarypox virus vector ALVAC via the cGAS/IFI16-STING-Type I IFN pathway and AIM2 sensor. J. Immunol. 199, 3293-3305. https://doi.org/10.4049/jimmunol.1700698

Logan, S.M., Storey, K.B., 2018. Pro-inflammatory AGE-RAGE signaling is activated during arousal from hibernation in ground squirrel adipose. PeerJ 6, e4911. https://doi.org/10.7717/peerj.4911

Logan, S.M., Tessier, S.N., Tye, J., Storey, K.B., 2016. Response of the JAK-STAT pathway to mammalian hibernation in 13-lined ground squirrel striated muscle. Mol. Cell. Biochem. 414, 115127. https://doi.org/10.1007/s11010-016-2665-6

Martin, S.L., 2008. Mammalian hibernation: a naturally reversible model for insulin resistance in man? Diabetes Vasc. Dis. Res. 5, 76-81. https://doi.org/10.3132/dvdr.2008.013

McMullen, D.C., Hallenbeck, J.M., 2010. Regulation of Akt during torpor in the hibernating ground squirrel, Ictidomys tridecemlineatus. J. Comp. Physiol. B Biochem. Syst. Environ. Physiol. 180, $927-$ 934. https://doi.org/10.1007/s00360-010-0468-8

Mitchell, P.S., Sandstrom, A., Vance, R.E., 2019. The NLRP1 inflammasome : new mechanistic insights and unresolved mysteries. Curr. Opin. Immunol. 60, 37-45. https://doi.org/10.1016/j.coi.2019.04.015

Nånberg, E., Putney, J., 1986. a1-Adrenergic activation of brown adipocytes leads to an increased formation of inositol polyphosphates. FEBS Lett. 195, 319-322. https://doi.org/10.1016/0014- 


\section{3(86)80185-5}

Olea, E., Agapito, M.T., Gallego-Martin, T., Rocher, A., Gomez-Niño, A., Obeso, A., Gonzalez, C., Yubero, S., 2014. Intermittent hypoxia and diet-induced obesity: effects on oxidative status, sympathetic tone, plasma glucose and insulin levels, and arterial pressure. J. Appl. Physiol. 117, 706719. https://doi.org/10.1152/japplphysiol.00454.2014

Orr, A.L., Lohse, L.A., Drew, K.L., Hermes-Lima, M., 2009. Physiological oxidative stress after arousal from hibernation in Arctic ground squirrel. Comp. Biochem. Physiol. 153, 213-221. https://doi.org/10.1016/j.cbpa.2009.02.016.Physiological

Pellissier, F., Glogowskib, C.M., Heinemannb, S.F., Balliveta, M., Ossipowa, V., 2006. Lab assembly of a low-cost, robust SYBR green buffer system for quantitative real-time polymerase chain reaction. Anal. Biochem. 350, 310-312. https://doi.org/10.1016/j.ab.2005.12.002

Platnich, J.M., Muruve, D.A., 2019. NOD-like receptors and inflammasomes: A review of their canonical and non-canonical signaling pathways. Arch. Biochem. Biophys. 670, 4-14. https://doi.org/10.1016/j.abb.2019.02.008

Ponomareva, L., Liu, H., Duan, X., Dickerson, E., Shen, H., Panchanathan, R., Choubey, D., 2013. AIM2, an IFN-inducible cytosolic DNA sensor, in the development of benign prostate hyperplasia and prostate cancer. Mol. Cancer Res. 11, 1193-1202. https://doi.org/10.1158/1541-7786.MCR-13-0145

Prendergast, B.J., Freeman, D.A., Zucker, I., Nelson, R.J., 2002. Periodic arousal from hibernation is necessary for initiation of immune responses in ground squirrels. Am. J. Physiol. - Regul. Integr. Comp. Physiol. 282, 1054-1062. https://doi.org/10.1152/ajpregu.00562.2001

Rong, Y.-P., Aromolaran, A.S., Bultynck, G., Zhong, F., Li, X., McColl, K., Matsuyama, S., Herlitze, S., Roderick, H.L., Bootman, M.D., Mignery, G.A., Parys, J.B., De Smedt, H., Distelhorst, C.W., 2008. Targeting Bcl-2-IP3 receptor interaction to reverse Bcl-2's inhibition of apoptotic calcium signals. Mol. Cell 31, 255-265. https://doi.org/10.1016/j.molcel.2008.06.014

Roth Flach, R.J., Matevossian, A., Akie, T.E., Negrin, K.A., Paul, M.T., Czech, M.P., 2013. ß3-Adrenergic receptor stimulation induces E-selectin-mediated adipose tissue inflammation. J. Biol. Chem. 288, 2882-2892. https://doi.org/10.1074/jbc.M112.412346

Rouble, A.N., Hefler, J., Mamady, H., Storey, K.B., Tessier, S.N., 2013. Anti-apoptotic signaling as a cytoprotective mechanism in mammalian hibernation. PeerJ 1, e29. https://doi.org/10.7717/peerj.29

Rouble, A.N., Tessier, S.N., Storey, K.B., 2014. Characterization of adipocyte stress response pathways during hibernation in 13-lined ground squirrels. Mol. Cell. Biochem. 393, 271-282. https://doi.org/10.1007/s11010-014-2070-y

Sahdo, B., Evans, A.L., Arnemo, J.M., Fröbert, O., Särndahl, E., Blanc, S., 2013. Body temperature during hibernation is highly correlated with a decrease in circulating innate immune cells in the brown bear (Ursos arctos): A common feature among hibernators? Int. J. Med. Sci. 10, 508-514. https://doi.org/10.7150/ijms.4476

Sandstrom, A., Mitchell, P.S., Goers, L., Mu, E.W., Lesser, C.F., Vance, R.E., 2019. Functional degradation: A mechanism of NLRP1 inflammasome activation by diverse pathogen enzymes. Science (80-. ). 364. https://doi.org/10.1126/science.aau1330

Santoni, G., Cardinali, C., Morelli, B.B., Santoni, M., Nabissi, M., Amantini, C., 2015. Danger- and pathogen-associated molecular patterns recognition by pattern-recognition receptors and ion channels of the transient receptor potential family triggers the inflammasome activation in immune cells and sensory neurons. J. Neuroinflammation 12, 1-10. https://doi.org/10.1186/s12974-015-0239-2

Schroder, K., Sagulenko, V., Zamoshnikova, A., Richards, A.A., Cridland, J.A., Irvine, K.M., Stacey, K.J., Sweet, M.J., 2012. Acute lipopolysaccharide priming boosts inflammasome activation independently of inflammasome sensor induction. Immunobiology 217, 1325-1329. 
https://doi.org/10.1016/j.imbio.2012.07.020

Serkova, N.J., Rose, J.C., Epperson, L.E., Carey, H. V, Martin, S.L., 2007. Quantitative analysis of liver metabolites in three stages of the circannual hibernation cycle in 13-lined ground squirrels by NMR. Physiol. Genomics 31, 15-24. https://doi.org/10.1152/physiolgenomics.00028.2007

Shimada, K., Crother, T.R., Karlin, J., Dagvadorj, J., Chiba, N., Chen, S., Ramanujan, V.K., Wolf, A.J., Vergnes, L., Ojcius, D.M., Rentsendorj, A., Vargas, M., Guerrero, C., Wang, Y., Fitzgerald, K.A., Underhill, D.M., Town, T., Arditi, M., 2012. Oxidized mitochondrial DNA activates the NLRP3 inflammasome during apoptosis. Immunity 36, 401-414.

https://doi.org/10.1016/j.immuni.2012.01.009

Staples, J.F., 2014. Metabolic suppression in mammalian hibernation: the role of mitochondria. J. Exp. Biol. 217, 2032-2036. https://doi.org/10.1242/jeb.092973

Storey, K.B., 2010. Out cold: Biochemical regulation of mammalian hibernation - A mini-review. Gerontology 56, 220-230. https://doi.org/10.1159/000228829

Wang, L.C.H., Lee, T.F., 1996. Torpor and hibernation in mammals: metabolic, physiological, and biochemical adaptations, in: Pappenheimer, J.R., Fregly, M.J., Blatties, C.M. (Eds.), Handbook of Physiology - Environmental Physiology. Oxford University Press, Oxford, UK, pp. 507-532. https://doi.org/10.1002/cphy.cp040122

Wang, P., Walter, R.D., Bhat, B.G., Florant, G.L., Coleman, R.A., 1997. Seasonal changes in enzymes of lipogenesis and triacylglycerol synthesis in the golden-mantled ground squirrel (Spermophilus lateralis). Comp. Biochem. Physiol. Part B Biochem. Mol. Biol. 118, 261-267. https://doi.org/10.1016/S0305-0491(97)00102-8

Webb, C.L., Milsom, W.K., 2017. Effects of low temperature on breathing pattern and ventilatory responses during hibernation in the golden-mantled ground squirrel. J. Comp. Physiol. B 187, 793802. https://doi.org/10.1007/s00360-017-1079-4

Wen, H., Gris, D., Lei, Y., Jha, S., Zhang, L., Huang, M.T.H., Brickey, W.J., Ting, J.P.Y., 2011. Fatty acidinduced NLRP3-ASC inflammasome activation interferes with insulin signaling. Nat. Immunol. 12, 408-415. https://doi.org/10.1038/ni.2022

Wickler, S.J., Hoyt, D.F., van Breukelen, F., 1991. Disuse atrophy in the hibernating golden-mantled ground squirrel, Spermophilus lateralis. Am. J. Physiol. Integr. Comp. Physiol. 261, R1214-R1217. https://doi.org/10.1152/ajpregu.1991.261.5.R1214

Wu, C.-W., Biggar, K.K., Storey, K.B., 2013. Biochemical adaptations of mammalian hibernation: exploring squirrels as a perspective model for naturally induced reversible insulin resistance. Brazilian J. Med. Biol. Res. 46, 1-13. https://doi.org/10.1590/1414-431X20122388

Young, R.A., Salans, L.B., Sims, E.A.H., 1982. Adipose tissue cellularity in woodchucks: Effects of season and captivity at an early age. J. Lipid Res. 23, 887-892.

Zhang, J., Storey, K.B., 2016. RBioplot: an easy-to-use R pipeline for automated statistical analysis and data visualization in molecular biology and biochemistry. PeerJ 4, e2436. https://doi.org/10.7717/peerj.2436

Zhu, Q., Glazier, B.J., Hinkel, B.C., Cao, J., Liu, L., Liang, C., Shi, H., 2019. Neuroendocrine regulation of energy metabolism involving different types of adipose tissues. Int. J. Mol. Sci. 20, 2707. https://doi.org/10.3390/ijms20112707 


\subsection{Figures}

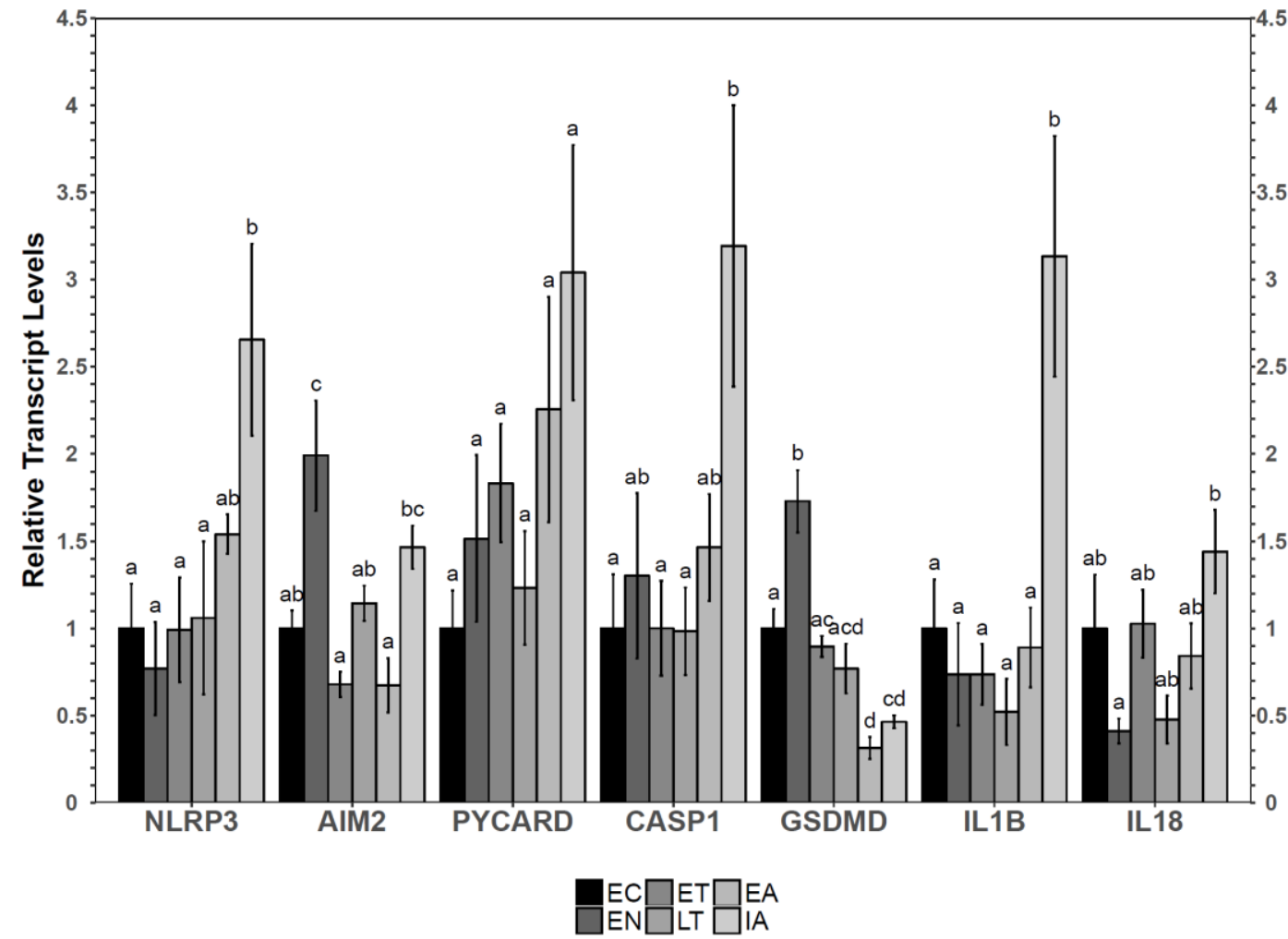

Figure 2.1: Relative transcript levels of key inflammasome targets using brown adipose tissue (BAT) sampled from 13-lined ground squirrels at 6 different points of the torpor-arousal cycle (EC, euthermic in the cold room; EN, entrance into torpor; ET, early torpor, LT, late torpor; EA, early arousal; IA, interbout arousal). Transcript levels of each gene were normalized against the sample-specific abundance of reference genes TATA-binding protein (Tbp) and actin gamma (Actg). Data were analyzed by an ANOVA with a Tukey post-hoc test where different letters indicate statistical significance $(\mathrm{p}<0.05)$. Abbreviations: NOD, leucine rich repeat receptor protein 3, NLRP3; absent in melanoma 2, AIM2; PYD And CARD Domain Containing, PYCARD; caspas-1, CASP1; gasdermin D, GSDMD; interleukin-1 beta, IL1B; interleukin-18, IL18. 


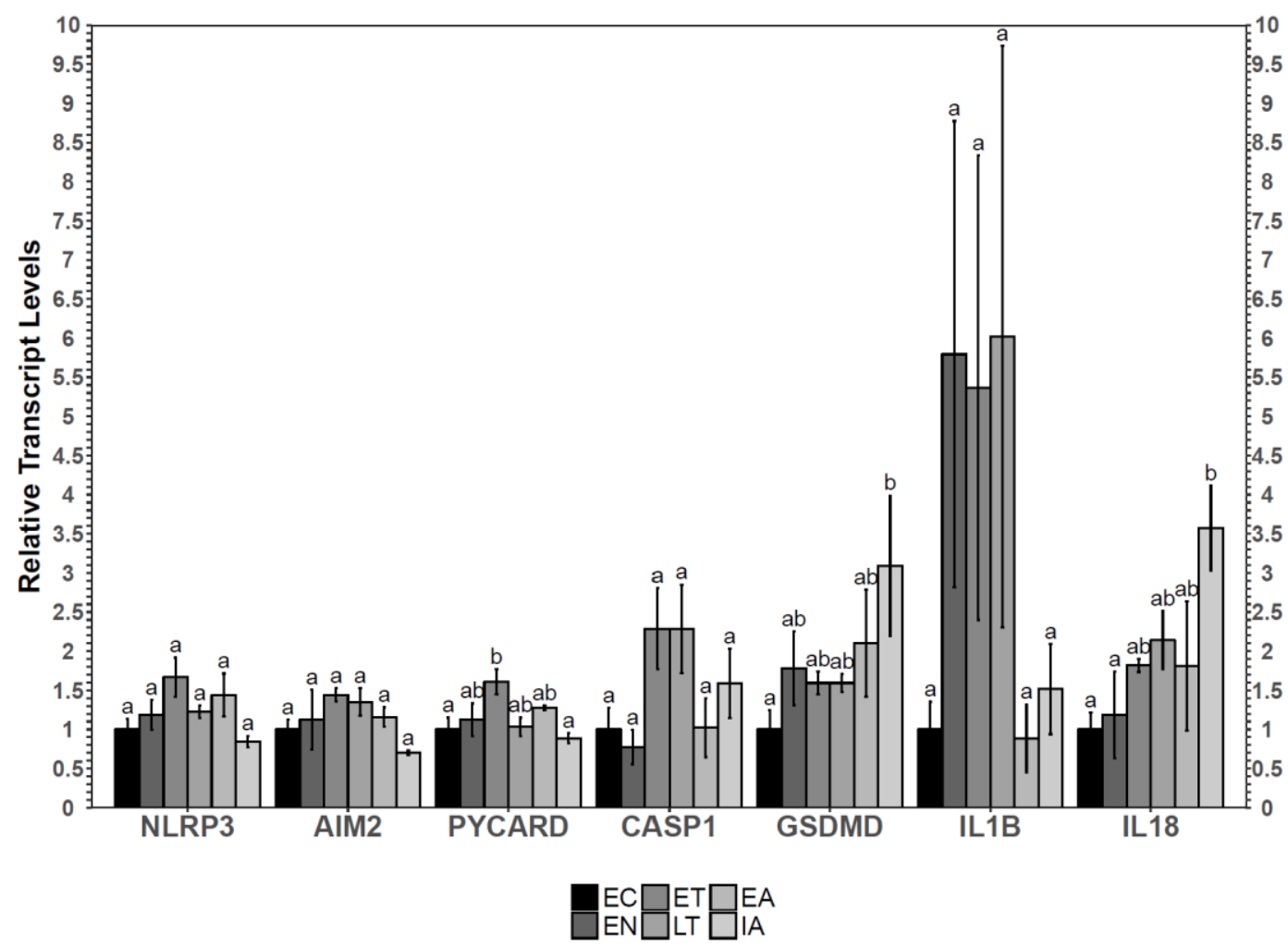

Figure 2.2: Relative transcript levels of seven key inflammasome pathway components in the white adipose tissue sampled from ground squirrels hibernating at 6 points of the torpor-arousal cycle. Actin alpha (Acta) was used as the reference gene. Data were analyzed by a one-way ANOVA with a Tukey post-hoc test where different letters indicate statistical significance $(\mathrm{p}<$ 0.05). Abbreviations and other information as in Figure 2.1. 


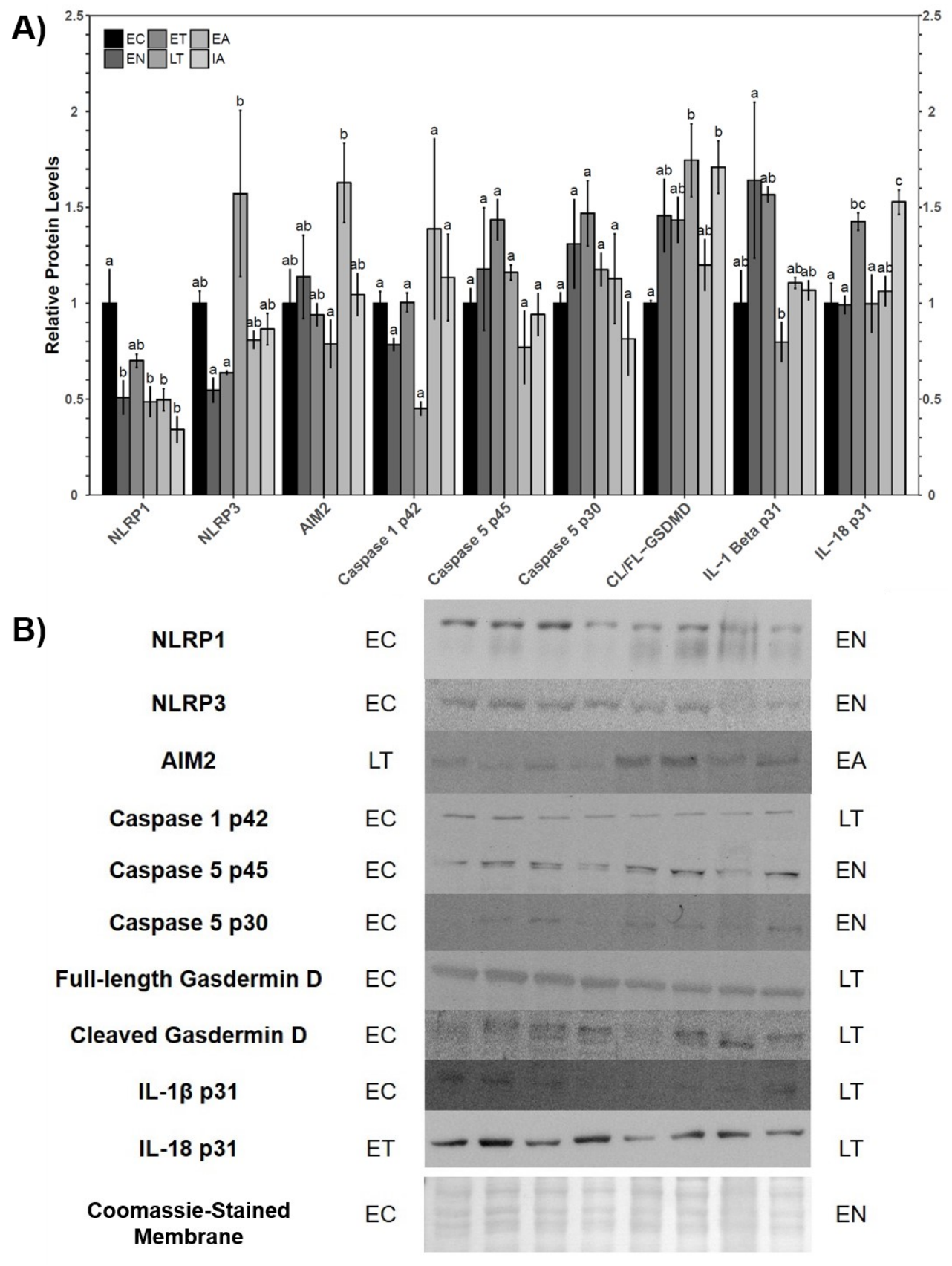

Figure 2.3: Inflammasome protein abundance patterns in BAT at 6 points of the torpor-arousal cycle. A) Histogram showing the relative protein levels, analyzed by a one-way ANOVA with a Tukey post-hoc test where different letters indicate statistical significance $(\mathrm{p}<0.05)$. B) Representative bands sampled from 4 biological replicates per time point (indicated on the left and right of the bands) for proteins whose names are in bold. Time points for representative bands were chosen to show the most notable changes between time points for each protein. Although some images are not clear, bands detected on three separate PVDF membranes confirm that western blotting was reproducible. A representative Coomassie-stained PVDF membrane shows a range of several bands in each lane ( $\mathrm{n}=4 \mathrm{EC}$ lanes followed by $\mathrm{n}=4 \mathrm{EN}$ lanes) that was used to control for even protein loading. Abbreviations: NOD, leucine rich repeat receptor protein NLRP1; CL; full-length, FL. Refer to other abbreviations in Figure 2.1. 


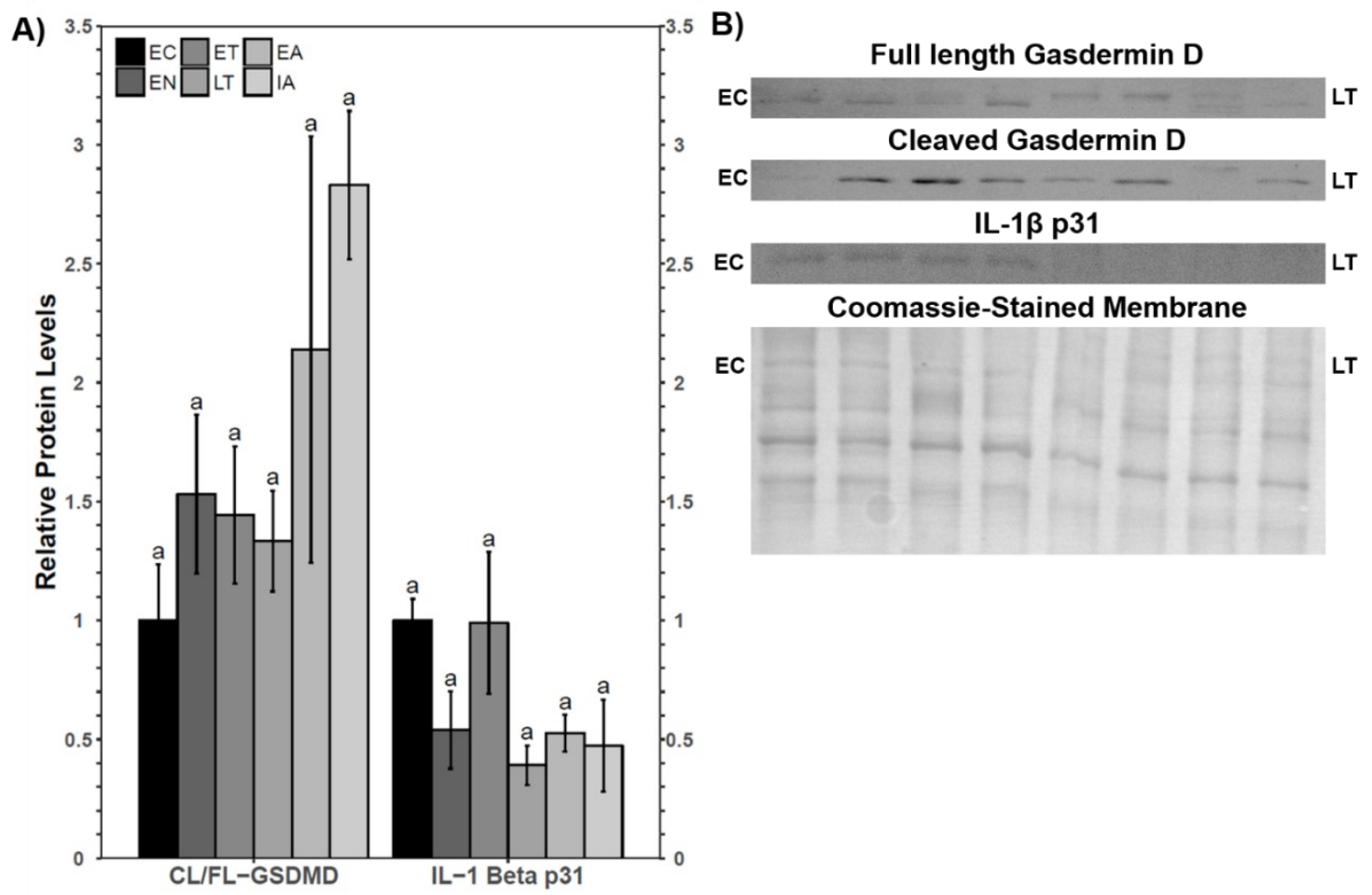

Figure 2.4: Inflammasome protein abundance patterns in white adipose tissue (WAT) at 6 points of the torpor-arousal cycle. A) Histogram showing the relative protein levels, analyzed by a oneway ANOVA with a Tukey post-hoc test where different letters indicate statistical significance ( $\mathrm{p}$ $<0.05$ ). B) Representative bands sampled from 4 biological replicates per time point (indicated on the left and right of the bands) for proteins whose names are in bold. Time points for representative bands were chosen to show the most notable changes between time points for each protein. A representative Coomassie-stained PVDF membrane shows the a range of several bands in each lane $(\mathrm{n}=4 \mathrm{EC}$ followed by $\mathrm{n}=4 \mathrm{LT})$ that was used to control for even protein loading. Refer to abbreviations in Figure 2.1 and 2.3. 


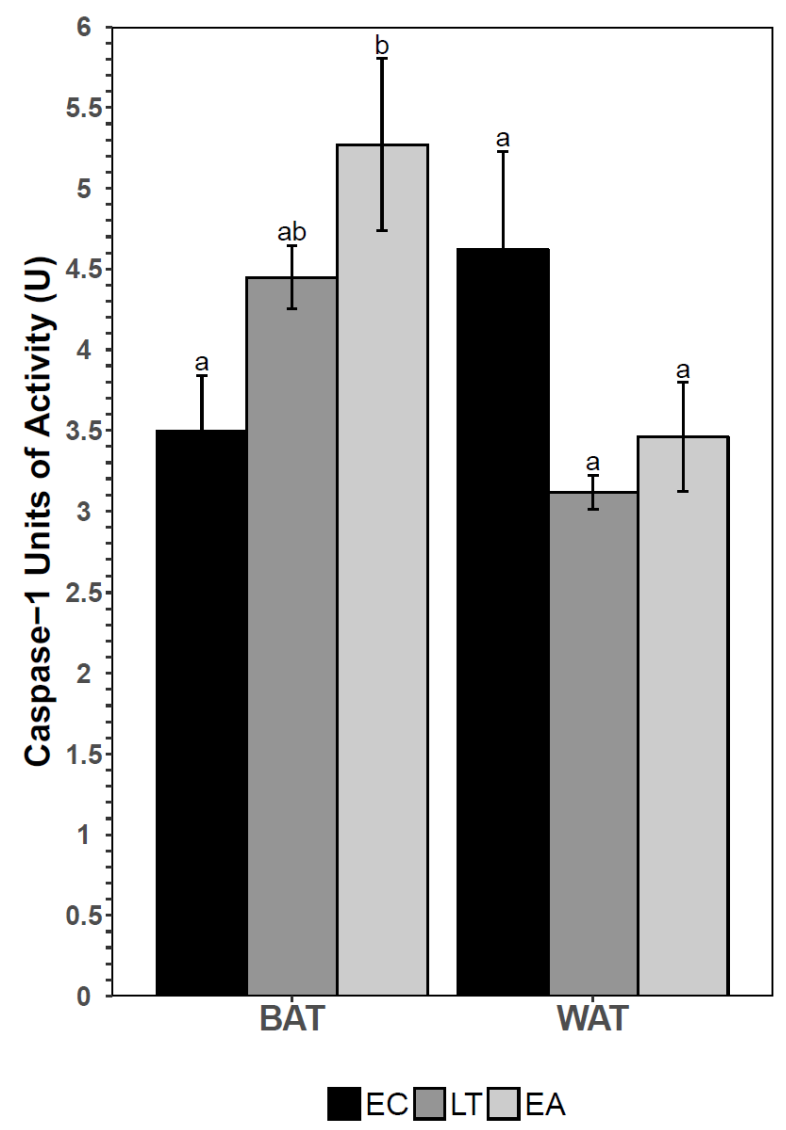

Figure 2.5: Caspase-1 activity (units) in BAT and WAT total protein homogenates from 13-lined ground squirrels sampled at three time points of the torpor-arousal cycle (EC, LT and EA). Caspase-1 activity (units), determined using a standard curve of Active recombinant Human caspase-1 (BioVision, \#1081-25), where activity was measured as absorbance at $405 \mathrm{~nm}$ following incubation of total protein with a specific caspase-1 substrate. Data were analyzed using a one-way ANOVA with a Tukey's post-hoc test. Different letters indicate statistical significance $(p<0.05)$. 


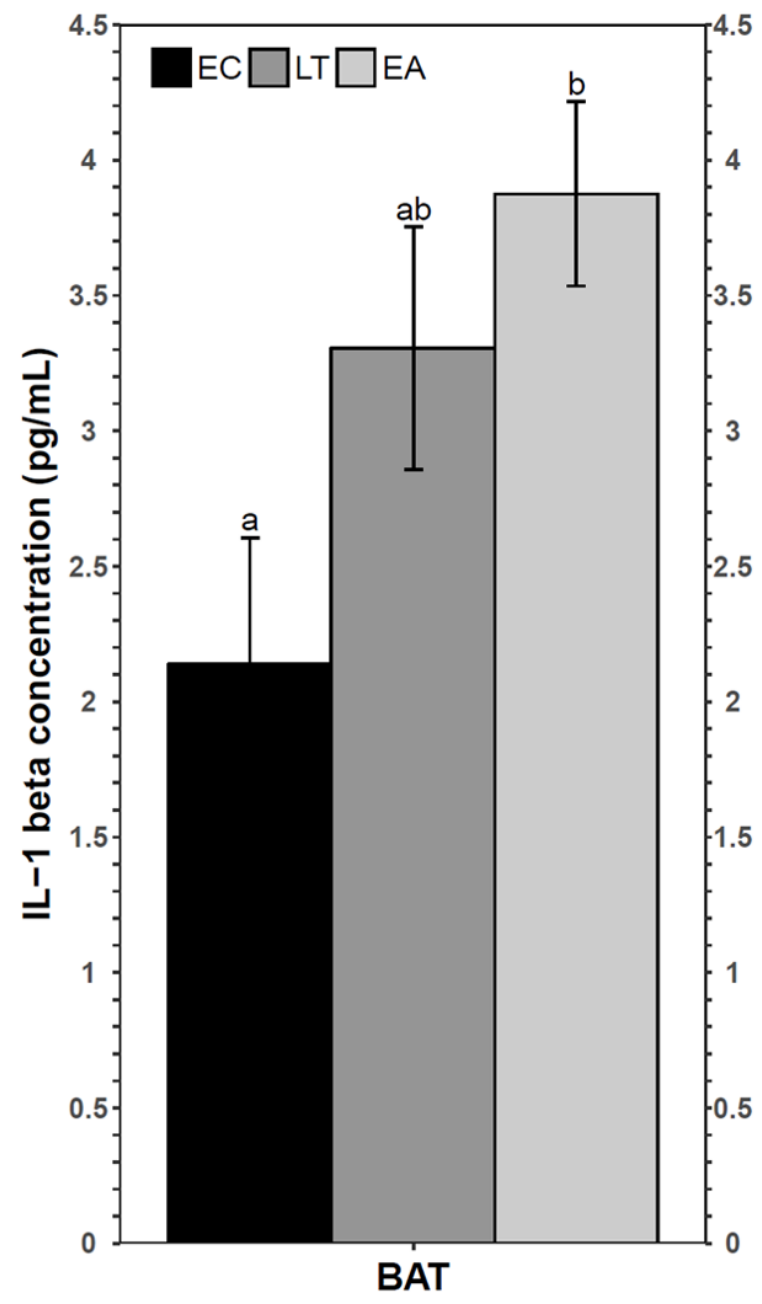

Figure 2.6: Interleukin-1 $\beta$ (IL-1 $\beta)$ concentration $(\mathrm{pg} / \mathrm{mL})$ in BAT and WAT total protein homogenates from 13-lined ground squirrels sampled at three time points of the torpor-arousal cycle (EC, LT and EA). Total IL-1 $\beta$ concentration $(\mathrm{pg} / \mathrm{mL})$ was determined by measuring absorbance values at $450 \mathrm{~nm}$ following the incubation of standards and samples with IL-1 $\beta$ specific antibodies and comparing the absorbance values to a standard curve made with mouse IL-1 $\beta$ recombinant protein. Data for both assays were analyzed using a one-way ANOVA with a Tukey's post-hoc test. Different letters indicate statistical significance $(\mathrm{p}<0.05)$. 


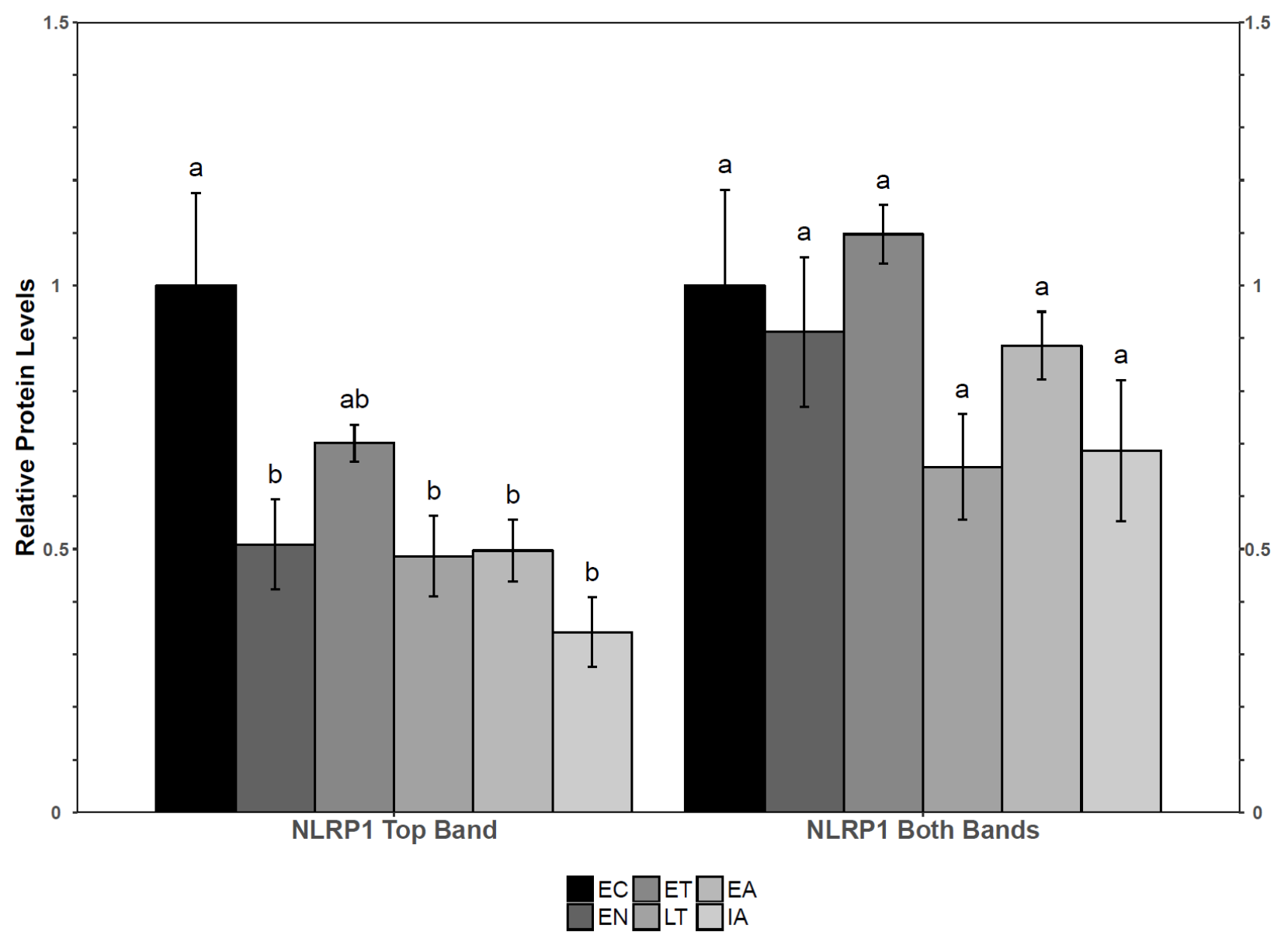

Supplemental Figure 2.1: Quantification of NLRP1 in BAT where A) the top band is quantified on its own and B) both bands are quantified together. Histograms show relative protein levels, analyzed by a one-way ANOVA with a Tukey post-hoc test where different letters indicate statistical significance $(\mathrm{p}<0.05)$. Refer to abbreviations in Figure 2.1. 
3 Markers of tissue remodeling and inflammation in the white and brown adipose tissues of a model hibernator 


\subsection{Abstract}

The 13-lined ground squirrel is a model fat-storing hibernator that nearly doubles its weight in the fall to fuel metabolism with triglycerides throughout the winter months. Hibernator brown and white adipose tissue (BAT, WAT) are important to study in terms of their inflammatory profile and tissue remodeling mechanisms since controlled and natural regulation of these processes could inform new pharmacological interventions that limit oxidative stress and inflammation in the adipose tissues of humans suffering from obesity, promote non-shivering thermogenesis-mediated weight loss, or prevent tissue damage in transplantable organs emerging from cold-storage. Thus, markers of inflammation like cytokines and soluble receptors and tissue remodeling proteins such as matrix metalloproteinases (MMPs) and tissue inhibitors of metalloproteinases (TIMPs) were investigated in normothermic, torpid, and arousing ground squirrels. Multiplex protein assays and western blotting revealed fewer changes in WAT compared to BAT. Pro-inflammatory IL-1 $\alpha$ levels increased during torpor and soluble epidermal growth factor receptor protein levels increased during arousal in BAT. Given their known roles in other model systems, these proteins could regulate processes like adipogenesis, lipid catabolism, or cell motility. Decreased TIMP2 levels combined with maintained MMP2 or MMP3 protein levels suggested that BAT may avoid tissue remodeling until arousal. No changes in WAT inflammatory cytokines or soluble receptors as well as decreased MMP2 levels during torpor and arousal suggested inflammation and modification to the extracellular matrix is likely suppressed in WAT. This study emphasizes the fat-but-fit nature of the hibernating ground squirrel and the ability of its fat stores to suppress inflammation.

Keywords: Torpor, 13-lined ground squirrel (Ictidomys tridecemlineatus), cytokine, IL-1 $\alpha$, matrix metalloproteinases, sEGFR 


\subsection{Introduction}

Seasonal, fat-storing hibernators are an important model of natural obesity that can be used to determine how "fat-but-fit" mammals control their metabolism and ward off secondary diseases associated with chronic overweight or obesity. In the fall, hibernating ground squirrels, the hibernating species of interest in this study, amass nearly two-times their summer body weight in adipose tissue, in preparation for approximately 7 months of hibernation (Storey, 2010). Like humans with obesity, autumn hibernators can become insulin and leptin resistant, perhaps to facilitate fat accumulation given that both hormones can induce satiety (Doherty et al., 2014; Florant et al., 2004; Jastroch et al., 2016; Rigano et al., 2017). Remarkably, unlike humans, hibernators reverse their hyperinsulinemia and insulin resistance at the onset of torpor to encourage lipolysis and prevent adipose dysfunction (Buck et al., 2002; Doherty et al., 2014). More specifically, 13-lined ground squirrel serum insulin levels are increased during the fall hyperphagic period but return to summer euthermic levels early in both torpid and interbout aroused ground squirrels (Buck et al., 2002). By contrast, hibernators may remain leptin resistant well into the hibernation season, where marmots only decrease leptin levels to summer levels in February (Florant et al., 2004). These are just two examples of ways hibernators dynamically and precisely control their metabolism. As they transition into a state of torpor, body temperature $\left(\mathrm{T}_{\mathrm{b}}\right)$ decreases in a controlled manner to as low as $-2.9{ }^{\circ} \mathrm{C}$ (in Arctic ground squirrels) and metabolic rate decreases to $1-5 \%$ of the euthermic metabolic rate (Geiser, 2004; Storey, 2010). During torpor, which can last up to 2 weeks, they are efficient machines: they metabolize tightly packed fatty acids stored in white adipose tissue (WAT) as ketone bodies instead of carbohydrates as fuel and they shut down all but the most important molecular pathways to reduce ATP consumption. Arousal from a torpor bout involves a return to euthermic metabolic rate and body temperature, which is facilitated by non-shivering thermogenesis in brown adipose tissue (BAT). These 
arousals occur 12-20 times per hibernation season and may be important for cellular waste removal or restful sleep.

Naturally, it would be expected that chronic obesity could be stressful for a mammalian hibernator, even if it only lasts 6-9 months of the year. Additional stressors such as changes in cell membrane fluidity, ion flux, and the generation of reactive oxygen species may occur during periodic transitions into and out of torpor. These changes could be damaging to cells, causing cell death and the production of inflammatory cytokines. Cytokines can control the recruitment of immune cells to sites of damage or pathogenic insult (Figure 3.1). Indeed, circulating immune cells as well as erythrocytes, platelets, and clotting factors are significantly decreased during torpor but resume circulation during arousal, and it is still incompletely unknown where these cells and proteins may be sequestered (Cooper et al., 2016; Lechler and Penick, 1963; Pivorun and Sinnamon, 1981). Therefore, cytokines, matrix metalloproteinases (MMPs) and their antagonists, soluble cytokine receptors and tissue inhibitors of metalloproteinases (TIMPs) were measured in WAT and BAT from a model hibernator, the 13-lined ground squirrel (Ictidomys tridecemlineatus), to determine if torpor and early arousal time points of the torpor-arousal cycle may be associated with inflammation or tissue remodeling. Importantly, some degree of tissue remodeling must occur to permit immune cell infiltration, to heal damage caused by physical stress or cell death, and to promote angiogenesis and adipogenesis (Choe et al., 2016).

MMPs are enzymes involved in tissue remodeling, given that they can cleave a variety of structural proteins such as elastin, collagen, fibronectin, laminin, etc. (Figure 3.2). MMPs help restructure the extracellular matrix and encourage angiogenesis and adipogenesis, but they are also able to cleave non-ECM proteins like cytokines, growth factors, endothelial adhesion molecules, and receptors, suggesting they may also play a role in regulating the immune response (Levine, 2008; McQuibban et al., 2002). Experiments on the lungs of Syrian hamsters showed differences in biomarkers of tissue remodeling comparing euthermia, late torpor and early 
arousal. Specifically, nuclear factor- $\kappa \mathrm{B}(\mathrm{NF} \kappa \mathrm{B})$, and cell adhesion proteins intercellular adhesion molecule 1 (ICAM-1) and vascular cell adhesion protein 1 (VCAM-1) were more abundant during torpor, and enzyme activities of angiotensin-1 converting enzyme and gelatinases (such as MMP-2 and MMP-9) decreased during torpor (Talaei et al., 2012, 2011). Similar to extracellular matrix degradation in hamster lung, the immune response is largely inhibited during torpor, but may resume activity during interbout arousals to eliminate microbial threats, clear up metabolic waste, and facilitate the repair of endogenous tissue damage (Bohr et al., 2014; Prendergast et al., 2002). Pro-inflammatory cytokines such as tumor necrosis factor alpha (TNF $\alpha)$ and interleukin-1 beta (IL-1 $\beta$ ) can upregulate MMPs to facilitate tissue remodeling, as they do in models of obesity-associated co-morbidities including osteoarthritis and cardiovascular disease (Berg et al., 2014; Wang and He, 2018).

WAT and BAT have endocrine functions since their adipocytes and tissue resident immune cells produce cytokines (adipokines) and growth factors that if released into circulation, can modulate whole-body homeostasis. Adipokines that are produced by adipose tissue can regulate inflammation, apoptosis, and energy metabolism by upregulating specific downstream cytokines and other proteins in local and distal tissues. Pro-inflammatory adipokines including leptin, IL-6, TNF $\alpha$, and IL-1 are often found in higher levels in the serum of humans suffering from obesity, relative to non-obese human controls (Azizian et al., 2016; Wang and He, 2018). By contrast, adiponectin is often regarded as an anti-inflammatory adipokine that prevents excessive inflammation and is lower in individuals with obesity relative to non-obese controls. Cytokines and growth factors are important to measure in the adipose tissues of hibernators because they regulate a range of processes, including energy metabolism, tissue remodeling, and the immune and inflammatory responses to damage markers. Soluble receptors can function as decoy receptors that have similar binding affinity for cytokines as their membrane-bound counterparts, but they are unable to invoke downstream cellular signaling (Jones and Rose-John, 
2002). To date, only a handful of studies have investigated cytokines or tissue remodeling proteins in tissues from mammalian hibernators (Cogut et al., 2017; Kurtz et al., 2006; Logan and Storey, 2019; Talaei et al., 2012, 2011; Tessier et al., 2015), and none have focused on WAT or BAT despite these tissues having important endocrine functions. The current study aims to determine if a model obligate hibernator such as the 13-lined ground squirrel regulates inflammation and tissue remodeling, comparing control animals to those in deep torpor and those emerging from metabolic suppression. Euthermic in the cold room, deep torpor and early arousal were chosen because recent studies indicate there are differences in inflammatory signaling and tissue remodelling profiles at these time points in other hibernator species and tissues (Allan and Storey, 2012; Cogut et al., 2017; Logan and Storey, 2021, 2018; Talaei et al., 2012, 2011).

\subsection{Methods}

\subsubsection{Animals}

Male 13-lined ground squirrels (Ictidomys tridecemlineatus) were wild-captured by a United States Department of Agriculture licensed trapper (TLS Research, Bloomingdale, IL) before being transported to the National Institutes of Health (Bethesda, MD, USA) for experimental treatments. The laboratory of Dr. J.M. Hallenback performed the ground squirrel hibernation experiments in accordance with guidelines set by National Institute of Neurological Disorders and Stroke (NINDS) animal care and use committee (ACUC, \#ASP 1223-05). Ground squirrels had been fed ad libitum until they gained sufficient weight for hibernation. Then, the animals were transferred to dark environmental chambers at $5{ }^{\circ} \mathrm{C}$. Body temperature $\left(\mathrm{T}_{\mathrm{b}}\right)$ was monitored by a subcutaneously injected sensor chip. Time elapsed in a hypothermic state and respiration rate were used to determine sampling points. Tissue samples were collected from ground squirrels at one of three time points of the torpor-arousal cycle: euthermic in the cold room (EC), late torpor (LT) or early arousal (EA). EC ground squirrels had a stable $\mathrm{T}_{\mathrm{b}}\left(37^{\circ} \mathrm{C}\right)$ in the $5{ }^{\circ} \mathrm{C}$ chambers and were able to enter torpor but had not done so for 3 days. LT ground 
squirrels were continuously in deep torpor for at least 5 days with $T_{b}$ values of $5-8^{\circ} \mathrm{C}$. EA ground squirrels were sampled as they transitioned out of a torpor bout. Their $\mathrm{T}_{\mathrm{b}}$ values were within the range of $9-12{ }^{\circ} \mathrm{C}$ (and rising) and they had increased respiratory rate of 60 breaths/min. Tissue samples, frozen immediately in liquid nitrogen following dissection, were shipped to Carleton University on dry ice and were stored at $-80{ }^{\circ} \mathrm{C}$ until use.

\subsubsection{Protein extraction}

Protein extracts were prepared from frozen tissue samples as previously described (Rouble et al., 2014). Briefly, 100-150 mg of frozen brown adipose tissue (BAT) and white adipose tissue (WAT) from EC, LT, and EA ground squirrels was immediately homogenized 1:3 w/v (BAT) or 1:2 w/v (WAT) in ice-cold Cell Lysis buffer (EMD Millipore, \#43-040) supplemented with $1 \mathrm{mM} \mathrm{Na} 3 \mathrm{VO} 4,10 \mathrm{mM} \mathrm{NaF}, 10 \mathrm{mM} \beta$-glycerophosphate, and $10 \mu \mathrm{L} / \mathrm{mL}$ protease inhibitor (BioBasic, \#PIC001.1). About 10-15 strokes of a glass Dounce homogenizer were used to homogenize the tissue. Each sample incubated on ice for 30 min with vortexing every $10 \mathrm{~min}$. Samples were centrifuged at $8,960 \times \mathrm{g}$ for $20 \mathrm{~min}$ at $4{ }^{\circ} \mathrm{C}$ and the supernatant was collected. Protein concentration was determined using the Bradford assay (Bio-Rad, \#5000006) and samples were standardized with Assay buffer (Millipore-Sigma, \#43-041). For multiplex analysis, five biological replicates were prepared for each time point for BAT, and four biological replicates were prepared for WAT. For western blotting, the same protein extraction protocol was followed, except five biological replicates were prepared for each time point. The Bradford assay was used to determine protein concentration before samples were combined 1:1 with $2 \mathrm{X}$ sodium dodecyl sulfate (SDS) buffer (100 mM Tris base, 4\% w/v SDS, 20\% v/v glycerol. $0.2 \% \mathrm{w} / \mathrm{v}$ bromophenol blue and $10 \% \mathrm{v} / \mathrm{v}$ beta-mercaptoethanol). The WAT samples had a final concentration of $2.5 \mu \mathrm{g} / \mu \mathrm{L}$ and the BAT samples had a final concentration of $5 \mu \mathrm{g} / \mu \mathrm{L}$. The samples were boiled and stored at $-20{ }^{\circ} \mathrm{C}$ until use. 


\subsubsection{Multiplex analysis}

Multiplex panels were used to measure relative protein levels of cytokines and growth factors, soluble receptors and inhibitors of extracellular matrix remodeling proteins. Multiplex assays were used to detect a custom array of cytokines and growth factors (R\&D Systems Human Pre-mixed Multi-Analyte Magnetic Luminex Assay, \#LXSAHM-10), and TIMPs (R\&D Systems, Human TIMP Magnetic Luminex Assay, \#LKTM003). The Human Soluble Cytokine Receptor Array 14-plex (\#HDSCR-14) assay was performed by the personnel of Eve Technologies (University of Calgary), following the manufacturer's instructions. Targets for each of these panels included a) IL-10, IL-13, IL-18, IL-1a, IL-Ira, IL-4, IL-6, MCP-1/CCL2, TNFa, VEGF-A, MCP-3/CCL7, b) sCD30, sEGFR, sgp130, sIL-1RI, sIL-1RII, sIL-2Ra, sIL-4R, sIL-6R, sTNFRI, sTNFRII, sVEGFR1, sVEGFR2, sVEGFR3, and c) TIMP1-4.

To determine the appropriate amount of protein to load into the multiplex wells, a separate pooled sample for BAT and WAT were tested to ensure signal was above the calibrator diluent blank and that the signal was not oversaturated. The standard curve provided by Eve Technologies was also used to determine an appropriate amount of protein to load. Each sample was diluted to an appropriate concentration using each kit-specific Calibrator diluent. Each assay was performed as per the recommendations of the manufacturer, and as previously described (Logan et al., 2016). With the exception of the cytokine and growth factor multiplex where the samples incubated with the diluted microparticles overnight on a plate shaker, the samples incubated with the microparticles for 2 hours at room temperature on a plate shaker.

\subsubsection{Western blotting}

Immunoblotting was performed as previously described (Logan et al., 2019). Briefly, even amounts $(\sim 25 \mu \mathrm{g})$ of each protein sample were loaded into individual wells of the $10 \%$ SDS-PAGE gels. An $n=5$ biological replicates was used for each time point except EA $(n=4)$ due to a finite number of wells in the gel. Cell Signaling monoclonal primary antibodies for MMP2 (\#87809) and MMP3 (\#14351) were diluted 1:1000 v/v in TBST (50 mM Tris-HCl, pH 
$6.8,150 \mathrm{mM} \mathrm{NaCl}, 0.05 \% \mathrm{v} / \mathrm{v}$ Tween-20). HRP-linked anti-rabbit goat IgG secondary antibody (BioShop, \#APA007P.2) was diluted 1:4000-1:8000 in TBST. Bands were visualized by enhanced chemiluminescence. Coomassie Blue $(0.25 \% \mathrm{w} / \mathrm{v}$ Coomassie brilliant blue, $7.5 \% \mathrm{v} / \mathrm{v}$ acetic acid, 50\% methanol) was used to stain the PVDF membranes that were used as a proteinloading control.

\subsubsection{Statistical analyses}

Luminex protein samples were standardized based on measurements of total protein (via the Bradford assay) to ensure that any observed fluctuations in specific proteins were not the result of differing total protein concentrations. Only fluorescence intensity values that were above the blank and were obtained from sufficient bead counts (a minimum of 50) were used in the analyses. Thus, $\mathrm{n}=4-5$ (BAT) or $\mathrm{n}=3-4$ (WAT) biological replicates were used to determine the relative protein levels of each analyte, except in the following circumstances. BAT vascular endothelial growth factor A (VEGF-A) was quantified with EC used $n=4$, and BAT IL-18 was quantified with LT $n=4$. For the same multiplex assay, WAT was $n=4$ for EC and LT except an $\mathrm{n}=3$ used for EC (TNF $\alpha$, IL-10, VEGF-A, IL-1Ra, and IL-18) and LT (CCL2, and IL-18). EC1 values were removed from the soluble receptors multiplex assay because they were uniformly higher across all analytes, suggesting error in the amount of loaded protein. BAT sRAGE was quantified with EC $\mathrm{n}=3$ and LT $\mathrm{n}=4$ as a result of discarding fluorescent intensity values lower than the blank. Low bead counts resulted in the removal of some samples from TIMP1-4 analyses. Thus, BAT TIMP levels were quantified with $\mathrm{EC} n=5, \mathrm{LT} n=4$, and $\mathrm{EA} n=4$. WAT TIMP levels were quantified with $\mathrm{EC} \mathrm{n}=4, \mathrm{LT} \mathrm{n}=3, \mathrm{EA} \mathrm{n}=3$. Analytes completely removed from the analyses included WAT and BAT IL-6 and IL-4, WAT IL-18, WAT sRAGE, BAT sgp130, due to low fluorescence intensity, low bead counts, or a combination thereof. Similarly, two of the four EA biological replicates failed at providing sufficient bead counts and so WAT EA was omitted from the study for the cytokine multiplex. 
A Luminex ${ }^{\circledR} 200$ instrument and xPonent software (Luminex ${ }^{\circledR}$ Corporation) were used to collect median fluorescent intensity (MFI). RBioplot was used to graph each analyte as the mean \pm SEM, $\mathrm{n}=$ 3-5 independent samples from different animals (Zhang and Storey, 2016). RBioplot was also used to analyze the standardized MFI values with a one-way ANOVA followed by Tukey's post hoc test, where $\mathrm{p}<0.05$ was used as the threshold for statistically significant differences. When the fluorescence intensity of EC and LT ground squirrel WAT protein samples was compared for the cytokine multiplex, RBioplot employed a Student's t-test to determine statistical significance $(\mathrm{p}<0.05)$.

For western blotting, chemiluminescent protein band signal was divided by a Coomassiestained region of the same lane in an area away from the quantified protein target, where the band density did not differ between control and hibernating states (Eaton et al., 2013). Data $(\mathrm{n}=4-5)$ are expressed as mean band density \pm SEM, relative to EC values. RBioplot was used graph and analyze the data with a one-way ANOVA with a Tukey post-hoc test, where $\mathrm{p}<0.05$ represents statistical significance (Zhang and Storey, 2016). BAT MMP2 appeared as two bands at the expected molecular weights and were quantified together. BAT MMP3 appeared as 3 bands at the anticipated molecular weights and were quantified together. WAT MMP2 and MMP3 appeared as single bands at the expected molecular weights. 


\subsection{Results}

\subsubsection{Cytokines and growth factors}

The levels of cytokines and growth factors in BAT and WAT were relatively unchanged comparing torpor and early arousal to control ground squirrels (
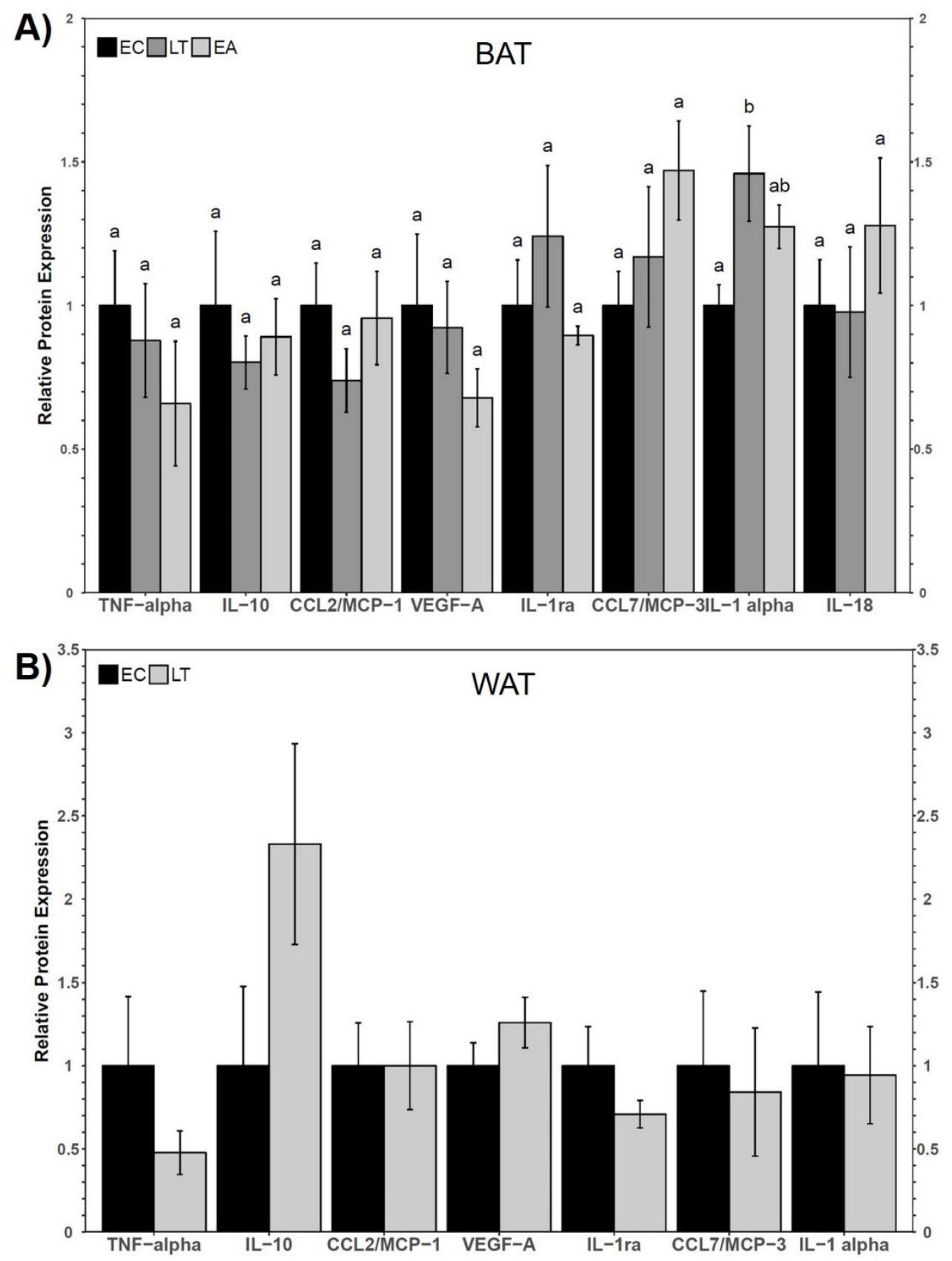

Figure 3.3). However, there was a significant increase in the levels of IL-1 $\alpha$ during torpor compared to the control. Specifically, IL-1 $\alpha$ levels increased to 1.46 -fold \pm 0.17 the control level. 


\subsubsection{Soluble receptors}

Similarly, there were no changes in the relative levels of soluble receptors in WAT,

comparing control, torpor, and early arousal (Figure 3.4). However, two soluble receptor proteins increased in BAT at the early arousal time point relative to the control group. sEGFR increased during early arousal to 1.23 -fold \pm 0.04 the control value. This change was not significant with respect to torpor.

\subsubsection{Metallopeptidase inhibitors (TIMPs)}

Due to high variation in TIMP protein abundance between biological replicates in ground squirrel WAT, there were no statistically significant changes in TIMP levels between control, torpor and early arousal (Figure 3.5). However, there was a significant decrease in TIMP2 in BAT during early arousal, where levels of TIMP2 decreased to $75 \% \pm 8 \%$ of the control level. The decrease in TIMP2 was not significant relative to the torpor condition.

\subsubsection{Matrix metalloproteinases (MMPs)}

Gelatinase A (MMP2) and Stromelysin-3 (MMP3) were quantified in WAT and BAT total protein extracts via immunoblotting. In BAT, both antibodies detected more than one band (Figure 3.6, Appendix A). The MMP2 antibody cross-reacted with two bands, one near $95 \mathrm{kDa}$ and another near $70 \mathrm{kDa}$. However, only the band at the anticipated molecular weight ( $70 \mathrm{kDa})$ was quantified. Given its position between the expected molecular weights for pro-MMP2 (72 $\mathrm{kDa})$ and active-MMP2 (68 kDa) (Wang and Khalil, 2018), this band has simply been identified as MMP2. Though MMP2 levels decreased by over 30\% relative to the euthermic control in torpor and early arousal, these changes were not significant $(\mathrm{p}=0.10$ and $\mathrm{p}=0.09$, respectively). BAT MMP3 was detected as a series of bands between $\sim 40$ and $50 \mathrm{kDa}$ which were quantified together as MMP3. By contrast, each antibody cross-reacted with a single band in WAT at the anticipated molecular weights for active-MMP2 (68 kDa) and pro-MMP3 (54 kDa) (Wang and Khalil, 2018) (Figure 3.7). There were no differences in MMP3 levels in WAT or BAT 
comparing the three time points. However, WAT MMP2 levels decreased to $54 \%$ and $41 \%$ of the euthermic level during torpor and early arousal, respectively.

\subsection{Discussion}

Inflammatory cytokines can be produced in WAT and BAT in response to cell stress such as poor angiogenesis, local hypoxia, and cell death. Cytokines can prompt local tissue inflammation, but their excretion can also affect whole-body homeostasis via binding cell-surface receptors in distal tissues. Consistent with previous studies that suggested inflammation is generally subdued during torpor (Cogut et al., 2017; Kurtz et al., 2006; Tessier et al., 2015), there were very few changes in the inflammatory profile of BAT and WAT of hibernating ground squirrels, comparing euthermic ground squirrel control group with animals sampled in late torpor 
and early arousal (
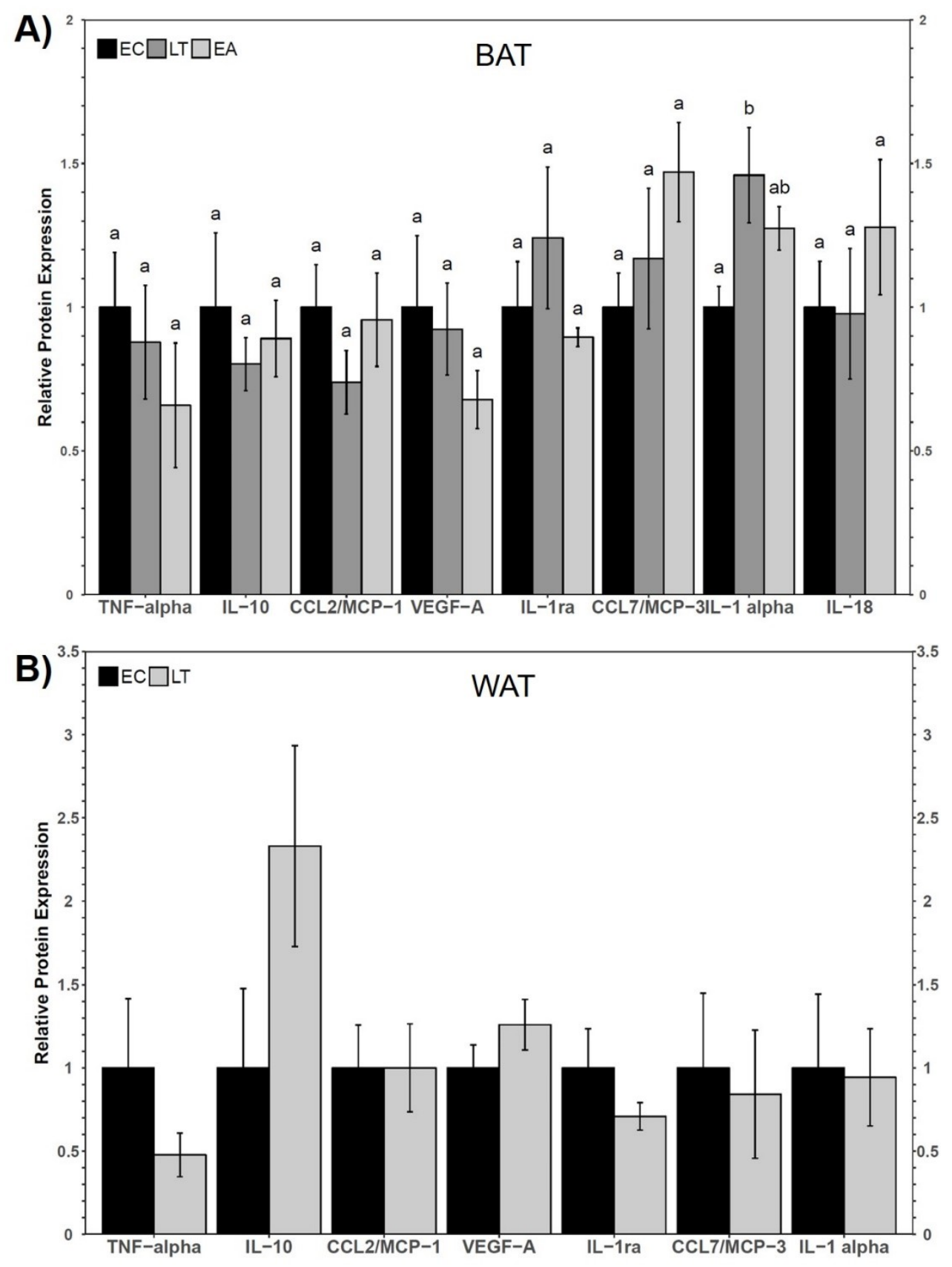

Figure 3.3). It was expected that the cytokines that are typically upregulated in humans and rodent models of obesity (e.g. TNF $\alpha$, IL-6, MCP-1, etc.) would also be upregulated in the adipose tissues of hibernators, especially during arousal when breathing and metabolic rates increase, promoting an influx of ROS. For example, hamster brain showed increased levels of pro- 
inflammatory cytokines such as IL-1 $\beta$ and IL-6 during arousal (Cogut et al., 2017). However, TNF $\alpha$, IL-6, MCP-1, and IL-18 were not upregulated in adipose tissues during deep torpor or
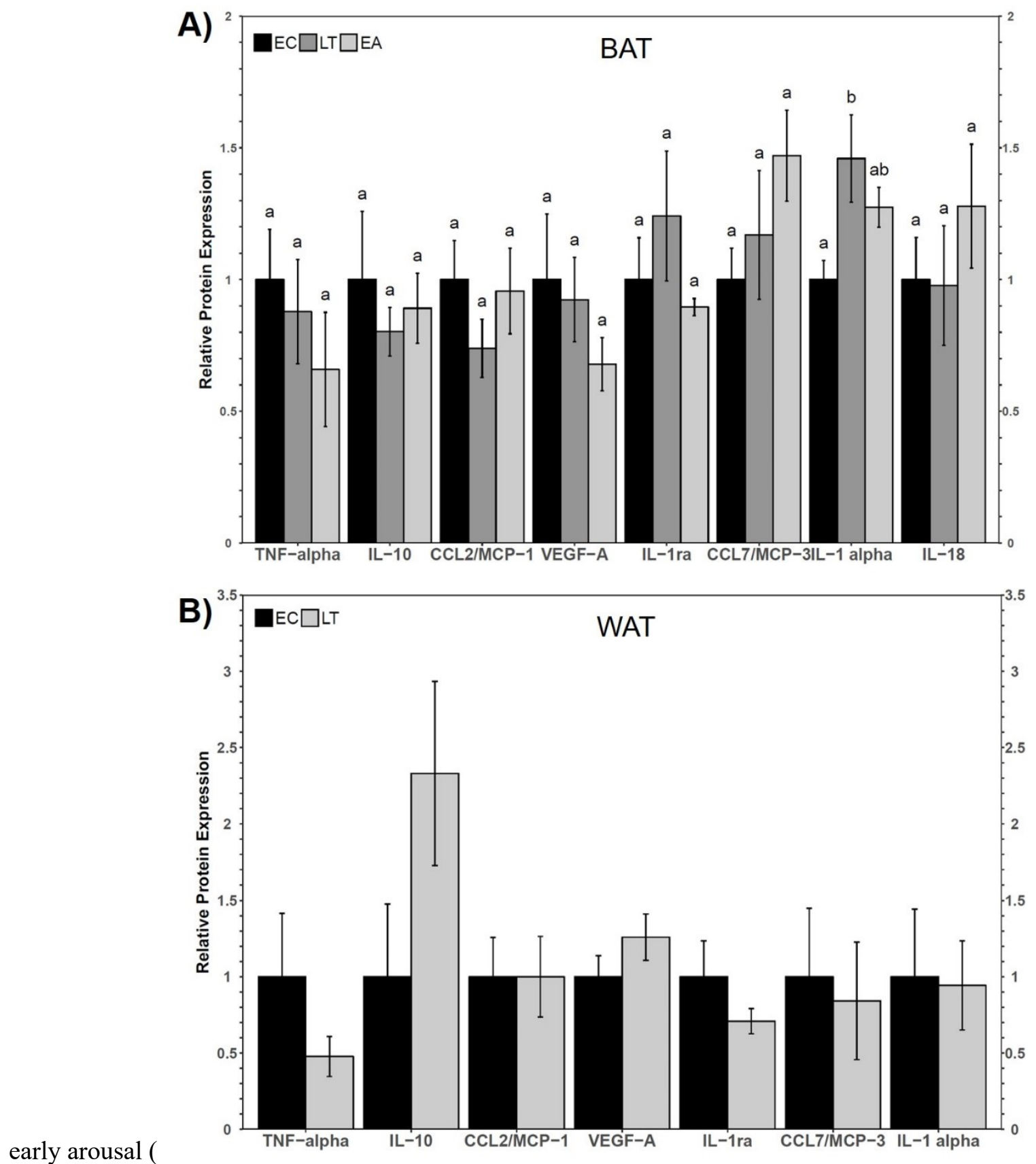

Figure 3.3), suggesting the adipose tissues of ground squirrels do not invoke inflammatory signaling at these time points of the torpor-arousal cycle. Some of the assessed cytokines (e.g. MCP-1 and MCP-3) are important chemokines that regulate the recruitment of monocytes, $\mathrm{T}$ cells 
and natural killer cells to damaged or infected tissues. These chemokines were not upregulated during torpor or arousal, so WAT and BAT are not more inflamed compared to euthermic adipose tissues. Unchanging IL-18 levels in WAT or BAT relative to the euthermic control was consistent with a recent study that showed IL-18 does not increase at the mRNA or protein levels during late torpor or early arousal in ground squirrel BAT (Logan and Storey, 2021).

Interestingly, pro-inflammatory IL-1 alpha was modestly upregulated in BAT during torpor relative to the euthermic control. Like IL-1 $\beta$, IL-1 $\alpha$ signals through IL-1R to trigger proinflammatory pathways (Figure 3.1). This is interesting because ground squirrel BAT IL-1 $\beta$ levels did not increase during LT but instead, increased during the arousal period concomitantly with caspase-1 activity (Logan and Storey, 2021). This implicates IL-1 signaling may be important throughout the torpor-arousal cycle. IL- $1 \alpha$ is different from IL-1 $\beta$ in that the pro-form of IL- $1 \alpha$ has activity, caspase- 1 is not required for IL- $1 \alpha$ activation, and gasdermin D channels are not needed to export it from the cell (Afonina et al., 2017). Therefore, IL-1 $\alpha$ upregulation during torpor could signify an inflammasome-independent mechanism of inflammatory signaling.

IL-1 $\alpha$ signaling through IL-1R receptors has been associated with increases in inflammatory cytokines and enzymes that lead to pyrogenesis (e.g. through the production of prostaglandin synthesis enzymes). Compared to murine spleen and brain, brown adipose produces higher levels of IL-1 $\alpha$, so IL-1 $\alpha$ was explored as a possible activator of heat production in coldexposed rodents (Burýšsek et al., 1993; Burýšek and Houštěk, 1996). This hypothesis was proposed because norepinephrine, the central mediator of non-shivering thermogenesis, can also upregulate the expression of pro-inflammatory cytokines including IL-1 $\alpha$ (Burýšek and Houštěk, 1996). Contrary to expectations, IL-1 $\alpha$ was not upregulated in mice exposed to $4{ }^{\circ} \mathrm{C}$ ambient temperatures to contribute to thermogenesis, and was instead downregulated by nearly $40 \%$ (Burýšsek et al., 1993). The ground squirrels in the current study were not exposed to changing ambient temperatures, but even if they were, these environmental perturbations would have little 
effect on metabolic rate (Heller and Hammel, 1972). Ground squirrels, unlike humans and mice, prevent strong BAT activation during torpor, when their core $T_{b}$ is very low. Though the trigger for BAT activation is still incompletely understood, current evidence suggests circadian rhythms and/or changes in the lowest $T_{b}$ that hibernators will defend (i.e. the thermogenic set point, $T_{\text {set }}$ ) may be important factors (McFarlane et al., 2017). BAT can be activated during torpor to keep $\mathrm{T}_{\mathrm{b}}$ above $\mathrm{T}_{\text {set, }}$ and early arousal is associated with strong activation of BAT. Since high proinflammatory cytokine levels have been associated with increases in lipolysis (Azizian et al., 2016), increases in IL-1 $\alpha$ may be important to release fatty acids from intracellular stores to facilitate low levels of non-shivering thermogenesis. However, if IL- $\alpha$ were expected to have a large role in non-shivering thermogenesis, its levels would have risen extensively during arousal 
relative to torpor and the control (
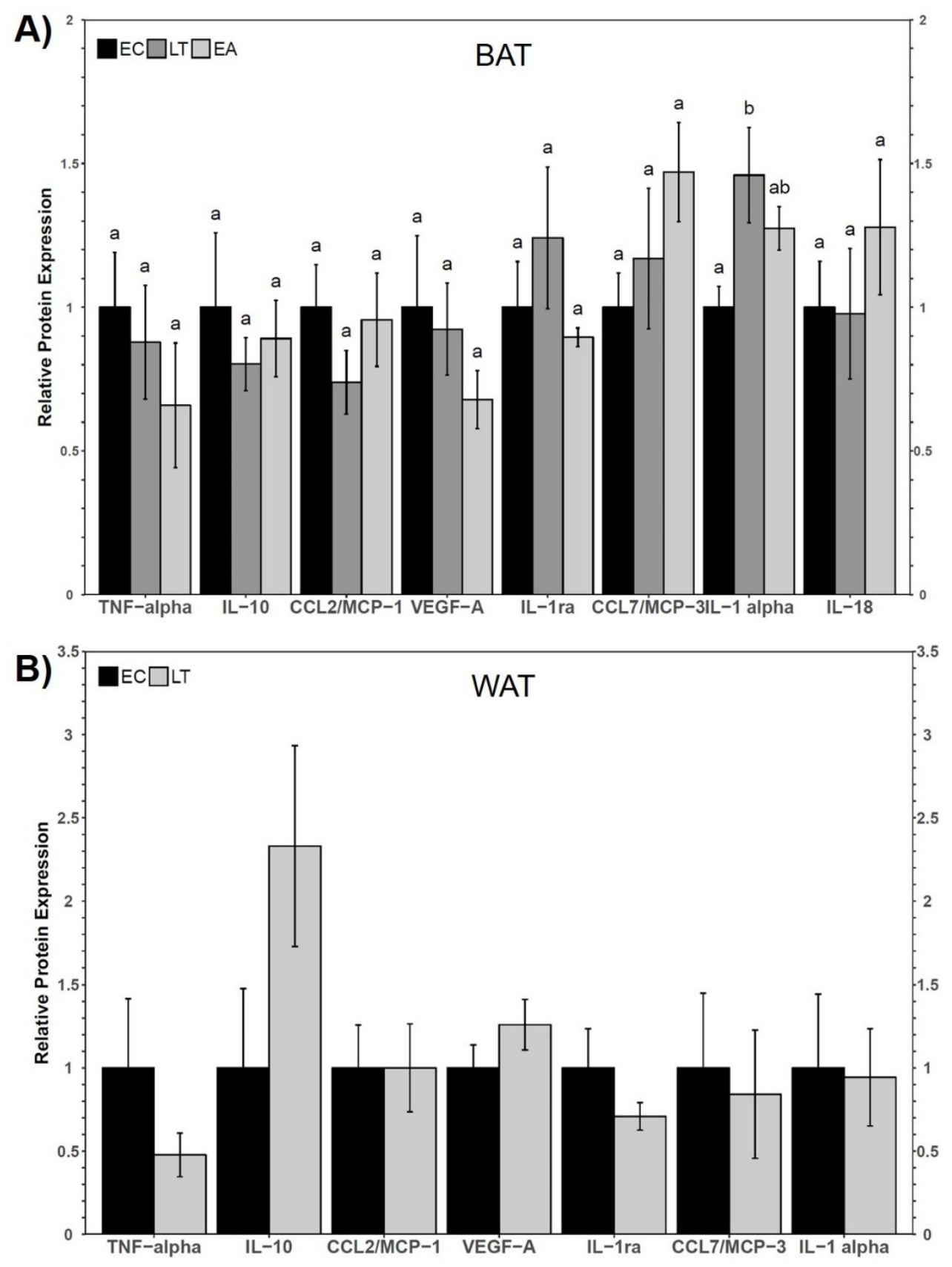

Figure 3.3). Finally, lipopolysaccharide (LPS) is a well-known inducer of IL-1 $\alpha$ expression (Burýšek and Houštěk, 1996), but its injection into hibernating ground squirrels does not increase their body temperature until the arousal period (Prendergast et al., 2002). This suggests that IL-1 $\alpha$ is not expressed during torpor as part of the host-defense mechanism but if it is, it does not induce 
thermogenesis as a result. Further experiments characterizing the molecular pathways downstream of IL- $1 \alpha$ that lead to heat production may be useful to definitively rule out a role for IL- $1 \alpha$ in pyrogenesis in hibernator BAT.

An alternative role for IL- $1 \alpha$ during torpor could be in the modulation of adipocyte differentiation, an energy expensive process that is likely inhibited during torpor, given that pathways like the cell cycle are inhibited in other hibernator tissues (Wu and Storey, 2012). Pyrogenic cytokines regulate BAT cell differentiation in mice, where IL-1 and TNF $\alpha$ were shown to prevent the differentiation of pre-adipocytes into mature adipocytes capable of non-shivering thermogenesis (Mráček et al., 2004). IL-1 $\alpha$ upregulation in pre-adipocytes is associated with decreases in peroxisome proliferator-activated receptor gamma (PPAR $\gamma$ ) and adiponectin, which are highly expressed in adipose tissues and have roles in the regulation of lipid uptake and the maturation of nascent adipocytes (Um et al., 2011). Additional experiments are needed to determine if there is a causal link between IL-1 $\alpha$ abundance and the inhibition of adipogenesis in the BAT of hibernating ground squirrels.

IL-1 $\alpha$ actions are antagonized by IL-1ra but its levels also did not change during torpor in either of the adipose tissues. In addition, the anti-inflammatory and anti-pyretic cytokine IL-10 
was not upregulated during torpor or early arousal in BAT or WAT (
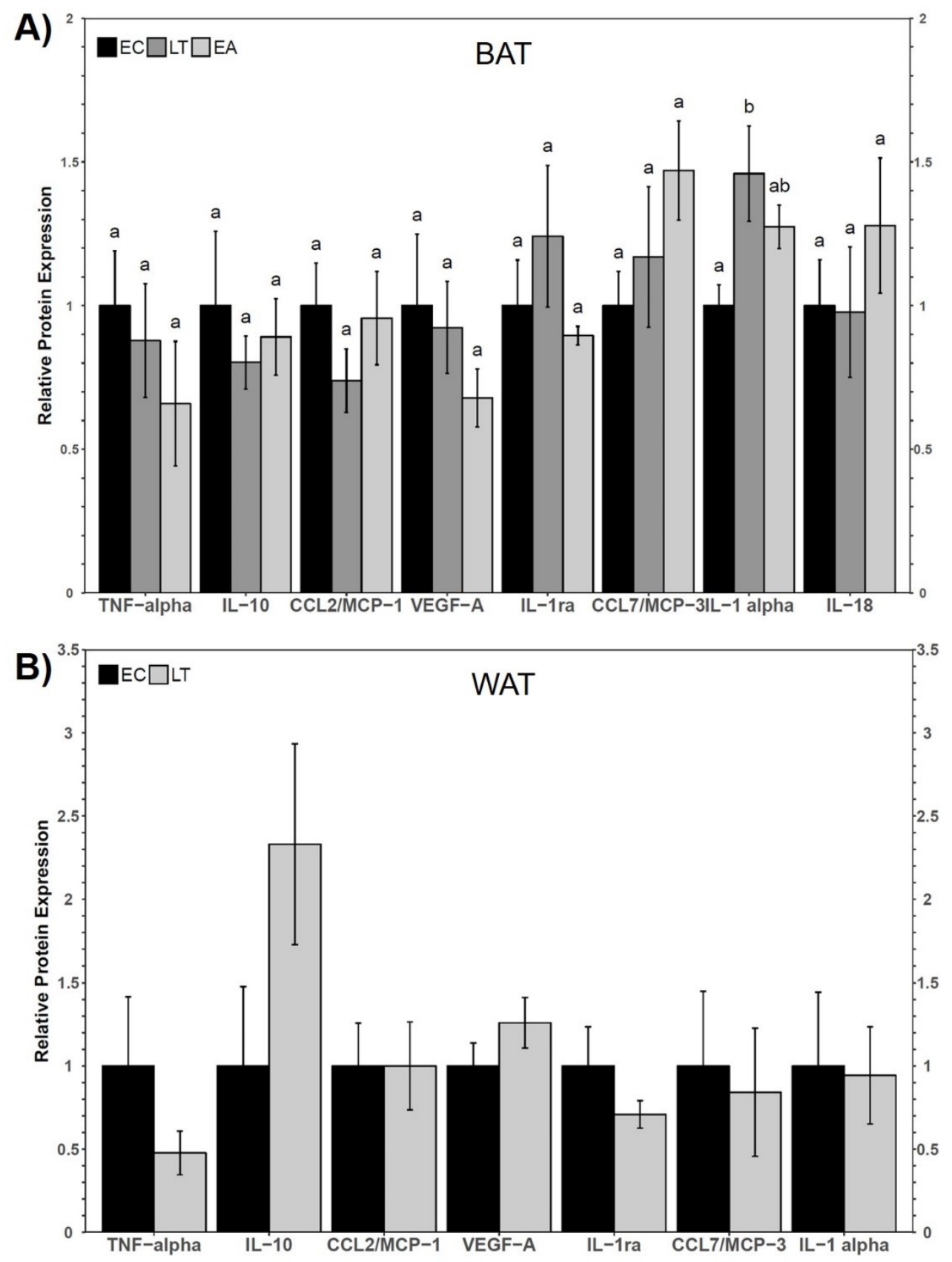

Figure 3.3). This result further suggests that inflammation does not likely occur in hibernator adipose tissues since there was no concomitant increase in anti-inflammatory markers. This is consistent with previous studies performed on hibernators. Aroused and torpid 13-lined ground squirrel lungs had lower levels of IL-10 than summer active ground squirrels (Bohr et al., 
2014), suggesting that IL-10 does not serve an anti-inflammatory role between winter euthermic, torpor, and arousal conditions. Similarly, the levels of pro- and anti-inflammatory cytokines IL-6, IL-4, IL-10, or transforming growth factor beta (TGF $\beta)$ in 13-lined ground squirrel intestine were not differentially regulated between late torpor and early arousal (Kurtz and Carey, 2007). Their levels differed with respect to a summer active control, suggesting circannual regulation of inflammatory signaling. In BAT, IL-10 receptor beta transcript levels were elevated during torpor and interbout arousal compared to both October and April euthermic controls (Hampton et al., 2013), suggesting hibernators may upregulate anti-inflammatory receptors to maintain cytokine levels high throughout hibernation. It should be noted that ground squirrel WAT showed TNF $\alpha$ levels trending towards a decrease ( $\sim 50 \%$ decrease $)$ and IL-10 levels trending towards an increase ( 2.3-fold increase) but these changes were not significant due to high variation in the levels of these cytokines in biological replicates.

Soluble receptors were investigated as potential antagonists of cytokines. Except for BAT soluble epidermal growth factor receptor (sEGFR), which increased during early arousal, there were no changes in the levels of soluble receptors in hibernating or arousing ground squirrel BAT or WAT. EGFR is a receptor tyrosine kinase and its soluble form is either produced via alternatively splicing of the mRNA or, less commonly, proteolytically cleaved by metalloproteinases (Maramotti et al., 2016; Wilken et al., 2013). Like EGFR, sEGFR binds EGF with high affinity, but lacking transmembrane and soluble domains, it serves as a decoy protein. Furthermore, sEGFR has been shown to have anti-cancer properties. This is because EGFR signaling stimulates a range of pathways including Ras, phosphoinositide 3-kinase (PI3K)-Akt, and Janus kinase-signal transducer and activator of transcription 3 (JAK-STAT3) and therefore functions in the upregulation of cell differentiation, cell motility and cytoprotection, while decreasing apoptosis (Bodnar, 2013; Maramotti et al., 2016). Thus, an increase in the sEGFR during arousal could signify active downregulation of energy expensive processes such as cell 
differentiation and angiogenesis. Indeed, in cardiac muscle from arousing 13-lined ground squirrel, phosphorylation at T654 of EGFR was increased at the early arousal time point (Childers et al., 2019). This phosphorylation site is an important marker of EGFR activity suppression that does not result in the degradation of EGFR, suggesting that hibernators use complex (but reversible) mechanisms to gate the activation of pathways downstream of EGFR. Additionally, EGFR was inspected in the lungs of hibernating hamsters due to its role in tissue remodeling during asthma and it was found that EGFR levels were lower in torpor (Talaei et al., 2011). This result was consistent with others from the same study that supported the suppression of tissue remodeling during torpor and its resumption during the arousal phase.

Tissue remodeling is a vital aspect of inflammation, but it is also needed for maintaining tissue integrity including in the accommodation of new blood vessels, expansion of lipid depots, and wound repair. Herein, the protein levels of MMP2 and MMP3 were examined because both are upregulated adipose tissues in obese animal models, and because they may be important in adipogenesis and hypertrophy, respectively (Chavey et al., 2003; Lijnen et al., 2002; Pellegrinelli et al., 2016). The expression of both MMPs is regulated by IL-1 $\beta$, IL-2, IL-6, TNF $\alpha$, and NFkB signaling (Wang and He, 2018). Interestingly, neither enzyme was upregulated in BAT (Figure 3.6), even though IL-1 $\alpha$ increased during torpor and IL-1 $\beta$ protein levels had been shown to increase during arousal (Logan and Storey, 2021). However, the results make sense given that the majority of pro-inflammatory cytokines were not increased at deep torpor or early arousal time points relative to the euthermic control. MMP2 is mainly known for its role cleaving collagen IV, but it can also cleave collagen V, collagen VII, and collagen X (Mariman and Wang, 2010). Therefore, decreased WAT MMP2 protein levels during torpor and early arousal could signify that the ECM is not remodelled during torpor or early arousal, like it may be in other hibernator tissues. 
TIMP2, an inhibitor of MMP2 and MMP3, decreased by a quarter of the control level during early arousal in WAT (Figure 3.5). In a similar study, an in vitro gelatinase activity assay showed that hamster lungs had reduced gelatinase activity during torpor (Talaei et al., 2012). Gelatinase activity was also reduced during early arousal, but not by the same extent as in deep torpor. Thus, a decrease in TIMP2 levels could facilitate the transition to arousal, when some tissue remodeling may be important. For example, the 13-lined ground squirrel lung had higher levels of E-selectin during interbout arousal, and the lung of hibernating hamster increases the amount of ICAM-1 and VCAM-1 during LT relative to arousal time points (Bohr et al., 2014; Talaei et al., 2012). These results suggest that certain hibernator tissues, such as lung, may increase pro-inflammatory cytokines that induce the expression of cell adhesion molecules, to facilitate immune cell infiltration upon arousal. Further experiments utilizing additional time points in the arousal phase may reveal similarities between adipose tissues and lung (Talaei et al., 2012, 2011).

Together, the results of this study seem to indicate the WAT and BAT do not increase the levels of many pro- or anti-inflammatory cytokines, and soluble receptors are not used as part of a major mechanism to control inflammation. MMP2 and MMP3 proteins are downregulated if not maintained, suggesting tissue remodeling is not a priority during deep torpor or early arousal in adipose tissues. Exceptions to these results include the upregulation of IL-1 $\alpha$ and sEGFR during torpor and early arousal, respectively. Both proteins may suppress energy expensive cellular processes like adipogenesis, untimely lipid oxidation, cell differentiation, or cell motility. This study suggests that BAT (but probably not WAT) from hibernating ground squirrels could increase proteins involved in inflammatory signaling during torpor and early arousal, a result that has been shown previously in terms of advanced-glycation end product (AGE) signaling and inflammasome signaling pathways (Logan and Storey, 2021, 2018). This study further emphasizes the resilience of hibernator WAT to the physiological changes that occur as 
mammalian hibernators adapt to harsh environmental conditions using metabolic rate suppression. Further experiments are warranted to characterize how fat-but fit 13-lined ground squirrels prevent WAT inflammation despite chronic obesity, and if these mechanisms are conserved across other fat-storing hibernator species. The results of these experiments could considerably advance weight loss and weight management technologies for humans. 


\subsection{References}

Afonina, I.S., Zhong, Z., Karin, M., Beyaert, R., 2017. Limiting inflammation - The negative regulation of NF-kB and the NLRP3 inflammasome. Nat. Immunol. 18, 861-869. https://doi.org/10.1038/ni.3772

Allan, M.E., Storey, K.B., 2012. Expression of NF-kB and downstream antioxidant genes in skeletal muscle of hibernating ground squirrels, Spermophilus tridecemlineatus. Cell Biochem. Funct. 30, 166-174. https://doi.org/10.1002/cbf.1832

Azizian, M., Mahdipour, E., Mirhafez, S.R., Shoeibi, S., Nematy, M., Esmaily, H., Ferns, G.A.A., Ghayour-Mobarhan, M., 2016. Cytokine profiles in overweight and obese subjects and normal weight individuals matched for age and gender. Ann. Clin. Biochem. 53, 663-668. https://doi.org/10.1177/0004563216629997

Berg, G., Schreier, L., Miksztowicz, V., 2014. Circulating and adipose tissue matrix metalloproteinases in cardiometabolic risk environments: Pathophysiological aspects. Horm. Mol. Biol. Clin. Investig. 17, 79-87. https://doi.org/10.1515/hmbci-2013-0069

Bodnar, R.J., 2013. Epidermal growth factor and epidermal growth factor receptor: the yin and yang in the treatment of cutaneous wounds and cancer. Adv. Wound Care 2, 24-29. https://doi.org/10.1089/wound.2011.0326

Bohr, M., Brooks, A.R., Kurtz, C.C., 2014. Hibernation induces immune changes in the lung of 13-lined ground squirrels (Ictidomys tridecemlineatus). Dev. Comp. Immunol. 47, 178-184. https://doi.org/10.1016/j.dci.2014.07.011

Buck, M.J., Squire, T.L., Andrews, M.T., Michael, J., Squire, T.L., Matthew, T., 2002. Coordinate expression of the PDK4 gene: a means of regulating fuel selection in a hibernating mammal. Physiol. Genomics 8, 5-13. https://doi.org/10.1152/physiolgenomics.00076.2001

Burýšek, L., Houštěk, J., 1996. Multifactorial induction of gene expression and nuclear localization of mouse interleukin 1 $\alpha$. Cytokine 8, 460-467. https://doi.org/10.1006/cyto.1996.0062

Burýšek, L., Tvrdí, P., Houštěk, J., 1993. Expression of interleukin-1 $\alpha$ and interleukin-1 receptor type I genes in murine brown adipose tissue. FEBS Lett. 334, 229-232. https://doi.org/10.1016/00145793(93)81717-E

Chavey, C., Mari, B., Monthouel, M.N., Bonnafous, S., Anglard, P., Van Obberghen, E., Tartare-Deckert, S., 2003. Matrix metalloproteinases are differentially expressed in adipose tissue during obesity and modulate adipocyte differentiation. J. Biol. Chem. 278, 11888-11896. https://doi.org/10.1074/jbc.M209196200

Childers, C.L., Tessier, S.N., Storey, K.B., 2019. The heart of a hibernator: EGFR and MAPK signaling in cardiac muscle during the hibernation of 13-lined ground squirrels, Ictidomys tridecemlineatus. PeerJ 2019, 1-20. https://doi.org/10.7717/peerj.7587

Choe, S.S., Huh, J.Y., Hwang, I.J., Kim, J.B.J.I., Kim, J.B.J.I., 2016. Adipose tissue remodeling: its role in energy metabolism and metabolic disorders. Front. Endocrinol. (Lausanne). 7, 30. https://doi.org/10.3389/fendo.2016.00030

Cogut, V., Bruintjes, J.J.J., Eggen, B.J.L.J.L., van der Zee, E.A.A., Henning, R.H.H., 2017. Brain inflammatory cytokines and microglia morphology changes throughout hibernation phases in Syrian hamster. Brain. Behav. Immun. 68, 17-22. https://doi.org/10.1016/j.bbi.2017.10.009

Cooper, S., Sell, S., Nelson, L., Hawes, J., Benrud, J.A., Kohlnhofer, B.M., Burmeister, B.R., Flood, V.H., 2016. Von Willebrand factor is reversibly decreased during torpor in 13-lined ground squirrels. J. Comp. Physiol. B Biochem. Syst. Environ. Physiol. 186, 131-139. https://doi.org/10.1007/s00360015-0941-5

Doherty, A.H., Florant, G.L., Donahue, S.W., 2014. Endocrine regulation of bone and energy metabolism in hibernating mammals. Integr. Comp. Biol. 54, 463-483. https://doi.org/10.1093/icb/icu001 
Eaton, S.L., Roche, S.L., Llavero Hurtado, M., Oldknow, K.J., Farquharson, C., Gillingwater, T.H., Wishart, T.M., 2013. Total protein analysis as a reliable loading control for quantitative fluorescent western blotting. PLoS One 8, 1-9. https://doi.org/10.1371/journal.pone.0072457

Florant, G.L., Porst, H., Peiffer, A., Hudachek, S.F., Pittman, C., Summers, S.A., Rajala, M.W., Scherer, P.E., 2004. Fat-cell mass, serum leptin and adiponectin changes during weight gain and loss in yellow-bellied marmots (Marmota flaviventris). J. Comp. Physiol. B Biochem. Syst. Environ. Physiol. 174, 633-639. https://doi.org/10.1007/s00360-004-0454-0

Geiser, F., 2004. Metabolic rate and body temperature reduction during hibernation and daily torpor. Annu. Rev. Physiol. 66, 239-74. https://doi.org/10.1146/annurev.physiol.66.032102.115105

Hampton, M., Melvin, R.G., Andrews, M.T., 2013. Transcriptomic analysis of brown adipose tissue across the physiological extremes of natural hibernation. PLoS One 8, 1-12. https://doi.org/10.1371/journal.pone.0085157

Heller, H.C., Hammel, H.T., 1972. CNS control of body temperature during hibernation. Comp. Biochem. Physiol. -- Part A Physiol. 41, 349-359. https://doi.org/10.1016/0300-9629(72)90066-7

Jastroch, M., Giroud, S., Barrett, P., Geiser, F., Heldmaier, G., Herwig, A., 2016. Seasonal control of mammalian energy balance: recent advances in the understanding of daily torpor and hibernation. J. Neuroendocrinol. 28, 1-10. https://doi.org/10.1111/jne.12437

Jones, S.A., Rose-John, S., 2002. The role of soluble receptors in cytokine biology: the agonistic properties of the sIL-6R/IL-6 complex. Biochim. Biophys. Acta Mol. Cell Res. 1592, 251-263. https://doi.org/10.1016/S0167-4889(02)00319-1

Kurtz, C.C., Carey, H. V., 2007. Seasonal changes in the intestinal immune system of hibernating ground squirrels. Dev. Comp. Immunol. 31, 415-428. https://doi.org/10.1016/j.dci.2006.07.003

Kurtz, C.C., Lindell, S.L., Mangino, M.J., Carey, H. V, 2006. Hibernation confers resistance to intestinal ischemia-reperfusion injury. Am. J. Physiol. Liver Physiol. 291, G895-G901. https://doi.org/10.1152/ajpgi.00155.2006

Lechler, E., Penick, G.D., 1963. Blood clotting defect in hibernating ground squirrels (Citellus tridecemlineatus). Am. J. Physiol. 205, 985-988. https://doi.org/10.1152/ajplegacy.1963.205.5.985

Levine, S.J., 2008. Molecular mechanisms of soluble cytokine receptor generation. J. Biol. Chem. 283, 14177-14181. https://doi.org/10.1074/jbc.R700052200

Lijnen, H.R., Maquoi, E., Demeulemeester, D., Van Hoef, B., Collen, D., 2002. Modulation of fibrinolytic and gelatinolytic activity during adipose tissue development in a mouse model of nutritionally induced obesity. Thromb. Haemost. 88, 345-353. https://doi.org/10.1055/s-0037-1613208

Logan, S.M., Luu, B.E., Storey, K.B., 2016. Turn down genes for WAT? Activation of anti-apoptosis pathways protects white adipose tissue in metabolically depressed 13-lined ground squirrels. Mol. Cell. Biochem. 416, 47-62. https://doi.org/10.1007/s11010-016-2695-0

Logan, S.M., Storey, K.B., 2021. Inflammasome signaling could be used to sense and respond to endogenous damage in brown but not white adipose tissue of a hibernating ground squirrel. Dev. Comp. Immunol. 114, 103819. https://doi.org/10.1016/j.dci.2020.103819

Logan, S.M., Storey, K.B., 2019. Angiogenic signaling in the lungs of a metabolically suppressed hibernating mammal (Ictidomys tridecemlineatus). PeerJ 2019. https://doi.org/10.7717/peerj.8116

Logan, S.M., Storey, K.B., 2018. Pro-inflammatory AGE-RAGE signaling is activated during arousal from hibernation in ground squirrel adipose. PeerJ 6, e4911. https://doi.org/10.7717/peerj.4911

Logan, S.M., Wu, C., Storey, K.B., 2019. The squirrel with the lagging eIF2: Global suppression of protein synthesis during torpor. Comp. Biochem. Physiol. Part A Mol. Integr. Physiol. 227, 161-171. https://doi.org/10.1016/j.cbpa.2018.10.014 
Maramotti, S., Paci, M., Manzotti, G., Rapicetta, C., Gugnoni, M., Galeone, C., Cesario, A., Lococo, F., 2016. Soluble epidermal growth factor receptors (sEGFRs) in cancer: biological aspects and clinical relevance. Int. J. Mol. Sci. 17, 593. https://doi.org/10.3390/ijms17040593

Mariman, E.C.M., Wang, P., 2010. Adipocyte extracellular matrix composition, dynamics and role in obesity. Cell. Mol. Life Sci. 67, 1277-1292. https://doi.org/10.1007/s00018-010-0263-4

McFarlane, S. V., Mathers, K.E., Staples, J.F., 2017. Reversible temperature-dependent differences in brown adipose tissue respiration during torpor in a mammalian hibernator. Am. J. Physiol. - Regul. Integr. Comp. Physiol. 312, R434-R442. https://doi.org/10.1152/ajpregu.00316.2016

McQuibban, G.A., Gong, J.H., Wong, J.P., Wallace, J.L., Clark-Lewis, I., Overall, C.M., 2002. Matrix metalloproteinase processing of monocyte chemoattractant proteins generates $\mathrm{CC}$ chemokine receptor antagonists with anti-inflammatory properties in vivo. Blood 100, 1160-1167. https://doi.org/10.1182/blood.v100.4.1160.h81602001160_1160_1167

Mráček, T., Cannon, B., Houštěk, J., 2004. IL-1 and LPS but not IL-6 inhibit differentiation and downregulate PPAR gamma in brown adipocytes. Cytokine 26, 9-15. https://doi.org/10.1016/j.cyto.2003.12.001

Pellegrinelli, V., Carobbio, S., Vidal-Puig, A., 2016. Adipose tissue plasticity: how fat depots respond differently to pathophysiological cues. Diabetologia 59, 1075-1088. https://doi.org/10.1007/s00125016-3933-4

Pivorun, E.B., Sinnamon, W.B., 1981. Blood coagulation studies in normothermic, hibernating, and aroused Spermophilus franklini. Cryobiology 18, 515-520. https://doi.org/10.1016/00112240(81)90212-1

Prendergast, B.J., Freeman, D.A., Zucker, I., Nelson, R.J., 2002. Periodic arousal from hibernation is necessary for initiation of immune responses in ground squirrels. Am. J. Physiol. - Regul. Integr. Comp. Physiol. 282, 1054-1062. https://doi.org/10.1152/ajpregu.00562.2001

Rigano, K.S., Gehring, J.L., Evans Hutzenbiler, B.D., Chen, A. V., Nelson, O.L., Vella, C.A., Robbins, C.T., Jansen, H.T., 2017. Life in the fat lane: seasonal regulation of insulin sensitivity, food intake, and adipose biology in brown bears. J. Comp. Physiol. B 187, 649-676. https://doi.org/10.1007/s00360-016-1050-9

Rouble, A.N., Tessier, S.N., Storey, K.B., 2014. Characterization of adipocyte stress response pathways during hibernation in 13-lined ground squirrels. Mol. Cell. Biochem. 393, 271-282. https://doi.org/10.1007/s11010-014-2070-y

Storey, K.B., 2010. Out cold: biochemical regulation of mammalian hibernation - A mini-review. Gerontology 56, 220-230. https://doi.org/10.1159/000228829

Talaei, F., Bouma, H.R., Hylkema, M.N., Strijkstra, A.M., Boerema, A.S., Schmidt, M., Henning, R.H., 2012. The role of endogenous $\mathrm{H} 2 \mathrm{~S}$ formation in reversible remodeling of lung tissue during hibernation in the Syrian hamster. J. Exp. Biol. 215, 2912-2919. https://doi.org/10.1242/jeb.067363

Talaei, F., Hylkema, M.N., Bouma, H.R., Boerema, A.S., Strijkstra, A.M., Henning, R.H., Schmidt, M., 2011. Reversible remodeling of lung tissue during hibernation in the Syrian hamster. J. Exp. Biol. 214, 1276-1282. https://doi.org/10.1242/jeb.052704

Tessier, S.N., Katzenback, B. a., Pifferi, F., Perret, M., Storey, K.B., 2015. Cytokine and antioxidant regulation in the intestine of the gray mouse lemur (Microcebus murinus) during torpor. Genomics, Proteomics Bioinforma. 13, 127-135. https://doi.org/10.1016/j.gpb.2015.03.005

Um, J.-Y., Rim, H.-K., Kim, S.-J., Kim, H.-L., Hong, S.-H., 2011. Functional polymorphism of IL-1 alpha and its potential role in obesity in humans and mice. PLoS One 6, e29524.

https://doi.org/10.1371/journal.pone.0029524 
Wang, T., He, C., 2018. Pro-inflammatory cytokines: The link between obesity and osteoarthritis. Cytokine Growth Factor Rev. 44, 38-50. https://doi.org/10.1016/j.cytogfr.2018.10.002

Wang, X., Khalil, R.A., 2018. Matrix metalloproteinases, vascular remodeling, and vascular disease, in: Physiology \& Behavior. pp. 241-330. https://doi.org/10.1016/bs.apha.2017.08.002

Wilken, J.A., Perez-Torres, M., Nieves-Alicea, R., Cora, E.M., Christensen, T.A., Baron, A.T., Maihle, N.J., 2013. Shedding of soluble epidermal growth factor receptor (sEGFR) is mediated by a metalloprotease/fibronectin/integrin axis and inhibited by cetuximab. Biochemistry 52, 4531-4540. https://doi.org/10.1021/bi400437d

Wu, C.-W., Storey, K.B., 2012. Pattern of cellular quiescence over the hibernation cycle in liver of 13-lined ground squirrels. Cell Cycle 11, 1714-1726. https://doi.org/10.4161/cc.19799

Zhang, J., Storey, K.B., 2016. RBioplot: an easy-to-use R pipeline for automated statistical analysis and data visualization in molecular biology and biochemistry. PeerJ 4, e2436.

https://doi.org/10.7717/peerj.2436

\subsection{Figures}

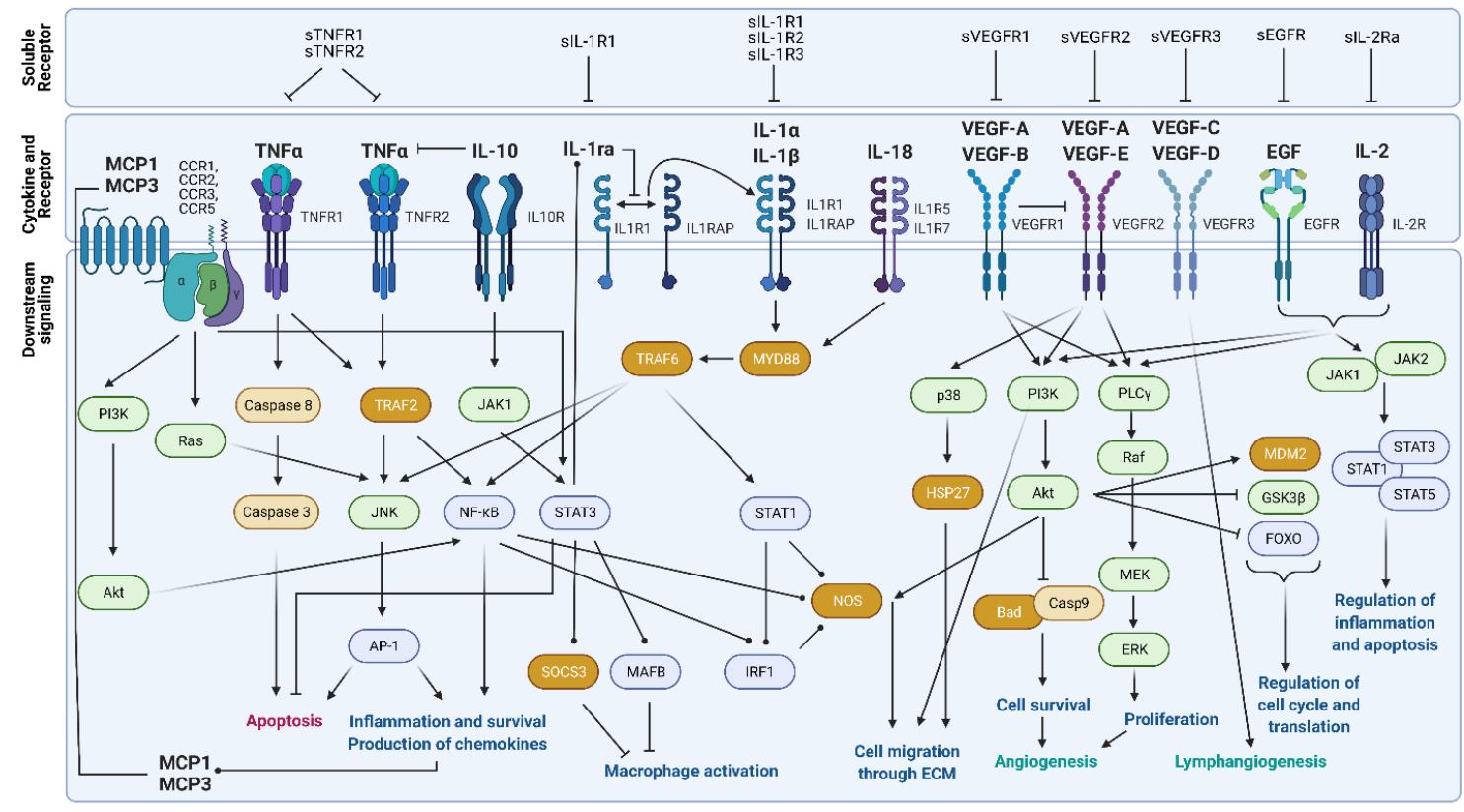

Figure 3.1: General signal transduction pathways initiated by selected cytokines (in bold) and their receptors. Cytokines bind receptors and trigger signaling cascades that activate kinases (in green), proteases (in yellow), transcription factors (in blue) and other proteins (in dark yellow). Gene expression is denoted as a line ending in a dot and repression is denoted as a line ending in a blunt end. This figure was created using BioRender. Abbreviations: protein kinase B (Akt), activator protein 1 (AP-1), BCL2 associated agonist of cell death (Bad), extracellular signal-regulated kinase (ERK), forkhead box transcription factor (FOXO), glycogen synthase kinase 3 beta (GSK3 $\beta$ ), heat shock protein (HSP), interferon regulatory factor (IRF), Janus kinase (JAK), c-Jun N-terminal kinases (JNK), MAF BZIP Transcription factor B (MAFB), mouse double minute 2 homolog (MDM2), mitogen-activated protein kinase kinase (MEK), myeloid differentiation primary response protein (MYD88), nuclear factor kappa-light-chain-enhancer of activated B cells (NFKB), nitric oxide synthase (NOS), phosphoinositide 3-kinase (PI3K), phospholipase C gamma (PLC $\gamma$ ), proto-oncogene serine/threonine-protein kinase (Raf), rat sarcoma viral oncoprotein (Ras), suppressor of cytokine signaling 3 (SOCS3), signal transducer and activator of transcription (STAT), TNF receptor associated factor (TRAF) 


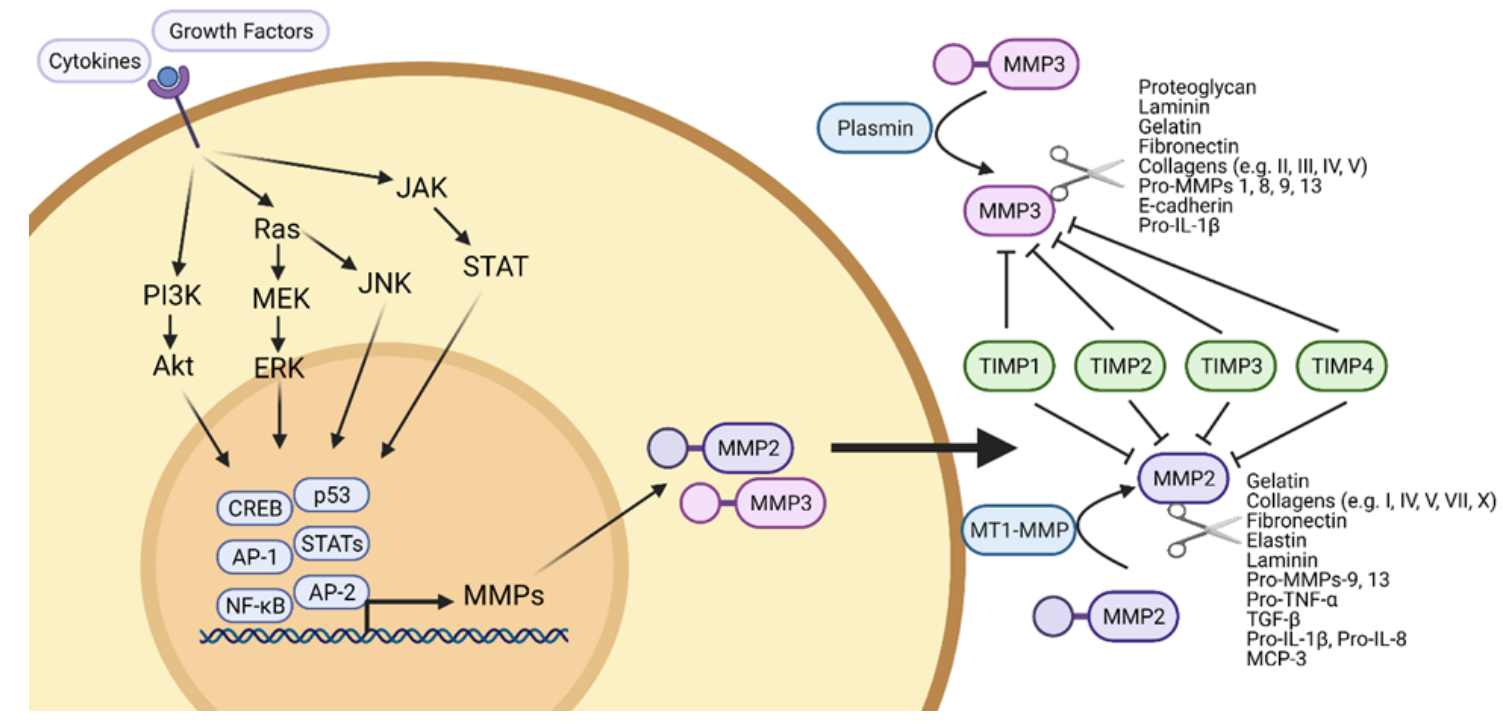

Figure 3.2: Matrix metalloproteinase (MMP) activation and substrates. Cytokines and growth factors binding to their receptors initiates kinase-dependent signaling cascades and the activation of transcription factors. Activator 2 (AP-2) and other transcription factors regulate MMP2 gene expression whereas AP-1 (c-Fos and c-Jun) and NFKB transcription factors regulate MMP3 gene expression. MMPs are expressed as pre-pro-enzymes that lose their secretion signal when they are secreted. Pro-forms are then cleaved by serine proteases such as plasmin or membrane type 1 MMP (MT1-MMP) to generate active MMP enzymes. MMP2 and MMP3 can cleave proteins that form the ECM in adipose tissue, including collagens I-IV, laminin and fibronectin. MMP2 and MMP3 can also cleave cytokines and growth factors to regulate cytokine signaling cascades. Tissue inhibitors of metalloproteinases (TIMPs) 1-4 inhibit MMP2 and MMP3 activity by binding them in a 1:1 stoichiometric ratio. This figure was created using BioRender.

Abbreviations as in figure 3.1 and as follows: cAMP Response Element-Binding Protein (CREB), monocyte chemoattractant protein (MCP), transforming growth factor beta (TGF- $\beta$ ), tumor necrosis factor alpha $(\mathrm{TNF} \alpha)$. 

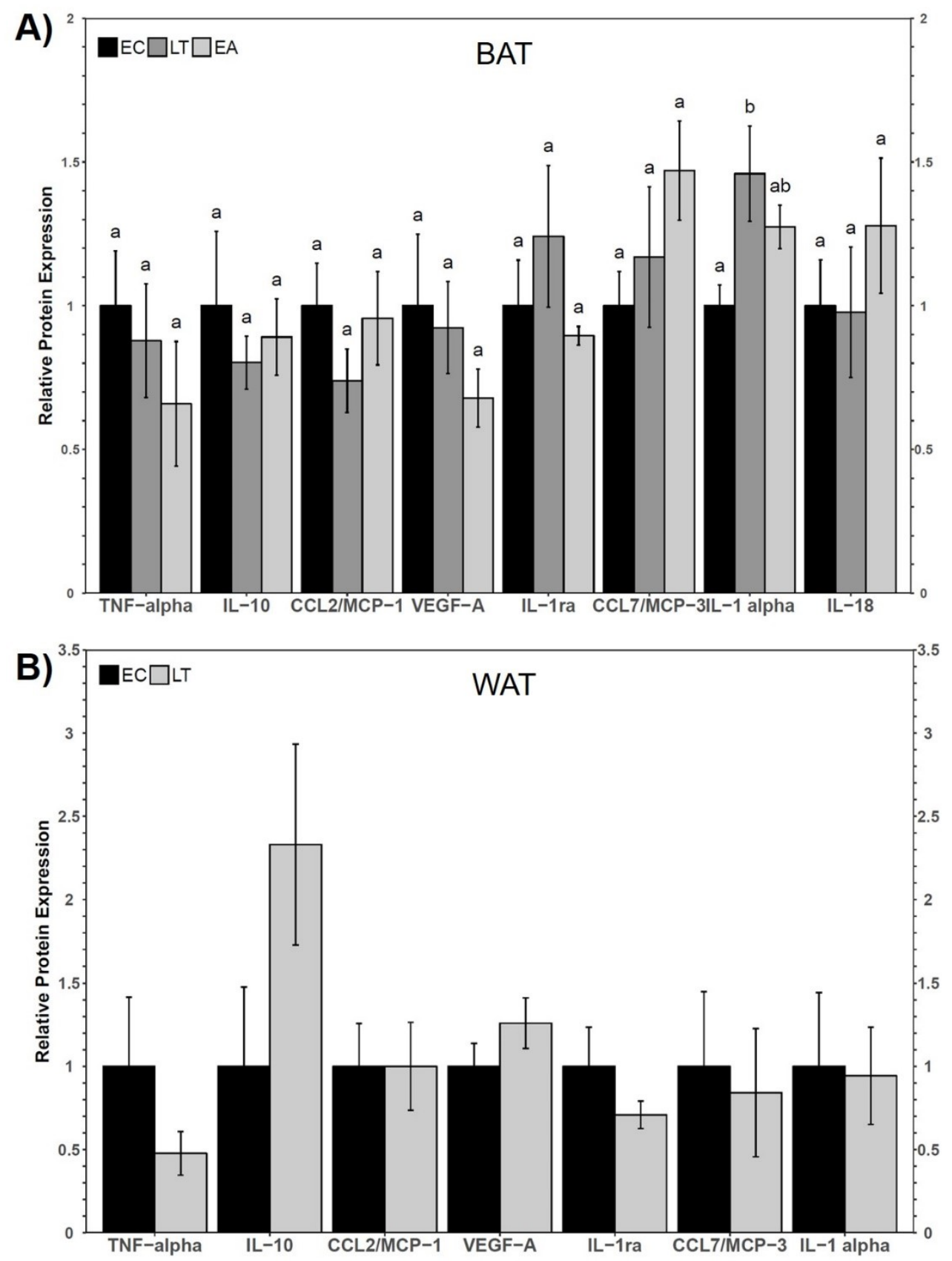

Figure 3.3: Relative fluorescence intensities of cytokine and growth factor analytes in A) BAT and B) WAT from 13-lined ground squirrels. Histogram showing mean standardized expression levels of cytokines and growth factors $( \pm$ SEM, $n=5$ independent BAT protein isolations or $n=4$ WAT protein isolations, except BAT VEGF-A was quantified with and $\mathrm{n}=4$ for EC, and BAT IL-18 was quantified with an $\mathrm{n}=4$ for LT. Exceptions for WAT include the use of $\mathrm{n}=3$ for EC (TNF $\alpha$, IL-10, VEGF-A, IL-1Ra, and IL-18) and LT (CCL2, and IL-18)). A) when comparing three time points using a one-way ANOVA and a Tukey post-hoc test, different letters are used to indicate statistical significance $(p<0.05)$, whereas B) statistical significance would be indicated with an asterisk following the Student's t-test. 

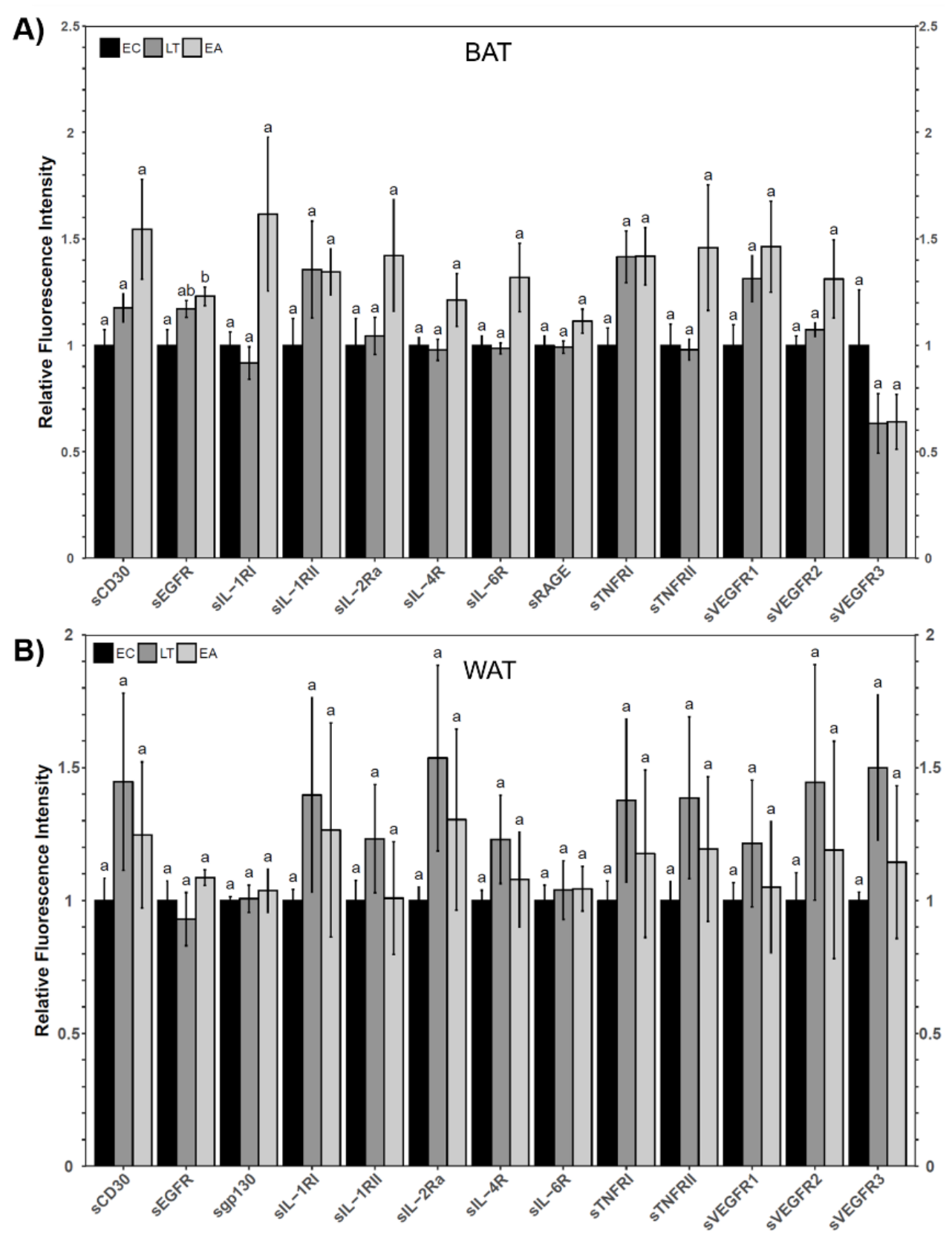

Figure 3.4: Relative fluorescence intensities of soluble receptors in A) BAT and B) WAT from 13-lined ground squirrels. Histogram showing mean standardized expression levels of soluble receptors $( \pm$ SEM, $n=$ 5 independent BAT protein isolations or $\mathrm{n}=4 \mathrm{WAT}$ protein isolations from different animals, except BAT sRAGE was quantified with EC $\mathrm{n}=3$ and $\mathrm{LT} \mathrm{n}=4$ and WAT sgp130 was quantified with EC and EA $\mathrm{n}=$ $3)$. Different letters are used to indicate statistical significance $(p<0.05)$, determined from a one-way ANOVA with a Tukey post-hoc test. 

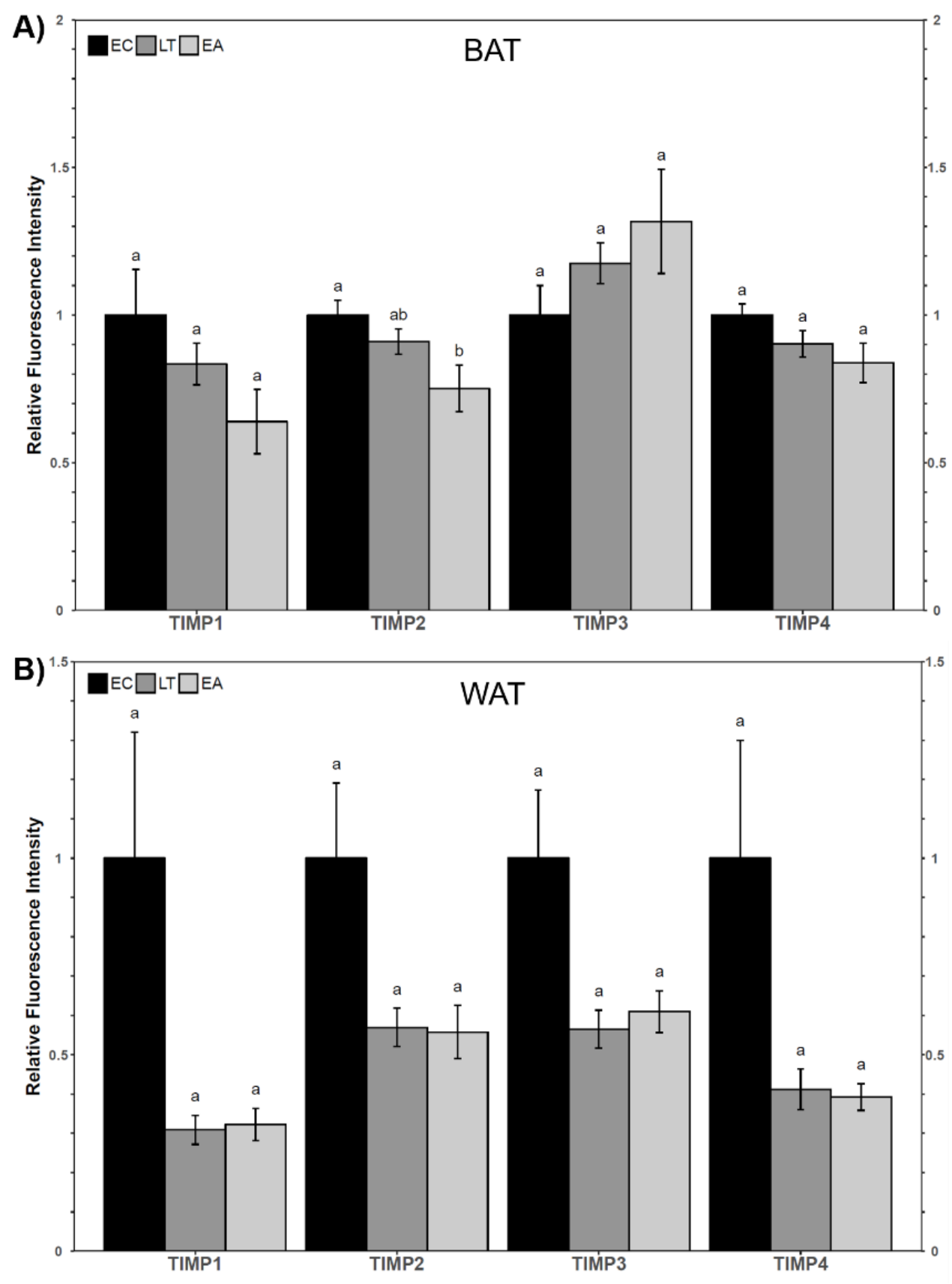

Figure 3.5: Relative fluorescence intensities of TIMP1-4 in A) BAT and B) WAT from 13-lined ground squirrels. Histogram showing mean standardized expression levels of TIMPs ( \pm SEM, $n=5$ independent BAT protein isolations or $n=4$ WAT protein isolations from different animals, except BAT LT and EA used $\mathrm{n}=4$ independent animals for protein preparations, and LT and EA used $\mathrm{n}=3$ for WAT. Different letters are used to indicate statistical significance $(p<0.05)$, determined from a one-way ANOVA with a Tukey post-hoc test. 


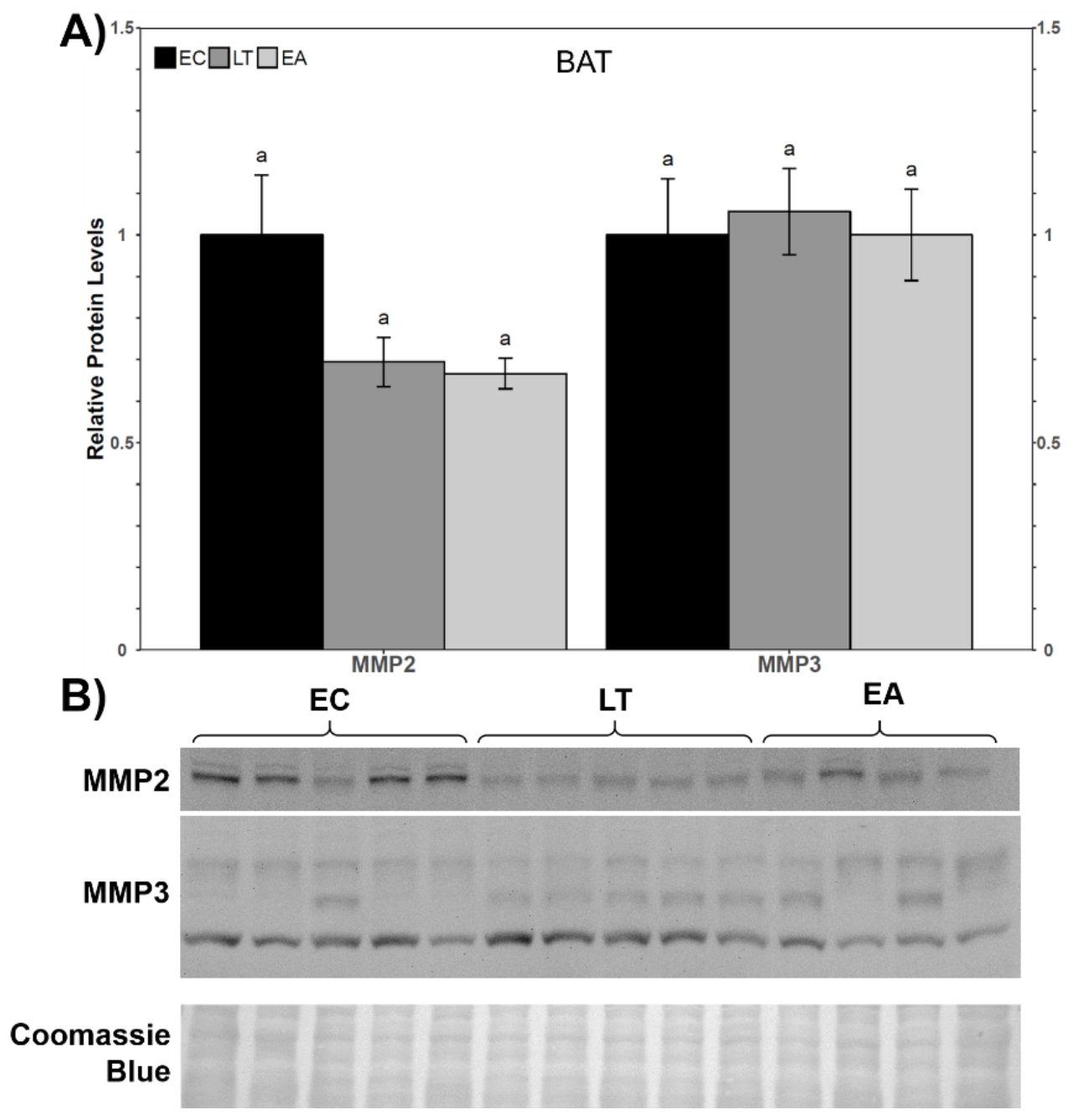

Figure 3.6: Relative protein levels of matrix metalloproteinases (MMPs) in ground squirrel BAT. A) Histogram showing mean standardized expression levels of MMP2 and MMP3 ( \pm SEM, $n=4-5$ protein isolations from independent animals). B) Quantified western blot bands and a set of representative bands from a Coomassie blue-stained membrane, used as the protein loading control. Data were analyzed using a one-way ANOVA with a Tukey post-hoc test. Shared letters indicate data that are not significantly different from each other and different letters indicate statistically significant differences between sample points $(\mathrm{p}<$ $0.05)$. 

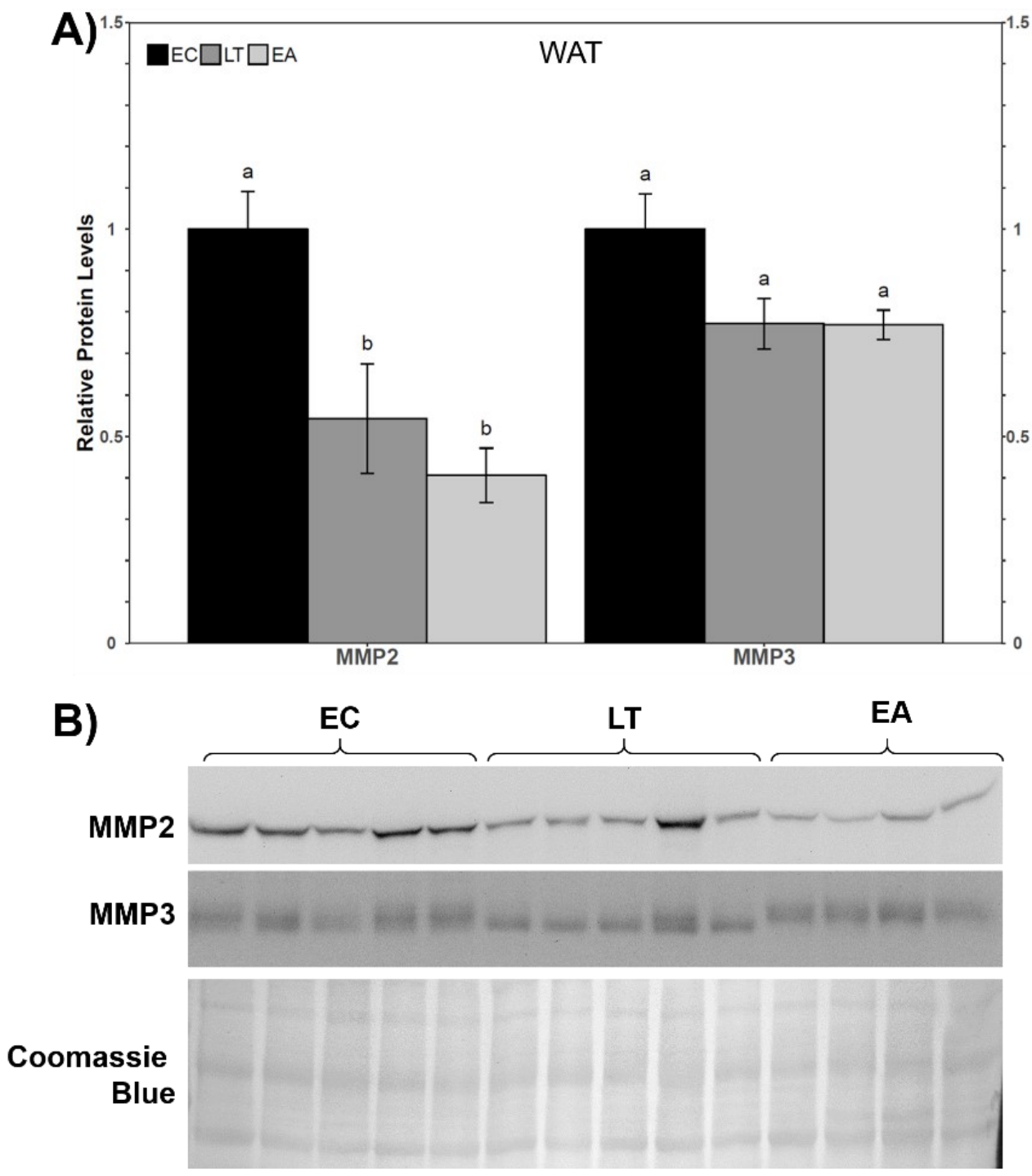

Figure 3.7: Relative protein levels of matrix metalloproteinases (MMPs) in ground squirrel WAT. A) Histogram showing mean standardized expression levels of MMP2 and MMP3 $( \pm$ SEM, $n=4-5$ protein isolations from independent animals). B) Quantified western blot bands and a set of representative bands from a Coomassie blue-stained membrane, used as the protein loading control. Data were analyzed using a one-way ANOVA with a Tukey post-hoc test. Shared letters indicate data that are not significantly different from each other and different letters indicate statistically significant differences between sample points $(\mathrm{p}<0.05)$. 


\section{MicroRNA expression patterns in the brown fat of hibernating 13-lined ground squirrels}




\subsection{Abstract}

The sequence diversity of microRNAs (miRNAs) allows these potent regulators of mRNA fate to bind multiple transcripts, giving them the power to inhibit diverse cellular processes. Therefore, miRNAs may regulate metabolic rate suppression (MRS, also termed torpor), an adaptation used by capable species to reduce energy expenditure, minimize tissue damage, and prolong life. The results of small RNA-sequencing of brown fat from euthermic in the cold room controls $\left(\mathrm{EC}, 37^{\circ} \mathrm{C}\right)$ and late torpid $\left(\mathrm{LT}, 5-8^{\circ} \mathrm{C}\right)$ ground squirrels revealed a central role for miRNAs in LT. Unsupervised clustering analysis of all 319 conserved miRNAs showed separation of EC and LT samples, which was supported by PCA analysis. Of the 76 miRNAs that were differentially expressed, 45 were upregulated during torpor. KEGG and GO analyses suggested these miRNAs inhibit genes within the ribosome, oxidative phosphorylation, and glycolysis/gluconeogenesis pathways. Some of the most downregulated miRNAs (miR-1-3p, miR-206 and miR-133a/b) had significant Pearson correlation coefficients, suggesting these myomiRs may be co-expressed in control animals. Only 3 of the 16 enriched KEGG pathways were less targeted by miRNAs during LT, including cytokine-cytokine receptor interactions and the coagulation and complement cascades, suggesting epigenetic or post-translation modifications may inhibit these potentially damaging processes. Alternatively, their activation could promote damage sensing, wound repair, and improve tissue homeostasis. Overall, miRNA-seq analysis of brown fat revealed a strong role for miRNAs in the downregulation of central metabolic processes necessary for MRS, and highlighted miRNAs that could be inhibited by antagomiRs to promote brown fat activity in potential obesity treatments, or that could be used to replicate torpor in non-hibernating mammals.

Keywords: Brown adipose tissue, Ictidomys tridecemlineatus, miRNA, metabolic rate suppression, gene expression, stress response 


\subsection{Introduction}

Metabolic rate suppression (MRS) allows capable animals to adapt to unpredictable and unfavourable environmental conditions via the inhibition of inessential energy-consuming processes. By decreasing metabolic rate by at least $95 \%$ and reducing core body temperatures to negative values in some species, hibernators can withstand months of harsh winter conditions using fatty acids from adipose depots as their primary source of cellular fuel (Storey, 2010). However, MRS is not simply a suppression of all cellular processes. It also involves increasing metabolic pathways that facilitate tissue homeostasis. For instance, hibernators differentially regulate epigenetic regulators that control DNA accessibility, to increase transcription of antioxidants enzymes, protein chaperones, anti-apoptotic proteins, and regulatory enzymes like kinases (Biggar et al., 2015; Rouble et al., 2013; Tessier et al., 2019). Developing our understanding of how animals facilitate MRS has enormous implications for advancing technologies that could prolong organ preservation for transplantation, improve treatments for human disease, and push the boundaries of space travel.

Brown adipose tissue (BAT) has medical importance given that it can dissipate energy as heat - a wonderful organ to exploit for weight loss strategies - but it can also regulate wholebody homeostasis through the release of adipokines including cytokines, growth factors, lipidderived hormones, and small non-coding RNA species (Lee et al., 2019; Maurizi et al., 2018). Adult humans have very small BAT depots with limited use in maintaining homeothermy but other mammals (Trayhurn, 2018), like hibernators, routinely use the thermogenic capabilities of BAT to rewarm the entire animal from $-2.9{ }^{\circ} \mathrm{C}$ (in the most extreme ground squirrels) to $37{ }^{\circ} \mathrm{C}$, in as few as 3 hours. Exploring the differences in gene expression between euthermic and torpid BAT would expose the mechanisms behind the magic that allow hibernators' BAT to coordinate heterothermy while maintaining tissue homeostasis. Learning from nature in this way could allow humans to exert more control over BAT activity, permitting increases in energy expenditure by turning on the genes that hibernators shut off, or permitting metabolic rate suppression in non- 
hibernating mammals, an innovation that would extend organ lifetime for transplant, and perhaps one day, apply to long-term space flight. For this reason, the current study sought to identify and characterize microRNAs expression patterns and their possible roles in the regulation of MRS in the BAT of a model hibernator, the 13-lined ground squirrels (Ictidomys tridecemlineatus).

Mature microRNAs (miRNAs) are a class of small (18 - $22 \mathrm{nt}$ ) and highly conserved noncoding RNA molecules that can inhibit virtually all biological functions via their complementary interactions to mRNA transcripts. Mature miRNAs differ from their precursors (hairpin miRNAs), in their sequence length (hairpin miRNAs are 60-70 nt long) and their secondary structure (hairpin miRNAs fold back on themselves, causing them to look like hairpins). One miRNA can target multiple transcript sequences and a single mRNA can be bound by a variety of miRNAs. Generally, imperfect binding results in the sequestration of the targeted mRNA to ribonucleoprotein granules in the cytoplasm for storage, whereas perfect complementarity to the target mRNA results in its degradation by endonucleases (Iadevaia and Gerber, 2015; Wang et al., 2017). Knowing that the complementarity of the seed region (approximately the first 8 nucleotides) to the target mRNA is an important factor to consider when assessing the functionality of binding miRNA targets (Jo et al., 2015), it was possible to use RT-qPCR experiments to examine the expression of selected miRNAs with known sequences. Using primers specific for the first $15-17$ bp of miRNA, ground squirrel BAT was shown to downregulate 6 of 20 investigated miRNAs (Wu et al., 2014). Downregulation refers to a decrease in biomolecule levels relative to the control condition, which could be due to the active suppression of the expression of the biomolecule or an increase in its degradation or efflux from the tissue. Mitochondrial functions such as beta-oxidation and uncoupling of the proton motive force from ATP generation, and the MAPK signaling pathway were suggested to be less inhibited during torpor. The current study was designed to investigate the expression of all conserved miRNAs in ground squirrel BAT. This workflow mapped each miRNA to its validated and 
predicted mRNA targets, and assessed miRNA influence over molecular pathways using KEGG Pathways and GO terms. Furthermore, random forest feature selection was performed to identify a subset of miRNAs that aid in the characterization of euthermic in the cold room control (EC) or late torpor (LT) samples, which may help characterize the evolutionary history of hibernation and related physiological adaptations. 


\subsection{Materials and Methods}

\subsubsection{Animals}

Male ground squirrels (Ictidomys tridecemlineatus) weighing 150-300 g were wild captured by a United States Department of Agriculture licensed trapper (TLS Research, Bloomingdale, IL) and transported to the laboratory of Dr. J.M. Hallenback at the Animal Hibernation Facility, National Institute of Neurological Disorders and Stroke (NINDS) (NIH, Bethesda, MD) where the hibernation experiments were conducted. Animal housing and experimental procedures were approved by the NINDS animal care and use committee (ACUC). Each 13-lined ground squirrel was fitted with a sensor chip (IPTT-300; Bio Medic Data Systems) injected subcutaneously while anesthetized with 5\% isoflurane and was housed individually in a shoebox cage at $21{ }^{\circ} \mathrm{C}$. Animals were fed a standard rodent diet and water ad libitum until they gained sufficient lipid stores to enter hibernation. Animals that weighed between 220 and $240 \mathrm{~g}$ were placed in the hibernacula and were randomly allocated to control or hibernation groups. To enable a natural transition into torpor, animals were transferred to an environmental chamber at 5 ${ }^{\circ} \mathrm{C}$ in constant darkness. Body temperature $\left(\mathrm{T}_{\mathrm{b}}\right)$, time, and respiration rate were observed to determine sampling points, as previously described (Frerichs et al., 1994). The euthermic in the cold room control ground squirrels (EC) were housed in a cold-room and had a $\mathrm{T}_{\mathrm{b}}$ of $37^{\circ} \mathrm{C}$ at the time of euthanasia. The stress condition (late torpor, LT) contained animals that were continuously in deep torpor for at least 5 days with $\mathrm{T}_{\mathrm{b}}$ values of $5-8{ }^{\circ} \mathrm{C}$. All 13-lined ground squirrels had been through torpor-arousal bouts prior to euthanasia by decapitation and tissue sampling, as previously described (McMullen and Hallenbeck, 2010). Tissue samples were shipped to Carleton University on dry ice and then stored at $-80{ }^{\circ} \mathrm{C}$ until use.

\subsubsection{RNA isolation and small RNA sequencing}

Approximately $70 \mathrm{mg}$ of frozen interscapular brown adipose tissue (BAT) was collected from four individual EC and LT ground squirrels. Eight frozen samples, each weighing $70 \mathrm{mg}$, were individually homogenized in $1 \mathrm{~mL}$ TRIzol reagent (Invitrogen; \#15596-018) using a 
Polytron homogenizer. Following the addition of $200 \mu \mathrm{L}$ of chloroform, the samples were inverted, and allowed to precipitate at room temperature before 15 min of centrifugation at 8,960 $\times \mathrm{g}$, at $4{ }^{\circ} \mathrm{C}$. The supernatant was collected and the TRIzol method was repeated to reduce contamination from lipid species. RNA within the supernatant was allowed to precipitate at room temperature for 10 min following the addition of $500 \mu \mathrm{L}$ of isopropanol. The RNA pellets were washed with $70 \%$ ethanol and centrifuged again for $5 \mathrm{~min}$ at room temperature. The ethanol was decanted and the RNA pellets were allowed to air dry before being resuspended in $50 \mu \mathrm{L}$ of RNase-free water. A BioTek Take3 multi-volume plate and spectrophotometer was used to determine $260 / 280 \mathrm{~nm}$ ratios and concentration of each sample. The integrity of each sample was further assessed through the use of $1 \%$ agarose gel electrophoresis, where SYBR-Green staining was used to confirm the presence of clear $28 \mathrm{~S}$ and $18 \mathrm{~S}$ rRNA bands.

Small RNA sequencing was performed by Canada's Michael Smith Genome Sciences Centre (British Columbia, Canada) using an Illumina NextSeq500 instrument generating singleend 75 base reads. Quality control of the final library was performed using Qubit and Agilent DNA 1000 Series II assays prior to sequencing.

\subsubsection{Data processing}

Raw sequences obtained from small RNA-sequencing were submitted to the SRA database and can be accessed with the following accession number (PRJNA662913). The raw reads were analyzed in a Unix shell environment in advance of differential miRNA abundance analysis in the R environment, as previously described (Zhang et al., 2016). Initial quality of the sequences was determined with a FastQ Quality Filter analysis. Then, Cutadapt version 0.10.1 was used to remove adapters, the 6 random nucleotides between the adapters and the small RNA sequences, and any reads shorter than 10 nucleotides in length, using a quality cutoff of 20 .

Using Bowtie with default settings, the trimmed sequences were aligned with a library of small non-coding RNAs (rRNA, tRNA, piRNAs, snoRNAs, and snRNAs) sequences sourced 
from the databases Rfam and piRNABank (Kalvari et al., 2018; Sai lakshmi and Agrawal, 2008). Sequences that aligned with the negative reference library were filtered out and any sequences that did not align to the negative reference library were aligned with sequences within a library of mature miRNA sequences from all organisms, sourced from miRBase version 22 (Kozomara et al., 2019). Aligned sequences, which had seed sequence lengths of 20 and perfect alignments to the sequences in the mature miRNA reference library were used for the analyses presented herein. SAMtools was used in the UNIX shell to index each read count with its miRNA identity into the SAM format such that it could be subsequently analyzed using the R platform (Li et al., 2009). MicroRNAs with read counts less than 10 were not included in the analysis. The read count data from individual fat samples ( $\mathrm{n}=4$ biological replicates from EC and LT conditions) were used. RBioArray was the R package that was used to normalize the data and to generate many of the figures. RBioArray uses a limma approach, where the RNA-seq read counts are normalized using $\log 2$-counts-per-million $(\log \mathrm{CPM})$ and the voom method is used to calculate read count variance for each normalized observation (Law et al., 2014).

\subsubsection{Differential gene expression, supervised and unsupervised cluster analysis}

Using the normalized and filtered miRNA expression data, the R package RBioArray was used call functions from other packages, including limma. First, an empirical Bayes test was performed to linearly model the miRNA expression data and determine which genes were differentially expressed during torpor, relative to the euthermic control group. Then, to determine which miRNAs were differentially expressed in similar or opposite manners, the Ward method was employed to cluster miRNAs based on their expression patterns in all samples (Ward, 1963). In addition to clustering similarly expressed miRNAs from all samples, the unsupervised cluster heatmap also grouped the samples via dendrograms based on similarity of miRNA expression patterns. A heatmap of unsupervised hierarchical clustering with dendrograms was also created. The unsupervised clustering used Euclidean distance between the vectors (i.e. $\operatorname{sqrt}\left(\operatorname{sum}\left(\mathrm{x}_{\mathrm{i}}-\mathrm{y}_{\mathrm{i}}\right)^{2}\right)$ and Ward clustering method (Ward, 1963). Differentially expressed miRNAs $(\mathrm{q}<0.01)$ were 
plotted as a volcano plot to show highly significant changes clearly but fold changes greater than $1.5(\mathrm{q}<0.05)$ were considered statistically significant.

Principal component analysis (PCA) was performed using the RBioFS package. A centered log-ratio transformation was applied to raw read counts (Quinn et al., 2018), and then a PCA biplot was produced showing the linearly correlated control and stress samples based on their gene expression profiles, with miRNAs superimposed on the plot to help identify the miRNAs responsible for the most variance between control and stress (torpor) samples.

Pearson correlation was used in the correlation cluster analysis to determine how strongly the 30 DE microRNAs $(q<0.01)$ were linearly correlated with one another, for EC and LT groups separately analyzed, where statistically significant correlations had $\mathrm{p}<0.05$. These results were graphed as correlation plots, where -1 represents highly negative correlations, 1 represents highly positive correlations, and 0 represents no correlation.

\subsubsection{Functional characterization of miRNA expression patterns}

RbiomirGS was used to map differentially expressed miRNAs to target mRNAs based on validated miRNA:mRNA interactions sourced from several online databases to ensure all interactions were considered for analysis (Zhang and Storey, 2018). Once potential miRNA:mRNA interactions were identified, an algorithm published by Zhang and Storey (2018) was used to create regulation scores (S score) for each mRNA target based on miRNA expression profiles. Logistic regression-based gene set enrichment used both the miRNA and mRNA enrichment scores, where all miRNAs in the gene list were used for the functional characterization of miRNA expression patterns. Enriched gene sets were analyzed against Gene Ontology (GO) terms and Kyoto Encyclopedia of Genes and Genomes (KEGG) gene sets to predict which pathways and cellular functions were significantly affected by changes in miRNA expression. A high coefficient indicates a pathway that is less downregulated by miRNA during $\mathrm{LT}$ relative to EC, and a negative coefficient indicates a pathway that may be negatively regulated 
by miRNAs during LT, relative to EC. Significantly enriched KEGG and GO pathways had $\mathrm{q}<$ 0.05. Finally, Pathview was used to visualize gene enrichment data and KEGG pathways of interest (Luo et al., 2017). Genes within KEGG pathways are coloured with mRNA S scores, with proposed downregulated genes shown in blue and potentially upregulated genes shown in red. S scores above 20 or below negative 20 are shown as the most saturated colour (red or blue) possible.

\subsubsection{Random forest feature selection and partial least squares discriminant analysis}

RBioFS was also used to identify a subset of miRNAs that could be used to characterize a sample's "condition", whether that be EC or LT. To identify a more concise subset of features (miRNAs), filtered raw reads for the 76 differentially expressed miRNAs $(\mathrm{q}<0.05)$ were used in random forest decision tree modelling and feature selection, as previously published (HadjMoussa et al., 2020b; Zhang et al., 2016). Filtered raw reads for the 76 miRNAs were center scaled and quantile normalized before recursive random forest (RF) variable importance (VI) was calculated for each feature (miRNAs). A bootstrapping random forest algorithm generated ntrees $=1001$, using ntimes $=50$ as the number of random forest VI computation runs. The output was an object with VI values for each feature and out of box (OOB) error rate. Then, a PCA plot of the 64 resulting miRNAs and their relationship to conditions and samples was generated using centered log transformed (CLR)-transformed reads to examine how well EC and LT groups were separated.

To further explore if the subset of recursive random forest feature selected miRNAs would be appropriate for characterizing control and torpor samples, PLS-DA was performed as previously described (J. Zhang et al., 2020). Briefly, a PLS model was constructed using the maximum number of components possible when using the SIMPLS PLS-DA modelling method and "leave one out" cross validation. Then, an optimal number of components was determined by finding the point at which the root mean squared error of prediction (RMSEP) of the model 
reached its first minimum within one standard error. The model was rebuilt using the optimal number of components $($ ncomps $=1)$ and a score plot was generated to show the supervised clustering of the EC and LT samples. A permutation test was done to determine if the final optimized model could function to classify samples vs. 999 similarly constructed models, where p $<0.05$ would indicate that the data could be confidently identified as being from an EC or LT group.

\subsubsection{RNA polyadenylation of miRNA and cDNA synthesis of miRNA}

To validate the expression patterns of a representative set of miRNAs, RNA was prepared as described above and the following steps were used to convert the RNA into miRNA cDNA. A Take3 and a BioTek spectrophotometer was used to assess RNA concentration and purity. Equal amounts $(2 \mu \mathrm{g})$ of RNA was diluted in $7.5 \mu \mathrm{L}$ of autoclaved $\mathrm{ddH}_{2} \mathrm{O}$ for each sample $(\mathrm{n}=4$ biological replicates of BAT sampled from EC and LT ground squirrels) before adding $1.0 \mu \mathrm{L}$ of 10X Poly-A polymerase buffer, $1.0 \mu \mathrm{L}$ of $1 \mathrm{mM}$ ATP and 0.5 Units of $E$. coli poly(A) polymerase from the Poly-A polymerase tailing kit (Lucigen, \#PAP5104H) to each reaction. Using a thermocycler, the reaction proceeded at $37^{\circ} \mathrm{C}$ for $30 \mathrm{~min}$ and then the adenylation was terminated by increasing the temperature to $95^{\circ} \mathrm{C}$ for $5 \mathrm{~min}$. The polyadenylated samples were put on ice for 5 minutes before adding $5 \mu \mathrm{L}$ of the Reverse Transcriptase miRNA Oligo-dT adapter primer (250 $\mathrm{pM}$ ) to each sample and incubating them at $95^{\circ} \mathrm{C}$ for $5 \mathrm{~min}$ and $60^{\circ} \mathrm{C}$ for $5 \mathrm{~min}$. Samples were placed on ice for at least $5 \mathrm{~min}$. To the template, $10 \mu \mathrm{L}$ of a reverse transcriptase master mix containing $5 \mu \mathrm{L}$ First-strand synthesis buffer, $2 \mu \mathrm{L}$ DTT, $1 \mu \mathrm{L} 25 \mathrm{mM}$ dNTPs, $1 \mu \mathrm{L}$ of the reverse transcriptase enzyme, and $1 \mu \mathrm{L}$ RNase-free water was added. The reverse transcription protocol was as follows: $16^{\circ} \mathrm{C}$ for $30 \mathrm{~min}$, and then 45 cycles of $20^{\circ} \mathrm{C}$ for $30 \mathrm{sec}, 42^{\circ} \mathrm{C}$ for $30 \mathrm{sec}$, and 50 ${ }^{\circ} \mathrm{C}$ for $1 \mathrm{sec}$, and completing the reaction at $85^{\circ} \mathrm{C}$ for $5 \mathrm{~min}$. The resulting miRNA cDNA was then diluted 10 -fold in autoclaved $\mathrm{ddH} 2 \mathrm{O}$ before preparing a pooled sample to assess primer efficiency and specificity. 


\subsubsection{RT-qPCR amplification of miRNA}

A standard curve using cDNA samples of increasing dilution was made for each primer set to verify that the primers were specific for the miRNA or mRNA transcript of interest and that primer-dimers were not being amplified. Only primer sets were used for quantification that generated a standard curve with a slope approaching 3.3, and a single product was generated as defined by a single peak in the melt curve using increasing temperatures between $57^{\circ} \mathrm{C}$ and 95 ${ }^{\circ} \mathrm{C}$. Supplemental Table 4.8 contains the primers used in these experiments. RT-qPCR using a BioRad CFX Connect Real-Time System began with an initial 3 min step denaturation at $95^{\circ} \mathrm{C}$. MiRNA amplification was done using 40 cycles of $95^{\circ} \mathrm{C}(10 \mathrm{sec})$ and $57^{\circ} \mathrm{C}(30 \mathrm{sec})$.

\subsubsection{Quantification and statistical analysis of RT-qPCR data}

The mean $\mathrm{Cq}$ value from technical replicates was used to calculate the relative quantity

$\left(2^{\wedge-\mathrm{Cq}}\right)$ for every biological replicate $(\mathrm{n}=4 \mathrm{EC}$ and $\mathrm{LT}$, each). Then, each value was normalized against the expression of the reference gene, snord-68, giving the $\Delta \Delta \mathrm{Cq}$ value. Normfinder analysis of a set of eight genes that were not differentially abundant between EC and LT conditions (Student's t-test, $\mathrm{p}>0.05$ ) identified snord-68 as the best reference gene based on its low inter- and intra-group variation, as well as its high stability. Outliers were identified using the Dixon test with a confidence level of $90 \%$. Outliers were removed from the dataset such that $n=3$ control biological replicates were used for miR-30e-5p, miR-101-3p, miR-376c-3p, and miR1260b. The mean $\Delta \Delta \mathrm{Cq}$ value and standard error of the mean was calculated for EC and LT before plotting torpor miRNA levels relative to the control, which was set to 1 for graphing purposes. Statistical analyses and graphing was performed using RBioplot (Zhang and Storey, 2016) and changes were considered statistically significant the Student's t-tests yielded a result of $\mathrm{p}<0.05$ (Zhang and Storey, 2016). 


\subsection{Results}

\subsubsection{Read counts of conserved miRNAs following small RNA-sequencing analysis}

Following the pre-processing steps where low-quality reads, adapters, short sequences $(<$

$17 \mathrm{bp}$ ), and sequences corresponding to non-miRNA small RNA species were removed, there was a total of 85,438,181 reads from four EC samples and 86,305,839 reads from three LT samples. Focusing only on the mature and conserved miRNAs, i.e. the miRNAs that mapped perfectly to known human miRNA sequences, the EC BAT samples had an aggregated read count of 3,821,664 and the LT BAT samples had 3,305,790 reads.

Reads counts for miR-1-3p and miR-206 were more closely inspected due to high variability in the expression of these miRNAs, based on the results of the RT-qPCR experiments. For the control samples 1-4, the raw read counts for miR-1-3p were also variable: 8790,2377 , 22778, and 112016, and the raw read counts in torpor BAT samples 5-8 were more similar at 179, 97, 211, and 257. Similarly, miR-206 raw read counts were $697,492,8775$, and 37745 for samples 1-4 and samples 5-8 had raw read counts 97, 35, 29, and 57. By contrast, the read counts for differentially regulated miRNA such as miR-1260b and miR-30e-5p were stable within treatment groups.

\subsubsection{Differential gene expression analysis of conserved miRNAs}

Small RNA sequencing analysis detected 319 conserved miRNAs in the 13-lined ground squirrel BAT samples (Supplemental Table 4.1). Using $\mathrm{q}<0.05$ and only considering miRNAs that changed expression levels more than 1.5 -fold the control value, there were 76 miRNAs that were differentially expressed during LT relative to EC. Of these, 45 were upregulated during LT and 31 were downregulated. Figure 4.1 shows the 30 miRNAs that were differentially expressed $(q<0.01)$. Some of the most upregulated miRNAs during LT included hsa-miR-365b-5p, hsamiR-541-5p and hsa-miR-186-5p, whereas hsa-miR-206, hsa-miR-1-3p and hsa-miR-5100 were highly downregulated during LT $(\mathrm{q}<0.01)$. 


\subsubsection{Cluster and principal component analyses}

In supervised cluster analysis, samples from control ground squirrels are purposefully grouped together to identify miRNAs clusters with similar expression patterns during LT (Figure 4.1B). For instance, miR-6529-5p miR-99b-5p miR-92a-3p, and miR-652-3p were similarly expressed. Of the EC samples, samples 1 and 3 expressed miRNAs in a similar fashion, whereas samples 5 and 7 had miRNA expression patterns similar to each other than to samples 6 or 8 . The unsupervised hierarchical cluster analysis of centered log transformed (CLR) global miRNA read counts indicated that the samples do indeed segregate into perfect EC and LT groups (Figure 4.2A). Dendrogram analysis further revealed tight groupings of miRNAs based on their expression patterns. Interestingly, some of the miRNAs had nearly identical expression patterns in each of the samples, such as miR-133b, miR-133a-5p and miR-133a-3p. These miRNAs appeared along with miR-206 and miR-3p in the same quadrant of the Principal component analysis (PCA) biplot (Figure 4.2B). PCA is a tool to visually represent the variance in a data as linear vectors on a coordination plot. It was used to help reveal both the shape of the expression data as well as the presence of key features that may explain the variance in the dataset. The PCA biplot (or relative variance biplot) showed distinct groupings of EC samples (pink circles) and LT samples (blue triangles), represented by distinct ellipses, as well as miRNA features that contribute to the variance observed between EC and LT samples. PCA revealed that PC1 explained $57.86 \%$ of the variance in the dataset, and PC2 explained $12.19 \%$ of the variance. Together, components 1 and 2 explain $70.05 \%$ of the variance in the dataset. The PCA biplot (or relative variance biplot) showed distinct groupings of EC samples (pink circles) and LT samples (blue triangles), represented by distinct ellipses, as well as miRNA features that contribute to the variance observed between EC and LT samples.

Pearson correlation heatmaps and significance plots were generated for the 30 differentially expressed miRNAs $(q<0.01)$ (Figure 4.3, Figure 4.4, Supplemental Table 4.2) but statistics for all 76 differentially expressed miRNAs $(q<0.05)$ can be found in Supplemental 
Table 4.3. There were 33 pairs of miRNAs with correlated expression patterns in the EC samples, where 13 were negative Pearson correlations and 20 were positive Pearson correlations. Notably, four miRNAs significantly and positively correlated with each other in EC samples: miR-1-3p, miR-133a-3p, miR-206, and miR-361-3p. These data were summarized in Table 4.1. By contrast, there were 46 pairs of miRNAs that significantly correlated in LT BAT samples (Figure 5.4B).

\subsubsection{Gene set analysis}

Differential miRNA expression was analyzed using the R package RbiomirGS and several online databases to determine which miRNA:mRNA interactions could be occurring during LT, and how this may affect signaling pathways, biological processes and molecular functions (Zhang and Storey, 2018). Supplemental Table 4.4 reports all predicted miRNA:mRNA interactions. KEGG pathways, GO biological processes (GO BPs), and GO molecular functions (GO MFs) were identified and plotted as volcano plots with $\mathrm{q}<0.05$ (Figure 4.5). All gene set analyses for KEGG, GO BPs and GO MFs are available in Supplemental

\section{Table 4.5.}

Of the 16 significantly enriched KEGG pathways $(\mathrm{q}<0.05)$, downregulated pathways such as ribosome, cell cycle, glycolysis and gluconeogenesis, and oxidative phosphorylation are discussed in the context of miRNA regulation of metabolic rate suppression (Figure 4.5A, Supplemental Figure 4.1). Similarly, 172 of 257 GO biological processes were downregulated (Figure 4.5B, Supplemental Table 4.5). GO BPs more inhibited during LT could be categorized into gene expression, RNA processing, and protein processing. KEGG pathways that were deemed less inhibited by miRNAs during LT including the complement and coagulation cascade and cytokine-cytokine receptor interactions are also discussed. Interestingly, although cytokinecytokine receptor interactions were generally less inhibited by miRNAs during torpor, some classes of cytokines were more inhibited than other according to Pathview analysis

(Supplemental Table 4.5). Pathview analysis was used to visualize how miRNAs may target 
pathways of interest, using the mRNA S scores (Luo et al., 2017). For example, transforming growth factor beta (TGF $\beta$ ) family cytokines and class I helical cytokines may be more inhibited by miRNAs, but CXC subfamily of chemokines, IL-6/12-like cytokines, and IL-1-like cytokines are less inhibited by miRNAs. The 85 GO BPs that were positively enriched included those involved in immune/inflammatory processes and tissue repair and/or remodeling. Finally, there were 102 GO MFs that were significantly enriched $(q<0.05)$, where 89 were downregulated and 13 were upregulated (Supplemental Table 4.5).

\subsubsection{Random forest feature selection and partial least squares discriminant analysis} Of the 76 differentially expressed miRNAs $(q<0.05)$ that were used as the starting dataset for random forest generation and feature selection, 64 miRNAs with the highest VI scores and were selected as a subset of miRNAs that could be used to classify EC and LT samples (Supplemental Figure 4.3A, Supplemental Table 4.6). The PCA plot shows that this miRNA subset can differentiate EC and LT samples, with most (79.85\%) of the variance in the dimension of the first principal component (Supplemental Figure 4.3B). PLS-DA was then used to create a regression model using the selected miRNAs and test its efficacy in predicting the correct grouping, EC or LT. The optimized model was made with 1 component, which explained $79.81 \%$ of the covariance between the dependent and independent samples. The PLS-DA score plot shows complete separation between the EC and LT samples, samples 1-4 and 5-8, respectively (Supplemental Figure 4.4B). The permutation test revealed that the model could significantly classify EC $(p=0.011)$ and LT $(p=0.015)$ samples (Supplemental Figure 4.4A and C). The supplemental files include complete permutation RMSEP values for all 999 iterations, for both EC and LT comparisons (Supplemental Table 4.7).

\subsubsection{Validation experiments}

The expression patterns of six miRNAs identified as being differentially expressed in miRNA-seq experiments (miR-1-3p, miR-206, miR-30e-5p, miR-101-3p, miR-376c-3p, miR1260b) were validated using RT-qPCR (Figure 4.6). The levels of miR-1-3p and miR-206 were 
not statistically significant between EC and LT, though they trended towards significant, where miR-1-3p levels decreased by $83 \%$ compared to the control value $(p=0.164)$ and miR-206 levels decreased by $88 \%$ compared to EC $(\mathrm{p}=0.158)$. This was due to high biological variation of the EC samples (SEM 0.45). By contrast, miR-30e-5p $(p=0.028)$, miR-101-3p $(p=0.023)$, and miR-376c-3p $(p=0.003)$ decreased significantly, where levels fell to $47 \%, 53 \%$ and $50 \%$ of the EC value, respectively. Further, miR-1260b increased significantly $(\mathrm{p}=0.009)$ by 1.39 -fold of the EC level. 


\subsection{Discussion}

Brown adipose tissue facilitates the periodic rewarming of ground squirrels via nonshivering thermogenesis and may have roles controlling whole-body homeostasis as a producer and exporter of cytokines, growth factors, and potentially, miRNAs (Cannon and Nedergaard, 2004; Lee et al., 2019; Townsend and Tseng, 2012). Thus, miRNA-seq was used to identify miRNAs that may be differentially regulated during LT, and in doing so, pathways that may be regulated by these non-coding, inhibitory RNAs during torpor in the BAT from a naturally hibernating mammal.

\subsubsection{Differential expression of miRNAs during torpor and unsupervised clustering analysis}

Of the 319 miRNAs that were identified as having the same nucleotide sequence as humans, 76 were differentially expressed, fold change greater than 1.5 times, $q<0.05)$. Of these 76 miRNAs, 30 miRNAs were highly significantly differentially expressed, $\mathrm{q}<0.01$ (Figure 4.1). In both cases, there were more upregulated miRNAs than downregulated miRNAs during LT, suggesting ground squirrels actively upregulate miRNAs to negatively regulate cell processes during LT. All RT-qPCR-amplified miRNAs were significantly differentially expressed in the same direction as the miRNAs detected using sequencing, despite the use of different RNA preparations, suggesting the changes observed using miRNA-seq are reflective of miRNA expression patterns in BAT across hibernating ground squirrels (Figure 4.6). Notably, RT-qPCRamplified miR-1-3p and miR-206 did not differ significantly between EC and LT samples, which will be explained in more detail later in the discussion. Further, there were differences between the miRNA expression patterns deduced in this study and those discovered in a smaller, more targeted study on 13-lined ground squirrel BAT (Wu et al., 2014). For example, miR-138 was found to increase in BAT during LT (Wu et al., 2014), but it was not identified as one of the 319 conserved miRNAs. Thus, the ground squirrel miR-138 sequence must differ from the human sequence by at least one nucleotide or read counts of miR-138 were too low to be considered. The 
miRNAs that were downregulated in the targeted study were observed as miRNAs of the 319 conserved miRNAs, but they did not change expression during torpor. Small RNA sequencing does not use a single reference gene as a baseline, thereby reducing bias in the dataset. Instead, read counts are logarithmically transformed and then normalized for sequence depth so that read counts are expressed as $\log \mathrm{CPM}$ (counts per million).

The unsupervised cluster analysis of all 319 mapped miRNAs showed a perfect grouping pattern: LT samples were more closely related to each other, and EC samples were most similar to each other (Figure 4.2A). These results were supported by the PCA, which showed nonoverlapping ellipses for EC and LT samples (Figure 4.2B). Together, these data indicate that miRNAs are important in facilitating the adaption to winter conditions. Recursive random forest feature selection was used to identify a subset of miRNAs may be useful to distinguish LT from control BAT samples. Using the raw filtered read counts of all differentially regulated miRNAs $(q<0.05)$, random forest feature selection identified a subset of 64 miRNAs that could be used to classify BAT samples into EC or LT categories (Supplemental Figure 4.3A, Supplemental Table 4.6). PCA (Supplemental Figure 4.3B) and PLS-DA (Supplemental Figure 4.4B) revealed complete separation of EC and LT samples, where statistical analysis of the PLS-DA model identified $\mathrm{p}<0.05$, indicating a strong PLS-DA model was built using these 64 miRNAs. The identified miRNAs may be useful for future studies focusing on the phylogenetic distribution of cold-adapted species or in therapeutics aimed at replicating MRS in non-hibernating species. Future research should investigate these miRNAs as possible regulators of MRS in nonhibernating species. These miRNAs or a subset thereof may be useful to reduce the thermogenic set point in heterothermic mammals and reduce energy expenditure. For example, treatments replicating MRS could have protective effects during surgical procedures that limit oxygen and nutrient delivery to the heart or brain, and in organs awaiting transplant. 


\subsubsection{Most miRNAs were significantly upregulated}

Overexpressed miRNAs regulate energy metabolism, thermogenesis, and cell

cycle/development. Several differentially expressed miRNAs are discussed to explore their possible roles in facilitating metabolic suppression in torpid ground squirrel BAT.

\subsubsection{1 miR-365b-5p}

The second-most upregulated miRNA was miR-365b-5p. MiR-365b-5p and miR-193b are highly expressed as a miRNA cluster in BAT (Feuermann et al., 2013). Herein, miR-193b-5p was not differentially expressed in BAT during LT, suggesting miR-365b-5p may have miR193b-independent roles in BAT during LT. Transcript-miRNA interaction analysis revealed that mir-365b-5p can target a wide range of genes including High-mobility group AT-hook 2 (HMGA2), which is a gene known to change the structure of DNA and has been implicated in the regulation of adipogenesis, mesenchymal differentiation, and tumor development (Markowski et al., 2013). MiR-365b-5p targets some of the mRNAs found to have the most negative mRNA S scores, indicating strong downregulation of these genes when considering the expression patterns of all 319 mapped miRNAs. MiR-365b-5p can target actin gamma (ACTG1) and actin beta (ACTB), genes that, if inhibited, would limit cell growth and proliferation during torpor. Finally, miRNA-365-5p targets Ankyrin Repeat Domain 52 (ANKRD52), which functions in the dephosphorylation of Argonaute 2 (AGO2) following Casein Kinase 1 Alpha 1 (CSNK1A1) phosphorylation, so its possible inhibition by miR-365b-5p and other known inhibitors (e.g. the miR-17-92a cluster of miRNAs) could contribute to control over miRNA biogenesis during torpor (Golden et al., 2017; Jung et al., 2018). This would be consistent with a downregulation of GO MFs miRNA binding and 3'UTR mRNA binding (Supplemental Table 4.5).

\subsubsection{2 miR-92a-3p and miR-99b-5p}

Some miRNAs may be released from brown fat via 40-100 $\mathrm{nm}$ lipid vesicles called exosomes to invoke changes in distal organs (Chen et al., 2016; Lee et al., 2019). Two such miRNAs, miR-92a-3p and miR-99b-5p, were upregulated during LT. Mir-92a is downregulated 
in exosomes from cAMP-activated BAT (Chen et al., 2016). The inhibition of miR-92a promoted the expression of genes involved in BAT activity, including UCP1, Cytochrome C Oxidase Subunit 7A1 (COX7A1), and Peroxisome proliferator-activated receptor gamma coactivator 1alpha (PPARGC1A) (Z. Zhang et al., 2020) and increased in vitro brown adipocyte differentiation from precursor cells, mitochondrial biogenesis, and cell oxygen consumption rate (Z. Zhang et al., 2020). Therefore, miR-92a upregulation in the context of torpor could be part of a biochemical adaptation that limits energetically-expensive thermogenesis and cell differentiation processes when the organism is in a state of metabolic suppression. KEGG analysis supported miRNA-mediated suppression of genes involved in oxidative phosphorylation (Supplemental Figure 4.1).

Similarly, miR-99b-5p may be upregulated during LT to prevent fibroblast growth factor 21 (FGF21) activities until thermogenesis and oxidative phosphorylation are required during the arousal period. FGF21 is a BAT-secreted factor that regulates whole body homeostasis, is upregulated following $\beta 3$-adrenergic signaling (required for non-shivering thermogenesis), and is involved in regulating glucose uptake through glucose transporter 1 (GLUT1), glucose oxidation, lipolysis, and reducing fatty acid synthesis (de Jong et al., 2015; Ni et al., 2015). Though hibernators overexpress FGF21 in the liver during LT, perhaps to enhance the mobilization of fatty acid reserves to other organs (Wu and Storey, 2016), FGF21 transcript levels were too low to detect in the BAT of torpid ground squirrels (Hampton et al., 2013), which may be a consequence of miRNA-mediated gene inhibition.

\subsubsection{3 miR-1260b}

MiR-1260b was significantly overexpressed relative to the EC group, which was confirmed using RT-qPCR (Figure 4.6). This miRNA had similar expression patterns as miR30b-3p, miR-501-3p, miR-186-5p and miR-502-3p, based on supervised clustering (Figure 4.2B). However, Pearson correlation cluster analysis did not reveal any statistically significant 
associations among these miRNAs in EC or LT samples, which suggested that these miRNAs are not co-expressed (Figure 4.3, Figure 4.4). Recursive random forest feature selection identified miR-1260b as a possible feature to use in the classification of EC and LT BAT samples (Supplemental Figure 4.3). Similarly, miR-1260b was upregulated in the muscle of daily torpid lemurs and was identified as a marker of LT using recursive random forest feature selection (Hadj-Moussa et al., 2020b). Its function during torpor is not fully elucidated but it may target activating transcription factor ATF-6 beta (ATF6b, also known as CREBL1) (Supplemental

Table 4.4). ATF6b coordinates the unfolded protein response (UPR) and it is differentially expressed in several ground squirrel tissues (Mamady and Storey, 2008). However, ground squirrels upregulate other transcription factors (ATF4 and CAMP responsive element binding protein 1 (CREB1)) involved in the UPR (Mamady and Storey, 2008), so miRNA-mediated inhibition of ATF6b could reduce redundancy and conserve energy. Bats also upregulate ATF4 in liver (Huang et al., 2020), so further analysis of relative miRNA expression in hibernating bat liver and BAT is required to determine if miRNAs such as miR-1260 among others may also regulate the unfolded protein response in other mammalian hibernators.

\subsubsection{Ribosomes and translation}

Based on the expression of the 319 mapped miRNAs, there were more KEGG Pathways that were significantly downregulated than positively enriched (Figure 4.5A). The KEGG pathway that was found to be the most significantly enriched and more inhibited during LT was the ribosome, which correlated well with significantly downregulated GO BPs associated with RNA processing and translation (e.g. mRNA metabolic process, RNA processing and translational initiation) (Supplemental Table 4.5). It is well understood that hibernators prevent translation during torpor, as it is an energetically costly process. Translation uses almost a quarter of the available cellular oxygen in rat tissues at standard metabolic rate (Rolfe and Brown, 1997). For this reason, it makes sense that miRNAs in ground squirrel BAT and Myotis lucifugus WAT would target genes involved in translation (Yuan et al., 2015). Briefly, previous experiments on 
hibernators revealed a reduction in the incorporation of radiolabeled methionine into hepatic proteins relative to normothermic animals (Whitten and Klain, 1968). Ribosomes normally form polysomes along a mRNA to efficiently produce protein to satisfy the cell's requirements, but during torpor, there are statistically more monosomes than polysomes compared to arousing Arctic ground squirrels (Spermophilus parryii) (Knight et al., 2000). Over the years the mechanisms describing how hibernators suppress translation have come to light, where differential gene expression and protein phosphorylation are postulated to play major roles (Frerichs et al., 1998; Logan et al., 2019; Miyake et al., 2015; van Breukelen and Martin, 2001). For example, over 60 ribosomal protein genes are significantly more expressed in BAT from April euthermic ground squirrels compared to torpor or interbout arousal (Hampton et al., 2013). Therefore, statistical enrichment of the ribosome KEGG pathway and GO BPs is consistent with the literature that has shown marked downregulation of translation during torpor. Furthermore, the current study expands on the mechanism known to facilitate this energy-saving adaptation.

\subsubsection{Glycolysis and gluconeogenesis}

One of the cruxes of seasonal torpor in fat-storing mammals is a switch from glucose to lipid oxidation (Wang and Wolowyk, 1988), so it was no surprise that the glycolysis/gluconeogenesis KEGG pathway was enriched during LT in ground squirrel BAT. Nearly all major glycolysis enzymes from hexokinase to pyruvate kinase were identified as targets of miRNA regulation (Supplemental Figure 4.1), which was consistent with a significant downregulation of the GO BP called glycolytic catabolic process $(q=0.0001$, Supplemental Table 4.5). Glycolytic inhibition has been observed in other hibernators including the meadow jumping mouse (Zapus hudsonius), where glycogen phosphorylase activity was depressed during torpor in the liver due to decreased total and phosphorylated levels of the enzyme, and similar mechanisms were discovered to be used to suppress the activity of glycolytic enzymes 6phosphofructo-1-kinase and pyruvate kinase (Storey, 1987). These results suggest a role for miRNAs in the inhibition of glycolytic enzyme translation during torpor. In addition, L-lactate 
dehydrogenase may be inhibited by miRNAs, indicating that anaerobic glycolysis is not used to supplement cellular ATP in a possible attempt to limit lactate build up. Consistent with this, unpublished data on muscle LDH activity and regulation during hibernation in Richardson's ground squirrels shows that LDH has a significantly higher $\mathrm{K}_{\mathrm{m}}$ (lower affinity for lactate) during torpor compared to euthermia, when assayed at physiologically relevant temperatures, and LDH activity is inhibited by phosphorylation (Ruberto, 2015). Similarly, hibernating little brown bats (Myotis lucifugus) show decreased LDH activity in multiple tissues including flight muscle and liver (Moon, 1978). Thus, anaerobic glycolysis is inhibited during torpor at several levels of regulation and in several mammalian hibernators. Overall, the finding that the glycolysis and gluconeogenesis KEGG pathway is significantly enriched during torpor reinforces the notion that hibernators use diverse mechanisms to suppress glycolysis and the use of stored glucose such that beta oxidation of lipids is prioritized.

\subsubsection{Electron transport chain}

MiRNAs may also play a role in inhibiting the transcription of enzymes involved in each of the electron transport chain complexes, except for complex II. More specifically, NADHquinone oxidoreductase subunit A of complex I, ubiquinol-cytochrome c reductase cytochrome b/c1 subunit of complex III, cytochrome c oxidase cbb3-type subunit I of complex IV, and H+exchanging ATPase subunits of ATPases are potential targets of upregulated miRNAs in ground squirrel BAT during torpor. However, some complex I, III, and IV subunit transcripts are not significantly different comparing BAT samples from torpid and interbout aroused ground squirrels. For instance, complex I NADH dehydrogenase [ubiquinone] 1 alpha subcomplex subunit 9 (NDUFA9) and NDUFA10 transcripts are upregulated during the Fall and are maintained at elevated levels during the hibernation months, relative to spring aroused ground squirrels (Hampton et al., 2013). These genes are predicted to be inhibited by miRNAs during torpor, but rather than be degraded, they may simply be sequestered into stress granules until their synthesis is required. Indeed, the complexes of the electron transport chain maintain a higher 
respiratory capacity during the heterothermic months, relative to a spring aroused control (Ballinger et al., 2016). The proton pumping complexes may be inhibited until arousal, when fatty acids are oxidized, reducing equivalents are made, inner mitochondrial protons are pumped through UCP1 to facilitate non-shivering thermogenesis.

\subsubsection{Downregulated miRNAs may regulate cell differentiation and inflammation}

\subsubsection{Muscle-specific miRNAs (myomiRs)}

Downregulated miRNAs provide insights into pathways and processes that are less inhibited during LT and the most downregulated miRNAs during torpor were miR-1-3p and miR206 (Figure 4.1A). Supervised cluster analysis revealed that miR-206 and miR-1-3p had highly similar expression patterns in ground squirrel BAT (Figure 4.1B). These two miRNAs were observed on the volcano plot and on the PCA plot in the far left, away from most other miRNAs (Figure 4.2A). Another "myomiR", miR-133a, was also highly downregulated during LT (q < 0.05 ) and clustered with miR-1-3p and miR-206 within the EC ellipse on the PCA plot, signifying these miRNAs may describe much of the variance in the EC samples. RT-qPCR validation experiments also showed high variation in the expression of miR-1-3p and miR-206 in EC samples even though different RNA preparations were made for miRNA-seq and RT-qPCR experiments (Figure 4.6). The levels of relative miR-1 and miR-206 expression were so variable in the RT-qPCR experiments that the downward trend during LT was not significant. Since miRNA-seq is a more sensitive and specific approach compared to RT-qPCR, and these miRNAs were found to be significantly downregulated $(\mathrm{p}<0.01)$, there is high confidence that these miRNAs are indeed differentially expressed during LT.

Along with miR-133a/b, miR-1 and miR-206 are part of the same myomiR family, for their original identification in muscle tissue, and their similar sequences. Despite being called "muscle-specific" miRNAs, these miRNAs have been found in other tissues including interscapular and cervical BAT depots (Walden et al., 2009). MyomiRs are capable of regulating 
myogenesis and skeletal muscle regeneration, BAT cell maturation and beige-ing in white adipocytes (the process by which white adipocytes take on characteristics of brown adipocytes), vasodilation/vasoconstriction and angiogenesis, among other processes (Liu et al., 2013; Margolis et al., 2017; Shan et al., 2010; Stahlhut et al., 2012; Trajkovski et al., 2012; Walden et al., 2009). Since their expression patterns are consistent across the same control samples (e.g. high expression of miR-1-3p and miR-206 in samples 3 and 4, but lower expression in samples 1 and 2), these miRNAs may be co-expressed in individual ground squirrels. Consistent with this notion, the supervised Pearson correlation heatmap shows that miR-1-3p, miR-206, and miR133a-3p were all positively correlated in EC samples (Figure 4.3B, Table 4.1, Supplemental Table 4.3).

The characterization of the role of myomiRs in brown adipose tissue is relatively limited, with only a few BAT-specific targets identified. For example, miR-133 is able to target the 3'UTR of PR domain containing 16 (PRDM16) in mammalian BAT (Liu et al., 2013; Trajkovski et al., 2012). PRDM16 controls the differentiation of precursor cells into mature brown and beige adipocytes. When cells are exposed to cold stress, myocyte enhancer factor 2 (MEF2) downregulates the expression of miR-133, allowing for more PRDM16 to be produced, thereby enhancing the maturation of BAT cells and increasing downstream thermogenesis (Trajkovski et al., 2012). In addition to PRDM16, miR-133 downregulation was correlated with increases in the expression of thermogenic genes UCP1, PPAR $\gamma 2$, PGC1 $\alpha$ and cell death activator (CIDEA). Thus, the downregulation of miR-133 and likely other myomiRs in metabolically suppressed BAT may disinhibit BAT maturation and subsequent thermogenesis. The 13-lined ground squirrel BAT transcriptome supports a role for myomiR downregulation in the facilitation of thermogenesis to some extent, since MEF2D was identified as one of the most highly enriched transcription factors in ground squirrel BAT compared to white adipose tissue, and UCP1 is highly expressed in BAT during torpor compared to October ground squirrels (Hampton et al., 
2013). However, PRDM16 was noted as being very lowly expressed in ground squirrel BAT at some time points (Hampton et al., 2013), suggesting miR-133 downregulation may not correlate with Prdm16 levels during LT. Compared to other mouse tissues, like heart and skeletal muscle, BAT expresses miR-133a or miR-133b at a much lower level, which was postulated to be the result of residual cell differentiation (Endo et al., 2013). Thus, even in adult mouse tissues, myomiRs such as miR-133 may not play a major role in the regulation of brown adipocyte proliferation and differentiation. Further experiments are required to characterize what genes may be important for myomiRs to target in adult BAT.

GO BPs that appear to be positively enriched (less inhibited by miRNAs during LT) included those involved in sensing and responding to cell stress whereas GO MF terms were associated with chemokine/cytokine/growth factor activity and their binding to receptors (Figure 4.5). There are two explanations to account for these pathways being less inhibited by miRNAs during LT in BAT. First, it is possible that genes encoding cytokines, growth factors, chemokines, and coagulation proteins are less expressed in the first place, perhaps through epigenetic regulation of histones or the DNA itself. Secondly, miRNAs may inhibit pathways requiring these proteins to a lesser degree because they help maintain BAT homeostasis while the ground squirrel is metabolically suppressed.

\subsubsection{Cytokine-cytokine receptor interaction}

Cytokine-cytokine receptor interactions were generally less inhibited by miRNAs during LT in ground squirrel BAT (Supplemental Figure 4.2). However, class I helical cytokines and the TGF $\beta$ family of cytokines appeared to be more inhibited during torpor, except for TGFB1/2 and their receptors TGFBR1/2 and activin A receptor like type 1 (ACVRL1). This result agrees with transcriptome results that showed TGFBR2 to be more expressed during the hibernation period compared to April and October euthermic animals (Hampton et al., 2013). TGF $\beta$ signaling regulates a variety of biological processes including mesenchymal stem cell differentiation, cell 
cycle, and cell death, wound healing, cell-matrix interactions, and the immune response (Schuster and Krieglstein, 2002). Many GO BPs corresponding to these processes were less inhibited by miRNAs during torpor (Figure 4.5), suggesting the LT miRNA profile may permit TGF $\beta$ mediated processes like wound healing or chemotaxis to promote tissue homeostasis. The BAT transcriptome also supported a disinhibition of class II helical cytokine IL10RB and IL-6/12-like cytokine leukemia inhibitory factor receptor (LIFR), which were significantly more abundant during hibernation compared to April or October (Hampton et al., 2013). Finally, the BAT transcriptome results supported miRNA-mediated inhibition of activin a receptor type $2 \mathrm{~A}$ (ACVR2A) was highlighted as a possible target of miRNAs during torpor, since ACVR2A transcript levels were low during torpor compared to April control ground squirrels (23). Since transcript levels in torpid ground squirrels align relatively well with the genes predicted to be more or less inhibited by miRNAs during torpor, cytokines are likely less inhibited by miRNAs during LT and may play key roles in regulating metabolic rate suppression in BAT.

$\mathrm{CREB}, \mathrm{NF} \kappa \mathrm{B}$ and signal transducer and activator of transcription (STATs) can increase the expression of CXC family of chemokines and IL-6/12-like family of cytokines (Kusuyama et al., 2016; Lou et al., 2017), and these transcription factors may be more active during torpor. Phosphorylated (active) CREB increased during LT in the BAT of hibernating 13-lined ground squirrel (Mamady and Storey, 2008; Rouble et al., 2014). NFkB protein expression is no different between control and torpid states, but some of its downstream genes are more abundant at the protein level during torpor (e.g. c-myc, NLRP3, RAGE), suggesting it is also active during LT (Hadj-Moussa et al., 2020a; Logan and Storey, 2018; Samantha M. Logan and Storey, 2021). The interleukins IL-1 $\beta$, IL-6, LIF, and IL-11 were all less inhibited during LT and all share a common receptor called Interleukin 6 Signal Transducer (IL6ST, also known as Glycoprotein 130 or CD130) (Supplemental Figure 4.2). After these ILs bind IL6ST and its coreceptors, IL6ST dimerizes and activates STAT signaling (Murakami et al., 2019). Intriguingly, ground squirrel 
BAT IL6ST transcript levels were decreased during torpor compared to interbout arousal levels (Hampton et al., 2013), even though the miRNAs that are able to target it were largely downregulated (e.g. miR-1-3p, miR-19b-3p, miR-376c-3p, miR-148a-3p, etc.). There is evidence that IL-1 $\beta$ levels do not change during torpor but they are upregulated at the gene and protein levels in I. tridecemlineatus BAT during early arousal (Samantha M. Logan and Storey, 2021). Together, these results suggest that miRNAs likely do not bind and degrade the transcripts involved in cytokine receptor interactions, for they may be required during early arousal.

\subsubsection{Coagulation and complement cascades}

Blood clotting time increases in several species of hibernating ground squirrels $(S$. frankinii, Urocitellus columbianus, and I. tridecemlineatus), suggesting that changes in blood clotting dynamics may be universal among hibernating ground squirrels (Pivorun and Sinnamon, 1981). Blood clotting proteins are almost exclusively expressed in the liver, so it would be interesting to investigate if hibernator BAT can secrete miRNAs to regulate the translation of proteins in the liver. For example, Pathview analysis suggested that thrombin, the enzyme that cleaves fibrinogen into fibrin, was predicted to be targeted by miRNAs. Upregulated miRNAs miR-423-3p and miR-1260 could mediate thrombin gene expression, so future studies investigating the miRNA composition of BAT exosomes should focus on these miRNAs. Preventing clotting as $\mathrm{T}_{\mathrm{b}}$ is reduced from $37^{\circ} \mathrm{C}$ to $5-8{ }^{\circ} \mathrm{C}$ is necessary to promote blood flow through highly vascularized BAT, reduce injury to the blood vessels, and prevent stoke.

\subsection{Conclusion}

To better understand how miRNAs may regulate biological processes in the brown fat from a model mammalian hibernator, miRNA-seq was employed to identify and characterize miRNA expression patterns. Of the 319 miRNAs that aligned perfectly with human miRNA sequences, 76 were differentially regulated $(\mathrm{q}<0.05)$, and of the 30 DE miRNAs $(\mathrm{q}<0.01), 22$ were significantly upregulated. RT-qPCR validated the expression of several differentially 
expressed miRNAs. Global miRNA expression patterns were sufficient to perfectly separate EC and LT samples, further suggesting a key role for miRNAs in the facilitation of metabolic suppression. KEGG pathways that were statistically more suppressed by miRNAs during LT included the ribosome, glycolysis/gluconeogenesis, and oxidative phosphorylation. Upregulated miRNAs were proposed to inhibit genes involved in processes including cell differentiation and cell cycle, energy metabolism (insulin sensitivity, glucose or fatty acid catabolism, reduction of and fatty acid synthesis) and the unfolded protein response. Interestingly, myomiRs made up a cluster of significantly downregulated miRNAs, which was postulated to prevent maturation of pre-adipocytes and regulate the expression of thermogenic genes during torpor. Finally, cytokinecytokine receptor interaction and coagulation and complement cascades KEGG pathways were significantly less inhibited during LT. Cytokine signaling may play important roles in stressinduced cell signaling during LT, but coagulation cascades are likely inhibited by miRNAs and post-translational modifications to proteins. Together, the results of study suggest a strong role for miRNAs in the regulation of metabolic suppression in the BAT of 13-lined ground squirrels. 


\subsection{References}

Ballinger, M.A., Hess, C., Napolitano, M.W., Bjork, J.A., Andrews, M.T., 2016. Seasonal changes in brown adipose tissue mitochondria in a mammalian hibernator: from gene expression to function. Am. J. Physiol. - Regul. Integr. Comp. Physiol. 311, R325-R336. https://doi.org/10.1152/ajpregu.00463.2015

Biggar, K.K., Wu, C.-W., Tessier, S.N., Zhang, J., Pifferi, F., Perret, M., Storey, K.B., 2015. Primate torpor: regulation of stress-activated protein kinases during daily torpor in the gray mouse lemur, Microcebus murinus. Genomics. Proteomics Bioinformatics 13, 81-90. https://doi.org/10.1016/j.gpb.2015.03.002

Cannon, B., Nedergaard, J., 2004. Brown adipose issue: function and physiological significance. Physiol. Rev. 84, 277-359. https://doi.org/10.1152/physrev.00015.2003

Chen, Y., Buyel, J.J., Hanssen, M.J.W., Siegel, F., Pan, R., Naumann, J., Schell, M., van der Lans, A., Schlein, C., Froehlich, H., Heeren, J., Virtanen, K.A., van Marken Lichtenbelt, W., Pfeifer, A., 2016. Exosomal microRNA miR-92a concentration in serum reflects human brown fat activity. Nat. Commun. 7, 11420. https://doi.org/10.1038/ncomms11420

de Jong, J.M.A., Larsson, O., Cannon, B., Nedergaard, J., 2015. A stringent validation of mouse adipose tissue identity markers. Am. J. Physiol. Metab. 308, E1085-E1105. https://doi.org/10.1152/ajpendo.00023.2015

Endo, K., Weng, H., Naito, Y., Sasaoka, T., Takahashi, A., Fukushima, Y., Iwai, N., 2013. Classification of various muscular tissues using miRNA profiling. Biomed. Res. 34, 289-299. https://doi.org/10.2220/biomedres.34.289

Feuermann, Y., Kang, K., Gavrilova, O., Haetscher, N., Jang, S.J., Yoo, K.H., Jiang, C., Gonzalez, F.J., Robinson, G.W., Hennighausen, L., 2013. MiR-193b and miR-365-1 are not required for the development and function of brown fat in the mouse. RNA Biol. 10, 1807-14. https://doi.org/10.4161/rna.27239

Frerichs, K.U., Kennedy, C., Sokoloff, L., Hallenbeck, J.M., 1994. Local cerebral blood flow during hibernation, a model of natural tolerance to "cerebral ischemia." J. Cereb. Blood Flow Metab. 14, 193-205. https://doi.org/10.1038/jcbfm.1994.26

Frerichs, K.U., Smith, C.B., Brenner, M., DeGracia, D.J., Krause, G.S., Marrone, L., Dever, T.E., Hallenbeck, J.M., 1998. Suppression of protein synthesis in brain during hibernation involves inhibition of protein initiation and elongation. Proc Natl Acad Sci USA 95, 14511-14516. https://doi.org/10.1073/pnas.95.24.14511

Golden, R.J., Chen, B., Li, T., Braun, J., Manjunath, H., Chen, X., Wu, J., Schmid, V., Chang, T.-C., Kopp, F., Ramirez-Martinez, A., Tagliabracci, V.S., Chen, Z.J., Xie, Y., Mendell, J.T., 2017. An Argonaute phosphorylation cycle promotes microRNA-mediated silencing. Nature 542, 197-202. https://doi.org/10.1038/nature21025

Hadj-Moussa, H., Wijenayake, S., Storey, K.B., 2020a. Multi-tissue profile of NFkB pathway regulation during mammalian hibernation. Comp. Biochem. Physiol. Part - B Biochem. Mol. Biol. 246-247. https://doi.org/10.1016/j.cbpb.2020.110460

Hadj-Moussa, H., Zhang, J., Pifferi, F., Perret, M., Storey, K.B., 2020b. Profiling torpor-responsive microRNAs in muscles of the hibernating primate Microcebus murinus. Biochim. Biophys. Acta Gene Regul. Mech. 1863, 194473. https://doi.org/10.1016/j.bbagrm.2019.194473

Hampton, M., Melvin, R.G., Andrews, M.T., 2013. Transcriptomic analysis of brown adipose tissue across the physiological extremes of natural hibernation. PLoS One 8, 1-12. https://doi.org/10.1371/journal.pone.0085157

Huang, W., Liao, C.C., Han, Y., Lv, J., Lei, M., Li, Y., Lv, Q., Dong, D., Zhang, S., Pan, Y.H., Luo, J., 
2020. Co-activation of Akt, Nrf2, and NF-kB signals under UPRER in torpid Myotis ricketti bats for survival. Commun. Biol. 3, 1-14. https://doi.org/10.1038/s42003-020-01378-2

Iadevaia, V., Gerber, A., 2015. Combinatorial control of mRNA fates by RNA-binding poteins and noncoding RNAs. Biomolecules 5, 2207-2222. https://doi.org/10.3390/biom5042207

Jo, M.H., Shin, S., Jung, S.R., Kim, E., Song, J.J., Hohng, S., 2015. Human argonaute 2 has diverse reaction pathways on target RNAs. Mol. Cell 59, 117-124. https://doi.org/10.1016/j.molcel.2015.04.027

Jung, E., Seong, Y., Jeon, B., Kwon, Y.-S., Song, H., 2018. MicroRNAs of miR-17-92 cluster increase gene expression by targeting mRNA-destabilization pathways. Biochim. Biophys. Acta - Gene Regul. Mech. 1861, 603-612. https://doi.org/10.1016/j.bbagrm.2018.06.003

Kalvari, I., Nawrocki, E.P., Argasinska, J., Quinones-Olvera, N., Finn, R.D., Bateman, A., Petrov, A.I., 2018. Non-coding RNA analysis using the Rfam database. Curr. Protoc. Bioinforma. 62, e51. https://doi.org/10.1002/cpbi.51

Knight, J.E., Narus, E.N., Martin, S.L., Jacobson, A., Barnes, B.M., Boyer, B.B., 2000. mRNA stability and polysome loss in hibernating Arctic ground squirrels (Spermophilus parryii). Mol. Cell. Biol. 20, 6374-6379. https://doi.org/10.1128/MCB.20.17.6374-6379.2000

Kozomara, A., Birgaoanu, M., Griffiths-Jones, S., 2019. MiRBase: From microRNA sequences to function. Nucleic Acids Res. 47, D155-D162. https://doi.org/10.1093/nar/gky1141

Kusuyama, J., Komorizono, A., Bandow, K., Ohnishi, T., Matsuguchi, T., 2016. CXCL3 positively regulates adipogenic differentiation. J. Lipid Res. 57, 1806-1820. https://doi.org/10.1194/jlr.M067207

Law, C.W., Chen, Y., Shi, W., Smyth, G.K., 2014. voom: precision weights unlock linear model analysis tools for RNA-seq read counts. Genome Biol. 15, R29. https://doi.org/10.1186/gb-2014-15-2-r29

Lee, M.-W., Lee, M., Oh, K., 2019. Adipose tissue-derived signatures for obesity and type 2 diabetes: adipokines, batokines and microRNAs. J. Clin. Med. 8, 854. https://doi.org/10.3390/jcm8060854

Li, H., Handsaker, B., Wysoker, A., Fennell, T., Ruan, J., Homer, N., Marth, G., Abecasis, G., Durbin, R., 2009. The sequence alignment/map format and SAMtools. Bioinformatics 25, 2078-2079. https://doi.org/10.1093/bioinformatics/btp352

Liu, W., Bi, P., Shan, T., Yang, X., Yin, H., Wang, Y.X., Liu, N., Rudnicki, M.A., Kuang, S., 2013. miR133a regulates adipocyte browning in vivo. PLoS Genet. 9. https://doi.org/10.1371/journal.pgen.1003626

Logan, Samantha M., Storey, K.B., 2021. Inflammasome signaling could be used to sense and respond to endogenous damage in brown but not white adipose tissue of a hibernating ground squirrel. Dev. Comp. Immunol. 114, 103819. https://doi.org/10.1016/j.dci.2020.103819

Logan, S.M., Storey, K.B., 2021. MicroRNA expression patterns in the brown fat of hibernating 13-lined ground squirrels. Genomics 113. https://doi.org/10.1016/j.ygeno.2021.01.017

Logan, S.M., Storey, K.B., 2018. Pro-inflammatory AGE-RAGE signaling is activated during arousal from hibernation in ground squirrel adipose. PeerJ 6, e4911. https://doi.org/10.7717/peerj.4911

Logan, S.M., Wu, C., Storey, K.B., 2019. The squirrel with the lagging eIF2: Global suppression of protein synthesis during torpor. Comp. Biochem. Physiol. Part A Mol. Integr. Physiol. 227, 161-171. https://doi.org/10.1016/j.cbpa.2018.10.014

Lou, N., Lennard Richard, M.L., Yu, J., Kindy, M., Zhang, X.K., 2017. The Fli-1 transcription factor is a critical regulator for controlling the expression of chemokine C-X-C motif ligand 2 (CXCL2). Mol. Immunol. 81, 59-66. https://doi.org/10.1016/j.molimm.2016.11.007 
Luo, W., Pant, G., Bhavnasi, Y.K., Blanchard, S.G., Brouwer, C., 2017. Pathview Web: user friendly pathway visualization and data integration. Nucleic Acids Res. 45, W501-W508. https://doi.org/10.1093/nar/gkx372

Mamady, H., Storey, K.B., 2008. Coping with the stress: Expression of ATF4, ATF6, and downstream targets in organs of hibernating ground squirrels. Arch. Biochem. Biophys. 477, 77-85. https://doi.org/10.1016/j.abb.2008.05.006

Margolis, L.M., Rivas, D.A., Pasiakos, S.M., McClung, J.P., Ceglia, L., Fielding, R.A., 2017. Upregulation of circulating myomiR following short-term energy restriction is inversely associated with whole body protein synthesis. Am. J. Physiol. Integr. Comp. Physiol. 313, R298-R304.

https://doi.org/10.1152/ajpregu.00054.2017

Markowski, D.N., Thies, H.W., Gottlieb, A., Wenk, H., Wischnewsky, M., Bullerdiek, J., 2013. HMGA2 expression in white adipose tissue linking cellular senescence with diabetes. Genes Nutr. 8, 449-456. https://doi.org/10.1007/s12263-013-0354-6

Maurizi, G., Della Guardia, L., Maurizi, A., Poloni, A., 2018. Adipocytes properties and crosstalk with immune system in obesity-related inflammation. J. Cell. Physiol. 233, 88-97. https://doi.org/10.1002/jcp.25855

McMullen, D.C., Hallenbeck, J.M., 2010. Regulation of Akt during torpor in the hibernating ground squirrel, Ictidomys tridecemlineatus. J. Comp. Physiol. B Biochem. Syst. Environ. Physiol. 180, $927-$ 934. https://doi.org/10.1007/s00360-010-0468-8

Miyake, S.-I., Wakita, H., Bernstock, J.D., Castri, P., Ruetzler, C., Miyake, J., Lee, Y., Hallenbeck, J.M., 2015. Hypophosphorylation of ribosomal protein S6 is a molecular mechanism underlying ischemic tolerance induced by either hibernation or preconditioning. J. Neurochem. 135, 943-957. https://doi.org/10.1111/jnc. 13368

Moon, T.W., 1978. Enzymes of heterotherms: LDH of hibernating and normothermic little brown bats, Myotis lucifugus. Comp. Biochem. Physiol. -- Part B Biochem. 59, 183-190. https://doi.org/10.1016/0305-0491(78)90242-0

Murakami, M., Kamimura, D., Hirano, T., 2019. Pleiotropy and specificity: insights from the interleukin 6 family of cytokines. Immunity 50, 812-831. https://doi.org/10.1016/j.immuni.2019.03.027

Ni, B., Farrar, J.S., Vaitkus, J.A., Celi, F.S., 2015. Metabolic Effects of FGF-21: Thermoregulation and Beyond. Front. Endocrinol. (Lausanne). 6, 1-7. https://doi.org/10.3389/fendo.2015.00148

Pivorun, E.B., Sinnamon, W.B., 1981. Blood coagulation studies in normothermic, hibernating, and aroused Spermophilus franklini. Cryobiology 18, 515-520. https://doi.org/10.1016/00112240(81)90212-1

Quinn, T.P., Erb, I., Richardson, M.F., Crowley, T.M., 2018. Understanding sequencing data as compositions: An outlook and review. Bioinformatics 34, 2870-2878. https://doi.org/10.1093/bioinformatics/bty175

Rolfe, D.F., Brown, G.C., 1997. Cellular energy utilization and molecular origin of standard metabolic rate in mammals. Physiol. Rev. 77, 731-758.

Rouble, A.N., Hefler, J., Mamady, H., Storey, K.B., Tessier, S.N., 2013. Anti-apoptotic signaling as a cytoprotective mechanism in mammalian hibernation. PeerJ 1, e29. https://doi.org/10.7717/peerj.29

Rouble, A.N., Tessier, S.N., Storey, K.B., 2014. Characterization of adipocyte stress response pathways during hibernation in 13-lined ground squirrels. Mol. Cell. Biochem. 393, 271-282. https://doi.org/10.1007/s11010-014-2070-y

Ruberto, A.A., 2015. Regulation of lactate dehydrogenase and glycerol-3-phosphate dehydrogenase in mammalian hibernation. 
Sai lakshmi, S., Agrawal, S., 2008. piRNABank: A web resource on classified and clustered Piwiinteracting RNAs. Nucleic Acids Res. 36. https://doi.org/10.1093/nar/gkm696

Schuster, N., Krieglstein, K., 2002. Mechanisms of TGF-beta-mediated apoptosis. Cell Tissue Res. 307, 114. https://doi.org/10.1007/s00441-001-0479-6

Shan, Z.-X., Lin, Q.-X., Deng, C.-Y., Zhu, J.-N., Mai, L.-P., Liu, J.-L., Fu, Y.-H., Liu, X.-Y., Li, Y.-X., Zhang, Y.-Y., Lin, S.-G., Yu, X.-Y., 2010. miR-1/miR-206 regulate Hsp60 expression contributing to glucose-mediated apoptosis in cardiomyocytes. FEBS Lett. 584, 3592-3600. https://doi.org/10.1016/j.febslet.2010.07.027

Stahlhut, C., Suarez, Y., Lu, J., Mishima, Y., Giraldez, A.J., 2012. miR-1 and miR-206 regulate angiogenesis by modulating VegfA expression in zebrafish. Development 139, 4356-4365. https://doi.org/10.1242/dev.083774

Storey, K.B., 2010. Out cold: biochemical regulation of mammalian hibernation - A mini-review. Gerontology 56, 220-230. https://doi.org/10.1159/000228829

Storey, K.B., 1987. Regulation of liver metabolism by enzyme phosphorylation during mammalian hibernation. J. Biol. Chem. 262, 1670-1673.

Tessier, S.N., Wu, C.-W., Storey, K.B., 2019. Molecular control of protein synthesis, glucose metabolism, and apoptosis in the brain of hibernating 13-lined ground squirrels. Biochem. Cell Biol. 1-29. https://doi.org/10.1139/bcb-2018-0256

Townsend, K., Tseng, Y.-H., 2012. Brown adipose tissue: Recent insights into development, metabolic function and therapeutic potential. Adipocyte 1,13-24. https://doi.org/10.4161/adip.18951

Trajkovski, M., Ahmed, K., Esau, C.C., Stoffel, M., 2012. MyomiR-133 regulates brown fat differentiation through Prdm16. Nat. Cell Biol. 14, 1330-1335. https://doi.org/10.1038/ncb2612

Trayhurn, P., 2018. Brown adipose tissue - A therapeutic target in obesity? Front. Physiol. 9. https://doi.org/10.3389/fphys.2018.01672

van Breukelen, F., Martin, S.L., 2001. Translational initiation is uncoupled from elongation at 18 degrees C during mammalian hibernation. Am. J. Physiol. Regul. Integr. Comp. Physiol. 281, R1374-R1379.

Walden, T.B., Timmons, J.A., Keller, P., Nedergaard, J., Cannon, B., 2009. Distinct expression of musclespecific microRNAs (myomirs) in brown adipocytes. J. Cell. Physiol. 218, 444-449. https://doi.org/10.1002/jcp.21621

Wang, L.C.H., Wolowyk, M.W., 1988. Torpor in mammals and birds. Can. J. Zool. 66, 133-137. https://doi.org/10.1139/z88-017

Wang, X., Chang, L., Wang, H., Su, A., Wu, Z., 2017. Dcp1a and GW182 induce distinct cellular aggregates and have different effects on microRNA pathway. DNA Cell Biol. 36, 565-570. https://doi.org/10.1089/dna.2017.3633

Ward, J.H., 1963. Hierarchical grouping to optimize an objective function. J. Am. Stat. Assoc. 58, 236. https://doi.org/10.2307/2282967

Whitten, B.K., Klain, G.J., 1968. Protein metabolism in hepatic tissue of hibernating and arousing ground squirrels. Am. J. Physiol. 214, 1360. https://doi.org/10.1152/ajplegacy.1968.214.6.1360

Wu, C.-W., Biggar, K.K., Storey, K.B., 2014. Expression profiling and structural characterization of microRNAs in adipose tissues of hibernating ground squirrels. Genomics. Proteomics Bioinformatics 12, 284-291. https://doi.org/10.1016/j.gpb.2014.08.003

Wu, C.-W., Storey, K.B., 2016. Life in the cold: links between mammalian hibernation and longevity. Biomol. Concepts 7, 41-52. https://doi.org/10.1515/bmc-2015-0032 
Yuan, L., Geiser, F., Lin, B., Sun, H., Chen, J., Zhang, S., 2015. Down but not out: the role of microRNAs in hibernating bats. PLoS One 10, e0135064. https://doi.org/10.1371/journal.pone.0135064

Zhang, J., Hadj-Moussa, H., Storey, K.B., 2020. Marine periwinkle stress-responsive microRNAs: A potential factor to reflect anoxia and freezing survival adaptations. Genomics 112, 4385-4398. https://doi.org/10.1016/j.ygeno.2020.07.036

Zhang, J., Hadj-Moussa, H., Storey, K.B., 2016. Current progress of high-throughput microRNA differential expression analysis and random forest gene selection for model and non-model systems: an R implementation. J. Integr. Bioinform. 13, 306. https://doi.org/10.2390/biecoll-jib-2016-306

Zhang, J., Storey, K.B., 2018. RBiomirGS: An all-in-one miRNA gene set analysis solution featuring target mRNA mapping and expression profile integration. PeerJ 2018, 1-17. https://doi.org/10.7717/peerj.4262

Zhang, J., Storey, K.B., 2016. RBioplot: an easy-to-use R pipeline for automated statistical analysis and data visualization in molecular biology and biochemistry. PeerJ 4, e2436. https://doi.org/10.7717/peerj.2436

Zhang, Z., Jiang, H., Li, X., Chen, X., Huang, Y., 2020. MiR-92a regulates brown adipocytes differentiation, mitochondrial oxidative respiration, and heat generation by targeting SMAD7. J. Cell. Biochem. 121, 3825-3836. https://doi.org/10.1002/jcb.29539 


\subsection{Tables}

Table 4.1: Summary of Pearson correlation coefficients, $p$-values and FDR-adjusted p-values for positively correlated miRNAs of interest, miRNAs 1-3p, 206, 133a-3p, and 361-3p

$\begin{array}{lllll}\text { miRNA 1 } & \text { miRNA 2 } & \text { Coefficient } & \text { p-value } & \text { q-value } \\ \text { hsa-miR-1-3p } & \text { hsa-miR-361-3p } & 0.999975 & 2.49 \mathrm{E}-05 & 0.035465 \\ \text { hsa-miR-133a-3p } & \text { hsa-miR-206 } & 0.999305 & 0.000695 & 0.27321 \\ \text { hsa-miR-1-3p } & \text { hsa-miR-206 } & 0.975437 & 0.024563 & 0.56004 \\ \text { hsa-miR-206 } & \text { hsa-miR-361-3p } & 0.974078 & 0.025922 & 0.562616 \\ \text { hsa-miR-1-3p } & \text { hsa-miR-133a-3p } & 0.970719 & 0.029281 & 0.568761 \\ \text { hsa-miR-133a-3p } & \text { hsa-miR-361-3p } & 0.969371 & 0.030629 & 0.568761\end{array}$




\subsection{Figures}
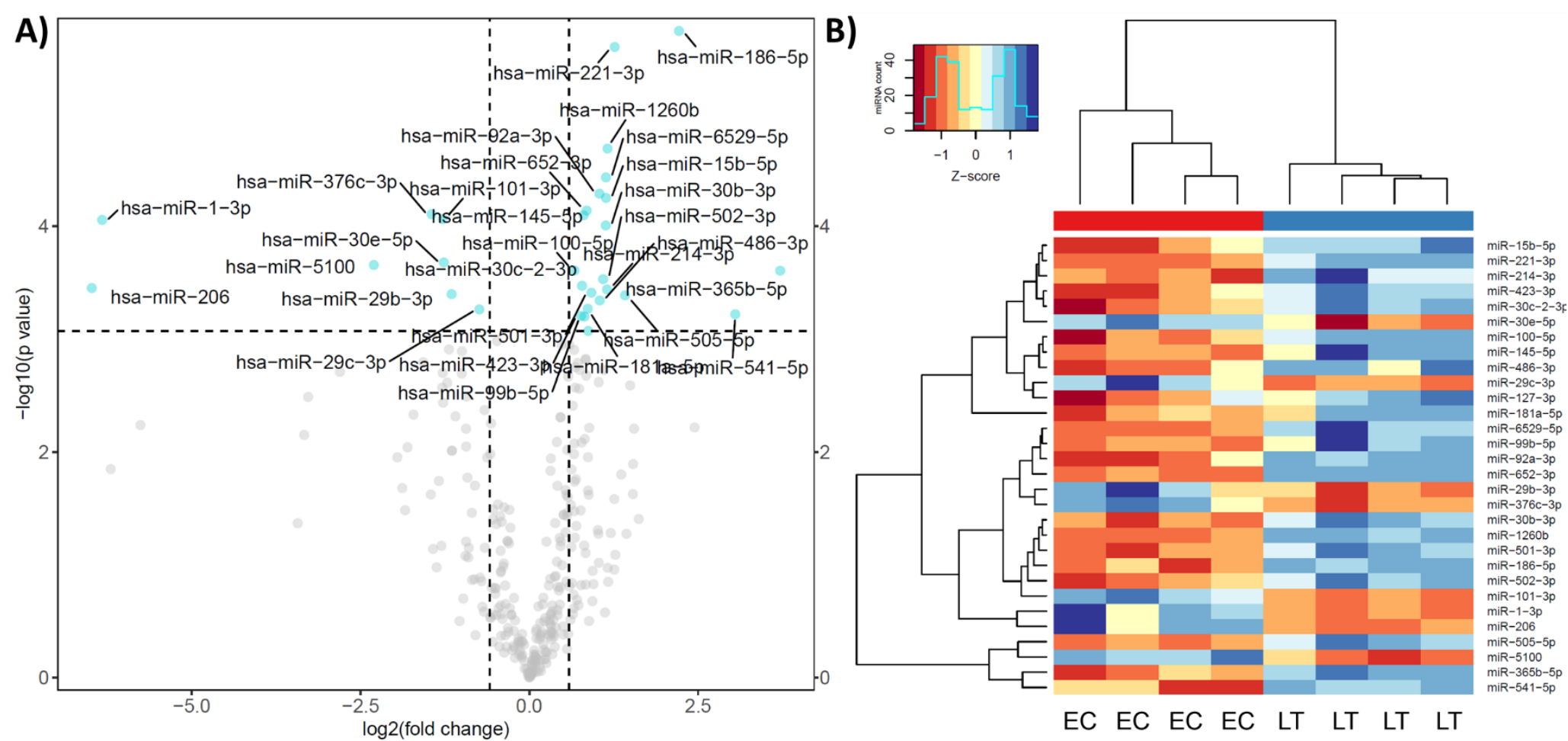

Figure 4.1: Differential microRNA abundance in 13-lined ground squirrel BAT considering fold changes $>1.5$ and q $<0.01$. A) Volcano plot showing logarithmically transformed ( $\log 2)$ fold-change in miRNA expression with $\log 10$ transformed q-values, where dotted lines show the thresholds of statistical significance for each metric. B) Supervised cluster analysis of the DE miRNA species in control (sample numbers 1-4) and torpor samples (samples 5-8). Blue represents high miRNA read counts, and red represents low miRNA read counts. On the y-axis, a dendrogram shows miRNAs clustered based on similarity in expression patterns. Dendrograms also indicate which EC samples (Red bar) or LT samples (Blue bar) have similar expression patterns. From left to right, sample numbers are as follows: 4, 2, 3, 1, 8, 6,7 and 5. 


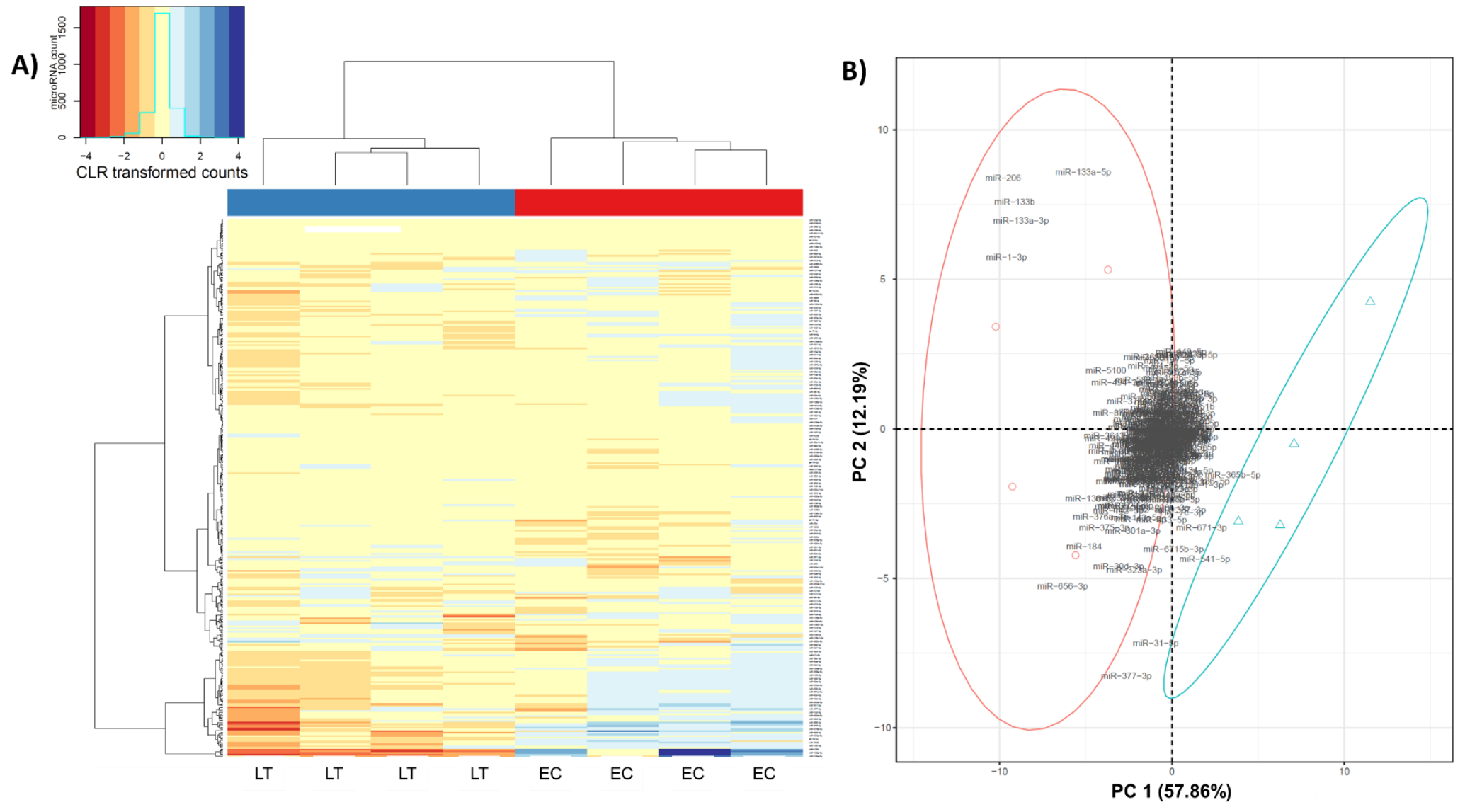

Figure 4.2: Analysis of trends in the complete miRNA dataset. A) Heatmap of the unsupervised cluster analysis, based on miRNA expression patterns from EC and LT (Torpor) in 13-lined ground squirrel BAT. Dendrogram shows clustering of EC and LT samples, respectively (seafoam green and pink bars). Dendrograms on the Y-axis show miRNAs that cluster by similar expression patterns. Brown shading represents low miRNA counts and green shading represent high miRNA counts. B) Principal component analysis of the EC (pink ellipse and circles representing biological replicates) and LT (blue ellipse and triangles representing biological replicates) samples shows significant separation. 


\section{EC}

A)

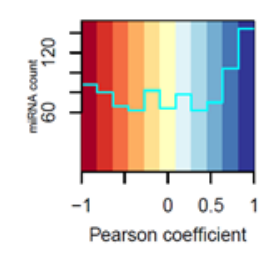

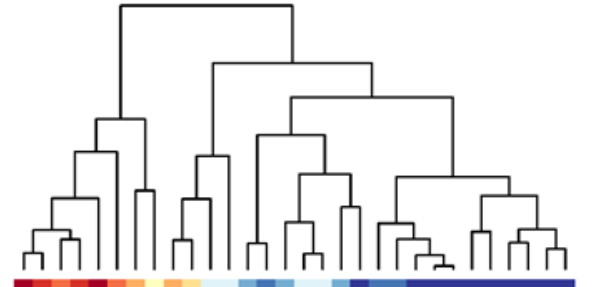

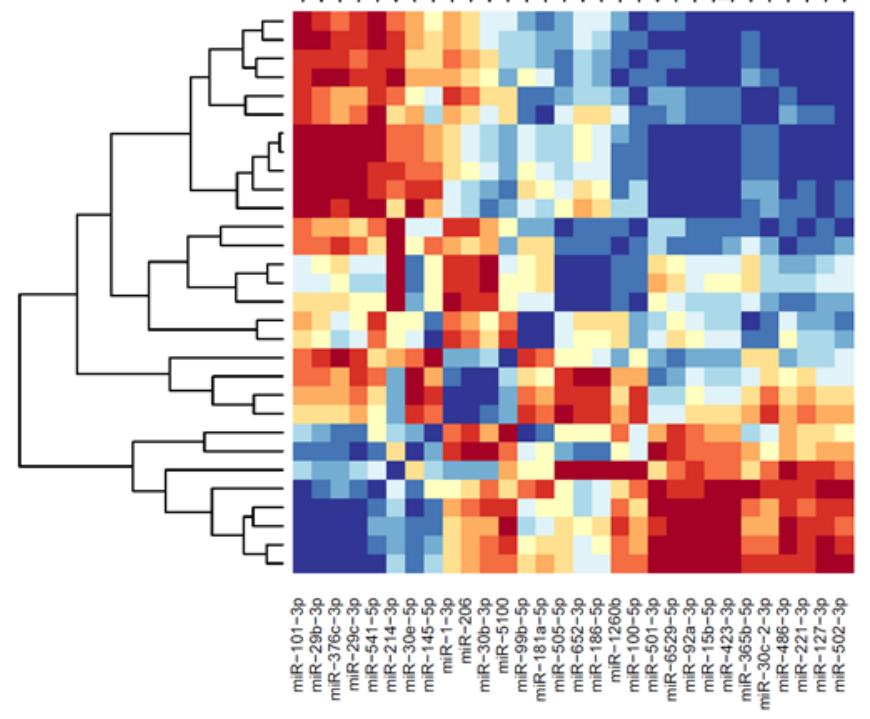

B)

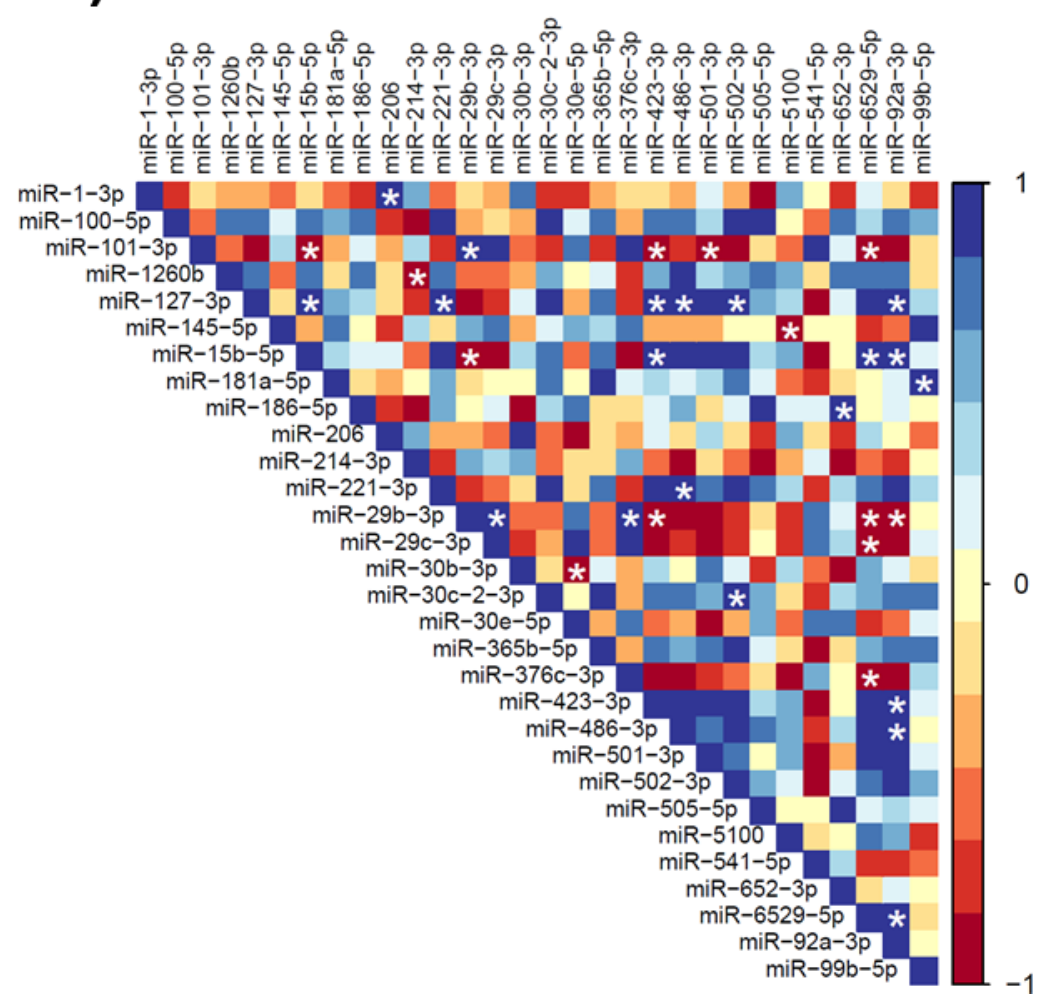

Figure 4.3: Supervised Pearson correlation heatmap (A) and correlation plot (B) of differentially abundant microRNAs in BAT from 13-lined ground squirrel sampled during control (EC) conditions. White stars on the correlation plots (B) indicate statistical significance $(\mathrm{q}<0.01)$, and dark blue is used to depict miRNAs that have congruent abundance patterns and dark red signifies opposite miRNA abundance patterns. 


\section{LT}

A)
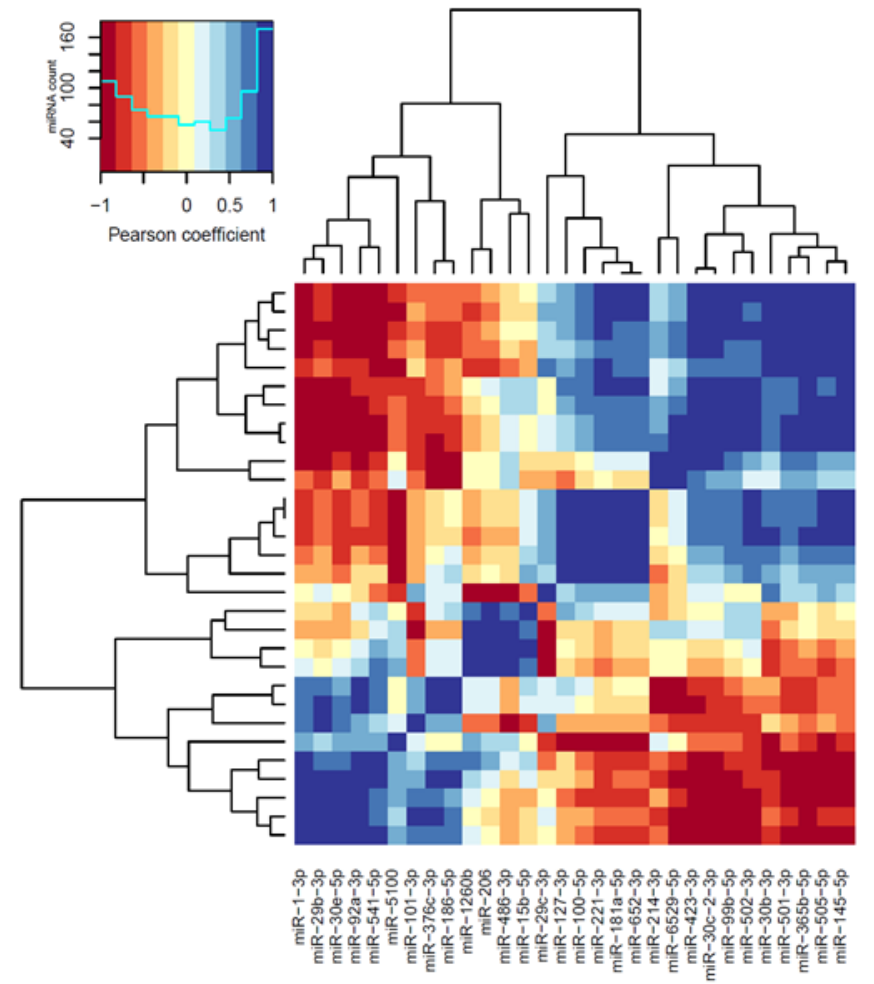

B)

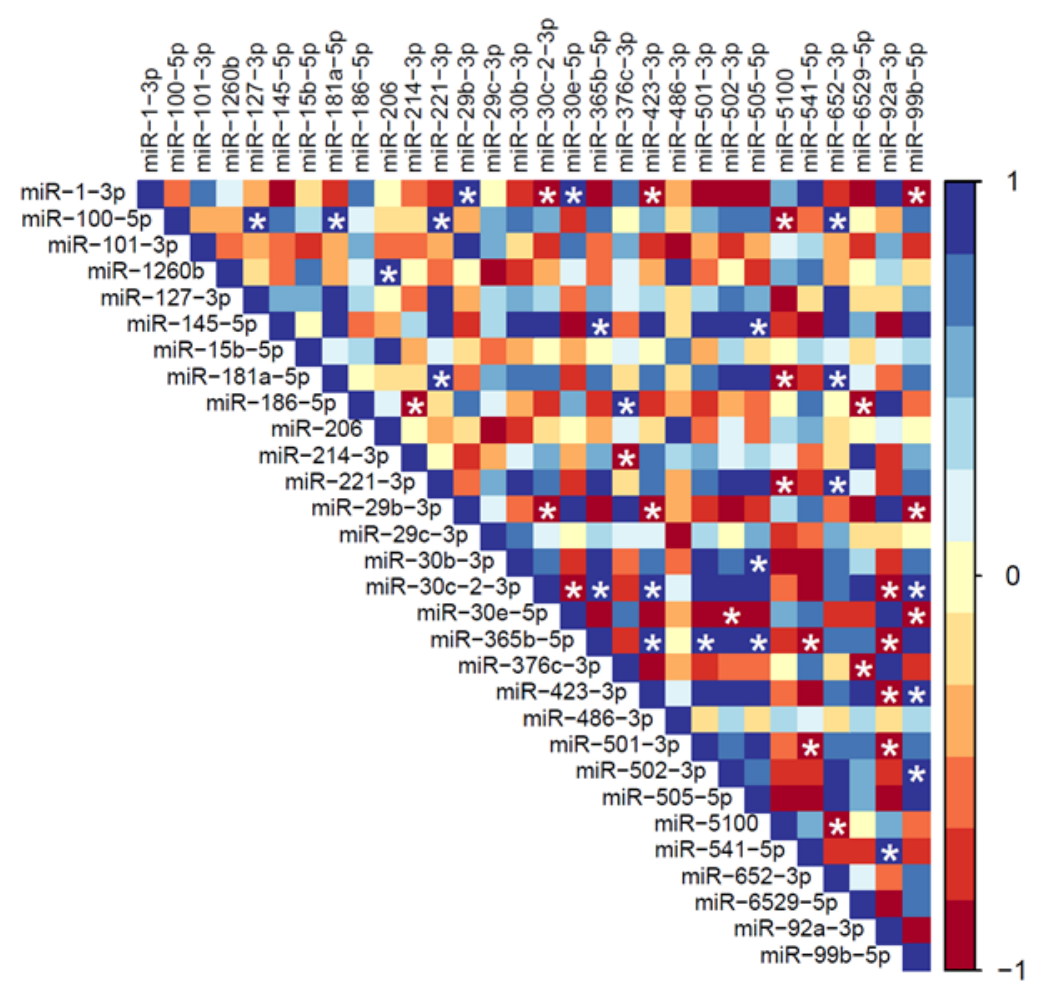

Figure 4.4: Supervised Pearson correlation heatmap (A) and correlation plot (B) of differentially abundant microRNAs in BAT from 13-lined ground squirrel sampled during torpor (LT) conditions. White stars on the correlation plot (B) indicate statistical significance $(\mathrm{q}<0.01)$, and dark blue is used to depict miRNAs that have congruent abundance patterns and dark red signifies opposite miRNA abundance patterns. 

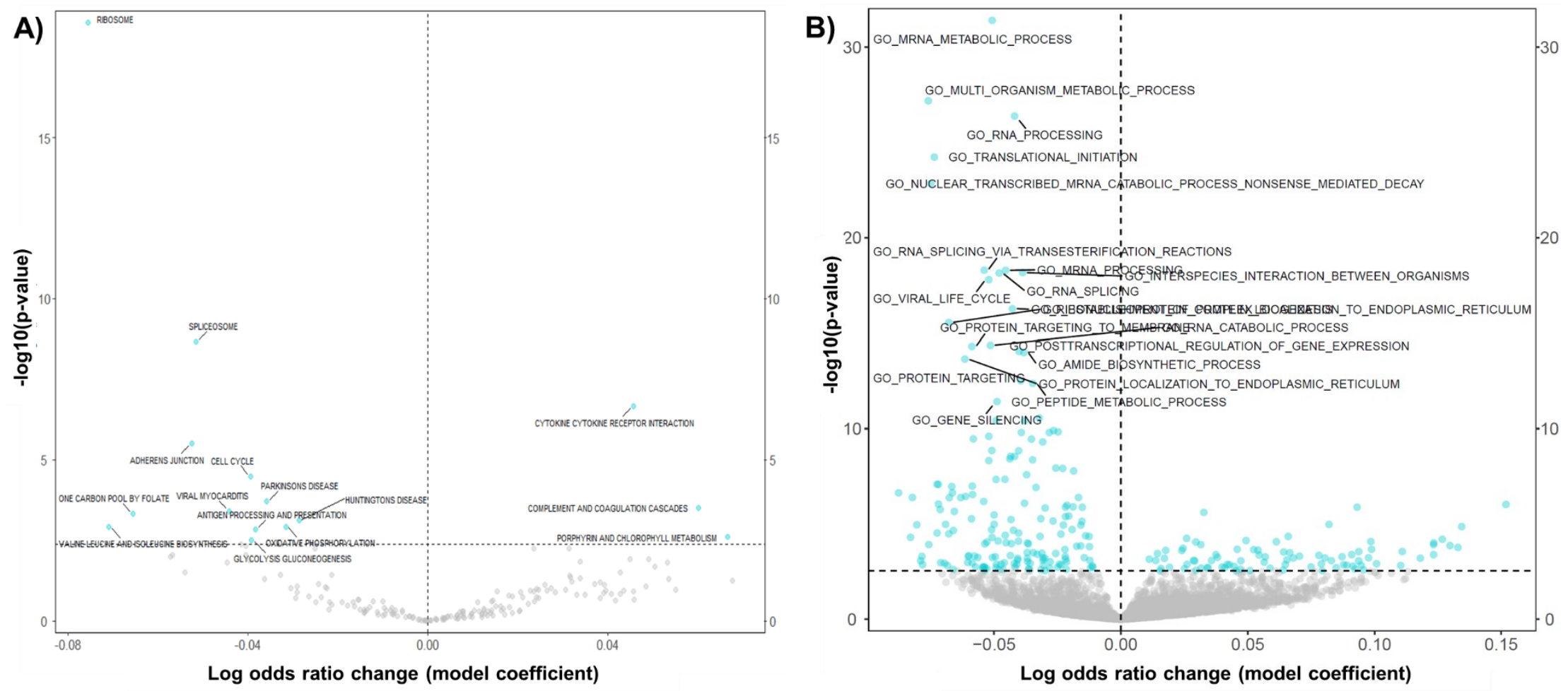

Figure 4.5: Gene set analysis of global miRNA expression patterns. Volcano plots show log2 transformed model coefficients and their distribution with respect to the $\log 10$ p-value. A) significantly enriched ( $<<0.05)$ KEGG Pathways, (B) GO biological processes. Negative coefficients indicate pathways/processes/functions that are significantly downregulated by miRNAs during LT and positive coefficients are less downregulated by miRNAs during LT. 


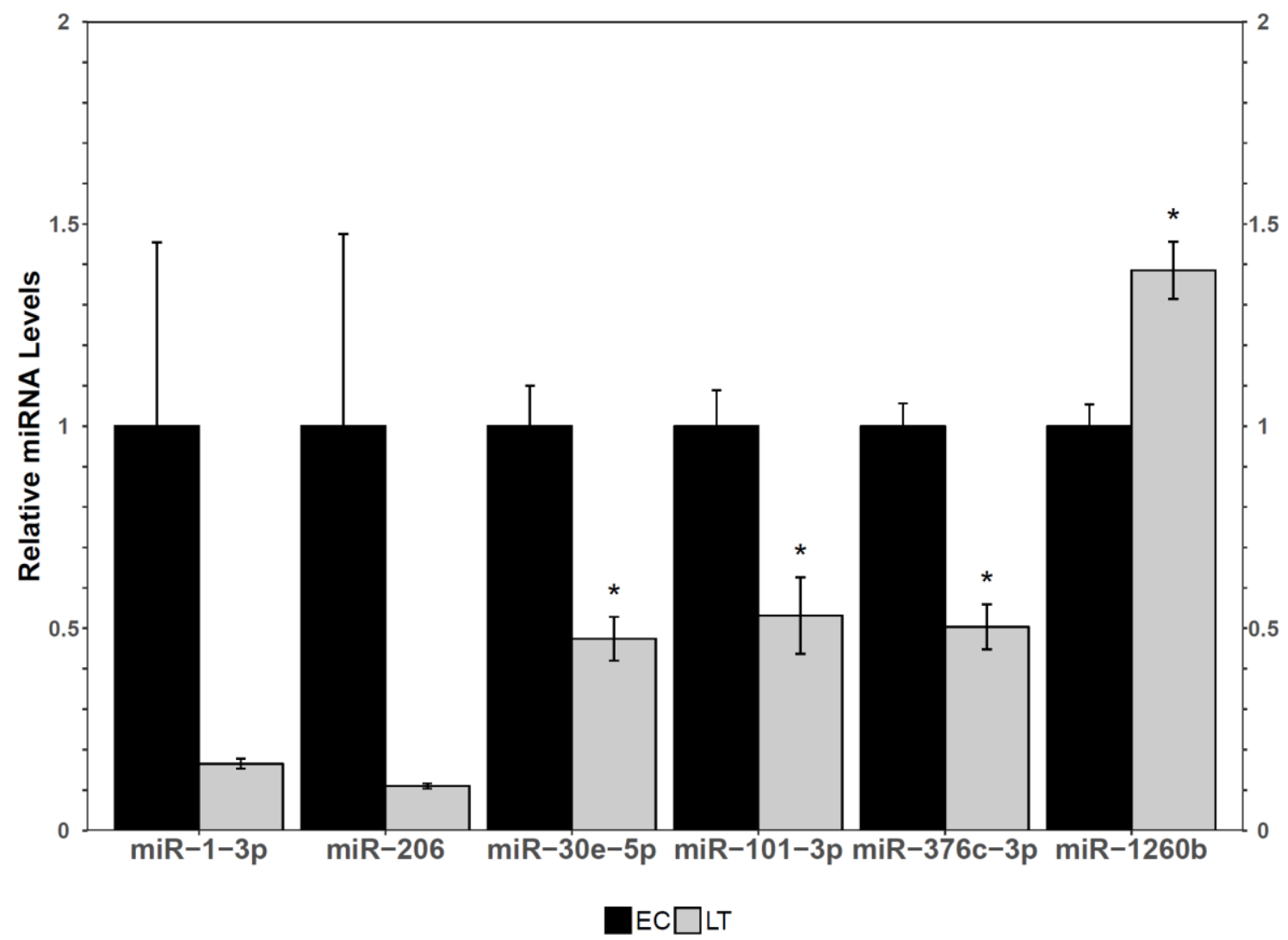

Figure 4.6: RT-qPCR validation of the expression patterns of miRNAs miR-1-3p, miR-206, miR-30e-5p, miR-101-3p, miR-376c-3p, miR-1260b. Snord-68 was used as the reference gene. Biological replicates $(n=4)$ were used for all miRNAs except an $n=3$ was used for EC samples in the analysis of all miRNAs except miR-1-3p and miR-206. Expression patterns were deemed statistically significant if a Student's t-test resulted in $\mathrm{p}<0.05$. 


\subsection{Supplemental Tables}

Data available in: (S.M. Logan and Storey, 2021).

Supplemental Table 4.1: Differential expression of miRNAs using $\mathrm{q}<0.05$ as the threshold for significance.

Supplemental Table 4.2: Highly significant Pearson correlation clusters of miRNAs $(\mathrm{q}<0.01)$ for EC and LT BAT samples.

Supplemental Table 4.3: Significant Pearson correlation clusters of miRNAs $(q<0.5)$ for EC and LT samples.

Supplemental Table 4.4: Experimentally validated interactions between all miRNAs and mRNAs.

Supplemental Table 4.5: KEGG, GO biological process, and GO molecular function analyses.

Supplemental Table 4.6: Random forest feature selection results.

Supplemental Table 4.7: PLS-DA Permutation test results, showing RMSEP values for all 999 iterations for both EC and LT comparisons.

Supplemental Table 4.8: Primers used in RT-qPCR analyses of miRNAs. 


\subsection{Supplemental Figures}

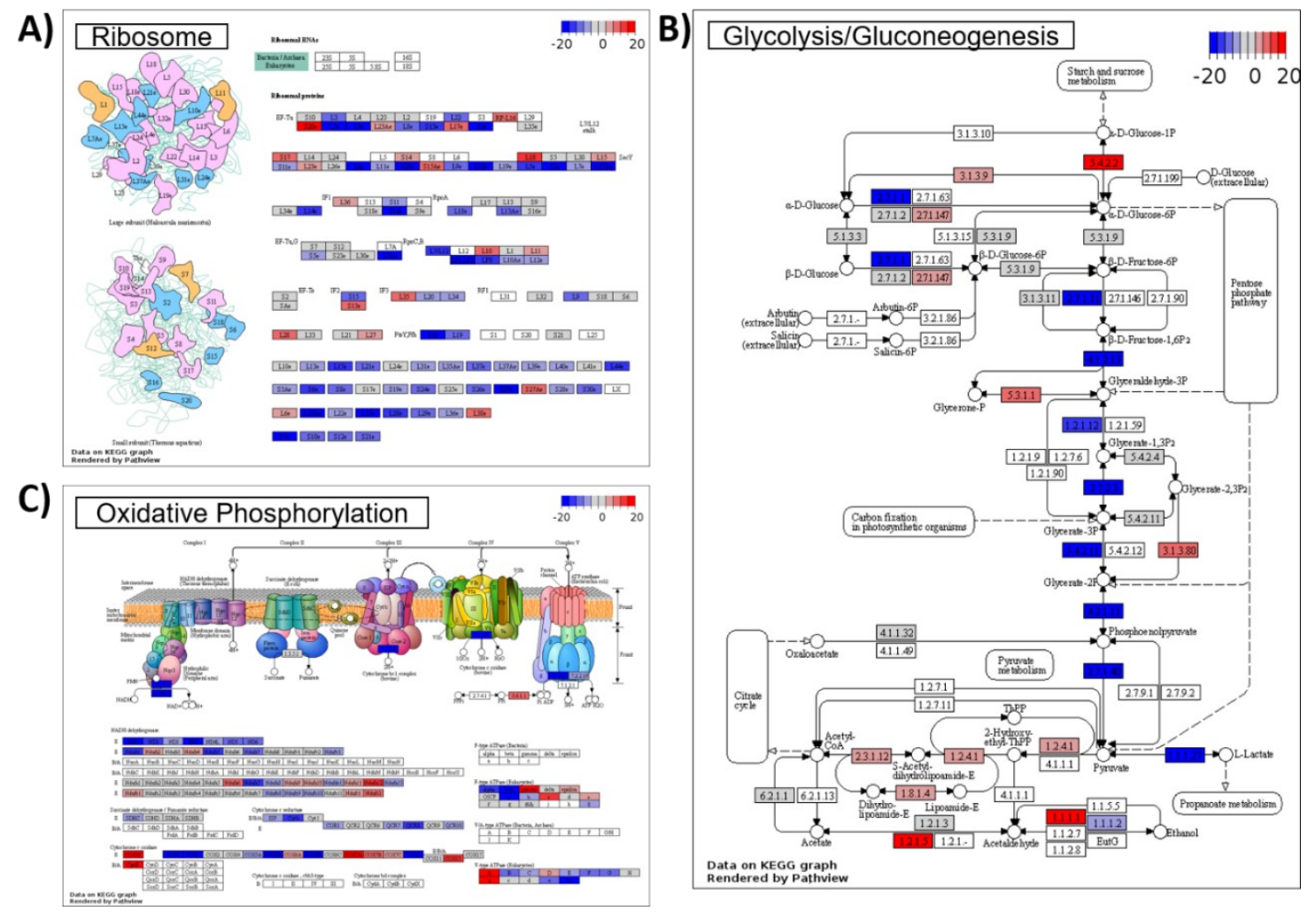

Supplemental Figure 4.1: Pathview analysis of significantly enriched and miRNA-inhibited pathways during LT. Highlighted KEGG pathways include A) ribosome, B) glycolysis and gluconeogenesis and C) oxidative phosphorylation. Genes in blue indicate genes that are likely downregulated by miRNAs, and genes in red are likely uninhibited by miRNAs. 


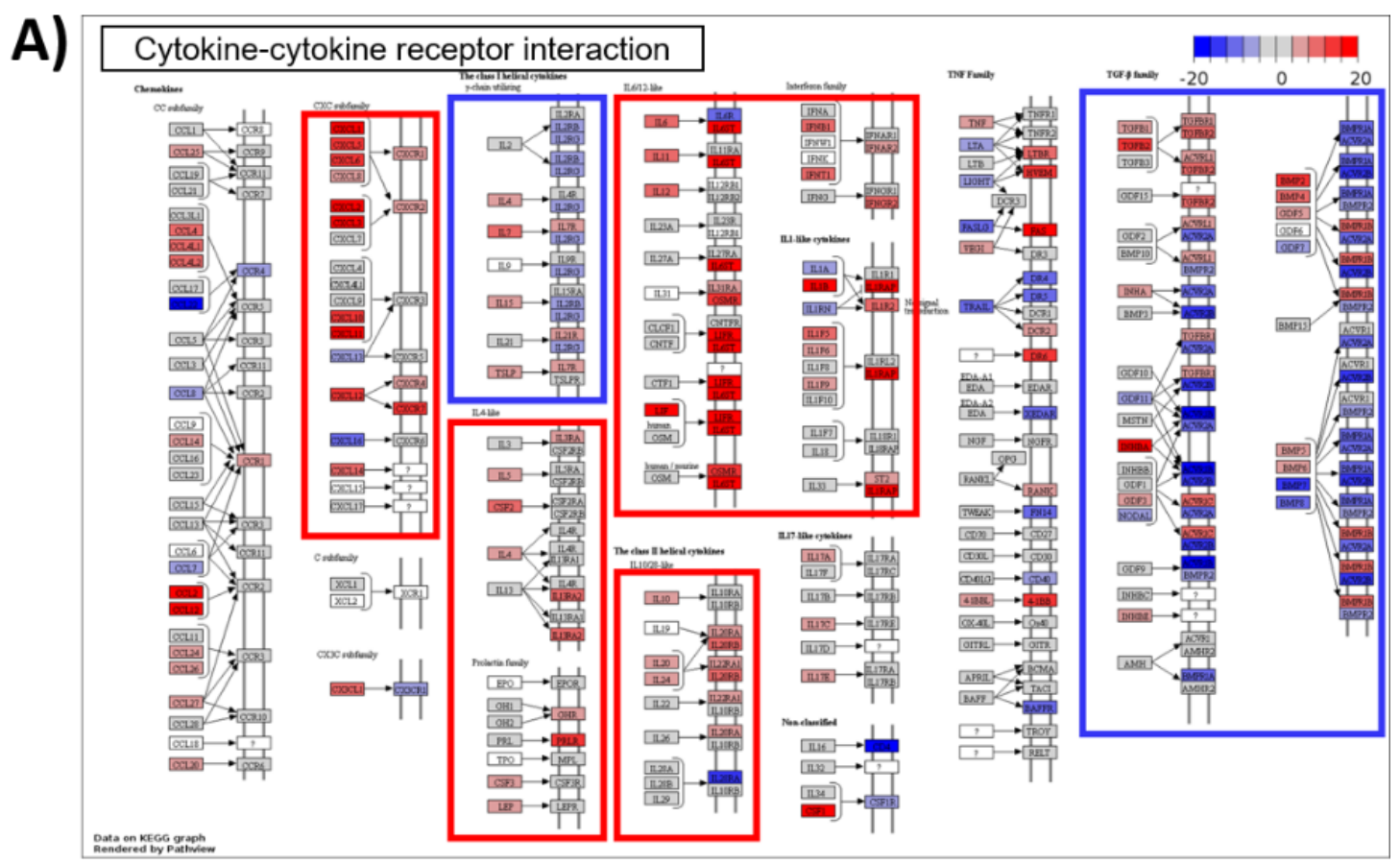

B)

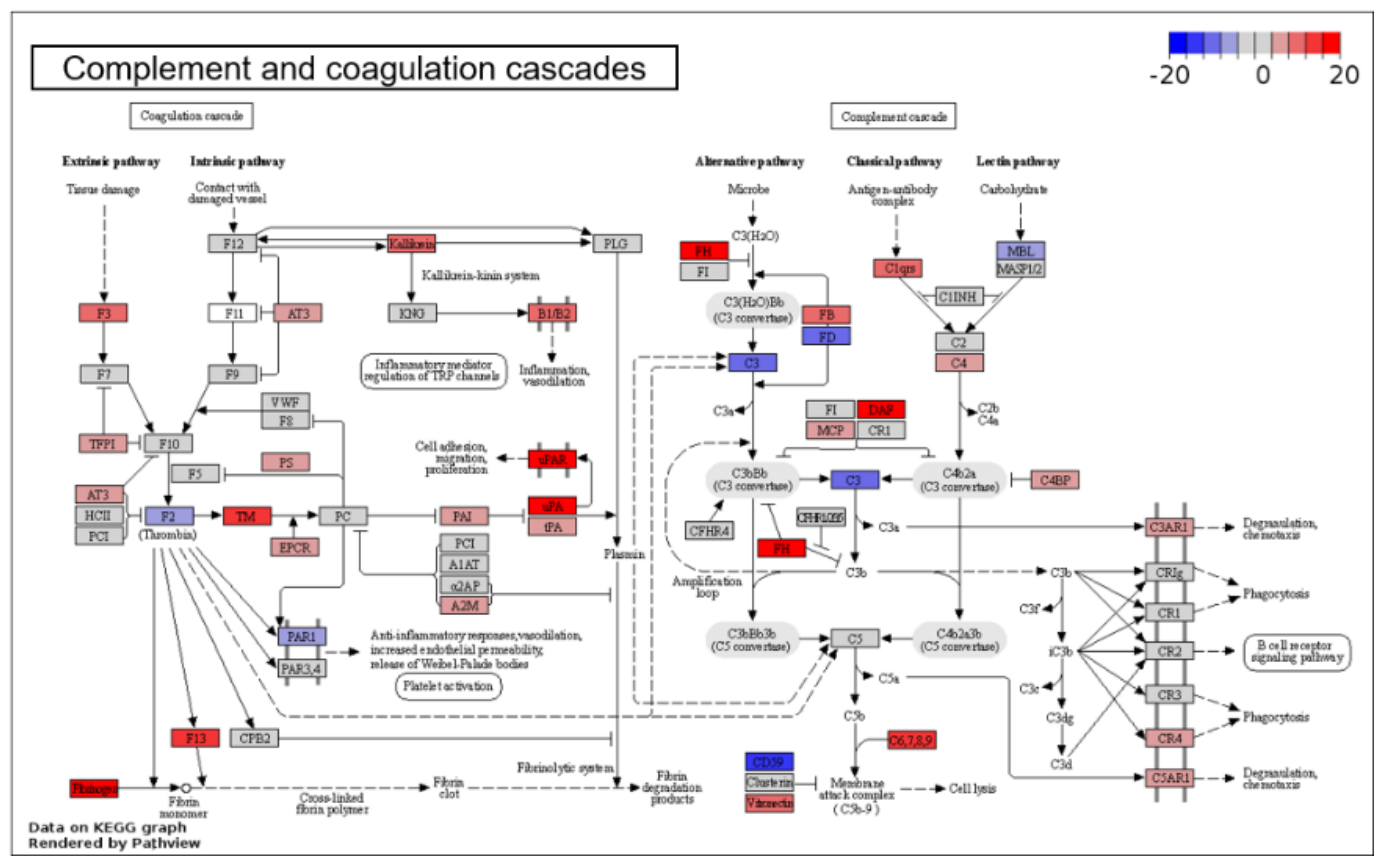

Supplemental Figure 4.2: Pathview analysis of significantly enriched and less inhibited KEGG pathways during torpor. Highlighted KEGG pathways include A) Cytokine-cytokine receptor interactions and B) complement and coagulation cascades. Other information as in Supplemental Figure 4.1. 

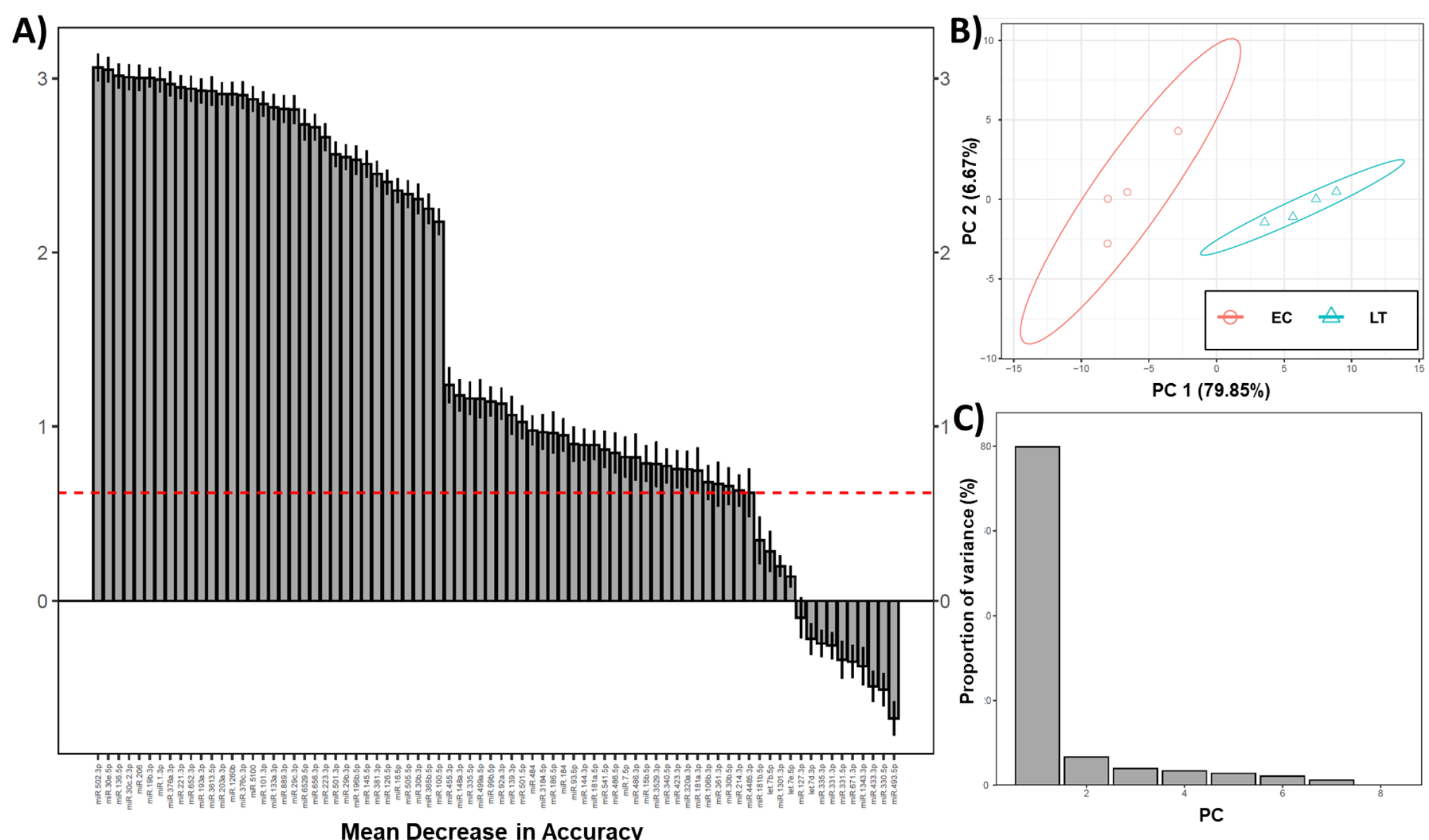

Supplemental Figure 4.3: Random forest feature selection and principal component analysis of resulting genes. A) Histogram of initial feature selection shows the variable importance of 78 miRNAs and standard error on VI. The initial selection narrowed the gene list down to 64 miRNAs. B) PCA biplot showing complete separation of EC and LT conditions using the subset of miRNAs. C) PCA Boxplot showing the variance explained by each principal component 

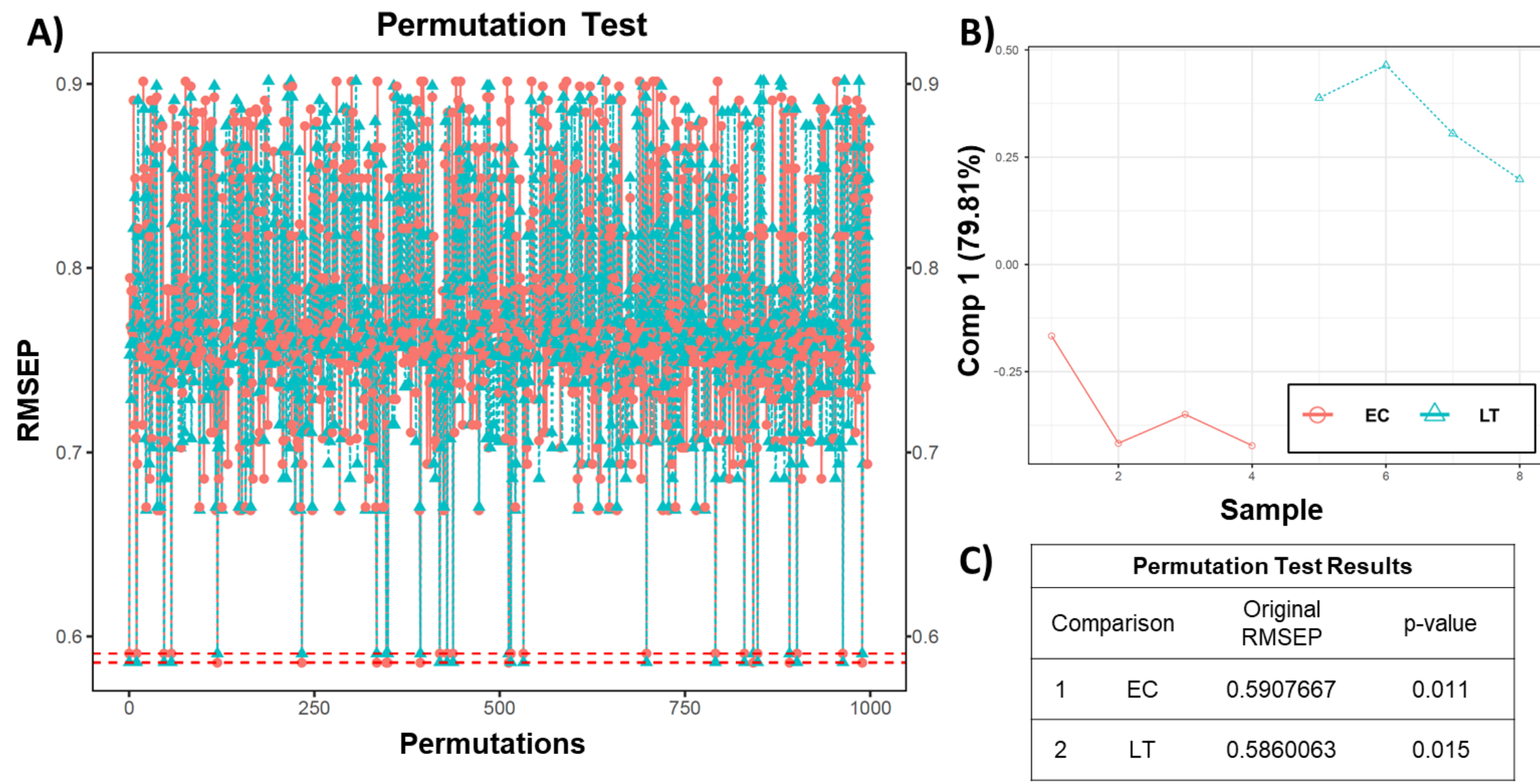

Supplemental Figure 4.4: PLS-DA model A) permutation test and b) final model using one component. C) summary of the permutation test, where $\mathrm{p}<0.05$ indicates the model can reliably classify samples as either EC or LT using the subset of 64 miRNA 
5 MicroRNA regulation of metabolically suppressed white adipose tissue in a wild fatstoring hibernator, Ictidomys tridecemlineatus 


\subsection{Abstract}

Hibernators become obese in the fall in preparation for metabolic suppression, characterized by decreased body temperature and metabolic rate. Despite characteristics of obesity such as chronically hypertrophied adipocytes and insulin/leptin resistance, ground squirrels emerge from hibernation in the spring with no apparent tissue damage or disease. Small RNA-sequencing was used to detect 314 conserved microRNAs, post-transcriptional regulators of all biological processes, in white fat from control and torpid ground squirrels, since their relative levels could inform how hibernators prevent adipocyte dysfunction. Ten miRNAs were upregulated and twenty were downregulated. Global miRNA expression patterns supported the inhibition of energy expensive pathways such as cell cycle, DNA replication, mTOR, and inflammatory signaling. DNA repair pathways were less inhibited by miRNAs during torpor. Using RT-qPCR, miR-6729 was confirmed to be upregulated during torpor. RT-qPCR and western blotting determined that p53 mRNA and protein levels did not change in torpor compared to the control. Instead, p53 was more phosphorylated during torpor, suggesting increased stability. A possible target of miR-6729 and regulator of p53 activity, mouse double minute 2 homolog (MDM2), was less abundant during torpor. These experiments identified p53 signaling as a possibly important pathway in the response to torpor and related pathways including cell cycle and DNA repair should be more thoroughly explored.

Keywords: 13-lined ground squirrel, torpor, miRNA, cell cycle, p53, inflammation, DNA repair 


\subsection{Introduction}

Nearly $40 \%$ of the American population and $27 \%$ of the Canadian population is suffering from obesity (Hales et al., 2020; Statistics Canada, 2019). Overweight, i.e. a body mass index (BMI) of 25-30, and obesity (BMI of 30 or more) are chronic diseases that can increase the risk of co-morbidities such as heart disease, diabetes, hypertension, and some cancers (Kayser and Verges, 2013). Interestingly, small mammalian hibernators like marmots and ground squirrel can double their body mass, such that adipose depots account for up to $50 \%$ of their total body weight by the time they enter their burrows for hibernation (Boyer and Barnes, 1999; Sheriff et al., 2013; Wang and Lee, 1996). Enviable by humans, hibernator body weight is lost without exercising, as the hibernation period progresses from October to late March or April (Burnett, 1914; Kisser and Goodwin, 2012).

Hibernation is a form of torpor, that is used by monotremes, marsupials, and placentals from nearly every order including Rodentia, Primates, Chiroptera, and Carnivora (Geiser, 2004). Torpor involves suppressing metabolism greatly, sometimes down to as low as $1-5 \%$ of euthermic levels, and often involves a substantial decrease in core body temperature (Storey, 2010; Wang and Lee, 1996). Lipids are used instead of carbohydrates as fuel for cellular activities because they pack tightly and produce more energy per dry weight. When hibernators like ground squirrels arouse in the spring, they almost immediately begin to forage for food and find mates. This suggests that ground squirrels do not get sick or experience severe muscle atrophy after remaining cold and immobile for months. Further, they arouse with no comorbidities associated with chronic obesity or starvation, suggesting they naturally adapt to higher fat stores and employ cellular and molecular mechanisms to prevent adipose tissue dysfunction.

As a result, it is important to study how white adipose tissue (WAT) changes its metabolic fingerprint during torpor. Identifying the mechanisms that allow hibernator WAT to maintain complete control over adiposity, energy expenditure, and endocrine activities during 
torpor is sure to inspire new treatments for adipose tissue dysfunction and inform the development of molecular and genetic weight loss technologies. Thus, conserved microRNAs (miRNAs) were measured in the WAT of euthermic in the cold room controm (EC) and late torpid (LT) 13-lined ground squirrels, one of the most studied hibernators. MiRNAs are noncoding RNA molecules 18-23 nucleotides long, that base pair by complementarity to mRNA transcripts to inhibit their translation, whether via their degradation or sequestration for future translation (Biggar and Storey, 2014). Understanding which miRNAs are differentially regulated during torpor can help identify pathways that are preferentially inhibited in metabolically suppressed animals, and which pathways are less inhibited by miRNAs. This study expands on others that have characterized the role of pre-selected miRNAs in the regulation of the hibernation phenotype in white adipose tissue of ground squirrel and allows us to compare the WAT miRNA fingerprints of ground squirrel with other hibernator species (Wu et al., 2014; Yuan et al., 2015). 


\subsection{Methods}

\subsubsection{Animals}

All animal experiments were performed as previously described (Logan et al., 2016; McMullen and Hallenbeck, 2010). Briefly, male 13-lined ground squirrels (Ictidomys tridecemlineatus) were wild captured by a United States Department of Agriculture licensed trapper (TLS Research, Bloomingdale, IL) and transported to the laboratory of Dr. J.M. Hallenback at the National Institute of Neurological Disorders and Stroke (NINDS) (NIH, Bethesda, MD). Animal housing and hibernation experimental procedures were approved by the NINDS animal care and use committee (ACUC, \#ASP 1223-05). Each male ground squirrel was housed individually in a shoebox cage at $21^{\circ} \mathrm{C}$. Each 13-lined ground squirrel was injected subcutaneously with a sensor chip (IPTT-300; Bio Medic Data Systems) while anesthetized with 5\% isoflurane. Animals were fed a standard rodent diet and water ad libitum until they gained sufficient lipid stores to enter hibernation. To facilitate a natural transition into torpor, animals were transferred to an environmental chamber at $5{ }^{\circ} \mathrm{C}$ in constant darkness. Body temperature $\left(\mathrm{T}_{\mathrm{b}}\right)$, time, and respiration rate were observed to determine sampling points. The EC ground squirrels were housed in a cold-room and had a $\mathrm{T}_{\mathrm{b}}$ of $37^{\circ} \mathrm{C}$ at the time of euthanasia. Late torpor animals that were continuously in deep torpor for at least 5 days with $\mathrm{T}_{\mathrm{b}}$ values of $5-8{ }^{\circ} \mathrm{C}$. All 13lined ground squirrels had been through torpor-arousal bouts prior to euthanasia by decapitation and tissue sampling, as previously described (McMullen and Hallenbeck, 2010). Tissue samples were shipped to Carleton University on dry ice and then stored at $-80^{\circ} \mathrm{C}$ until use.

\subsubsection{RNA isolation}

Approximately $150 \mathrm{mg}$ of frozen white adipose tissue (WAT) was collected from EC and LT ground squirrels $(\mathrm{n}=4$ per condition) and homogenized in $1 \mathrm{~mL}$ TRIzol reagent (Invitrogen; \# 15596-018) using a glass Dounce homogenizer. Following the addition of $200 \mu \mathrm{L}$ of chloroform, the samples were repeatedly inverted, and allowed to precipitate at room temperature 
for approximately $10 \mathrm{~min}$ before $15 \mathrm{~min}$ of centrifugation at $8,960 \times \mathrm{g}$, at $4{ }^{\circ} \mathrm{C}$. The supernatant containing RNA was collected and a second TRIzol extraction was performed with half as much TRIzol and chloroform. Following a second 15 min centrifugation step at $8,960 \times \mathrm{g}$ and $4{ }^{\circ} \mathrm{C}$, the supernatant was collected and the RNA within was allowed to precipitate at room temperature for $10 \mathrm{~min}$ following the addition of $500 \mu \mathrm{L}$ of isopropanol. Centrifugation at $13,870 \times \mathrm{g}$ was performed for 5 minutes and the isopropanol was decanted. The RNA pellets were washed with $70 \%$ ethanol and centrifuged briefly for $5 \mathrm{~min}$ at $5,418 \times \mathrm{g}$. The ethanol was decanted and the RNA pellets were allowed to air dry for 10-20 min before being resuspended in $30 \mu \mathrm{L}$ of autoclaved $\mathrm{ddH}_{2} \mathrm{O}$. A Take3 and a BioTek spectrophotometer was used to assess RNA concentration and purity based on 260/280 ratio before aliquoting for RNA sequencing and RTqPCR analyses.

\subsubsection{Small RNA sequencing}

Small RNA sequencing was performed by the BC Cancer Genome Sciences Centre (British Columbia, Canada) using an Illumina NextSeq500 instrument generating single-end 75 base reads, as previously described (Zhang et al., 2020). RNA was extracted in-house and then shipped to the BC Cancer Genome Sciences Centre for processing. Briefly, RNA from $n=4$ samples of WAT tissue from both EC and LT ground squirrels were reverse transcribed. Then, PCR was used with specific primers to amplify the cDNA library. The amplified library was loaded onto a $12 \%$ polyacrylamide gel and the region containing the miRNA library $(\sim 150 \mathrm{bp})$ was excised from the gel. The size-selected library was ethanol precipitated and purified. Quality control of the final library was performed using Qubit and Agilent DNA 1000 Series II assays prior to sequencing.

\subsubsection{Data processing}

All raw sequences were submitted to the SRA database and can be accessed with the following accession number (PRJNA697924). The raw reads were analyzed in a Unix shell 
environment in advance of differential gene expression analysis in the R environment, as has been previously described (Zhang et al., 2016). Initial quality of the sequences were determined with a FastQC analysis before adapters were trimmed along with an additional 6 bases from the beginning of every sequence, since a random set of bases is incorporated between the adapters and the small RNA sequences. Then, a second FastQC analysis was performed. The $3^{\text {rd }}$ hibernating WAT sample was removed for failing to achieve sufficient per base quality scores and for having no peak in the 14-27 region, unlike the other samples. WAT8 was relabeled as LT7 for selected figures.

Using Bowtie with default settings, the trimmed sequences were aligned with a library of small non-coding RNAs (rRNA, tRNA, piRNAs, snoRNAs, and snRNAs) sequences sourced from the databases Rfam and piRNABank (Kalvari et al., 2018; Sai lakshmi and Agrawal, 2008). Sequences that aligned with the negative reference library were filtered out and any sequences that did not align to the negative reference library were aligned with sequences within a library of mature miRNA sequences from all organisms, sourced from miRBase version 22 (Kozomara et al., 2019). Aligned sequences, which had seed sequence lengths of 20 and perfect alignments to the sequences in the mature miRNA reference library were used for the analyses presented herein. SAMtools was used in the UNIX shell to index each read count with its miRNA identity into the SAM format such that it could be subsequently analyzed using the R platform ( $\mathrm{Li}$ et al., 2009). MiRNAs with read counts less than 10 were not included in the analysis. The read count data from the 7 individual fat samples $(n=4 \mathrm{EC}$ and $\mathrm{n}=3 \mathrm{LT})$ were used. Normalization of RNA-seq read counts against $\log 2$ counts-per-million $(\log C P M)$ was done before voom was used to calculate read count variance for each normalized observation (Law et al., 2014; Zhang et al., 2020). 


\subsubsection{Differential gene expression, supervised and unsupervised clustering analysis}

To quantify the differential expression of miRNAs during LT relative to EC, an empirical Bayes test was performed to linearly model the miRNA expression. Then, the Ward method was used to determine the direction of expression for each miRNA (Ward, 1963). Differentially expressed miRNAs were plotted as volcano plots with p-value $<0.05$, since there were no statistically significant changes using a false discovery rate (FDR)-adjusted $p<0.05$. Fold changes greater than 1.5 , corresponding to approximately 0.58 on the $\log 2$ (fold change) scale of the volcano plots were considered statistically significant. Supervised cluster analysis, using known associations between each sample and its condition (control or torpor), was used to cluster miRNA based on their centered-log transformed read counts.

Unsupervised cluster analysis was used to determine the similarity of samples in terms of their miRNA expression profiles, as well as cluster similarly expressed miRNAs. Supervised cluster analysis grouped EC and LT samples separately for the differentially expressed miRNAs $(\mathrm{p}<0.05)$, before clustering miRNAs based on their expression patterns. Similarly, principal component analysis (PCA) was used to determine how samples and miRNAs cluster, taking gene expression data and transforming it into linearly uncorrelated parameters that could be blotted orthogonally to each other on the score plot in a way that the fewest number of principal components would carry the majority of the variance present in the entire dataset.

Pearson correlation analysis was used to determine how strongly the differentially expressed microRNAs were linearly correlated with one another, where EC and LT groups were analyzed separately and statistically significant correlations had $\mathrm{p}<0.05$. The results of this analysis were graphed as correlation plots, where -1 represented highly negative correlations, 1 represented highly positive correlations, and 0 represented no correlation. 


\subsubsection{Functional characterization of miRNA expression patterns}

MiRNAs were mapped to target mRNA transcripts based on validated and experimentally predicted miRNA:mRNA interactions sourced from online databases including miRecords, mirTarBase, TarBase, DIANA-microT-CDS, ElMMo, TargetScan, MicroCosm, miRDB, PicTar, miRanda, and PITA (Zhang and Storey, 2018). Once potential miRNA:mRNA interactions were identified, the algorithm was used to create regulation scores for each mRNA target based on logarithmically transformed fold-changes and p-values for each miRNA (Zhang and Storey, 2018). Transcript (mRNA) enrichment scores were calculated by summing the product of miRNA score and its affinity for the miRNA for all miRNAs that could possibly target the mRNA transcript. Logistic-regression based gene set enrichment used both the miRNA and mRNA enrichment scores, where significantly enriched gene sets were those with FDR-adjusted $\mathrm{p}<0.05$. Notably, all miRNAs in the gene list were used for the functional characterization of miRNA expression patterns. Enriched gene sets were analyzed against Gene ontology (GO) terms and Kyoto Encyclopedia of Genes and Genomes (KEGG) gene sets to predict which pathways, GO biological processes (GO BPs), and GO molecular functions (GO MFs) may be significantly affected by changes to the miRNA expression profile during LT. A high coefficient (relative to 0) indicates a pathway that is less downregulated by miRNA during LT relative to EC, and a negative coefficient indicates a pathway that may be negatively regulated by miRNAs during LT, relative to the EC.

\subsection{7 cDNA synthesis of miRNA and mRNA for RT-qPCR amplification}

RT-qPCR was used to validate the expression patterns of a representative set of miRNAs. RNA was extracted from samples of EC and LT ground squirrel WAT ( $\mathrm{n}=5$ per condition) as previously described for the small RNA sequencing experiments. Equal amounts $(2 \mu \mathrm{g})$ of RNA were diluted in $7.5 \mu \mathrm{L}$ of autoclaved $\mathrm{ddH}_{2} \mathrm{O}$ for each sample. The polyadenylation of miRNAs sequences was performed using the poly-A polymerase tailing kit (Lucigen, \#PAP5104H). 
Briefly, $1.0 \mu \mathrm{L}$ of $10 \mathrm{X}$ poly-A polymerase buffer, $1.0 \mu \mathrm{L}$ of $1 \mathrm{mM}$ ATP and 0.5 Units of $E$. coli poly-A polymerase were added to each sample, which were incubated in a thermocycler for $37^{\circ} \mathrm{C}$ for 30 mins, and then $95{ }^{\circ} \mathrm{C}$ for 5 mins. The samples were chilled for $5 \mathrm{~min}$ on ice before adding $5 \mu \mathrm{L}$ of $250 \mathrm{pM}$ universal stem-loop adapter primers to each sample. An incubation at $95{ }^{\circ} \mathrm{C}$ for 5 $\min$ and $65^{\circ} \mathrm{C}$ for 5 min was performed to anneal the primers. Reverse transcription was performed as previously described, using a reverse transcription kit (Invitrogen, \#18090010) and $25 \mathrm{mM}$ dNTPs (BioShop, \#NUC001) (Logan and Storey, 2021). The forward primers were miRNA-specific primers that were generated using the human miRNA sequences and the reverse primer for each reaction was the universal primer (Table 5.1). RT-qPCR reactions occurred using the PCR program $95^{\circ} \mathrm{C}(10 \mathrm{sec})$ and $57^{\circ} \mathrm{C}(30 \mathrm{sec})$ for 40 cycles, on a BioRad CFX Connect ${ }^{\mathrm{TM}}$ Real-Time PCR Detection System (\#1855201). A two-fold dilution series using a pooled sample of cDNA from all biological replicates was used to estimate primer efficiency and determine that primer-dimers were not being amplified. Finally, melt curve analysis was used to verify that one product had been amplified in each RT-qPCR experiment.

To analyze the relative levels mRNA transcripts possibly targeted by differentially expressed miRNAs, RNA was prepared as previously described for an $n=4$ biological replicates from independent animals that had been sampled from EC and LT conditions. RNA samples were normalized in a total volume of $10 \mu \mathrm{L}$ DEPC-autoclaved $\mathrm{ddH}_{2} \mathrm{O}$ such that each biological replicate contained $2 \mu \mathrm{g}$ of RNA. Normalized RNA samples were mixed with $1 \mu \mathrm{L}$ of Oligo-dT

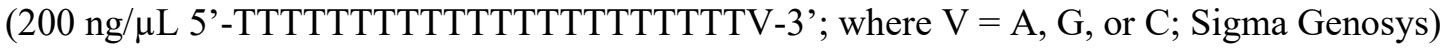
before incubation in a thermocycler at $65^{\circ} \mathrm{C}$ for $5 \mathrm{~min}$. The samples were then chilled on ice for 5 min. Reverse transcription was performed with $4 \mu \mathrm{L}$ of $5 \mathrm{X}$ first-strand buffer (Invitrogen, \#18057018), $2 \mu \mathrm{L}$ of $0.1 \mathrm{M}$ dithiothreitol (DTT) (Invitrogen, \#D1532), $1 \mu \mathrm{L}$ of $10 \mathrm{mM}$ dNTPs (\#NUC001; BioShop, Burlington, Canada), and $1 \mu \mathrm{L}$ of moloney murine leukemia virus (MMLV) reverse transcriptase (Invitrogen, \#28025013). Samples were incubated for $45 \mathrm{~min}$ in 
the thermocycler at $42{ }^{\circ} \mathrm{C}$. RT-qPCR reactions occurred using transcript-specific forward and reverse primers (Table 5.2). RT-qPCR reagents and protocols were performed as previously described (Pellissier et al., 2006). The following PCR program was used on a BioRad CFX Connect ${ }^{\mathrm{TM}}$ Real-Time PCR Detection System: an initial 3 min denaturation phase at $95{ }^{\circ} \mathrm{C}$ followed by 40 cycles of $10 \mathrm{sec}$ at $95{ }^{\circ} \mathrm{C}$ (denaturing), $30 \mathrm{sec}$ at $60^{\circ} \mathrm{C}$ (primer annealing), and 20 sec at $72{ }^{\circ} \mathrm{C}$ (amplification/elongation). To ensure the desired product was amplified, melt-curve analysis was used to determine that only a single peak was present representing a single PCR product. PCR products in SYBR green dye were run on a $1 \%$ agarose gel and visualized to confirm the presence of a single product. Finally, as per minimum information for publication of quantitative (MIQE) guidelines, a two-fold dilution series of the cDNA was also used to determine that the primer pairs were efficient and not amplifying primer-dimers (Bustin et al., 2009).

\subsubsection{Quantification and statistical analysis of RT-qPCR data}

Selected miRNAs whose levels changed according to RNA sequencing $(\mathrm{p}<0.05)$, including 2 miRNAs that increased and 6 miRNAs that decreased during LT were chosen for RTqPCR experiments. Genes possibly targeted by miR-6729 or that were found to fall within differentially regulated KEGG pathways were selected for RT-qPCR validation experiments. Standard curves were prepared using a two-fold dilution series of the pooled WAT samples and miRNAs or mRNA transcripts were amplified using a miRNA-specific forward primer and a universal primer or a pair of gene-specific primers. Only genes that were expressed with $\mathrm{Ct}$ values $<33$ cycles were quantified using the $\Delta \Delta \mathrm{Ct}$ method, as previously described (Taylor et al., 2019). Normfinder was used to assess the stability of four candidate reference genes for miRNA validation experiments including U6 small nuclear RNA, T-methionine tRNA (TMET), and small nucleolar RNAs snord75 and snord96. Then, the ratio of $\Delta \mathrm{Ct}$ values for each gene was calculated using the geometric mean $\triangle \mathrm{Ct}$ of the most stably expressed pair of reference genes, TMET and 
small nucleolar RNA snord75 (Taylor et al., 2019). Similarly, Normfinder was used to assess the stability of G protein subunit beta 2 (GNB), proteasome subunit beta 2 (PSMB), succinate dehydrogenase complex flavoprotein subunit A (SDHA), or peptidylprolyl isomerase A (PPIA), where GNB and PPIA were identified as the best combination of two genes.

RBioplot was used to carry out the statistical analyses and graph the relative expression patterns of RT-qPCR amplified miRNAs (Zhang and Storey, 2016). Before a Student's t-test ( $\mathrm{p}<$ 0.05) was used to determine statistical significance, a Dixon outlier test was performed to identify and remove any outliers present in the data (Efstathiou, 2006). Thus, an $n=5$ per condition was used for EC and LT samples for all genes except an $n=4$ for LT samples was used for miRNAs miR-543, miR-1296-5p, miR-4454, and miR-7704.

\subsubsection{Total protein extraction, Western blotting, and statistical analysis}

Protein extracts were prepared from frozen WAT for each of $n=5$ individual ground squirrels sampled from EC or LT conditions, as previously described (Logan and Storey, 2021). Briefly, several strokes of a glass Dounce homogenizer was used to homogenize $70 \mathrm{mg}$ of frozen WAT 1:2 w:v in ice-cold Cell Signaling Lysis Buffer (EMD Millipore, \#43-040) supplemented with $1 \mathrm{mM}$ sodium orthovanadate, $10 \mathrm{mM}$ sodium fluoride, $10 \mathrm{mM} \beta$-glycerophosphate, 10 $\mu \mathrm{L} / \mathrm{mL}$ Protease Inhibitor cocktail (BioShop, \#PIC001.1), and a few crystals of phenylmethylsulfonyl fluoride. Each sample was kept on ice and was vortexed regularly before centrifugation at $13,500 \times \mathrm{g}$ for $20 \mathrm{~min}$ at $4{ }^{\circ} \mathrm{C}$. The concentration of the supernatant was determined using the Bio-Rad method (Bio-Rad, \#500-0005) and samples were standardized to $10 \mu \mathrm{g} / \mu \mathrm{L}$ with homogenization buffer. A 2 X SDS loading buffer (100 mM Tris-base adjusted to pH 6.8, 4\% w:v SDS, 20\% v:v glycerol, 0.2\% w:v bromophenol blue, 10\% v:v 2mercaptoethanol) added 1:1 v:v to such that the final concentration was $\mu \mathrm{g} / \mu \mathrm{L}$. Equal amounts (25 $\mu \mathrm{g}$ ) of each protein sample were added to $10 \%$ SDS-PAGE gels along with $5 \mu \mathrm{L} 10.5-175$ kDa PiNK Plus pre-stained protein ladder (FroggaBio, \#PM005-0500). 70 min of electrophoresis 
at $180 \mathrm{~V}$ in a Tris-glycine running buffer $(0.25 \mathrm{M}$ Tris-base $\mathrm{pH} 8.0,2.45 \mathrm{M}$ glycine, $0.035 \mathrm{M}$ SDS) was followed by a 90 min transfer of proteins onto PVDF membrane at $160 \mathrm{~mA}$ in transfer buffer (25 mM Tris pH 8.5, $192 \mathrm{mM}$ glycine and 10\% v:v methanol). Blots were blocked for 30 min with $10 \% \mathrm{w}: \mathrm{v}$ powdered skim milk in TBST $(50 \mathrm{mM}$ Tris- $\mathrm{HCl}, 150 \mathrm{mM} \mathrm{NaCl}, 0.05 \% \mathrm{v}: \mathrm{v}$ Tween-20, pH 6.8). Overnight incubation with Cell Signaling primary antibodies for p53 (\#9286), phospho-p53 (S15) (\#9287) and phospho-p53 (S20) (\#2527) diluted 1:1000 v:v in TBST preceded the 30 min application of HRP-linked rabbit IgG secondary antibody (Bioshop, \#APA007P.2) diluted 1:8000 v:v in TBST. Enhanced chemiluminescence (ECL) reagents were used to visualize the protein bands on a Chemi-Genius Bioimaging system (Syngene, Frederick, MD). The PVDF membranes were stained in Coomassie Blue dye (0.25\% w:v Coomassie brilliant blue, $7.5 \% \mathrm{v}: \mathrm{v}$ acetic acid, $50 \%$ methanol) as a protein loading control. ECL and Coomassie Blue stained bands were quantified using GeneTools software. For each lane, chemiluminescent band intensity was standardized against the intensity of a group of Coomassie stained protein bands. 


\subsection{Results}

\subsubsection{Small RNA-sequencing analysis and differential expression of conserved miRNAs}

There were 1,070,540 total reads from four EC samples and three LT samples miRNA libraries that corresponded to microRNAs that perfectly aligned with known human microRNA sequences. One of the LT samples was excluded from RNA-seq preprocessing steps due to having low levels of sequences of the appropriate size. Ground squirrel WAT miRNA sequences perfectly aligned with 635 human miRNAs, but quality control steps were used to remove any miRNAs whose expression levels were lower than 10 counts per million (cpm) in the smallest library, which was control sample 4 with 92,720 read counts. Further, all counts had to be higher than this threshold in 3 or more samples from each condition, control or stress. This yielded 314 conserved miRNAs. There were no miRNAs whose expression changed with respect to condition when an FDR-adjusted p-value $<0.05$ was considered so the analysis herein focused on the 30 miRNAs that were significantly differentially regulated using an unadjusted $\mathrm{p}<0.05$ and a minimum fold-change from EC conditions of 1.5-fold (Table 5.3). Of these, 10 were upregulated during LT and 20 were less expressed (Figure 5.1A).

\subsubsection{Cluster analysis}

Supervised cluster analysis revealed that the miRNA expression profile of EC samples 2 and 3 were more similar to each other than to samples 1 and 4 . Torpor samples 6 and 7 were more similar to each other than to sample 5 (Figure 5.1B). However, the unsupervised hierarchical cluster analysis of centered log transformed (CLR) global miRNA read counts indicated that 
samples do not segregate into perfect EC and LT groups (
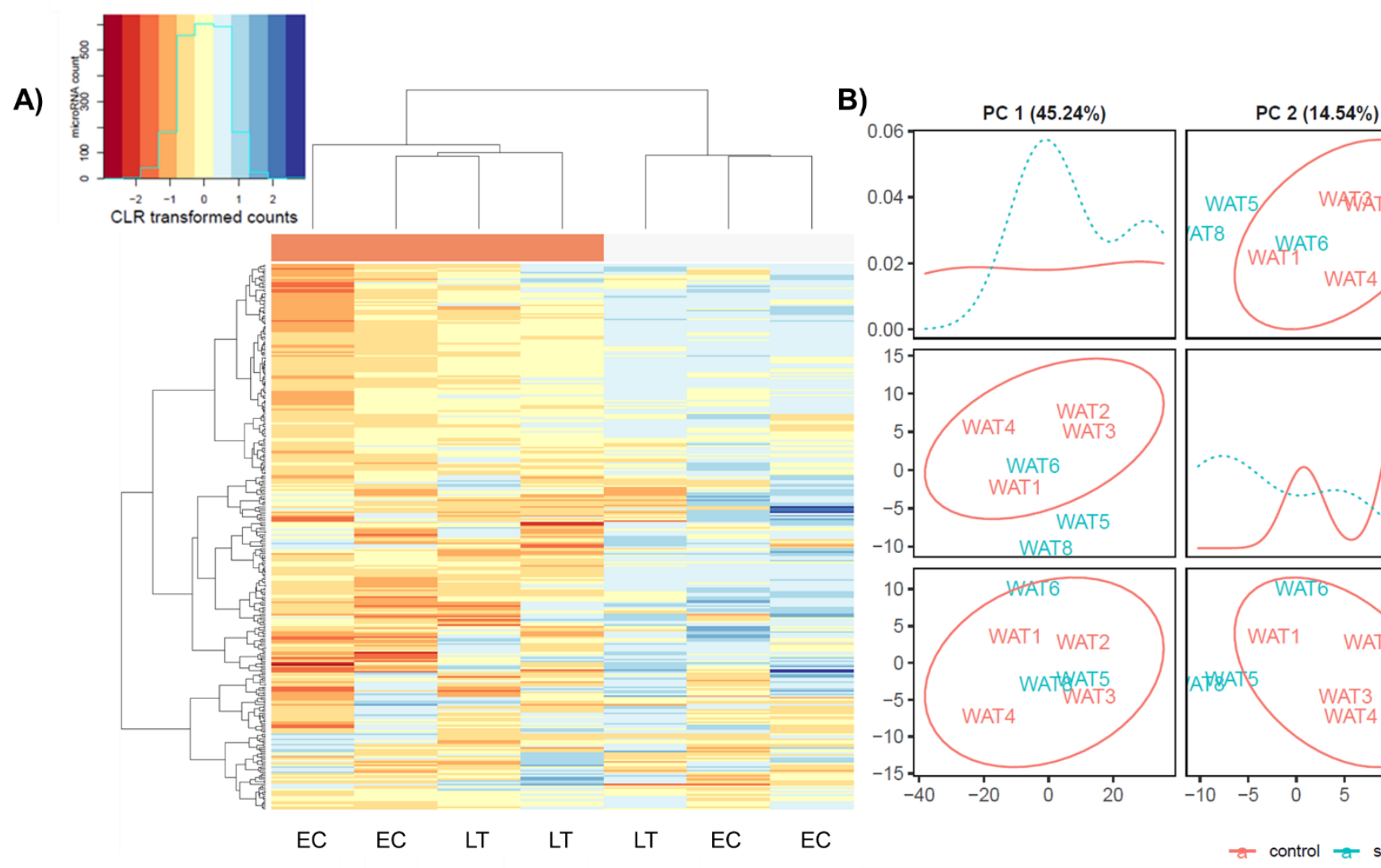

Figure 5.2A). Instead, EC sample 1 and 4 segregated with LT sample 6 and 7 (WAT8 was relabeled as sample 7 due to the removal of WAT7), while control samples 2 and 3 had miRNA expression patterns similar to torpor sample 5. Similarly, the PCA score plots showed that there was overlap between EC and LT samples. The first three components make up $70.89 \%$ 
of the variance in the dataset (
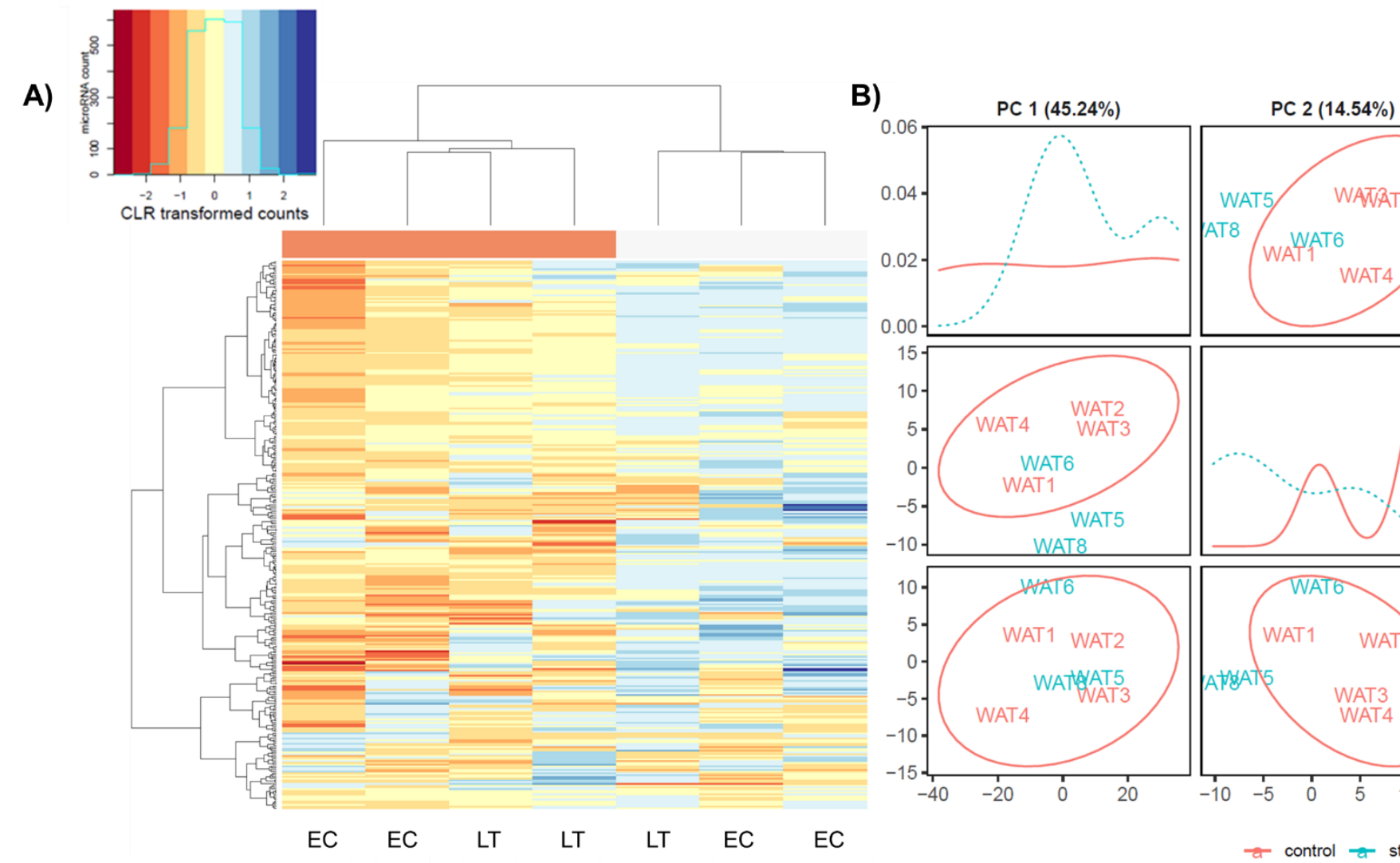

Figure 5.2B).

Pearson correlation heatmaps of miRNA expressed in EC and LT samples from 13-lined ground squirrel WAT show distinct correlations between differentially expressed miRNAs (Figure 5.3, Figure 5.4). There was a total of 30 significant Pearson correlations between miRNAs in control samples, where 22 pairs of miRNAs were positively linearly correlated and 8 were negatively correlated. Similarly, for LT samples, there were 36 pairs of miRNAs that were linearly correlated and only four were negatively correlated. There was a cluster of positively correlated miRNAs in the LT samples involving many of the miRNAs that appeared to cluster in the supervised cluster analysis heatmap. For example, significant Pearson correlations were noted between miR-194 and miR-26b-3p, miR-3120-3p, miR-431-5p, miR-543, miR-6500-5p, miR6529-3p, and miR-6869-5p (Figure 5.3B). 


\subsubsection{Gene set analysis}

To determine how global changes in the levels of miRNAs level may influence cellular processes, experimentally validated and predicted miRNA:mRNA interactions for each of the 314 miRNAs that were identified in ground squirrel WAT samples, as previously described (Zhang and Storey, 2018). Enrichment scores for miRNAs and mRNAs were used to identify KEGG pathways (Figure 5.5, Table 5.4), GO biological processes (GO BPs) (Supplemental Figure 5.1, Table 5.5), and GO molecular functions (GO MFs) (Supplemental Figure 5.2, Table 5.6) that were significantly enriched (FDR-adjusted $p$-values $<0.05$ ). The histograms show GO BPs and GO MFs that were enriched based on FDR-adjusted $\mathrm{p}<0.0001$ or $<0.01$ respectively.

Of the 45 KEGG Pathways that were significantly enriched, only 5 were positively regulated, meaning less inhibited by miRNAs during LT. These pathways included nucleotide and base excision repair, olfactory transduction, drug metabolism cytochrome P450 and neuroactive ligand receptor interaction. Pathview analysis shows genes that were postulated to be regulated by miRNAs during $\mathrm{LT}$, based on global miRNA expression patterns for several pathways of interest (Supplemental Figure 5.3-5.5). Similarly, among the 19 GO BPs that were significantly less inhibited based on miRNA expression patterns, during LT, many pertained to detection and response to stimuli (7 GO BPs), DNA damage and repair (4 GO BPs) and lipid metabolism (3 GO BPs). Out of 144 GO MFs, 127 were negatively enriched and included a variety of functions including activin binding, insulin receptor substrate binding, transcription coactivator/repressor and cofactor binding, miRNA and pre-mRNA binding (Supplemental

\section{Figure 5.2).}

Some of the 40 KEGG pathways that were downregulated by miRNAs during LT (Figure 5.5, Table 5.4) included cancer-specific signaling pathways, as well as signaling pathways centered around p53, cell cycle, NOD-like receptors, toll-like receptors (TLRs), transforming growth factor (TGF)-beta, insulin, mechanistic target of rapamycin (mTOR), 
mitogen-activated protein kinase (MAPK), adipocytokines, and wingless and int-1 (WNT), to name a few. Consistently, many of the 1033 GO BPs that were significantly enriched included gene expression, response to stress, cell cycle/differentiation/death, G-coupled protein receptor signaling, protein auto-processing, and positive regulation of protein import into nucleus translocation, among others (Supplemental Figure 5.1). Of the 17 GO MFs that were predicted to be less inhibited by miRNAs during torpor, based on the overall miRNA expression profile, many had functions in signaling receptor binding activity or ion channel activity (Supplemental Figure 5.2). Pathway analysis showed that the majority of genes involved in p53 signaling and NOD-like receptor signaling pathway are likely inhibited by miRNAs during LT (Supplemental Figure 5.4, Supplemental Figure 5.5).

\subsubsection{RT-qPCR and Western blotting experiments}

RT-qPCR experiments were performed to validate the expression patterns of a subset of miRNAs as well as the levels of some mRNAs predicted to be targets of differentially expressed miRNAs (Figure 5.6). Most RT-qPCR amplified miRNAs did not change during LT relative to EC. The exception to this was miR-6729-5p, a miRNA that increased by approximately 1.8 -fold.

RT-qPCR was also used to further investigate the expression patterns of genes that could be regulated by miRNAs during LT, such as those pertaining to the KEGG pathways for nucleotide and base excision repair. Transcript levels for DNA ligases 1 and 4 (LIG1 and LIG4, respectively), proliferating cell nuclear antigen (PCNA), single-strand-selective monofunctional uracil-DNA glycosylase 1 (SMUG1), and nei-like DNA glycosylase 3 (NEIL3) did not differ from the control but LIG3 levels were reduced by nearly 50\%. Genes within downregulated KEGG pathways focused on p53 signaling and the cell cycle were also assessed, including checkpoint kinase 1 (CHK1), p53, p21, minichromosome maintenance complex component 5 (MCM5), and mouse double minute 2 homolog (MDM2). There was only a decrease in the relative transcript levels of MDM2 to nearly half of the EC level. 
Western blotting was performed to further characterize how p53 signaling was regulated during LT (Figure 5.7). There were no changes in $\mathrm{p} 53$ total protein levels but there were significant increases in p-p53 S15 and p-p53 S20 levels to approximately 1.6-fold and 1.8-fold the control value, respectively. 


\subsection{Discussion}

MicroRNA expression patterns were explored in the WAT of hibernating 13-lined ground squirrels to determine how these extreme animals with chronic obesity may use miRNAs to regulate metabolism and tissue homeostasis. Small RNA sequencing revealed changes in the levels of 30 miRNAs, considering miRNAs whose expression changed more than 1.5 -fold ( $\mathrm{p}=$ 0.05). Twenty of these miRNAs were downregulated during torpor and ten were upregulated compared to the control group.

These results mirrored the trends seen in a small-scale study exploring the role of twenty miRNAs implicated in adipogenesis and obesity in a model of natural obesity, the 13-lined ground squirrel (Wu et al., 2014). The authors found three miRNAs were upregulated and six miRNAs were downregulated. Eight of those nine highly conserved miRNAs identified by RTqPCR were also identified in the current study. However, there was almost no overlap in the expression patterns of miRNAs in the small-scale study and the current one, perhaps due to differences in normalization practices. Though the reference genes chosen for the RT-qPCR experiments met the necessary qualifications to be considered suitable controls, perhaps there would have been more similarities between the RNA-sequencing dataset, the RT-qPCR data from the current study, and those from the previous study if the reference gene used was the same. In a semi-quantitative RT-PCR study, miRNA levels across multiple samples are normalized to a single reference gene or the geometric mean of several reference genes that are believed to be unaffected by the condition parameter. Similarly, when analyzing miRNA-sequencing data, the level of each miRNA must be normalized to read depth for each sample. Thus, performing a $\log \mathrm{CPM}$ conversion corrects for differences in sequencing depth by dividing read counts by library size for each sample. Then, center scaling (quantile normalization) is used to normalize by gene distribution, which controls for differences in sequencing across samples. While it is possible to identify suitable reference genes for RT-qPCR studies from the results of an RNA 
sequencing study, it is not common practice to use a housekeeping gene or a set of housekeeping genes for normalization of RNA-sequencing data.

Another study done on pooled WAT samples from hibernating bats also showed that few conserved miRNAs are differentially regulated between control and torpor conditions (Yuan et al., 2015). Mir-200c-3p, which decreased over three-fold in the current study and miR-200b-3p, which increased over four-fold in the study on bats, are identical in sequence except that miR$200 \mathrm{~b}-3 \mathrm{p}$ is one adenosine longer. Apart from this miRNA, there was no overlap in miRNAs that were differentially regulated in both hibernating ground squirrel and bat WAT, which suggests species-specific use of miRNAs to regulate metabolism during torpor.

Both the small-scale study and the current study on 13-lined ground squirrel WAT found a decrease in miR-31. MiRNA-31 was shown to be upregulated by tumor necrosis factor $(\mathrm{TNF} \alpha) /$ nuclear factor kappa $\mathrm{B}(\mathrm{NF} \kappa \mathrm{B})$ and IL-6/signal transducer and activator of transcription 3 (STAT3) signaling in inflamed keratinocytes repairing from injury, and miR-31 upregulation correlated with increased Ras/MAPK signaling given that it can regulate inhibitors of this signaling pathway (Shi et al., 2018). In this study, miR-31 was shown to only regulate cell differentiation, proliferation, and death in wounded tissue and not healthy tissue. Thus, a decrease in miR-31-5p could suggest that there is little to no physical damage in WAT during LT or ground squirrels actively downregulate this miRNA to prevent the degradation of inhibitors of energy expensive cell division and development pathways. Consistent with the notion that WAT is not injured during torpor, the decrease in miR-31-5p levels correlated with significantly less extracellular signal-regulated kinase (ERK1/2) phosphorylation in ground squirrel WAT during LT (Rouble et al., 2014).

The miRNA profile also suggested that inflammatory pathways may be inhibited by miRNAs. The KEGG Pathway NOD-like receptor signaling was more targeted by miRNAs during torpor. This pathway activates the innate immune response in the presence of pathogenic 
molecular patterns (PAMPs) from viral or bacterial infection and damage-associated molecular patterns (DAMPs) from cell stress. The latter of these is more likely to occur during hibernation since most parasites are inhibited in their hibernator hosts at low body temperatures (Cahill et al., 1967). Pathview analysis suggested that NOD-like receptors such as NOD-containing protein 2 (NOD2), NOD-, LRR- and pyrin domain-containing protein 3 (NLRP3), NLRP6, absent in melanoma 2 (AIM2), and apoptosis-associated speck-like protein containing a caspase activation and recruitment domain (CARD) (ASC) were unlikely targets of miRNAs during torpor. Instead, upstream regulators of NOD-like signaling and genes downstream NOD-like receptor activation were identified as miRNA-targeted genes during torpor. Notable upstream regulators included thioredoxin interacting protein (TXNIP), mitochondrial antiviral signaling protein (MAVS), and heat shock protein 90 (HSP90) and some downstream genes highlighted as targets of miRNA regulation included caspase-1, gasdermin D, IL-1 $\beta$, TGF $\beta$-activated kinase 1 (TAK1), TAK1 binding protein (TAB), interferon regulatory factor (IRF) $3 / 7$ and IкB kinase (IKK) $\alpha / \beta$. Consistent with this, the relative levels of inflammasome receptor genes (e.g. NLRP3, AIM2, ASC) were stable throughout the torpor-arousal cycle in 13-lined ground squirrel WAT, but caspase-1 activity was significantly decreased during torpor and arousal, relative to EC (Logan and Storey, 2021). Together, these results demonstrate a role for miRNAs in suppressing the activation of the inflammasome in WAT which would inhibit inflammation and pyroptosis.

In addition to miRNAs potentially controlling wound healing and inflammation, the results of KEGG Pathway analysis seemed to suggest that base excision repair (BER) and nucleotide excision repair (NER) pathways were generally less inhibited by miRNAs during LT (Figure 5.5). This is important because DNA repair processes may have important roles in maintaining tissue viability amid the cellular perturbations and extreme metabolic changes of the hibernation season. DNA repair mechanisms used by torpid animals have yet to be fully characterized. Specifically, BER genes coloured red in Pathview analysis were predicted to be 
less inhibited by miRNAs during LT and genes shown in blue were postulated to be targeted by miRNAs during LT (Supplemental Figure 5.3). Several of these genes including LIG1, LIG3, LIG4, NEIL3, SMUG1, and PCNA were measured using RT-qPCR to determine if they may indeed be less inhibited by miRNAs during torpor. The levels of almost all of these mRNAs were maintained at EC levels during LT, suggesting they may not be primer targets for miRNA regulation, as expected. An exception was LIG3, which was downregulated in LT ground squirrel WAT to approximately $50 \%$ of the control level. LIG1, LIG3 and LIG4 serve different and overlapping functions in terms of DNA repair. LIG1 and LIG4 are exclusively present in the nucleus, with LIG1 having important roles in DNA replication since it facilitates the joining of Okazaki fragments on the lagging strand (Sallmyr et al., 2020). LIG3, like LIG1, contributes to single stranded DNA break repair, as well as BER and NER, but LIG1 is the predominant ligase when the cell is dividing. LIG4 is involved in non-homologous end joining (NHEJ) of double stranded DNA breaks throughout the cell cycle (Sallmyr et al., 2020). LIG3 is interesting because it can repair and replicate nuclear DNA when LIG1 levels are low, but it is also present in the mitochondria where it facilitates mitochondrial DNA replication and repair (Sallmyr et al., 2020). Therefore, LIG3 may be downregulated to limit mitochondrial replication and the consumption of metabolic fuel during LT, whereas LIG1, LIG4, and other genes implicated in BER/NER may be maintained at EC levels to prevent and repair nuclear DNA damage.

One of the miRNAs that was significantly upregulated according to miRNA-sequencing and RT-qPCR was miR-6729-5p. Other RT-qPCR validated miRNAs included upregulated miRNAs (miR-21-3p in addition to miR-6729-5p) and downregulated miRNAs (miR-200c-3p, miR-205-5p, miR-543, miR-1296-5p, miR-4454, and miR-7704). Differences in miRNAsequencing and RT-qPCR results may stem from differences in reference points, as previously discussed, or from differences in WAT sampling. For instance, heavily vascularized regions of WAT may express more miRNAs involved in regulating angiogenesis and endothelial cell 
proliferation and differentiation. Indeed, angiogenesis and vasculature development were identified as GO BPs that were more likely to be inhibited during LT based on the global expression patterns of miRNAs identified via sequencing (Supplemental Figure 5.1). Further, cold-acclimated and hibernating hamsters have been shown to produce patches of multilocular beige-like cells and expressed higher levels of UCP1 compared to non-hibernating hamsters (Chayama et al., 2019). This suggests that differences in the proportions of white and beige adipocytes sampled for the small-scale study, miRNA-sequencing, and RT-qPCR validation experiments could also explain the differences in relative miRNA levels. Finally, it should be emphasized that WAT was sampled from wild-captured ground squirrels. This is important because ground squirrels may differ in their age and life-history. This aspect of study design allows for the detection of changes in gene expression that are likely to be representative of wild populations. However, future studies could use more biological replicates and sample from laboratory animals where age and life history are known to examine gene expression with more statistical power.

In terms of the importance of the upregulation of miR-6729-5p, miRNA-mRNA interaction experiments suggest this miRNA can inhibit genes involved in cell cycle and DNA replication, including origin recognition complex subunit 1 (ORC1), ORC6, MCM5, MDM2, cyclin B1 (CCNB1/CycB), calmodulin 3 (CALM3), and DNA replication helicase/nuclease 2 (DNA2). MiRNA-mediated downregulation of the cell cycle would be consistent with reports of suppressed cell cycle and cell proliferation during torpor in various tissues from ground squirrel (Wu and Storey, 2012). Furthermore, the functions of several mRNA targets of miR-6729-5p could be classified into some of the most significantly downregulated GO biological processes, such as negative regulation of cell cycle, negative regulation of cell death, and negative regulation of protein metabolic processes. To investigate if miRNAs could downregulate these processes in 
WAT, the relative expression of five genes known to play important roles in cell cycle and p53 regulation was measured using RT-qPCR (Figure 5.6B).

MDM2, which was downregulated during LT, typically plays important roles in tightly controlling the expression of p53, a gene known to regulate signaling pathways involved in hypoxia, endoplasmic reticulum stress, DNA damage, apoptosis, and cell cycle arrest (Hernández-Monge et al., 2016). P53 responds to cell stress, including temperature changes and DNA damage, by inhibiting the cell cycle, upregulating genes involved in DNA repair, and inciting cell death if needed (Hefler et al., 2015). MDM2 can polyubiquitinate the $\mathrm{N}$-terminus of p53, resulting in its degradation, in order to keep p53 levels low (Hernández-Monge et al., 2016). Therefore, MDM2 downregulation by miRNAs such as miR-6729-5p could enable $\mathrm{p} 53$-mediated cellular senescence by reducing p53 degradation. Using western blotting, it was determined that p53 protein levels were maintained, while p53 phosphorylation was increased at both serine 15 and serine 20 during LT in ground squirrel WAT (Figure 5.7). Phosphorylation at S15 and S20 are mediated by DNA protein kinase and $\mathrm{CHK} 1 / \mathrm{CHK} 2$, respectively, in response to cell stress such as DNA damage. These post-translational modifications are associated with increased p53 stability. First and foremost, increased p-p53 (S15 and S20) supports the notion that p53 may be stabilized during LT, which is consistent with miRNA-mediated downregulation of MDM2 and maintained p53 protein levels. Additionally, increased p53 phosphorylation at S15 and S20 could suggest that WAT may experience DNA damage during torpor. As previously discussed, DNA repair KEGG pathways were predicted to be less inhibited by miRNAs, and RT-qPCR analysis of some DNA replication and repair genes supports the notion that this pathway is uninhibited. Other hibernating ground squirrel tissues also seem to upregulate p53 signaling. In ground squirrel skeletal muscle, p53 was elevated at the transcript and protein levels in 13-lined ground squirrel skeletal muscle, its nuclear protein levels increased, and so did its DNA binding activity (Hefler et al., 2015). Similarly, measurement of relative gene expression of p53, its upstream 
regulators, and downstream genes revealed that p53 signaling may be important in goldenmantled ground squirrel liver during torpor and interbout arousal, compared to summer active ground squirrels (Pan et al., 2014). Together, these results suggest many ground squirrel tissues activate p53 signaling in response to cell stress, and this transcription factor could play an important role in downregulating energy expensive processes like the cell cycle, while upregulating DNA repair pathway genes.

\subsection{Conclusion}

In summary, small RNA sequencing of EC and LT ground squirrel white adipose tissue revealed a possible role for miRNAs in the regulation of metabolism during torpor. For instance, the decrease in miR-31 during torpor suggested WAT is not likely to be damaged during LT, if this miRNA only regulates wound repair mechanisms in damaged tissue. Similarly, it was predicted that some KEGG pathways like NOD-like receptor signaling would be less inhibited during torpor, but genes within this pathway that would be more inhibited by miRNAs included upstream regulators of NOD-like receptors, as well as NOD-like receptor activated genes. This

finding was consistent with a recent study on canonical inflammasome signaling in the white and brown adipose depots of ground squirrels. DNA repair pathways were also predicted to be less inhibited during LT, which was confirmed by RT-qPCR. None of the assessed DNA replication and repair genes were differentially expressed with the exception of LIG3, which was downregulated, possibly as part of a mechanism to inhibit mitochondrial replication in LT. Finally, miR-6729-5p was one of the significantly upregulated genes whose expression was confirmed using RT-qPCR. Interaction analysis revealed that many of its target genes could play roles in negatively enriched KEGG Pathways such as p53 signaling and the cell cycle. However, only a MDM2, a negative regulator of p53 signaling was downregulated during LT. Western blotting demonstrated ground squirrel maintained p53 protein levels during LT and may activate the protein using post-translational modifications, instead. Thus, post-transcriptional regulation 
may be used by the WAT of fat-but-fit hibernators to limit torpor-induced inflammation, protect against and repair DNA damage, and regulate energetically expensive processes such as p53 signaling and the cell cycle. 


\subsection{References}

Biggar, K.K., Storey, K.B., 2014. Insight into temperature-dependent microRNA function in mammalian hibernators. Temperature 1, 84-86. https://doi.org/10.4161/temp.29656

Boyer, B.B., Barnes, B.M., 1999. Molecular and metabolic aspects of mammalian hibernation. Bioscience 49, 713-724. https://doi.org/10.2307/1313595

Burnett, W.L., 1914. The striped spermophiles or ground squirrels of Colorado (Citellus tridecemlineatus pallidus and Citellus tridecemlineatus parvus).

Bustin, S.A., Benes, V., Garson, J.A.J., Hellemans, J., Huggett, J., Kubista, M., Mueller, R., Nolan, T., Pfaffl, M.W., Shipley, GL Vandesompele, J., Wittwer, C.T., Shipley, G.L., Vandesompele, J., Wittwer, C.T., 2009. The MIQE guidelines: minimum information for publication of quantitative real-time PCR experiments. Clin. Chem. 55, 611-622. https://doi.org/10.1373/clinchem.2008.112797

Cahill, J.E., Lewart, R.M., Jaroslow, B.N., 1967. Effect of hibernation on course of infection and immune response in Citellus tridecemlineatus infected with Nippostrongylus brasiliensis. J. Parasitol. 53, $110-115$.

Chayama, Y., Ando, L., Sato, Y., Shigenobu, S., Anegawa, D., Fujimoto, T., Taii, H., Tamura, Y., Miura, M., Yamaguchi, Y., 2019. Molecular basis of white adipose tissue remodeling that precedes and coincides with hibernation in the Syrian hamster, a food-storing hibernator. Front. Physiol. 10, 1-16. https://doi.org/10.3389/fphys.2018.01973

Efstathiou, C.E., 2006. Estimation of type I error probability from experimental Dixon's “Q” parameter on testing for outliers within small size data sets. Talanta 69, 1068-1071. https://doi.org/10.1016/j.talanta.2005.12.031

Geiser, F., 2004. Metabolic rate and body temperature reduction during hibernation and daily torpor. Annu. Rev. Physiol. 66, 239-74. https://doi.org/10.1146/annurev.physiol.66.032102.115105

Hales, C.M., Carroll, M.D., Fryar, C.D., Ogden, C.L., 2020. Prevalence of Obesity and Severe Obesity Among Adults: United States, 2017-2018. NCHS Data Brief 1-8.

Hefler, J., Wu, C., Storey, K.B., 2015. Transcriptional activation of p53 during cold induced torpor in the 13-lined ground squirrel Ictidomys tridecemlineatus. Biochem. Res. Int. 2015, 1-11. https://doi.org/10.1155/2015/731595

Hernández-Monge, J., Rousset-Roman, A.B., Medina-Medina, I., Olivares-Illana, V., 2016. Dual function of MDM2 and MDMX toward the tumor suppressors p53 and RB. Genes Cancer 7, 278-287. https://doi.org/10.18632/genesandcancer.120

Kalvari, I., Nawrocki, E.P., Argasinska, J., Quinones-Olvera, N., Finn, R.D., Bateman, A., Petrov, A.I., 2018. Non-coding RNA analysis using the Rfam database. Curr. Protoc. Bioinforma. 62, e51. https://doi.org/10.1002/cpbi.51

Kayser, B., Verges, S., 2013. Hypoxia, energy balance and obesity: from pathophysiological mechanisms to new treatment strategies. Obes. Rev. 14, 579-592. https://doi.org/10.1111/obr.12034

Kisser, B., Goodwin, H.T., 2012. Hibernation and overwinter body temperatures in free-ranging 13-lined ground squirrels, Ictidomys tridecemlineatus. Am. Midl. Nat. 167, 396-409. https://doi.org/10.1674/0003-0031-167.2.396

Kozomara, A., Birgaoanu, M., Griffiths-Jones, S., 2019. MiRBase: From microRNA sequences to function. Nucleic Acids Res. 47, D155-D162. https://doi.org/10.1093/nar/gky1141

Law, C.W., Chen, Y., Shi, W., Smyth, G.K., 2014. voom: precision weights unlock linear model analysis tools for RNA-seq read counts. Genome Biol. 15, R29. https://doi.org/10.1186/gb-2014-15-2-r29

Li, H., Handsaker, B., Wysoker, A., Fennell, T., Ruan, J., Homer, N., Marth, G., Abecasis, G., Durbin, R., 
2009. The sequence alignment/map format and SAMtools. Bioinformatics 25, 2078-2079. https://doi.org/10.1093/bioinformatics/btp352

Logan, S.M., Luu, B.E., Storey, K.B., 2016. Turn down genes for WAT? Activation of anti-apoptosis pathways protects white adipose tissue in metabolically depressed 13-lined ground squirrels. Mol. Cell. Biochem. 416, 47-62. https://doi.org/10.1007/s11010-016-2695-0

Logan, S.M., Storey, K.B., 2021. Inflammasome signaling could be used to sense and respond to endogenous damage in brown but not white adipose tissue of a hibernating ground squirrel. Dev. Comp. Immunol. 114, 103819. https://doi.org/10.1016/j.dci.2020.103819

McMullen, D.C., Hallenbeck, J.M., 2010. Regulation of Akt during torpor in the hibernating ground squirrel, Ictidomys tridecemlineatus. J. Comp. Physiol. B Biochem. Syst. Environ. Physiol. 180, $927-$ 934. https://doi.org/10.1007/s00360-010-0468-8

Pan, P., Treat, M.D., van Breukelen, F., 2014. A systems-level approach to understanding transcriptional regulation by p53 during mammalian hibernation. J. Exp. Biol. 217, 2489-2498. https://doi.org/10.1242/jeb.103614

Pellissier, F., Glogowskib, C.M., Heinemannb, S.F., Balliveta, M., Ossipowa, V., 2006. Lab assembly of a low-cost, robust SYBR green buffer system for quantitative real-time polymerase chain reaction. Anal. Biochem. 350, 310-312. https://doi.org/10.1016/j.ab.2005.12.002

Rouble, A.N., Tessier, S.N., Storey, K.B., 2014. Characterization of adipocyte stress response pathways during hibernation in 13-lined ground squirrels. Mol. Cell. Biochem. 393, 271-282. https://doi.org/10.1007/s11010-014-2070-y

Sai lakshmi, S., Agrawal, S., 2008. piRNABank: A web resource on classified and clustered Piwiinteracting RNAs. Nucleic Acids Res. 36. https://doi.org/10.1093/nar/gkm696

Sallmyr, A., Rashid, I., Bhandari, S.K., Naila, T., Tomkinson, A.E., 2020. Human DNA ligases in replication and repair. DNA Repair (Amst). 93, 102908. https://doi.org/10.1016/j.dnarep.2020.102908

Sheriff, M.J., Fridinger, R.W., Tøien, Ø., Barnes, B.M., Buck, C.L., 2013. Metabolic rate and prehibernation fattening in free-living arctic ground squirrels. Physiol. Biochem. Zool. 86, 515-27. https://doi.org/10.1086/673092

Shi, J., Ma, X., Su, Y., Song, Y., Tian, Y., Yuan, S., Zhang, X., Yang, D., Zhang, H., Shuai, J., Cui, W., Ren, F., Plikus, M. V., Chen, Y., Luo, J., Yu, Z., 2018. MiR-31 mediates inflammatory signaling to promote re-epithelialization during skin wound healing. J. Invest. Dermatol. 138, 2253-2263. https://doi.org/10.1016/j.jid.2018.03.1521

Statistics Canada, 2019. Overweight and obese adults, 2018 [WWW Document]. Heal. Fact Sheets. URL https://www150.statcan.gc.ca/n1/pub/82-625-x/2019001/article/00005-eng.htm

Storey, K.B., 2010. Out cold: biochemical regulation of mammalian hibernation - A mini-review. Gerontology 56, 220-230. https://doi.org/10.1159/000228829

Taylor, S.C., Nadeau, K., Abbasi, M., Lachance, C., Nguyen, M., Fenrich, J., 2019. The ultimate qPCR experiment: producing publication quality, reproducible data the first time. Trends Biotechnol. 37, 761-774. https://doi.org/10.1016/j.tibtech.2018.12.002

Wang, L.C.H., Lee, T.F., 1996. Torpor and hibernation in mammals: metabolic, physiological, and biochemical adaptations, in: Pappenheimer, J.R., Fregly, M.J., Blatties, C.M. (Eds.), Handbook of Physiology - Environmental Physiology. Oxford University Press, Oxford, UK, pp. 507-532. https://doi.org/10.1002/cphy.cp040122

Ward, J.H., 1963. Hierarchical grouping to optimize an objective function. J. Am. Stat. Assoc. 58, 236. https://doi.org/10.2307/2282967 
Wu, C.-W., Biggar, K.K., Storey, K.B., 2014. Expression profiling and structural characterization of microRNAs in adipose tissues of hibernating ground squirrels. Genomics. Proteomics Bioinformatics 12, 284-291. https://doi.org/10.1016/j.gpb.2014.08.003

Wu, C.-W., Storey, K.B., 2012. Pattern of cellular quiescence over the hibernation cycle in liver of 13-lined ground squirrels. Cell Cycle 11, 1714-1726. https://doi.org/10.4161/cc.19799

Yuan, L., Geiser, F., Lin, B., Sun, H., Chen, J., Zhang, S., 2015. Down but not out: the role of microRNAs in hibernating bats. PLoS One 10, e0135064. https://doi.org/10.1371/journal.pone.0135064

Zhang, J., Hadj-Moussa, H., Storey, K.B., 2020. Marine periwinkle stress-responsive microRNAs: A potential factor to reflect anoxia and freezing survival adaptations. Genomics 112, 4385-4398. https://doi.org/10.1016/j.ygeno.2020.07.036

Zhang, J., Hadj-Moussa, H., Storey, K.B., 2016. Current progress of high-throughput microRNA differential expression analysis and random forest gene selection for model and non-model systems: an R implementation. J. Integr. Bioinform. 13, 306. https://doi.org/10.2390/biecoll-jib-2016-306

Zhang, J., Storey, K.B., 2018. RBiomirGS: An all-in-one miRNA gene set analysis solution featuring target mRNA mapping and expression profile integration. PeerJ 2018, 1-17. https://doi.org/10.7717/peerj.4262

Zhang, J., Storey, K.B., 2016. RBioplot: an easy-to-use R pipeline for automated statistical analysis and data visualization in molecular biology and biochemistry. PeerJ 4, e2436.

https://doi.org/10.7717/peerj.2436 


\subsection{Tables}

Table 5.1: Primers used in RT-qPCR analysis of miRNAs

\begin{tabular}{ll}
\hline Gene & $\mathbf{5}^{\prime}-\mathbf{3}$ ' primer \\
\hline TMET & ACACTCCAGCTGGGAGCAGAGTGGCGCAG \\
\hline snord75 & ACACTCCAGCTGGGTTATGGATTCGCACG \\
\hline miR-21-3p & ACACTCCAGCTGGGTAGCTTATCAGACTG \\
\hline miR-200c-3p & ACACTCCAGCTGGGTAATACTGCCGGGTAAT \\
\hline miR-205-5p & ACACTCCAGCTGGGTCCTTCATTCCACCG \\
\hline miR-543 & ACACTCCAGCTGGGAAACATTCGCGGTGC \\
\hline miR-1296-5p & ACACTCCAGCTGGGTTAGGGCCCTGGCTC \\
\hline miR-4454 & ACACTCCAGCTGGGGGATCCGAGTCACGG \\
\hline miR-6729-5p & ACACTCCAGCTGGGTGGGCGAGGGCGGCT \\
\hline miR-7704 & ACACTCCAGCTGGGCGGGGTCGGCGGCGA \\
\hline Universal Primer (reverse) & ACCGGTTCTCGTCCGATCACCGAAGT \\
\hline
\end{tabular}


Table 5.2: Primers used in RT-qPCR analysis of mRNAs

\begin{tabular}{lll}
\hline Gene & Forward primer (5'-3') & Reverse Primer (5'-3') \\
\hline GNB & GCCTTGGGGTCACTGATGAT & GGGTTGGGACCGTTAGTTCC \\
\hline PSMB & AGGGGCCAGCACTCTACTAC & GAGAGTCAGGAAGGCACCAT \\
\hline SDHA & CTGTGAAGGGCTCTGACTGG & TTCTCTAGCTCGACCACGGA \\
\hline PPIA & GCAAGTCCATCTACGGGGAG & CTCAGTCTTGGCAGTGCAGA \\
\hline LIG1 & ACTTGGGACTGTAGGGTACG & TACTTCGCTGCATGATGGTCT \\
\hline LIG3 & TCCCCAACAGCAAAGGCAAT & CCCAGTGAAGATGTCCAGCA \\
\hline LIG4 & TGTGGGAGGCTATTGGGGTA & GGCTTCTCTGCTACTGCACA \\
\hline PCNA & CGGCATTAAACGGTTGCAGA & GGAGTTTAGGCTTCCCGAGG \\
\hline SMUG1 & TTTGGAGTGGGGGATGGTTG & CCCAAGCTCACCTCAGTTCT \\
\hline NEIL3 & AAAACAGACAGGTCGGATGCT & TGATGATGTTGCCTACCCCG \\
\hline CHK1 & ACGGTCACAGGAGAGAAGGA & CACCCCTGCCATGAGTTGAT \\
\hline p53 & TGGTGTTTTGGGGAGAGGTG & CGGATGGCTGGTTGTAGGTT \\
\hline p21 & TCTGTGATTCCGCCCCTTTC & ATGATGACCCCACTTGGCTG \\
\hline MCM5 & AAGGCTGGCATCACTACCAC & AGATCATGTCGAAGCGGGAC \\
\hline MDM2 & AGAAGAGGGCTTGGATGTGC & GGGAGGCTTGCGTGATCTTA \\
\hline
\end{tabular}


Table 5.3: Differential expression of miRNAs using a p-value of 0.05 as the threshold for significance. Log2-transformed fold-changes in gene expression and false discovery rate-adjusted p-values are also shown for the differentially expressed miRNAs.

\begin{tabular}{|l|l|l|l|}
\hline Gene & logFC & P Value & FDR-adjusted P Value \\
\hline hsa-miR-130296-5p & -1.200 & 0.038 & 0.456 \\
\hline hsa-miR-140-5p & 1.473 & 0.048 & 0.460 \\
\hline hsa-miR-145-5p & -0.732 & 0.048 & 0.460 \\
\hline hsa-miR-181c-3p & 2.092 & 0.015 & 0.368 \\
\hline hsa-miR-193b-5p & -0.628 & 0.044 & 0.460 \\
\hline hsa-miR-194-5p & -2.353 & 0.011 & 0.368 \\
\hline hsa-miR-195-5p & 0.697 & 0.017 & 0.368 \\
\hline hsa-miR-200c-3p & -3.014 & 0.021 & 0.368 \\
\hline hsa-miR-205-5p & -1.929 & 0.014 & 0.368 \\
\hline hsa-miR-206 & -1.928 & 0.021 & 0.368 \\
\hline hsa-miR-21-3p & 2.564 & 0.001 & 0.271 \\
\hline hsa-miR-221-3p & 0.985 & 0.012 & 0.368 \\
\hline hsa-miR-26b-3p & -2.235 & 0.003 & 0.286 \\
\hline hsa-miR-3074-5p & -0.588 & 0.033 & 0.444 \\
\hline hsa-miR-30c-5p & -1.298 & 0.004 & 0.286 \\
\hline hsa-miR-3120-3p & -1.987 & 0.049 & 0.460 \\
\hline hsa-miR-31-5p & -2.618 & 0.006 & 0.331 \\
\hline hsa-miR-338-5p & 1.746 & 0.030 & 0.444 \\
\hline hsa-miR-3609 & 1.689 & 0.016 & 0.368 \\
\hline hsa-miR-365a-5p & -1.991 & 0.040 & 0.460 \\
\hline hsa-miR-431-5p & -1.779 & 0.036 & 0.449 \\
\hline hsa-miR-4454 & -1.014 & 0.030 & 0.444 \\
\hline hsa-miR-497-5p & 0.922 & 0.033 & 0.444 \\
\hline hsa-miR-543 & -1.587 & 0.034 & 0.444 \\
\hline hsa-miR-6500-5p & -1.783 & 0.024 & 0.394 \\
\hline hsa-miR-6529-3p & -2.703 & 0.002 & 0.271 \\
\hline hsa-miR-6729-5p & 2.238 & 0.008 & 0.368 \\
\hline hsa-miR-6869-5p & -2.122 & 0.014 & 0.368 \\
\hline hsa-miR-7704 & -1.595 & 0.018 & 0.368 \\
\hline hsa-miR-9851-3p & 2.304 & 0.006 & 0.331 \\
\hline & & & \\
\hline
\end{tabular}


Table 5.4: Significantly enriched KEGG pathways

\begin{tabular}{|c|c|c|c|c|}
\hline KEGG pathway & $\begin{array}{l}\text { Number of } \\
\text { genes tested }\end{array}$ & $\log \mathrm{FC}$ & $\begin{array}{l}\text { Standard } \\
\text { Error }\end{array}$ & $\begin{array}{l}\text { FDR-adjusted } p \\
\text { value }\end{array}$ \\
\hline Pathways in cancer & 324 & -0.121 & 0.014 & 0.000 \\
\hline p53 signaling pathway & 68 & -0.184 & 0.027 & 0.000 \\
\hline Cell cycle & 123 & -0.148 & 0.021 & 0.000 \\
\hline Neurotrophin signaling pathway & 126 & -0.145 & 0.021 & 0.000 \\
\hline Prostate cancer & 89 & -0.168 & 0.025 & 0.000 \\
\hline Chronic myeloid leukemia & 73 & -0.174 & 0.027 & 0.000 \\
\hline Glioma & 65 & -0.182 & 0.029 & 0.000 \\
\hline Focal adhesion & 199 & -0.112 & 0.018 & 0.000 \\
\hline Bladder cancer & 42 & -0.207 & 0.035 & 0.000 \\
\hline Olfactory transduction & 149 & 0.166 & 0.031 & 0.000 \\
\hline Insulin signaling pathway & 134 & -0.117 & 0.022 & 0.000 \\
\hline Melanoma & 70 & -0.153 & 0.029 & 0.000 \\
\hline Small cell lung cancer & 84 & -0.140 & 0.027 & 0.000 \\
\hline Nod like receptor signaling pathway & 60 & -0.149 & 0.030 & 0.000 \\
\hline Mapk signaling pathway & 264 & -0.082 & 0.017 & 0.000 \\
\hline Tgf beta signaling pathway & 83 & -0.120 & 0.027 & 0.000 \\
\hline Leukocyte transendothelial migration & 113 & -0.105 & 0.024 & 0.000 \\
\hline Adherens junction & 72 & -0.121 & 0.029 & 0.000 \\
\hline Regulation of actin cytoskeleton & 207 & -0.078 & 0.019 & 0.000 \\
\hline Non small cell lung cancer & 54 & -0.136 & 0.034 & 0.001 \\
\hline Chemokine signaling pathway & 181 & -0.081 & 0.020 & 0.001 \\
\hline Renal cell carcinoma & 70 & -0.118 & 0.031 & 0.001 \\
\hline Colorectal cancer & 62 & -0.117 & 0.032 & 0.002 \\
\hline Neuroactive ligand receptor interaction & 252 & 0.080 & 0.022 & 0.003 \\
\hline Pancreatic cancer & 70 & -0.110 & 0.031 & 0.003 \\
\hline Acute myeloid leukemia & 57 & -0.117 & 0.033 & 0.003 \\
\hline $\begin{array}{l}\text { Epithelial cell signaling in helicobacter } \\
\text { pylori infection }\end{array}$ & 68 & -0.108 & 0.031 & 0.003 \\
\hline ECM receptor interaction & 84 & -0.095 & 0.029 & 0.006 \\
\hline Oocyte meiosis & 110 & -0.083 & 0.026 & 0.008 \\
\hline WNT signaling pathway & 150 & -0.073 & 0.023 & 0.008 \\
\hline Endometrial cancer & 52 & -0.111 & 0.036 & 0.012 \\
\hline Cytosolic DNA sensing pathway & 49 & -0.110 & 0.036 & 0.012 \\
\hline Toll like receptor signaling pathway & 96 & -0.084 & 0.028 & 0.012 \\
\hline Spliceosome & 126 & -0.075 & 0.025 & 0.012 \\
\hline Lysosome & 121 & -0.075 & 0.025 & 0.015 \\
\hline Drug metabolism cytochrome $\mathrm{p} 450$ & 65 & 0.134 & 0.046 & 0.017 \\
\hline Nucleotide excision repair & 44 & 0.169 & 0.058 & 0.017 \\
\hline mTOR signaling pathway & 51 & -0.104 & 0.037 & 0.022 \\
\hline Type ii diabetes mellitus & 46 & -0.105 & 0.038 & 0.028 \\
\hline Base excision repair & 33 & 0.177 & 0.067 & 0.038 \\
\hline
\end{tabular}




\begin{tabular}{|l|l|l|l|l|}
\hline $\begin{array}{l}\text { Aldosterone regulated sodium } \\
\text { reabsorption }\end{array}$ & 41 & -0.106 & 0.041 & 0.041 \\
\hline Vibrio cholerae infection & 53 & -0.095 & 0.036 & 0.041 \\
\hline Erbb signaling pathway & 87 & -0.077 & 0.030 & 0.042 \\
\hline Adipocytokine signaling pathway & 65 & -0.085 & 0.033 & 0.044 \\
\hline RNA degradation & 56 & -0.089 & 0.036 & 0.049 \\
\hline
\end{tabular}

Table 5.5: Ten most positively and negatively enriched GO biological processes, out of 1033 significantly enriched GO biological processes.

\begin{tabular}{|l|l|l|l|l|}
\hline GO Biological Process & $\begin{array}{l}\text { Number of } \\
\text { genes tested }\end{array}$ & $\begin{array}{l}\text { Log2 Fold } \\
\text { change }\end{array}$ & $\begin{array}{l}\text { Standard } \\
\text { error }\end{array}$ & $\begin{array}{l}\text { FDR-adjusted } \\
\text { p value }\end{array}$ \\
\hline Intestinal epithelial cell development & 11 & -0.250 & 0.052 & 0.000 \\
\hline $\begin{array}{l}\text { Lymphoid progenitor cell } \\
\text { differentiation }\end{array}$ & 11 & -0.240 & 0.053 & 0.000 \\
\hline $\begin{array}{l}\text { Positive regulation of protein import } \\
\text { into nucleus translocation }\end{array}$ & 13 & -0.236 & 0.044 & 0.000 \\
\hline Pre mirna processing & 13 & -0.231 & 0.054 & 0.000 \\
\hline Endothelial tube morphogenesis & 11 & -0.228 & 0.060 & 0.001 \\
\hline $\begin{array}{l}\text { Positive regulation of transforming } \\
\text { growth factor beta production }\end{array}$ & 16 & -0.222 & 0.049 & 0.000 \\
\hline Mitotic sister chromatid cohesion & 12 & -0.220 & 0.051 & 0.000 \\
\hline $\begin{array}{l}\text { Regulation of nuclear transcribed } \\
\text { mRNA poly a tail shortening }\end{array}$ & 11 & -0.217 & 0.063 & 0.004 \\
\hline $\begin{array}{l}\text { Positive regulation of extrinsic } \\
\text { apoptotic signaling pathway via death } \\
\text { domain receptors }\end{array}$ & 17 & -0.216 & 0.049 & 0.000 \\
\hline B cell homeostasis & 21 & -0.216 & 0.042 & 0.000 \\
\hline $\begin{array}{l}\text { Sensory perception of chemical } \\
\text { stimulus }\end{array}$ & 200 & 0.154 & 0.027 & 0.000 \\
\hline $\begin{array}{l}\text { Phosphatidylcholine acyl chain } \\
\text { remodeling }\end{array}$ & 24 & 0.199 & 0.077 & 0.042 \\
\hline Reflex & 20 & 0.210 & 0.082 & 0.045 \\
\hline Lipid digestion & 19 & 0.236 & 0.088 & 0.036 \\
\hline Mismatch repair & 27 & 0.243 & 0.072 & 0.005 \\
\hline $\begin{array}{l}\text { Nucleotide excision repair pre- } \\
\text { incision complex stabilization }\end{array}$ & 21 & 0.249 & 0.082 & 0.014 \\
\hline $\begin{array}{l}\text { DNA dependent DNA replication } \\
\text { maintenance of fidelity }\end{array}$ & 24 & 0.251 & 0.076 & 0.006 \\
\hline $\begin{array}{l}\text { Detection of temperature stimulus } \\
\text { involved in sensory perception }\end{array}$ & 12 & 0.269 & 0.105 & 0.045 \\
\hline $\begin{array}{l}\text { Positive regulation of triglyceride } \\
\text { lipase activity }\end{array}$ & 10 & 0.337 & 0.090 & 0.002 \\
\hline Protein auto-processing & 13 & 0.108 & 0.048 \\
\hline
\end{tabular}


Table 5.6: Ten most positively and negatively enriched GO molecular function, out of 144 significantly enriched GO biological processes

\begin{tabular}{|l|l|l|l|l|}
\hline GO molecular function & $\begin{array}{l}\text { Number } \\
\text { of genes } \\
\text { tested }\end{array}$ & $\begin{array}{l}\text { Log2 } \\
\text { Fold } \\
\text { change }\end{array}$ & $\begin{array}{l}\text { Standard } \\
\text { error }\end{array}$ & $\begin{array}{l}\text { FDR- } \\
\text { adjusted } \\
\text { p value }\end{array}$ \\
\hline Insulin receptor substrate binding & 11 & -0.24 & 0.05 & 0.00 \\
\hline miRNA binding & 16 & -0.22 & 0.05 & 0.00 \\
\hline Phosphatase activator activity & 11 & -0.22 & 0.06 & 0.00 \\
\hline Nuclear localization sequence binding & 21 & -0.21 & 0.04 & 0.00 \\
\hline $\begin{array}{l}\text { Transcription factor activity rna polymerase } \\
\text { ii core promoter sequence specific }\end{array}$ & 13 & -0.20 & 0.05 & 0.00 \\
\hline Translation repressor activity & 19 & -0.20 & 0.05 & 0.00 \\
\hline Activin binding & 12 & -0.19 & 0.05 & 0.01 \\
\hline Pre-mRNA binding & 24 & -0.19 & 0.04 & 0.00 \\
\hline Transcription cofactor binding & 24 & -0.19 & 0.04 & 0.00 \\
\hline Ubiquitin ubiquitin ligase activity & 13 & -0.19 & 0.06 & 0.02 \\
\hline Cation channel activity & 279 & 0.09 & 0.02 & 0.00 \\
\hline Voltage gated cation channel activity & 130 & 0.09 & 0.03 & 0.03 \\
\hline G protein coupled receptor activity & 515 & 0.11 & 0.02 & 0.00 \\
\hline Delayed rectifier potassium channel activity & 36 & 0.17 & 0.06 & 0.05 \\
\hline Taste receptor activity & 20 & 0.22 & 0.08 & 0.03 \\
\hline Olfactory receptor activity & 121 & 0.23 & 0.03 & 0.00 \\
\hline Quinone binding & 17 & 0.26 & 0.09 & 0.02 \\
\hline Odorant binding & 23 & 0.28 & 0.07 & 0.00 \\
\hline $\begin{array}{l}\text { Bicarbonate transmembrane transporter } \\
\text { activity }\end{array}$ & 17 & 0.33 & 0.08 & 0.00 \\
\hline Mismatched DNA binding & 12 & 0.34 & 0.09 & 0.00 \\
\hline & & & & \\
\hline
\end{tabular}




\subsection{Figures}

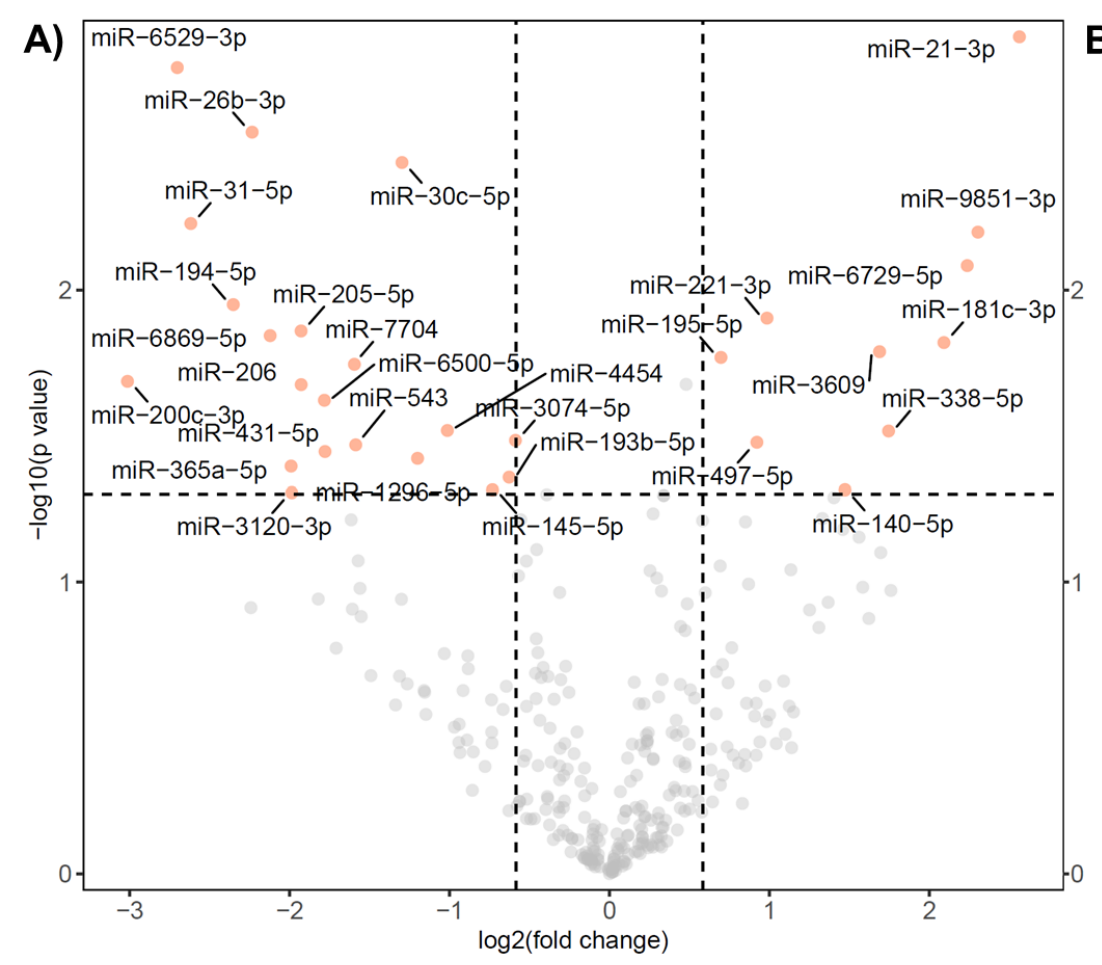

B)
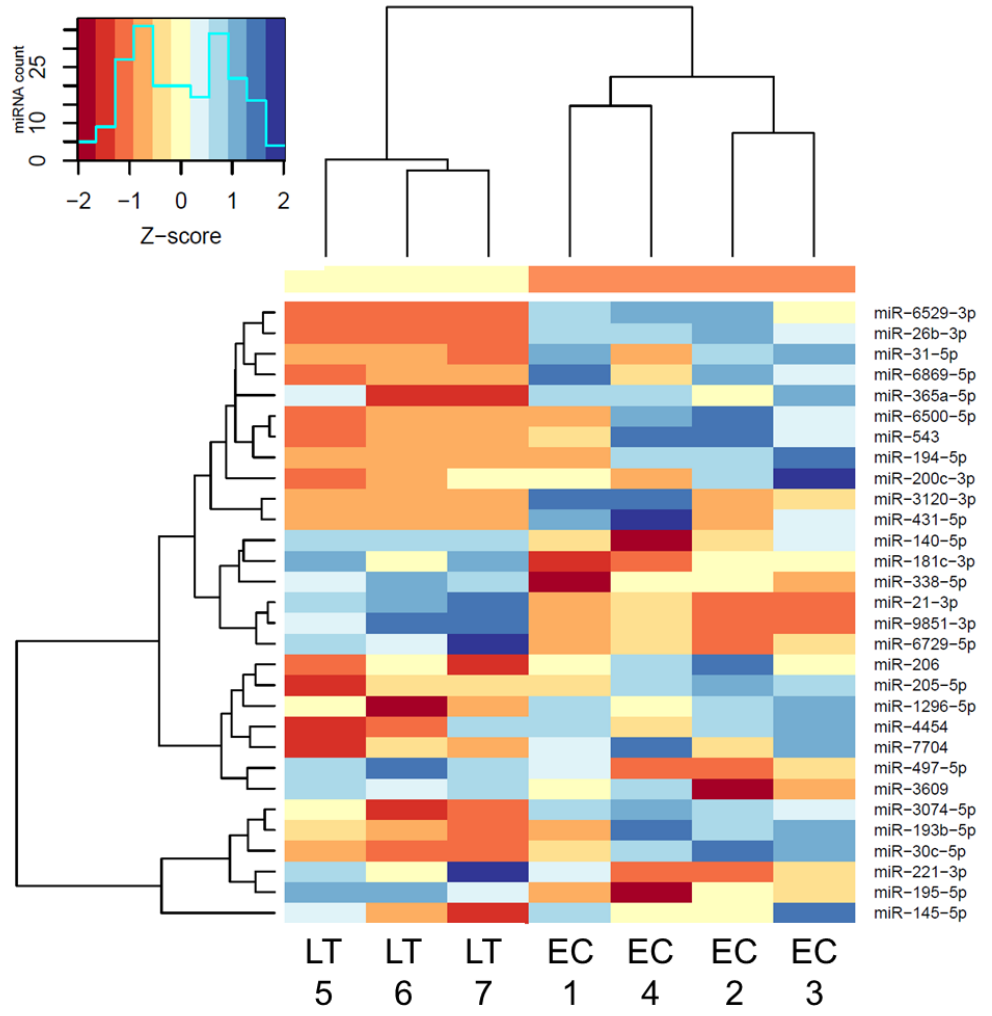

Figure 5.1: Enriched microRNAs from LT 13-lined ground squirrel WAT, compared to EC. A) Volcano plot showing log2-transformed foldchanges in gene expression and $\log 10$-transformed p-values, where dotted lines show the thresholds of statistical significance for each metric (significant if fold changes are $>1.5$-fold, p-value $<0.05$ ). B) Supervised cluster analysis showing similarity of miRNA CLR read counts as rows, and similarity of samples shows in columns. The sample WAT8 was relabeled as LT7. Inset histogram shows CLR read count distribution. 
A)

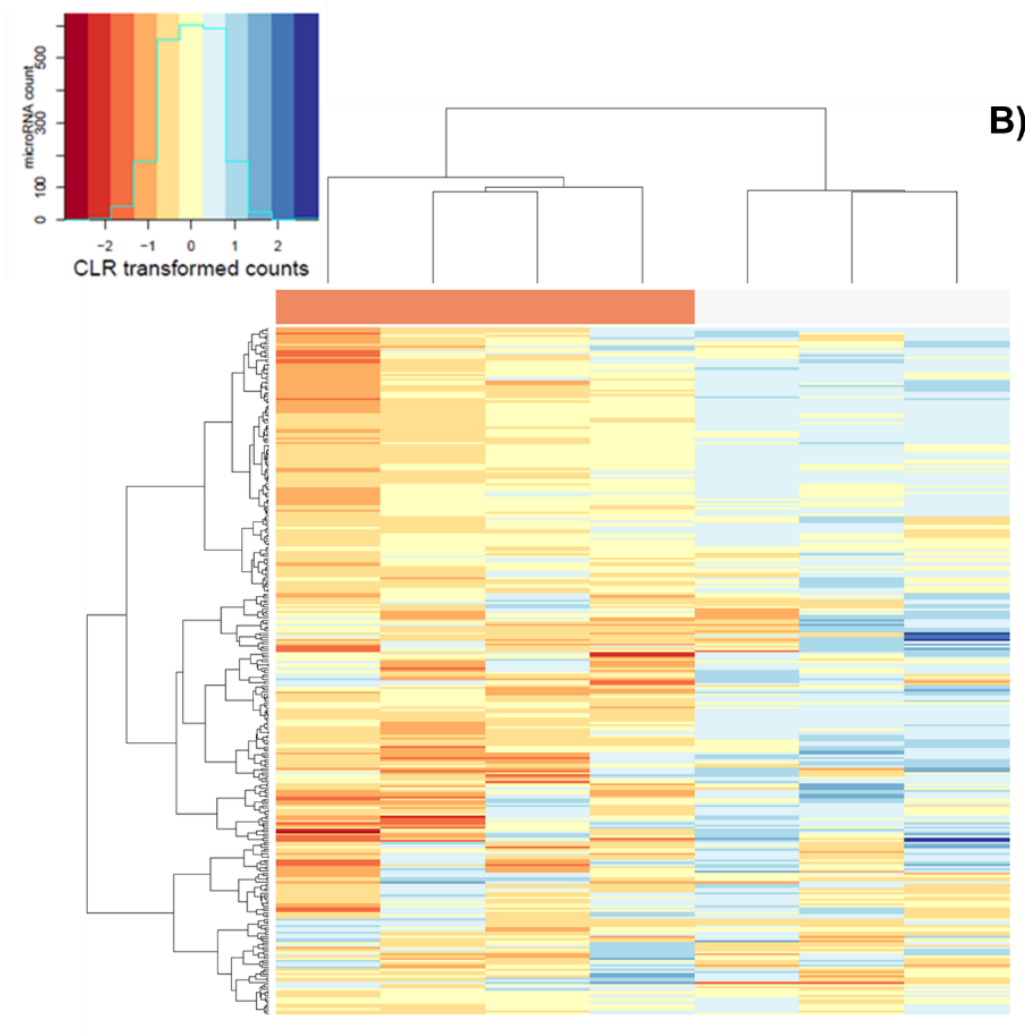

EC EC LT LT LT EC EC
B)

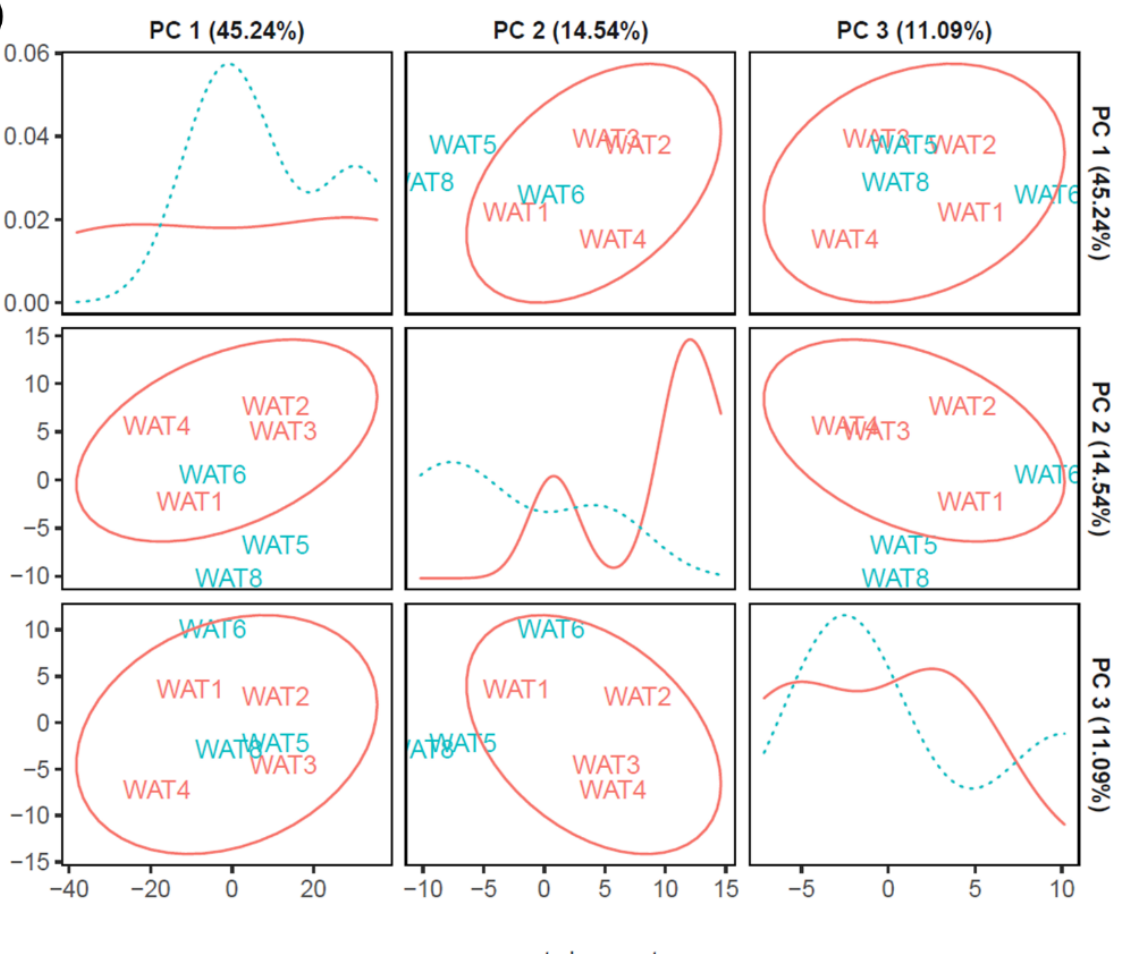

Figure 5.2: Results of unsupervised clustering analysis and principle component analysis (PCA) of samples based on relative miRNA levels comparing EC and LT. A) Heatmap of unsupervised cluster analysis of differentially expressed genes and B) PCA biplots showing relationship between different principal components PC1, PC2, or PC3, and density plots are shown on the left to right diagonal. 


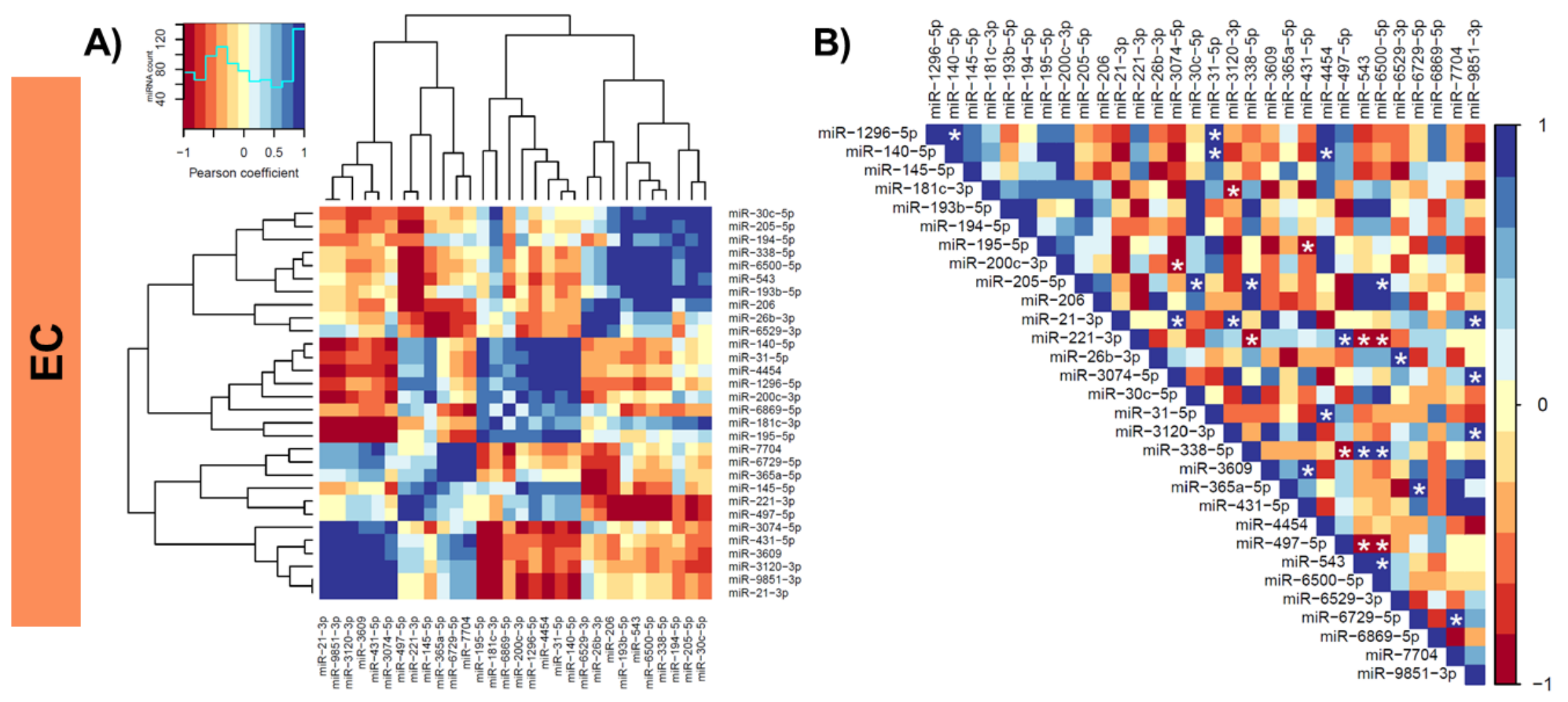

Figure 5.3: Supervised Pearson correlation plots of differentially expressed microRNAs $(p<0.05)$, showing miRNAs that are co-expressed during in WAT of euthermic in the cold room control animals (EC). Stars on the correlation plots (B) indicate statistical significance $(\mathrm{p}<0.05)$, where dark blue are co-expressions and dark red signify opposite miRNA expression patterns. 

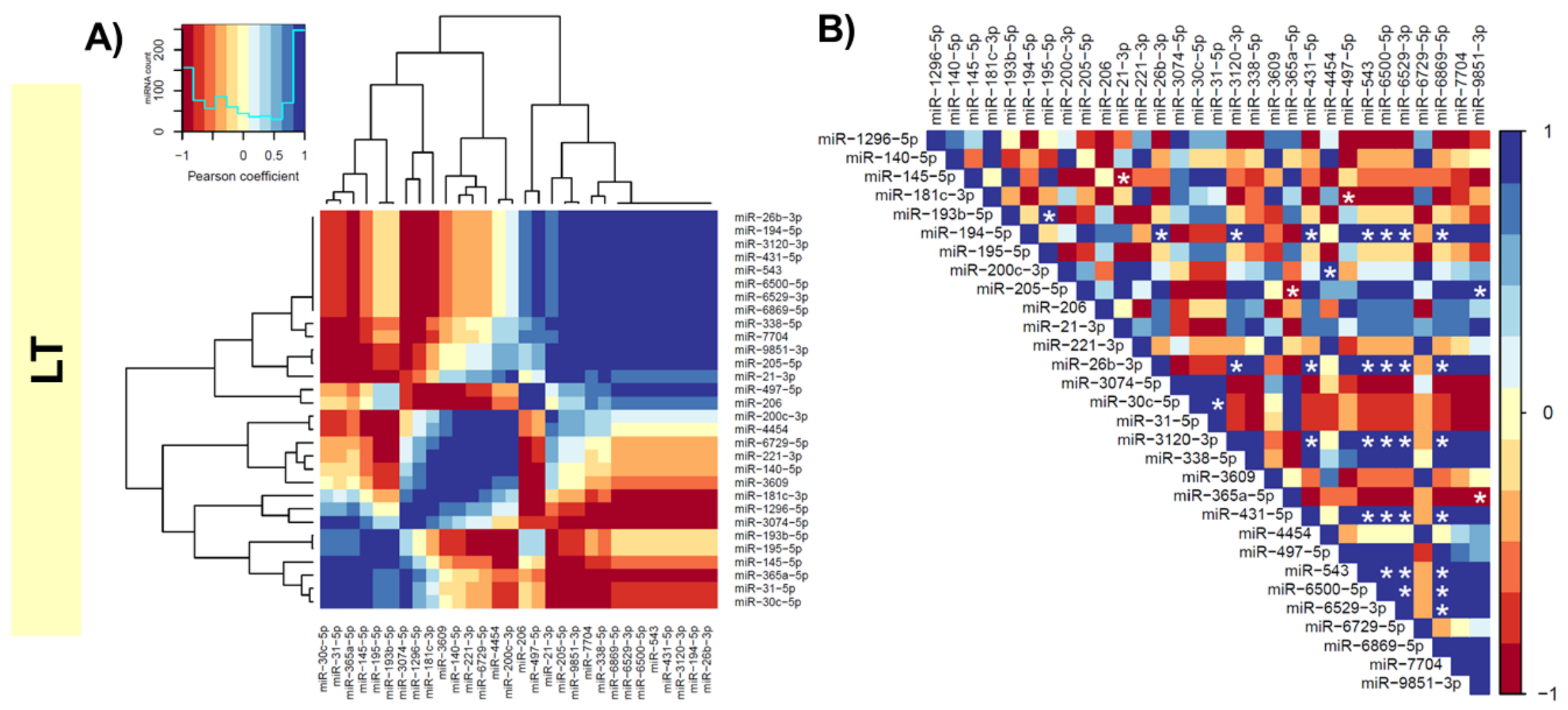

Figure 5.4: Supervised Pearson correlation plots of differentially expressed microRNAs $(p<0.05)$, showing miRNAs that are co-expressed during torpor (LT). Stars on the correlation plot $(\mathrm{B})$ indicate statistical significance $(\mathrm{p}<0.05)$, where dark blue are co-expressions and dark red signify opposite miRNA expression patterns. 

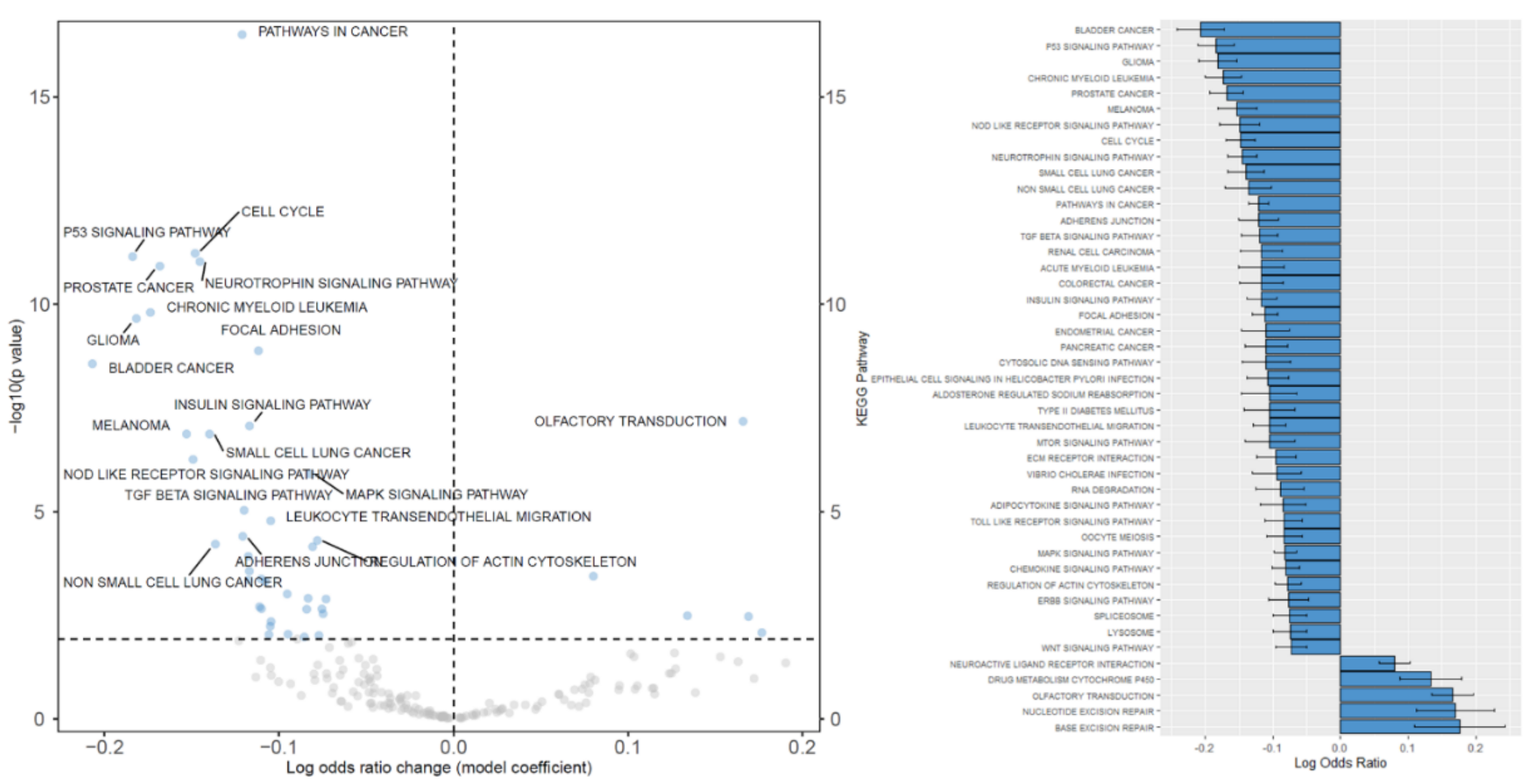

Figure 5.5: Significantly enriched KEGG pathways using an FDR-adjusted p-values $<0.05$, where pathways are shown in A) a volcano plot showing logarithmically transformed model coefficients and - $\log 10$ transformed p-values and B) a histogram of the forty-five enriched KEGG pathways with standard error as error bars. 

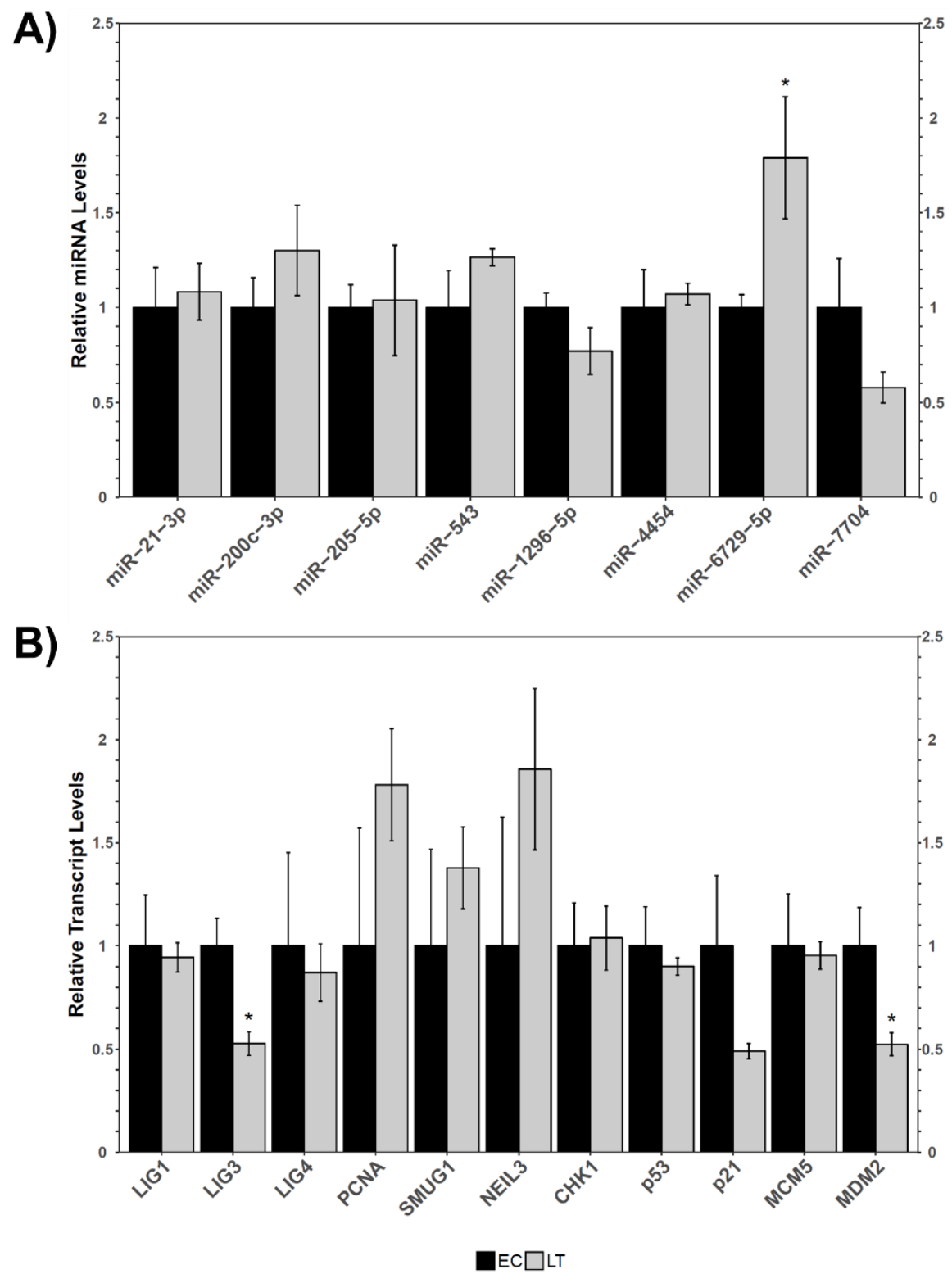

Figure 5.6: RT-qPCR of miRNA-seq and downstream target genes in 13-lined ground squirrel WAT, comparing EC and LT. A) Relative miRNA levels of 8 miRNAs proposed to be differentially regulated according to miRNA-seq, using the geometric mean of TMET and snord75 as the reference genes. B) Relative mRNA transcript levels that may be targeted by differentially regulated microRNAs. The geometric mean of the Normfinder-identified pair of genes, GNB and PPIA, was used as the reference gene. Mean expression (+/- standard error) are shown for each gene. For miRNA analysis an $n=5$ biological replicates per condition was used except an $\mathrm{n}=4$ was used for torpor for miRNAs miR-543, miR-1296-5p, miR-4454, and miR7704. For mRNA analysis, $n=4$ biological replicates per condition were used except $n=3$ were used for torpor in LIG1 analysis and for control in NEIL3 analysis. 


\section{A)}

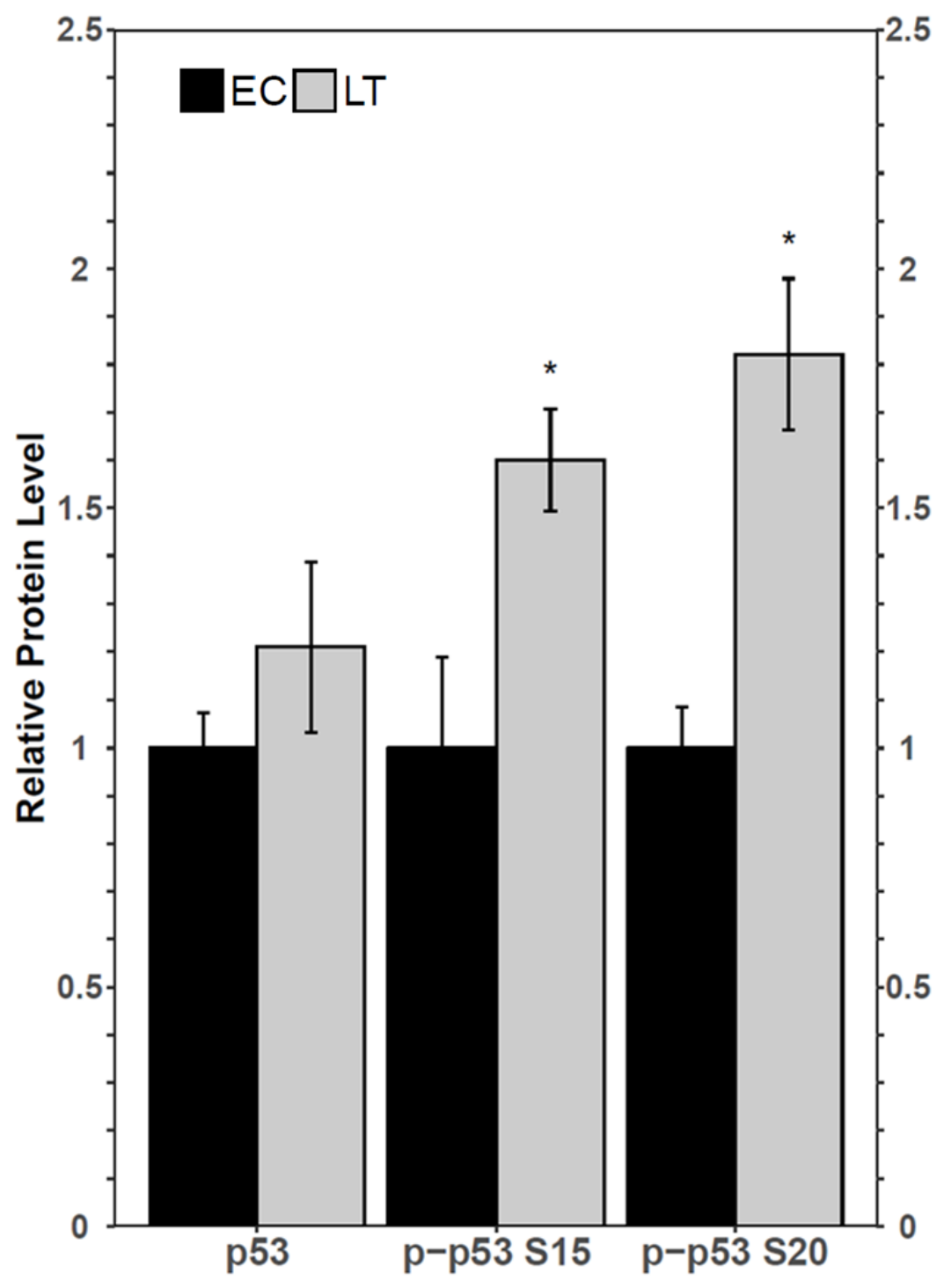

B)

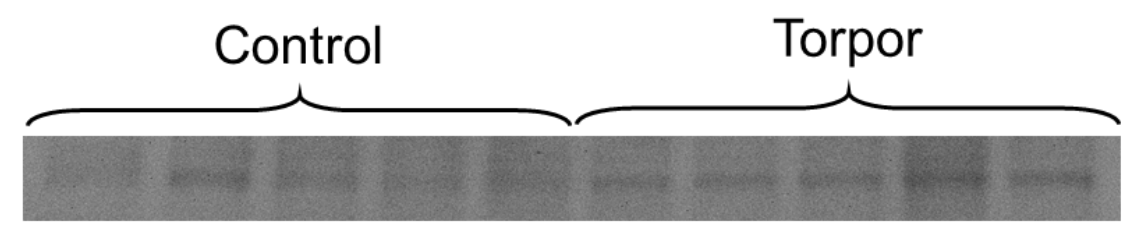

p-p53 S15

p-p53 S20

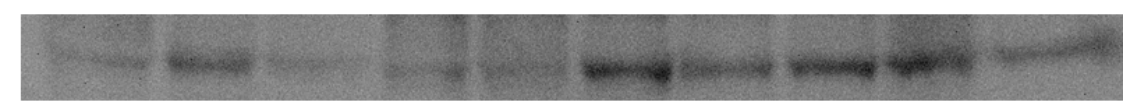

Figure 5.7: Relative protein levels of $\mathrm{p} 53$ protein and $\mathrm{p} 53$ phosphorylation markers in white adipose tissue comparing EC and LT ground squirrels. A) Histogram showing relative protein expression, analyzed by a Student's t-test where an asterisk indicates statistical significance $(\mathrm{p}<$ $0.05)$. B) Representative bands showing $\mathrm{n}=5$ biological replicates per condition for control and torpor for each $\mathrm{p} 53$ protein or phosphorylation site. 


\subsection{Supplemental Figures}
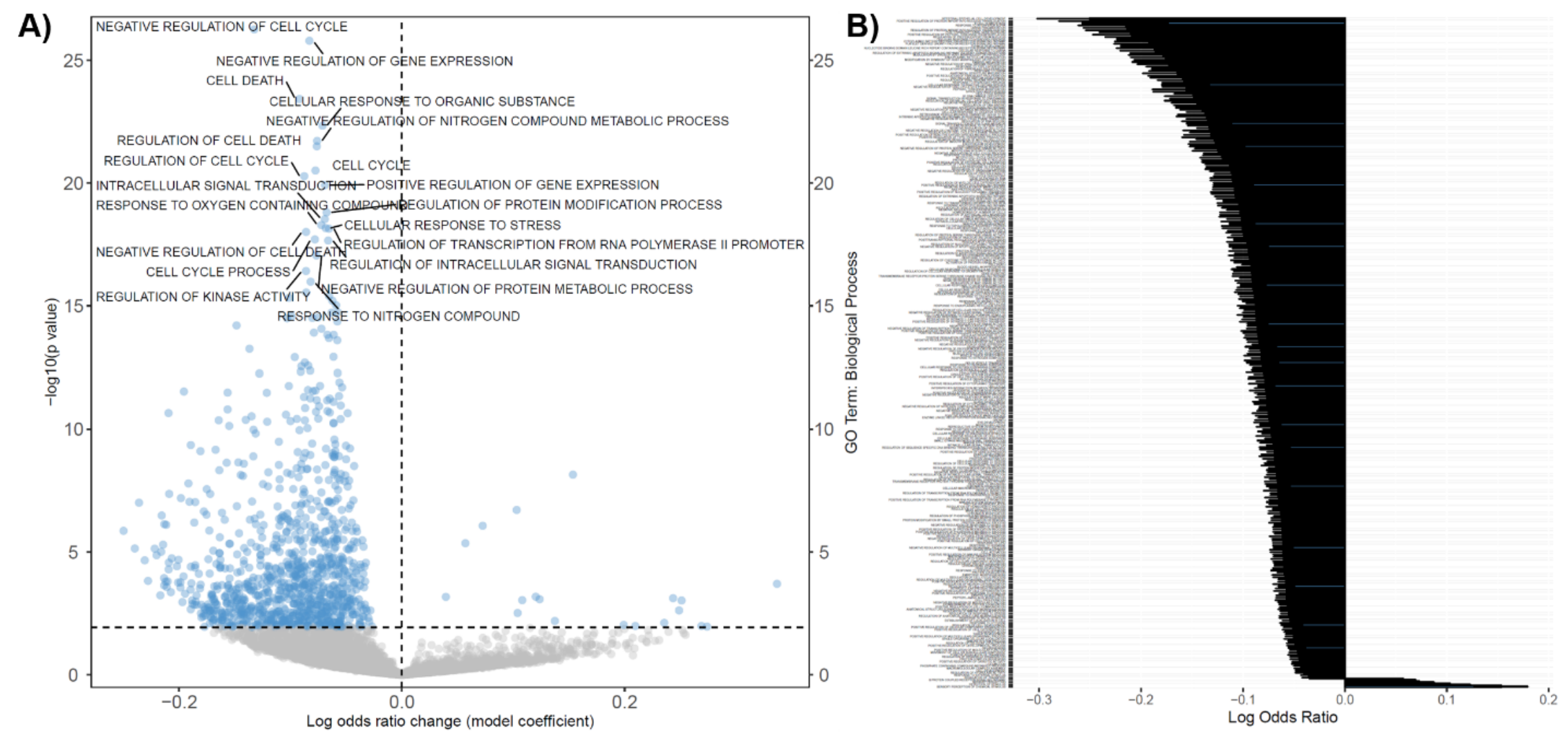

Supplemental Figure 5.1: Significantly enriched GO biological processes (FDR-adjusted P-values $<0.05$ ). A) Volcano plot shows all GO BPs that are differentially enriched in relation to their $\log 10$ transformed $\mathrm{p}$-value and $\mathrm{B}$ ) the histogram shows the log-fold change in the model coefficient along with its standard error for GO BPs with FDR-adjusted $\mathrm{p}<0.0001$ 


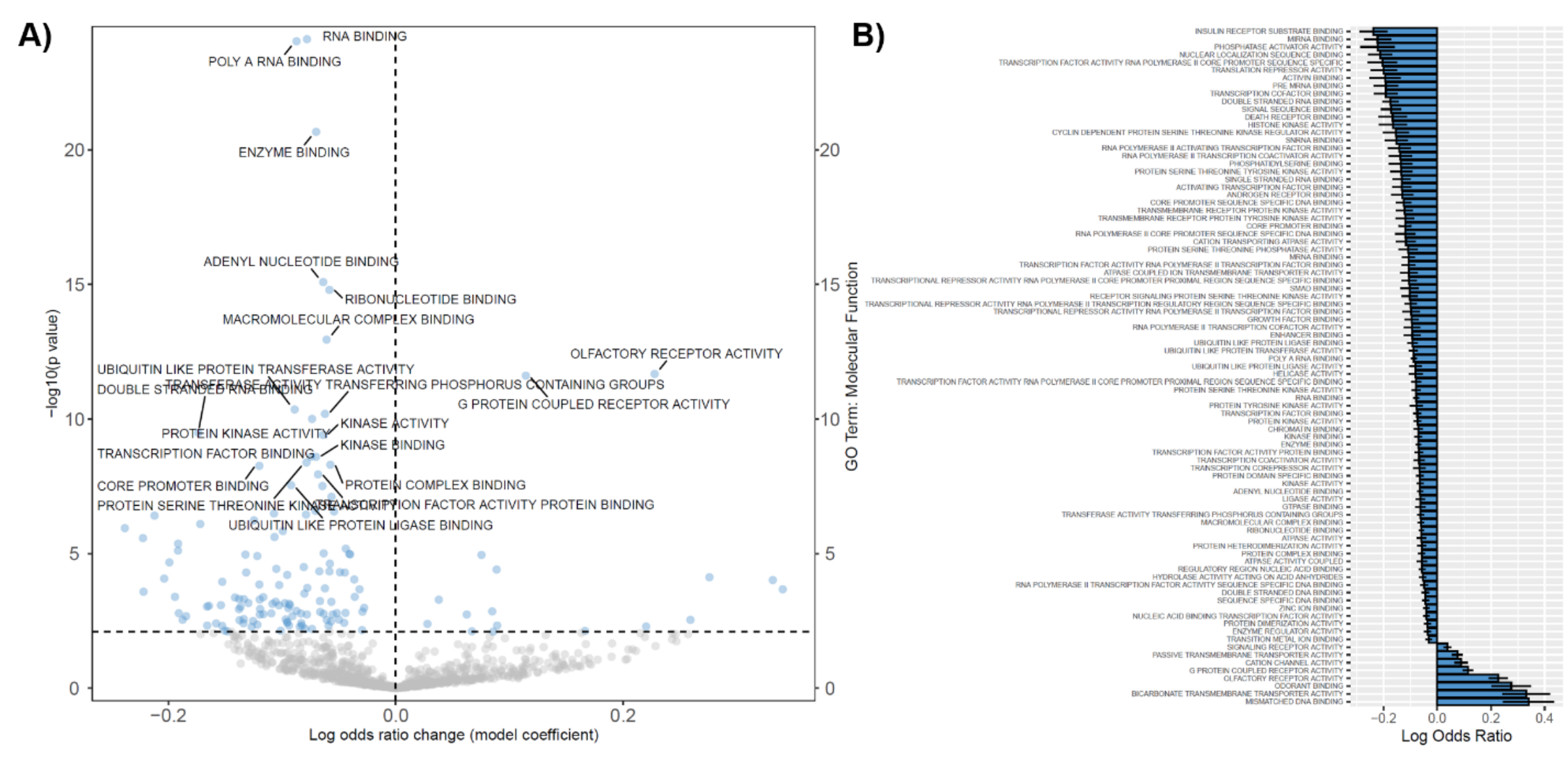

Supplemental Figure 5.2: Significantly enriched GO molecular functions (FDR-adjusted P-values $<0.05$ ). A) Volcano plot shows all GO MFs that are differentially enriched in relation to their $\log 10$ transformed p-value and B) the histogram shows the log-fold change in the model coefficient along with its standard error for GO BPs with FDR-adjusted $\mathrm{p}<0.01$. 


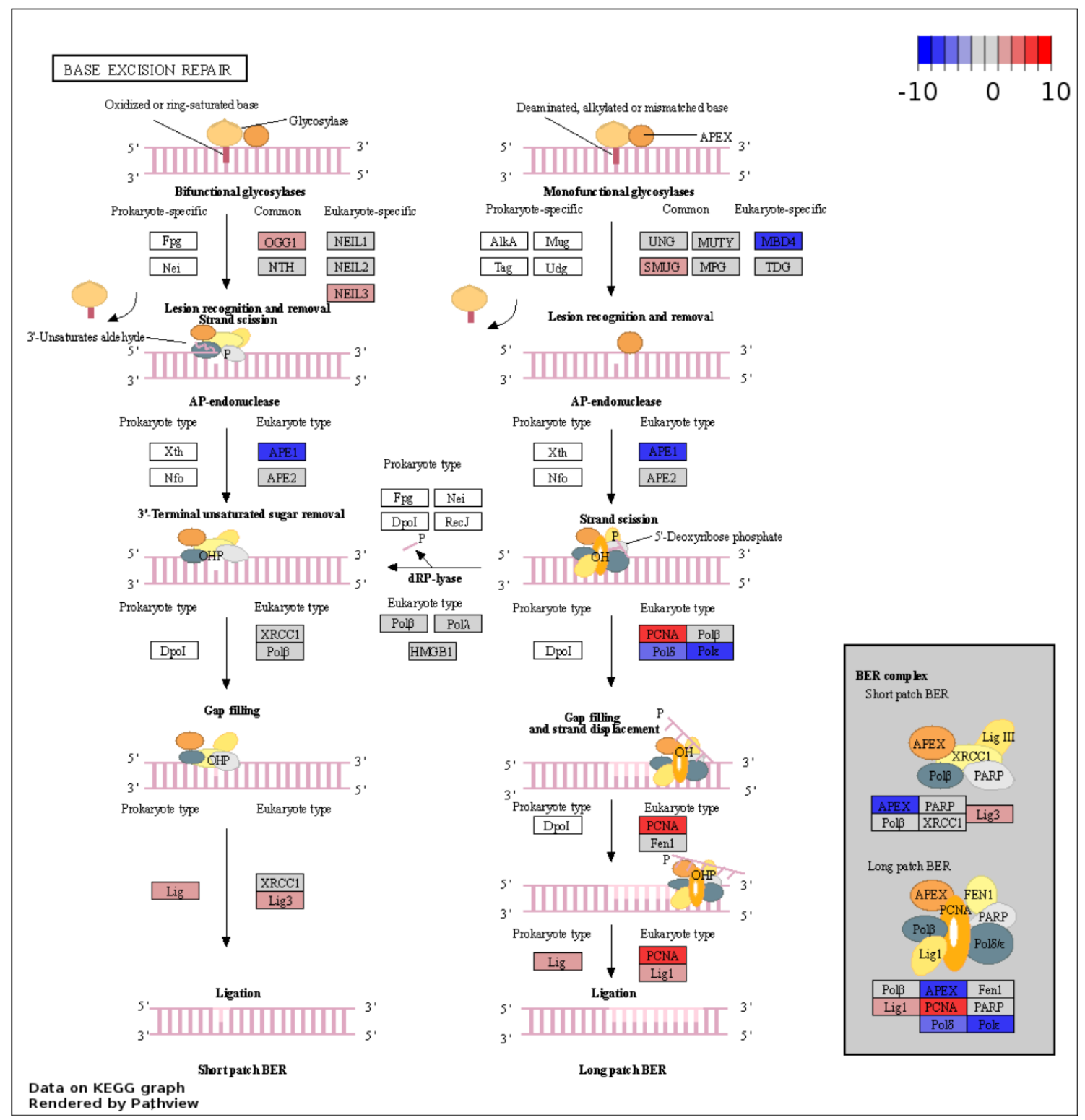

Supplemental Figure 5.3: Pathview analysis of Base Excision Repair KEGG Pathway. Genes that may be downregulated during LT by miRNAs are shown in blue and genes that are less inhibited by miRNAs during LT are shown in red. 


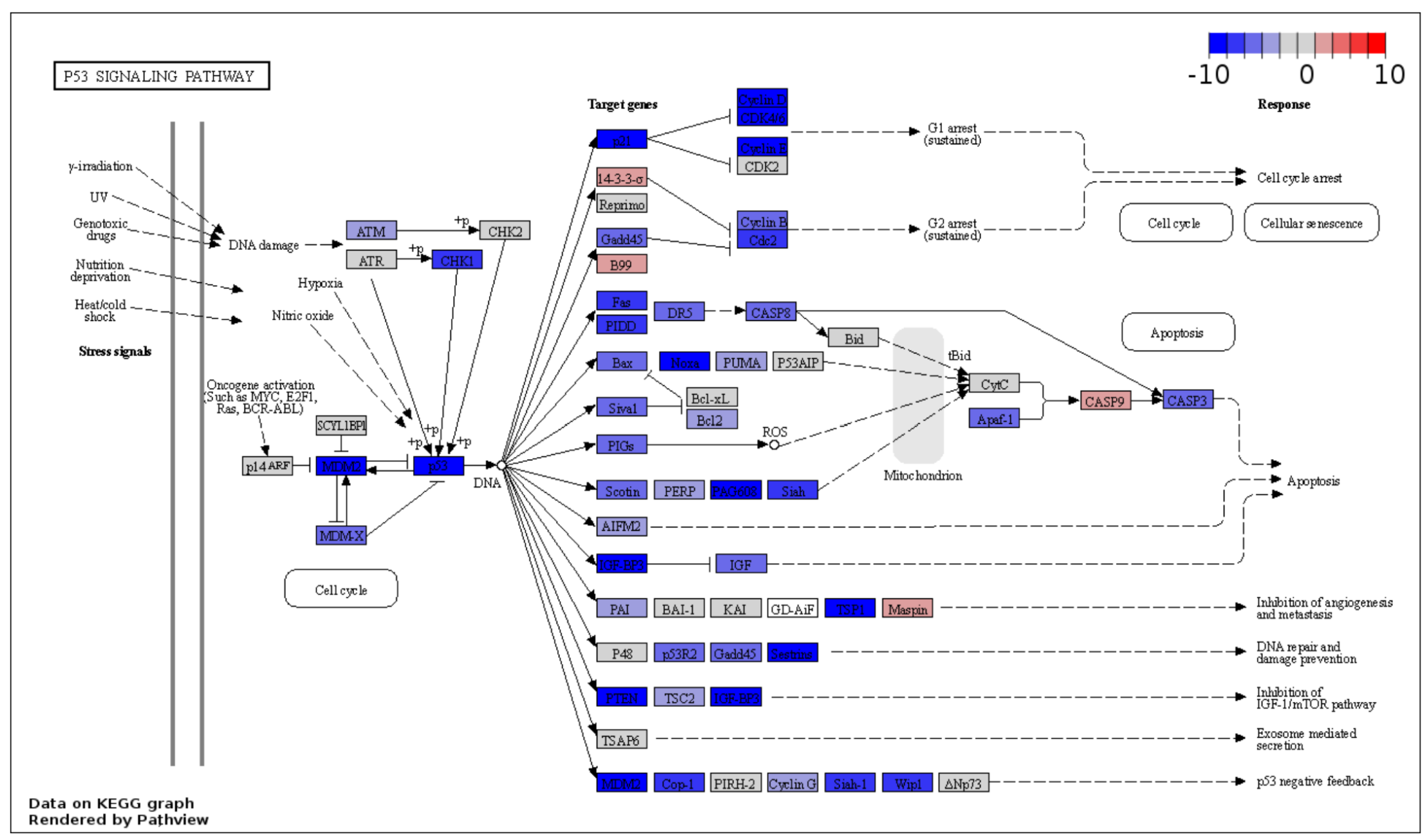

Supplemental Figure 5.4: Pathview analysis of p53 signaling pathway. Genes that may be downregulated during LT by miRNAs are shown in blue and genes that are less inhibited by miRNAs during LT are shown in red. 


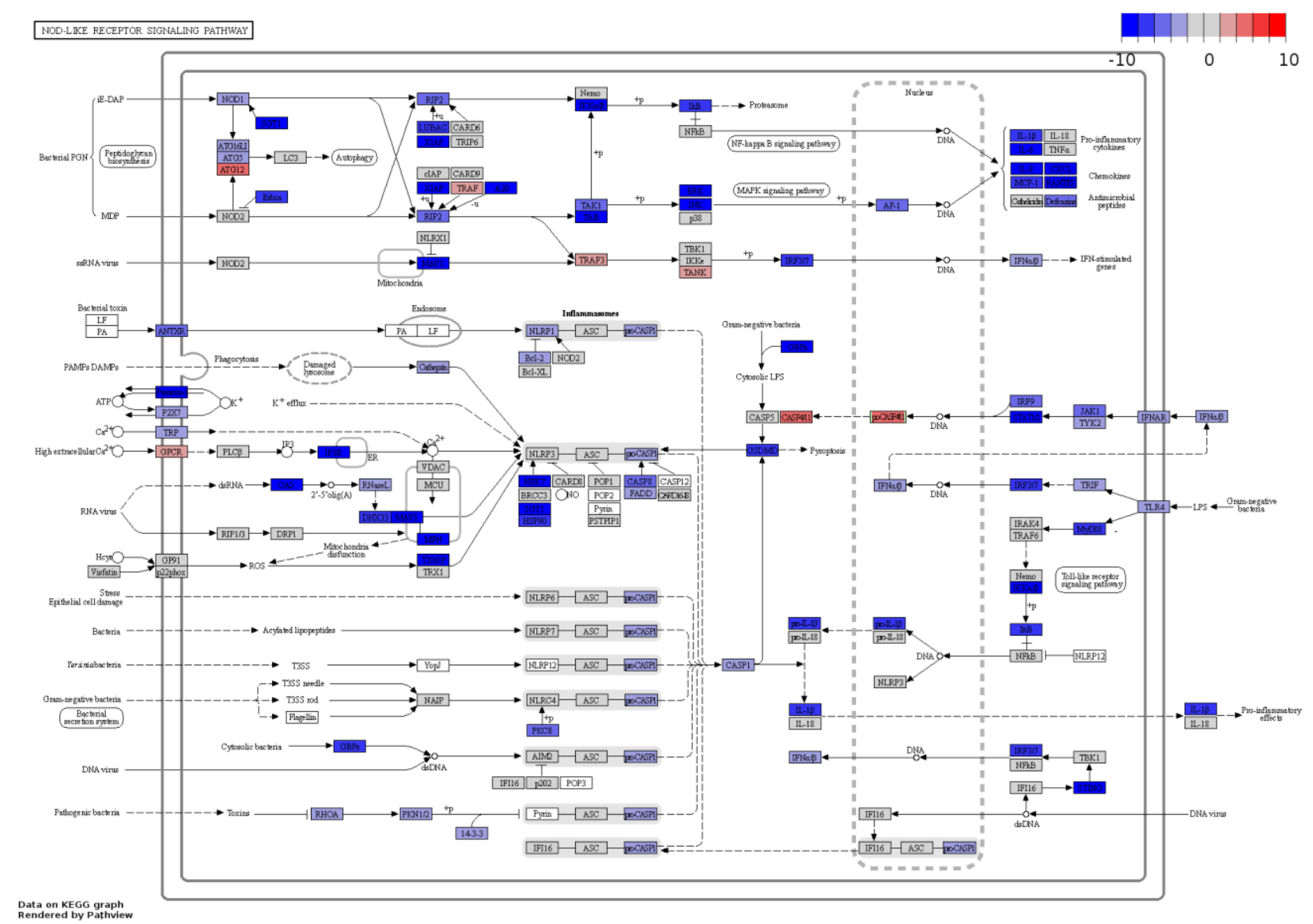

Supplemental Figure 5.5: Pathview analysis of NOD-like receptor signaling pathway. Genes that may be downregulated during LT by miRNAs are shown in blue and genes that are less inhibited by miRNAs during LT are shown in red. 


\section{General Discussion}




\subsection{Discussion}

Hibernators are highly adaptable species that must make intricate and coordinated changes at the molecular level to preserve metabolic fuel/energy and avoid tissue damage, both very important in maintaining whole-body health and viability over long winter months in torpor. Reductions in energy expenditure can save small mammals up to $99 \%$ of the energy they would have expended if they remained euthermic throughout the winter (Storey, 2010). Even though obligate hibernators like the 13-lined ground squirrel remain immobile for months at a time, they do not show signs of significant muscle atrophy. Furthermore, hibernators typically tolerate unnatural stressors better if they are allowed to enter torpor. Hibernating ground squirrels resist damage from ischemia and reperfusion surgery more than active ground squirrels, based on lower post-reperfusion levels of biomarkers indicative of liver damage and lower levels of double stranded DNA breaks indicative of apoptosis (Kurtz et al., 2006). Winter hibernating 13-lined ground squirrels also tolerate higher levels of ${ }^{60} \mathrm{Co}$ gamma rays compared to winter active ground squirrels (Musacchia and Barr, 1968). All hibernating ground squirrels survived 30 days after receiving 1100 and 1250 rads of radiation, but only $67 \%$ and $0 \%$ of winter active ground squirrels survived these doses ${ }^{1}$.

The behavioural, physiological, and molecular adaptations hibernators use to survive extreme environments are not only of interest to curious minds who study them for the sake of their peculiarity, or the advancement of the field of comparative biology. Understanding the mechanisms of mammalian hibernation is important to advance the science behind long-term cold storage of organs for biobanking or human transplants. The keys to hibernation may unlock a world where long term spaceflight to distant galaxies becomes possible, based on an understanding of the ways that hibernators can enter reversible dormancy, minimize energy

\footnotetext{
${ }^{1}$ It should be noted that ground squirrels have been reported to die during the winter if they cannot enter torpor.
} 
usage, prevent muscle wasting despite chronic immobility, and reduce tissue damage from radiation.

In this thesis, I focused on white adipose tissue (WAT) and brown adipose tissue (BAT) because hibernating ground squirrels are touted as being "fat-but-fit". They don't show any longlasting symptoms of chronic obesity, despite doubling their weight during the fall period of hyperphagia and maintaining a high fat mass for months (MacCannell et al., 2017). Both adipose depots serve as important regulators of whole-body homeostasis during torpor, although BAT is more metabolically active than WAT, given that its role is in rewarming the animal from $5-10{ }^{\circ} \mathrm{C}$ during torpor back to euthermic levels. WAT provides an essential role in the storage of triglycerides, the main source of cellular fuel throughout winter torpor.

The studies within this thesis were inspired by the idea that chronically obese hibernators must have mechanisms to curb adipose dysfunction (unlike what we see in non-hibernators), especially given that previous studies on hibernator adipose tissues show evidence of oxidative stress and possible activation of the innate immune system at different time points of the torporarousal cycle. For instance, increased markers of lipid peroxidation and protein carbonylation are present in BAT of aroused ground squirrels relative to torpor, and receptors for DAMP signaling such as RAGE are increased during torpor and arousal in BAT and WAT (Logan and Storey, 2018; Orr et al., 2009). These studies suggested that pro-inflammatory signaling may occur in BAT and/or WAT. The experiments performed in herein were done to investigate 1) if inflammasomes capable of sensing and responding to endogenous DAMPs are activated, 2) if the expression of cytokines and/or tissue remodeling proteins are altered, and 3) if miRNAs could regulate metabolic pathways in adipose tissues, and if so, which pathways. 


\subsubsection{Investigation of the regulation of the inflammasome}

Chapter 2 explored the regulation of the inflammasome, a highly complex pro-

inflammatory pathway that requires several key events for activation. It was expected that the inflammasome would largely be activated upon arousal, when breathing rate, metabolic rate and non-shivering thermogenesis increase, possibly creating ROS and other pro-inflammatory molecules (Orr et al., 2009). The results of previous studies also suggested that the inflammasome could be increased during torpor. For example, RAGE protein levels increased in both WAT and BAT during torpor and arousal, and if triggered by a DAMP, this event could theoretically increase NFkB expression and activity (Logan and Storey, 2018). NFkB may be activated in torpid ground squirrel BAT and WAT, given increased phosphorylation of IкB $\alpha$ and increased protein levels of a key gene downstream of NFאB activation (Hadj-Moussa et al., 2020).

The experiments in this thesis probed the regulation of inflammasome activation by looking at markers of inflammasome priming. RT-qPCR experiments were instrumental to determine how mRNA transcription of key inflammasome genes change over the torpor-arousal cycle and helped determine when "priming" of the NLRP3 inflammasome may occur in hibernators. Consistency in the temporal upregulation of NLRP3, Casp1, and IL-1 $\beta$ gene expression in BAT suggested that priming may occur during interbout arousal. Cell stress may be detected by TLRs at this time and activate transcription factors like NFKB that can increase proinflammatory gene expression, including the expression of NLRP3, Casp1 and IL-1 $\beta$. The idea that pro-inflammatory gene expression may increase in BAT upon arousal was consistent with the findings from hamster brain, which showed an increase in IL-1 $\beta$ and IL-6 mRNA levels during early arousal from torpor (Cogut et al., 2017). Translation of NLRP3 appeared to take place during torpor, whereas Aim 2 increased upon arousal (relative to torpor). Cleavage of pro-IL-1 $\beta$ may occur during torpor, based on low IL-1 $\beta$ levels observed via western blots. Combined with the finding that total IL- $1 \beta$ levels are generally higher during early arousal, these results suggest the NLRP3 or Aim2 inflammasomes may be activated during torpor or arousal, respectively, to 
increase caspase- 1 activity and IL- $1 \beta$ processing. Finally, the increase in BAT caspase- 1 activity during arousal further supports a role for inflammasome activation in ground squirrels upon rewarming.

Increases in RAGE and markers of NFאB activation in WAT also suggested that inflammasome priming could be possible during torpor. However, Chapter 2 reported few changes in inflammasome gene expression in ground squirrel WAT but the most important biomarker of inflammasome activation, caspase-1 activity, was significantly downregulated in both torpor and early arousal. Although RT-qPCR analyses revealed increases in ASC, GSDMD and IL-18, there were no increases in NLRP3 or IL-1 $\beta$. However, if sample size had been larger, it is possible that there could have been enough statistical power to detect a change in NLRP3 levels during ET, for example. Thus, genes whose levels did not change should be treated as inconclusive. In the future, if these results are to be validated in a larger study, an a priori power analysis should be conducted (using the results of this pilot study) to identify the sample size per group needed to detect a change when there is one to detect. Alternatively, if hibernators were limited as they were in the current study, perhaps future studies could use more sensitive approaches for the determination of relative protein levels, such as mass spectrometry. It would be interesting to conduct a proteome profiling study on WAT to confirm that key inflammasome genes are downregulated during torpor at the protein level. Otherwise, subsequent studies may investigate how pyrin-containing proteins (POPs) and card-containing proteins (COPs), decoy binding partners for some inflammasome receptors and caspases, respectively, may be used during torpor to curb innate immune pathways until spring euthermia.

\subsubsection{Evaluation of Objective 1}

\section{Determine if ground squirrel adipose tissues sense and respond to changes in cellular} homeostasis as they transition into and out of a torpor bout. 
Using western blotting, RT-qPCR, ELISA and enzyme activity assays, I determined that inflammasomes are likely activated during torpor and arousal in the BAT of 13-lined ground squirrels in response to cellular perturbation. Inflammasomes are unlikely to be activated in WAT based on the decrease in caspase-1 activity, but additional experiments using an increased sample size would be valuable to confirm this result. This study sets the stage for future experiments looking to characterize the importance of inflammatory and endocrine signals emitted from adipose tissues in hypometabolic animals.

\subsection{Differential regulation of pro-inflammatory and tissue remodeling proteins}

Chapter 3 built on the exploration of inflammatory signaling in the adipose tissues of a model hibernator by investigating the relative expression levels of key cytokines and modulators of the extracellular matrix. Cytokines, MMPs, and their negative regulators were measured in WAT and BAT due to their importance as biomarkers for inflammation and adipose tissue dysfunction. Generally, there were not many changes in the levels of cytokines, their soluble (decoy) receptors, MMPs, or TIMPs during torpor or early arousal. To recap, these two time points were chosen based on the idea that inflammation may be differentially regulated during torpor or early arousal, as some data from Chapter 1 had indicated, and because markers of damage, damage sensing, anti-apoptosis, and antioxidants increases in late torpid and early aroused ground squirrels.

One interesting finding in Chapter 3 was the increase in IL-1 $\alpha$ during torpor in ground squirrel BAT. The data from Chapter 2 (where IL-1 $\beta$ and caspase-1 activity increased in BAT during arousal) and increased IL-1 $\alpha$ levels suggested IL-1R signaling may have an important regulatory role at various time points of the torpor-arousal cycle. Both IL-1 subtypes signal through IL-1R. IL-1 $\alpha$ may be upregulated instead of IL-1 $\beta$ during torpor to invoke IL-1R 
signaling quickly, because unlike IL-1 $\beta$, it does not need inflammasome activation for processing, excretion, and activation. The lack of increases in IL-1ra, sIL-1RI, or sIL-1RII suggested that IL1 signaling may be uninhibited during torpor and arousal, but again, it would be interesting to confirm these results in a study with larger sample sizes per condition.

IL-1R signaling can lead to the activation of the transcription factors NFאB, AP-1, and cJun, which in turn, can increase levels of pro-inflammatory cytokines and regulators of innate immune cell differentiation. Briefly, when IL-1 binds IL-1R, it associates with the IL-1 receptor accessory protein (IL-1RAcP). The receptor complex recruits myeloid differentiation primary response protein 88 (MyD88), IL-1 receptor-associated kinase 1 (IRAK1), Tollip, and TNF receptor-associated factor 6 (TRAF6). Autophosphorylation of IRAK1 leads to its dissociation from the receptor complex, along with TRAF6. TRAF6 then associates with transforming growth factor b-activated kinase 1 (TAK1) and TAK1-binding (TAB) proteins. IRAK1 polyubiquitination results in its dissociation from the TRAF6-TAK1-TAB protein complex and it is either degraded or it remains at the membrane and interacts with other proteins like NF-kappaB essential modulator (NEMO). The TRAF6 complex then localizes to the cytoplasm where it associates with an E2 ubiquitin conjugating enzyme complex and become polyubiquitinated at lysine 63 . This chain of events leads to TAB2 binding to TAK1, resulting in its activation through phosphorylation and the phosphorylation of the NFאB mediator, IKK $\beta$. IKK $\alpha / \beta$-mediated

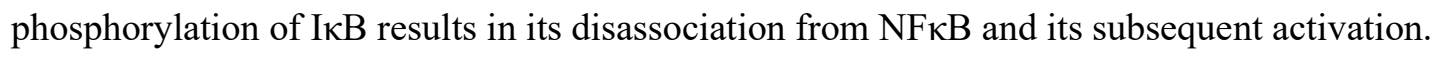
There has been little exploration of the signaling pathway downstream of IL-1R heterodimerization but recent evidence that suggests that $\mathrm{NF \kappa B}$ is less inhibited during torpor in WAT and BAT, so there is reason to believe that IL-1R signaling in ground squirrels may have pro-inflammatory implications (Hadj-Moussa et al., 2020). Additional support for NFkB activation during torpor or arousal in WAT and/or BAT from ground squirrels includes the upregulation of genes downstream of NFאB including NLRP3, IL-1 $\beta$, IL-1 $\alpha$, c-Myc, HSP90 $\alpha$, 
SOD1, SOD2, RAGE, IAP, among others (Hadj-Moussa et al., 2020; Logan and Storey, 2021, 2018; Rouble et al., 2014, 2013).

Tissue remodeling proteins had not been investigated in WAT or BAT of a hibernating mammal, to my knowledge. MMPs are important regulators of cytokine release from cells for autocrine, paracrine and endocrine effects, but MMPs can also be regulated by cytokines. For example, MMP2 and MMP3 are upregulated by IL-1 $\beta$ and NFאB signaling but their expression did not correlate with the evidence that $\mathrm{NF} \kappa \mathrm{B}$ is less inhibited during torpor in WAT and BAT, or with data showing that IL-1 $\beta$ is upregulated in BAT during arousal. In terms of tissue-specific differences in tissue remodeling proteins, TIMP2 decreased during torpor, suggesting MMP2 and MMP3 may be less inhibited upon arousal in BAT, but further studies are required to confirm this. MMP2 levels decreased during torpor and early arousal in WAT, but additional the levels of other MMPs and their activities should be assayed to confirm a reduced role for MMPs in WAT during torpor.

The increase in sEGFR in BAT suggested that the activation of cell growth, differentiation and survival pathways mediated by EGFR may be inhibited during early arousal. Ligand binding to EGFR initiates signaling through the Akt-PI3K, Ras-Ras-MEK-ERK, JNK, and p38 pathways, leading to the activation of transcription factors such as STAT5, AP-1, ELK1, and PLC $\gamma$. Therefore, EGFR downregulation upon early arousal may be important to inhibit energy expensive processes until interbout arousal.

Altogether, the data collected in Chapter 3 was not strong enough to conclude that tissue remodeling could or does not occur during torpor or early arousal, given the few changes that were observed. The work done herein was merely a preliminary probe into the possible downstream implications of cytokine signaling and will need to be followed up with more extensive studies. For instance, only one stromelysin and one gelatinase were inspected in terms of their total protein levels, but the levels and activities of collagenases, matrilysins, and 
membrane bound MMPs were not investigated. MMP activity could also be measured in future experiments using in vitro analysis of matrix (e.g. collagen or gelatin) invasion.

\subsubsection{Evaluation of Objective 2}

Quantify markers of tissue inflammation and tissue remodeling during torpor and arousal in ground squirrel adipose tissues.

The objective of Chapter 3 was met. Overall, most markers of inflammation and tissue remodeling were maintained, but IL-1 $\alpha$, sEGFR and TIMP2 were differentially regulated in BAT. In WAT, there was only a significant decrease in MMP2 levels. Together, these results suggested there may be inflammation or tissue remodeling in BAT, and inflammation would be less likely to occur in WAT.

\subsection{Expression profiling of conserved miRNAs}

MicroRNA are small non-coding RNA species that regulate the translation of gene

products by sequestering mRNA from protein synthesis machinery until conditions are favourable for their translation or prohibiting their translation entirely by triggering their degradation. Previously, miRNAs were shown to be differentially regulated in response to torpor in ground squirrels (Liu et al., 2010; Wu et al., 2014) and other hibernators (Luu et al., 2019; Yuan et al., 2015). By measuring the changes in expression of all conserved miRNAs, this thesis expanded on our understanding of the roles of miRNAs in BAT and WAT during torpor. Using high throughput sequencing helped reduce bias in this study since the workflow prevents solely measuring miRNAs associated with specific molecular pathways and because gene expression is not normalized using an arbitrarily chosen reference gene. Instead, read counts were logarithmically transformed and normalized for sequencing depth within samples. This is not to say that RNA-sequencing approaches do not come with their own sources of bias, because they do. For instance, primers in the library creation steps may bind to some miRNA sequences over others, preventing their amplification in the PCR amplification step and detection following 
sequencing. However, sequencing miRNAs using a published, standardized pipeline as was done in the current study can provide a broader, less targeted view of how miRNAs may be regulated in any given tissue to better understand their role in the regulation of metabolic rate suppression.

In Chapters 4 and 5, Illumina NextSeq 500 next-generation sequencing was used to sequence all small RNAs of lengths 150 bp or shorter (including the adapter sequences). Conserved miRNAs (those that had identical sequences to human miRNAs), were identified and analyzed in terms of their expression patterns, enriched KEGG and GO pathways, and possible downstream targets. One important finding from Chapter 4 was that BAT miRNA expression patterns were distinct for control and torpid ground squirrels according to PCA and unsupervised cluster analyses of all miRNAs, and PLS-DA of the 64 miRNAs enriched by random forest feature selection. By contrast, Chapter 5 showed that samples of WAT from control and torpid ground squirrels were not discernable by their miRNA expression patterns. Perhaps if more than seven samples (an $\mathrm{n}=4$ for EC and an $\mathrm{n}=3$ for LT) had been used in small RNA sequencing analysis, this study would have had the statistical power to detect differences in relative miRNA levels between the two conditions. In BAT, more miRNAs (45 out of 76) were upregulated during torpor. In WAT, more miRNAs (20 out of 30) were downregulated during torpor. Finally, no miRNAs were differentially expressed in WAT after correcting for the false discovery rate, suggesting high interindividual variability in miRNA expression and the need for increased biological replicates in future studies. Finally, KEGG and GO terms related to miRNA-mediated inhibition of transcripts were also significantly decreased in WAT of torpid ground squirrels. Altogether, these results suggested a lesser role for miRNAs in the regulation of metabolic rate suppression in WAT compared to BAT.

As expected, miRNAs were implicated in downregulating metabolic pathways in BAT from torpid ground squirrels. KEGG and GO pathway analysis revealed miRNAs were likely to have targets that play roles in transcription, mRNA processing, translation, cell cycle, and energy 
metabolism (glycolysis and oxidative phosphorylation). Ground squirrels pivot from carbohydrate metabolism to fatty acid metabolism during torpor, so the latter result is consistent with increased mRNA levels of perilipin 2 and 4, fatty acid binding protein 3 (FABP3), carnitine/acylcarnitine translocase (SLC25A20), and carnitine palmitoyltransferases CPT1A and CPT2 in BAT, comparing winter ground squirrels to spring ground squirrels (Hampton et al., 2013). Similarly, KEGG and GO pathways associated with transcription, translation, and cell cycle were also predicted to be downregulated by miRNAs in WAT. The overlap in pathways apparently targeted by miRNAs in BAT and WAT is consistent with reports of reduced transcription and translation in hibernators, demonstrated at the transcript, protein and phosphorylation levels of regulation (Hittel and Storey, 2002; Knight et al., 2000; Logan et al., 2019; Tessier et al., 2014).

Unexpectedly, a cluster of myogenic miRNAs (miR-1-3p, miR-206, and miR-133) were differentially expressed in BAT. They could be co-expressed (upregulated by the same transcription factor at the same time, for example) given similar read counts for both miRNAs in each sample. Each of the three miRNAs were downregulated during torpor, but miR-133 may be particularly important considering it is a cold-reduced miRNA whose downregulation is associated with increases in genes involved in adipogenesis and thermogenesis. Similarly, miR206 upregulation is associated with myogenesis and the inhibition of BAT sympathetic activation (Yoon et al., 2018). Thus, downregulation of myomiRs may serve dual roles in preventing brown adipocyte precursors from differentiating into muscle cells and disinhibiting genes associated with thermogenesis, the core function of BAT.

Of considerable interest to me and relevant to the themes of this thesis, KEGG pathways and GO terms relating to cytokine and receptor interactions, the complement cascade, and tissue repair or remodelling were positively enriched in BAT during torpor, meaning genes from these pathways were predicted to be less inhibited by miRNAs. An important observation was that within the larger KEGG pathway of cytokine-cytokine receptor interactions, some families of 
cytokines were less inhibited by miRNAs whereas others were more highly targeted by miRNAs. IL-1-like cytokines were predicted to be less inhibited in BAT. Indeed, IL-1R signaling may be less inhibited during torpor, based on the data from Chapter 2 that showed increased levels of NLRP3 and cleaved gasdermin relative to the full-length protein during torpor, as well as data from Chapter 3 that indicated IL-1 $\alpha$ levels increased without concomitant increases in inhibitors of IL-1 signaling such as IL-1Ra, sIL-1RI or sIL-1RII (Chapter 3). Pathview analysis suggests that IL-1 $\alpha$ may be targeted by miRNAs during torpor, and this may be important to keep IL-1 $\alpha$ levels within a narrow range. However, it is possible that these pathways were predicted to be less targeted by miRNAs during torpor because genes central to these pathways are inhibited at other levels (e.g. DNA methylation, decreased DNA binding capacity or protein levels of the transcription factors that increase their expression, sequestration of transcripts into stress granules). Furthermore, the "less inhibited" pathways may not be supported in BAT. For example, most complementation and coagulation proteins are expressed in liver and not BAT. Therefore, further studies are required to analyze how these "less inhibited" pathways are regulated at the transcriptional, translational, and post-translational levels to determine if they are important in the stress response during torpor.

By contrast, the results of Chapter 5 suggested that miRNAs might inhibit inflammasome activation and IL-1R signaling in WAT, based on NOD-like receptor and TLR receptor signaling pathways being negatively enriched KEGG pathways. Closer inspection of the targeted genes indicated individual inflammasome receptors were not likely targets of miRNAs, but instead, miRNAs were more likely to target caspase-1. This was consistent with the results of Chapter 2, which showed caspase-1 was less active in WAT during torpor and arousal. In addition, miRNAs were predicted to target the kinases that are activated downstream of IL-1 binding to IL-1R (e.g. TAK1, TAB, and IKK). Though caspase-1 activity was markedly decreased during LT and EA, it would be beneficial to investigate cytokine levels from WAT using larger sample sizes to 
determine if there are truly no differences in IL-1 $\alpha$ and IL-1 $\beta$ mRNA and/or protein levels, before discounting a role for IL-1R signaling in the stress response (Chapter 2, Chapter 3). With the current data, WAT miRNA expression patterns appear to be congruent with low inflammasome activation and cytokine signaling in WAT.

Though Chapter 3 showed no change in the protein levels of chemokines MCP1 and MCP3, future studies could explore CXC chemokines and their receptors because this subfamily of cytokines was predicted to be less inhibited in BAT during torpor. Again, it is possible that the sample size was too small to detect real differences in the levels of cytokines in BAT and WAT, because MCP3 appeared to trend towards a 1.5-fold upregulation during EA relative to EC. GO process terms relating to CXCR receptor activation were also highly positively enriched. Some CXC chemokines (e.g. IL-8) are chemotactic for neutrophils while others (e.g. CXCL13) attract adaptive immune cells such as B-cells, prompting their migration into the damaged or infected tissue. Neutrophils are both the most prevalent innate immune cell circulating in the blood of euthermic ground squirrels and the most reduced ones in blood of torpid ground squirrels (Cooper et al., 2016). The levels of circulating platelets, monocytes, eosinophils, and some coagulation factors also decrease during torpor (Cooper et al., 2016). Of note, IL-8, CXCL1, and CXCL5 (CXC chemokines that all activate the same neutrophil receptor, CXCR1) can be upregulated following IL-1 or TNF $\alpha$ signaling and NFאB activation. Thus, increased CXC signaling would be consistent with the results from Chapter 2 and Chapter 3, and a recent study on NFkB signaling (Hadj-Moussa et al., 2020), showing possible IL-1R and NFkB signaling during torpor in BAT. If CXC signaling is increased during torpor (or perhaps in ground squirrels entering torpor), BAT could be a suitable refuge for these cells until arousal. Further exploration into cytokines/chemokines that may be released from BAT and a closer inspection of the cell types that make up BAT at each point of the torpor-arousal cycle may help determine if circulating leukocytes are sequestered in BAT for the length of a torpor-bout. 


\subsubsection{Evaluation of Objective 3 Characterize the conserved adipose microRNAs that change expression in response to torpor.}

The objective of Chapter 4 and 5 was met, since conserved miRNAs were identified, and their expression patterns were characterized for brown and white adipose depots from euthermic control and hibernating squirrels. Overall, 319 miRNAs with conserved sequences were discovered in BAT (45 increased and 31 decreased), and 314 miRNAs were identified in WAT (10 increased and 20 decreased). BAT miRNAs were determined to regulate pathways that are typically downregulated in other organs of metabolically suppressed hibernators including gene expression and carbohydrate metabolism, and WAT miRNAs were predicted to inhibit cell differentiation and growth pathways such as p53 signaling. Important takeaways also include the apparent disinhibition of pathways involved in inflammation and innate immunity which should be further investigated in other ground squirrel tissues as well as other species of hibernator to determine the tissue-specificity and species-specificity of these miRNA profiles.

\subsubsection{Future directions}

The data from all chapters suggest that inflammatory and innate immunity-related pathways may be uninhibited at certain points of the torpor-arousal cycle, in both WAT and BAT. This is an important finding that will hopefully prompt the study of innate immune pathways, especially the regulation of inflammasomes that are capable of sensing and responding to endogenous damage markers, in other tissues of hibernating ground squirrels as well as other species capable of metabolic rate suppression. For instance, mature, conserved miRNAs were differentially expressed in a manner that could enable NOD-like signaling or IL-1 signaling in BAT but based on the expression profiles of the 314 identified miRNAs, probably not in WAT. Thus, there may be tissue-specific differences in the regulation of NLRs and ALRs in other hibernator tissues. However, not all miRNAs were investigated in Chapter 4 and 5. Firstly, the method of 
library preparation including RNA extraction, adapter ligation, amplification, and purification from the PAGE gel, may enrich some miRNA sequences over others. Novel miRNAs, or miRNAs that differ by at least one nucleotide compared to the human sequence, were not characterized as part of these projects. This remains an important avenue for exploration, since other studies have distinguished novel mature miRNAs that could be associated with hibernation. In a study comparing the small RNA-sequencing libraries of brain and WAT from $n=1$ control and torpid bats, Myotis ricketti, 49 miRNAs were discovered to be differentially regulated (Yuan et al., 2015). Of these, 31 were bat-specific, "novel" miRNAs. Therefore, it is expected that ground squirrel BAT and WAT will also differentially express ground squirrel-specific novel miRNAs during torpor.

The studies herein also suggested that NFkB may play an important role in the priming of the NLRP3 inflammasome in BAT upon arousal (Chapter 2), and cytokine signaling leading to the activation of kinases controlling NFkB activity may also be increased during torpor and arousal (Chapter 3). However, there have been no experiments exploring the regulation of NFkB at more than two time point of the torpor-arousal cycle in ground squirrel BAT. Only a single study has been done profiling the activation of $\mathrm{NF \kappa B}$ in WAT and BAT from control and hibernating ground squirrels. This study was limited in that it did not investigate the proteolysis of precursor proteins p105 and p100 into their active forms, p50 and p52, respectively. It also did not investigate the subcellular localization of NFkB dimers, or the DNA binding capacity of NFkB found in the nuclear protein fraction. Additionally, the phosphorylation status of $\operatorname{IKK} \alpha / \beta$, the kinase that

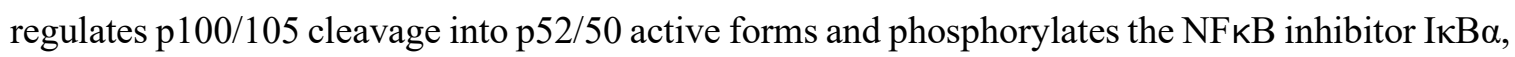
was not measured. The studies performed in this thesis, as well as others, suggest that NFkB could be activated during torpor and arousal in hibernator BAT, based on the upregulation of several genes known to be regulated by NFkB. For instance, c-Myc and RAGE are upregulated in both WAT and BAT during torpor (Hadj-Moussa et al., 2020; Logan and Storey, 2018). In WAT, other NFkB-regulated genes are upregulated during torpor including X-IAP, SOD1 and SOD2. In 
aroused ground squirrel BAT, there were increases in the transcript levels of NLRP3, IL-1 $\beta$, and IL- $1 \alpha$ and the protein levels of HSP90 $\alpha$ and RAGE. Given that NFKB can regulate a range of processes including inflammation, autophagy, immunity (e.g. cell adhesion, tissue remodeling, chemokine production), and cell survival, it may be worthwhile conducting a study where chromatin immunoprecipitation is used to identify which genes (and therefore which processes) may be regulated by NFKB in the WAT and BAT sampled from torpid and aroused ground squirrels.

Just a few biomarkers for extracellular matrix reorganization were measured in Chapter 3 of this thesis. Future studies could elaborate on this work by exploring the makeup of the extracellular matrix using immunohistochemistry or cell staining for extracellular components (e.g. trichrome staining for collagen) could provide valuable information regarding ECM plasticity and the specific make-up of the extracellular membrane at different points of the torpor-arousal cycle. It is postulated that PUFAs may be important in the maintenance of cell membrane fluidity during torpor, to prevent cell rigidity, but assays such as the parallel artificial membrane permeability assay could be done to measure relative cell permeabilization. Finally, metabolomic and proteomic analyses of the lipids, sugars, and proteins that make up the cell membrane, the extracellular matrix, and the contents of white and brown adipocytes would be worthwhile doing to determine if PUFAs that are consumed during the fall hyperphagic period are used in the cell membrane or as fuel for lipid oxidation. This is especially important to do because the products of omega- 6 fatty acids are typically proinflammatory. For instance, arachidonic acid is used to synthesize prostaglandins, pyretic and pro-inflammatory molecules that have yet to be investigated in the WAT and BAT of hibernating ground squirrels. By contrast, docosahexaenoic acid and eicosapentaenoic acid have largely anti-inflammatory properties in mammals. Thus, metabolomic analysis could be useful to more deeply explore how inflammation could be regulated over a torpor bout in WAT and BAT. 
Herein, each study was performed on male ground squirrels only, but it would be interesting to compare male and female ground squirrels (using a two-way ANOVA) in terms of inflammasome protein levels, relative cytokine and extracellular matrix reorganization biomarker abundances, and differential miRNA levels. Similarly, using captive ground squirrels whose ages are known may help provide insight into how these inflammatory and stress-responsive pathways are regulated as these relatively long-lived animals age. In addition, knowing the age of the adult ground squirrels may help reduce variability in the relative transcript and protein levels since life history would be controlled for. Instead, the data presented herein aim to provide insight into the general stress response of wild, adult, male ground squirrels, where significant changes are anticipated to scale for the larger wild population of ground squirrels.

Finally, identifying WAT and BAT cell types and their distribution during torpor/early arousal would help clarify whether adipose tissues serve as depots for immune cells during torpor, or if cell differentiation/maturation is necessary to maintain tissue homeostasis. Microscopy, immunohistochemistry, or flow cytometry experiments for the identification of cell types and their localization in adipose tissues would strengthen our understanding of both innate immunity and tissue remodeling processes in hibernating mammals. Regarding cell differentiation, there are still many questions to answer about the capacity of hibernator white adipocytes to initiate beige-ing in the pre-hibernation period or during torpor. However, the apparent miRNA-mediated downregulation of MDM2, an inhibitor of p53, an important transcription factor that promotes cell senescence and DNA repair pathways, suggests tissues like WAT likely prevent cell differentiation during torpor.

\subsubsection{Concluding remarks}

The core idea linking the chapters of this thesis is that adipose tissues likely use protective mechanisms to sense natural endogenous stress and respond to it while in torpor. In BAT, data from Chapter 2-4 suggest that inflammasomes, highly regulated components of the 
innate immune system that respond to endogenous and pathogenic stimuli, may indeed be activated during torpor and arousal. Supporting a role for IL-1R signaling downstream of inflammasome activation, IL-1 $\alpha$ and IL-1 $\beta$ were upregulated during torpor and arousal, respectively. MicroRNAs were shown to have diverse roles in the inhibition of processes that have been shown to be downregulated during torpor, including transcription, translation, cell cycle, glycolysis and gluconeogenesis. BAT miRNAs likely do not target NOD-like receptor signaling or cytokine/cytokine receptor pathways, including IL-1R signaling, suggesting that a coordinated upregulation of innate immune pathways could be possible in this tissue. By contrast, miRNAs in WAT may target caspase-1 and the kinases that activate pro-inflammatory signaling cascades, which was shown to be consistent with decreases in caspase-1 activity and very few changes in the expression of cytokines and tissue remodeling proteins during late torpor and early arousal. The studies herein show for the first time that inflammasome signaling, inflammatory cytokines and tissue remodeling proteins, and miRNA profiles are differentially regulated in the WAT and BAT of hibernating ground squirrels. These studies provide support for the idea that certain hibernator tissues may upregulate inflammatory signaling during, or following arousal from, a state of metabolic rate suppression. 


\subsection{References}

Cogut, V., Bruintjes, J.J.J., Eggen, B.J.L.J.L., van der Zee, E.A.A., Henning, R.H.H., 2017. Brain inflammatory cytokines and microglia morphology changes throughout hibernation phases in Syrian hamster. Brain. Behav. Immun. 68, 17-22. https://doi.org/10.1016/j.bbi.2017.10.009

Cooper, S., Sell, S., Nelson, L., Hawes, J., Benrud, J.A., Kohlnhofer, B.M., Burmeister, B.R., Flood, V.H., 2016. Von Willebrand factor is reversibly decreased during torpor in 13-lined ground squirrels. J. Comp. Physiol. B Biochem. Syst. Environ. Physiol. 186, 131-139. https://doi.org/10.1007/s00360015-0941-5

Hadj-Moussa, H., Wijenayake, S., Storey, K.B., 2020. Multi-tissue profile of NFkB pathway regulation during mammalian hibernation. Comp. Biochem. Physiol. Part - B Biochem. Mol. Biol. 246-247. https://doi.org/10.1016/j.cbpb.2020.110460

Hampton, M., Melvin, R.G., Andrews, M.T., 2013. Transcriptomic analysis of brown adipose tissue across the physiological extremes of natural hibernation. PLoS One 8, 1-12. https://doi.org/10.1371/journal.pone.0085157

Hittel, D., Storey, K.B., 2002. The translation state of differentially expressed mRNAs in the hibernating 13-lined ground squirrel (Spermophilus tridecemlineatus). Arch. Biochem. Biophys. 401, 244-254. https://doi.org/10.1016/S0003-9861(02)00048-6

Knight, J.E., Narus, E.N., Martin, S.L., Jacobson, A., Barnes, B.M., Boyer, B.B., 2000. mRNA stability and polysome loss in hibernating Arctic ground squirrels (Spermophilus parryii). Mol. Cell. Biol. 20, 6374-6379. https://doi.org/10.1128/MCB.20.17.6374-6379.2000

Kurtz, C.C., Lindell, S.L., Mangino, M.J., Carey, H. V, 2006. Hibernation confers resistance to intestinal ischemia-reperfusion injury. Am. J. Physiol. Liver Physiol. 291, G895-G901. https://doi.org/10.1152/ajpgi.00155.2006

Liu, Y., Hu, W., Wang, H., Lu, M., Shao, C., Menzel, C., Yan, Z., Li, Y., Zhao, S., Khaitovich, P., Liu, M., Chen, W., Barnes, B.M., Yan, J., 2010. Genomic analysis of miRNAs in an extreme mammalian hibernator, the Arctic ground squirrel. Physiol. Genomics 42 A, 39-51. https://doi.org/10.1152/physiolgenomics.00054.2010

Logan, S.M., Storey, K.B., 2021. Inflammasome signaling could be used to sense and respond to endogenous damage in brown but not white adipose tissue of a hibernating ground squirrel. Dev. Comp. Immunol. 114, 103819. https://doi.org/10.1016/j.dci.2020.103819

Logan, S.M., Storey, K.B., 2018. Pro-inflammatory AGE-RAGE signaling is activated during arousal from hibernation in ground squirrel adipose. PeerJ 6, e4911. https://doi.org/10.7717/peerj.4911

Logan, S.M., Wu, C., Storey, K.B., 2019. The squirrel with the lagging eIF2: Global suppression of protein synthesis during torpor. Comp. Biochem. Physiol. Part A Mol. Integr. Physiol. 227, 161-171. https://doi.org/10.1016/j.cbpa.2018.10.014

Luu, B.E., Lefai, E., Giroud, S., Swenson, J.E., Chazarin, B., Gauquelin-Koch, G., Arnemo, J.M., Evans, A.L., Bertile, F., Storey, K.B., 2019. MicroRNAs facilitate skeletal muscle maintenance and metabolic suppression in hibernating brown bears. J. Cell. Physiol. jcp.29294. https://doi.org/10.1002/jcp.29294

MacCannell, A., Sinclair, K., Friesen-Waldner, L., McKenzie, C.A., Staples, J.F., 2017. Water-fat MRI in a hibernator reveals seasonal growth of white and brown adipose tissue without cold exposure. J. Comp. Physiol. B Biochem. Syst. Environ. Physiol. 187, 759-767. https://doi.org/10.1007/s00360017-1075-8

Musacchia, X.J., Barr, R.E., 1968. Survival of whole-body-irradiated hibernating and active ground squirrels; Citellus tridecemlineatus. Radiat. Res. 33, 348. https://doi.org/10.2307/3572485

Orr, A.L., Lohse, L.A., Drew, K.L., Hermes-Lima, M., 2009. Physiological oxidative stress after arousal 
from hibernation in Arctic ground squirrel. Comp. Biochem. Physiol. 153, 213-221. https://doi.org/10.1016/j.cbpa.2009.02.016.Physiological

Rouble, A.N., Hefler, J., Mamady, H., Storey, K.B., Tessier, S.N., 2013. Anti-apoptotic signaling as a cytoprotective mechanism in mammalian hibernation. PeerJ 1, e29. https://doi.org/10.7717/peerj.29

Rouble, A.N., Tessier, S.N., Storey, K.B., 2014. Characterization of adipocyte stress response pathways during hibernation in 13-lined ground squirrels. Mol. Cell. Biochem. 393, 271-282. https://doi.org/10.1007/s11010-014-2070-y

Storey, K.B., 2010. Out cold: biochemical regulation of mammalian hibernation - A mini-review. Gerontology 56, 220-230. https://doi.org/10.1159/000228829

Tessier, S.N., Audas, T.E., Wu, C.W., Lee, S., Storey, K.B., 2014. The involvement of mRNA processing factors TIA-1, TIAR, and PABP-1 during mammalian hibernation. Cell Stress Chaperones 1-13. https://doi.org/10.1007/s12192-014-0505-8

Wu, C.-W., Biggar, K.K., Storey, K.B., 2014. Expression profiling and structural characterization of microRNAs in adipose tissues of hibernating ground squirrels. Genomics. Proteomics Bioinformatics 12, 284-291. https://doi.org/10.1016/j.gpb.2014.08.003

Yoon, Y.-S., Tsai, W.-W., Van de Velde, S., Chen, Z., Lee, K.-F., Morgan, D.A., Rahmouni, K., Matsumura, S., Wiater, E., Song, Y., Montminy, M., 2018. cAMP-inducible coactivator CRTC3 attenuates brown adipose tissue thermogenesis. Proc. Natl. Acad. Sci. 115, E5289-E5297. https://doi.org/10.1073/pnas.1805257115

Yuan, L., Geiser, F., Lin, B., Sun, H., Chen, J., Zhang, S., 2015. Down but not out: the role of microRNAs in hibernating bats. PLoS One 10, e0135064. https://doi.org/10.1371/journal.pone.0135064 


\section{Appendices}

\section{A. Representative immunoblots \\ NALP1}
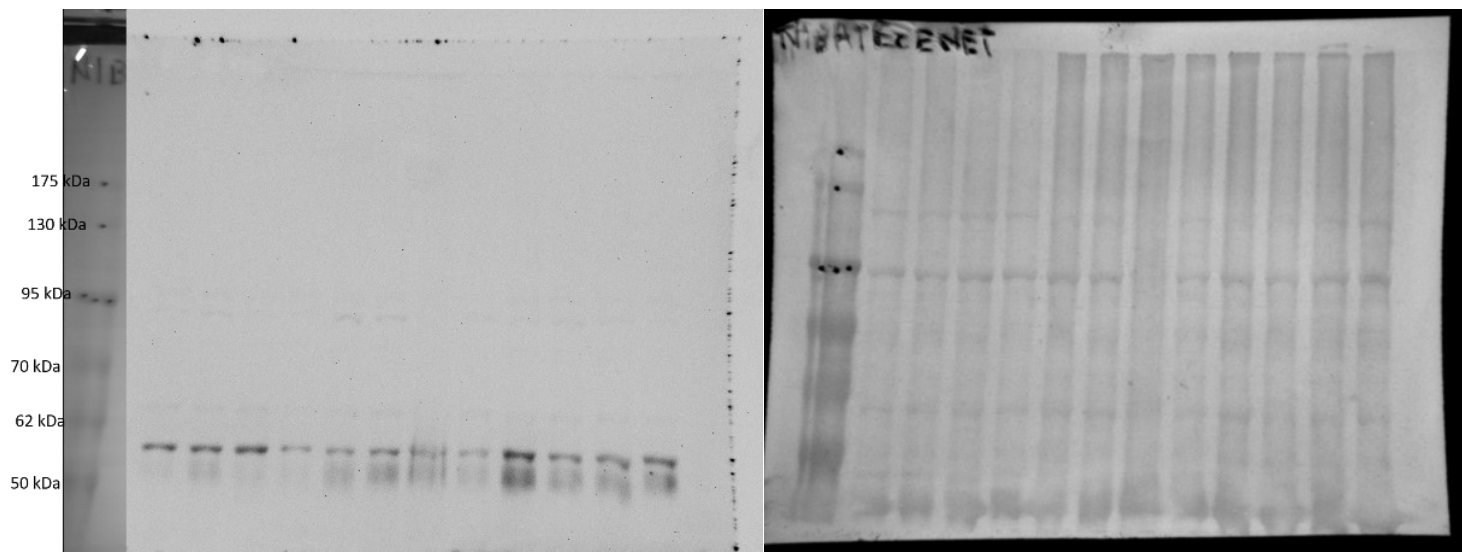

Figure A.6.1: 13-lined ground squirrel BAT NLRP1 western blot showing bands for three time points (EC, EN and ET) using an $\mathrm{n}=4$ biological replicates, and the corresponding Coomassie stained membrane. A range of several bands on the Coomassie stained membrane were used to control for protein loading

\section{NLRP3}

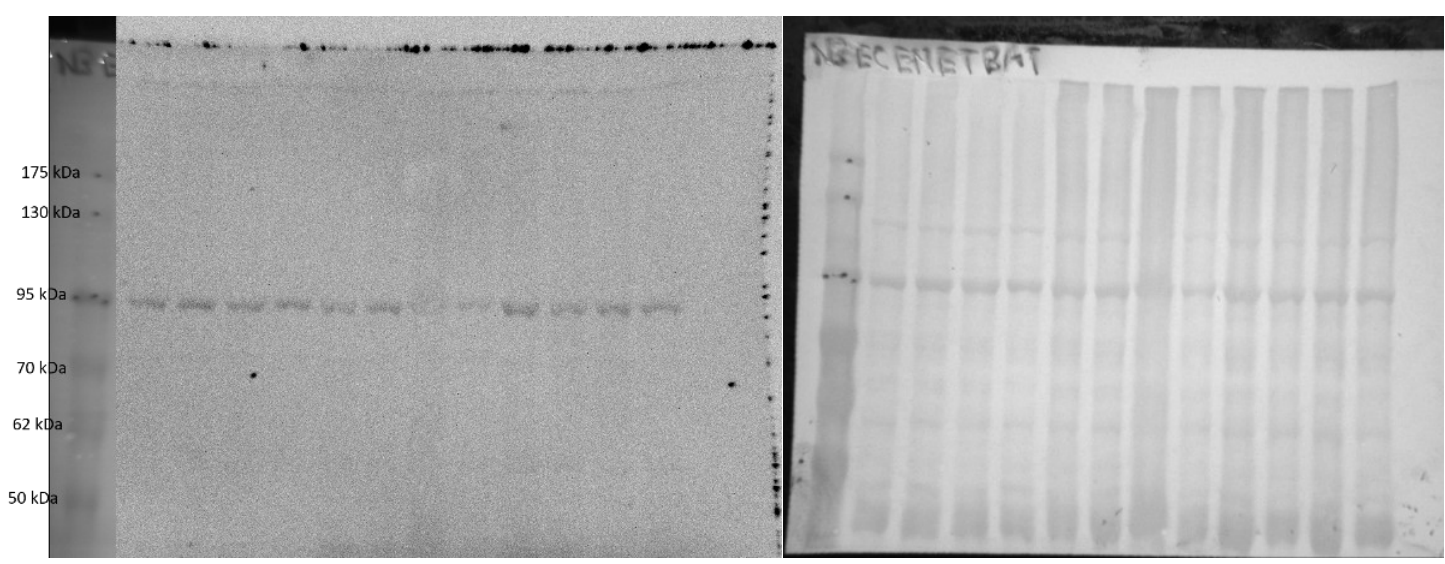

Figure A.6.2: 13-lined ground squirrel BAT NLRP3 western blot showing bands for three time points (EC, EN and ET) using an $\mathrm{n}=4$ biological replicates and the corresponding Coomassie stained membrane

AIM2 


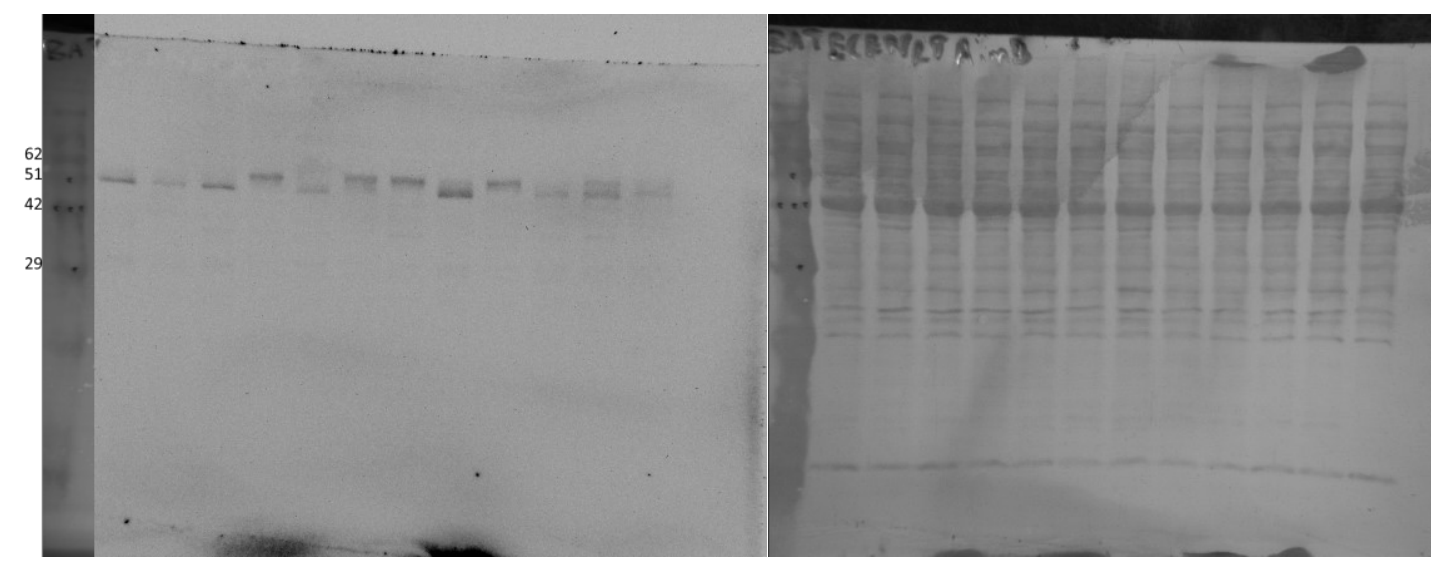

Figure A.6.3: 13-lined ground squirrel BAT AIM2 western blot showing bands for three time points (EC, EN and LT) using an $n=4$ biological replicates and the corresponding Coomassie stained membrane

\section{Caspase-1}

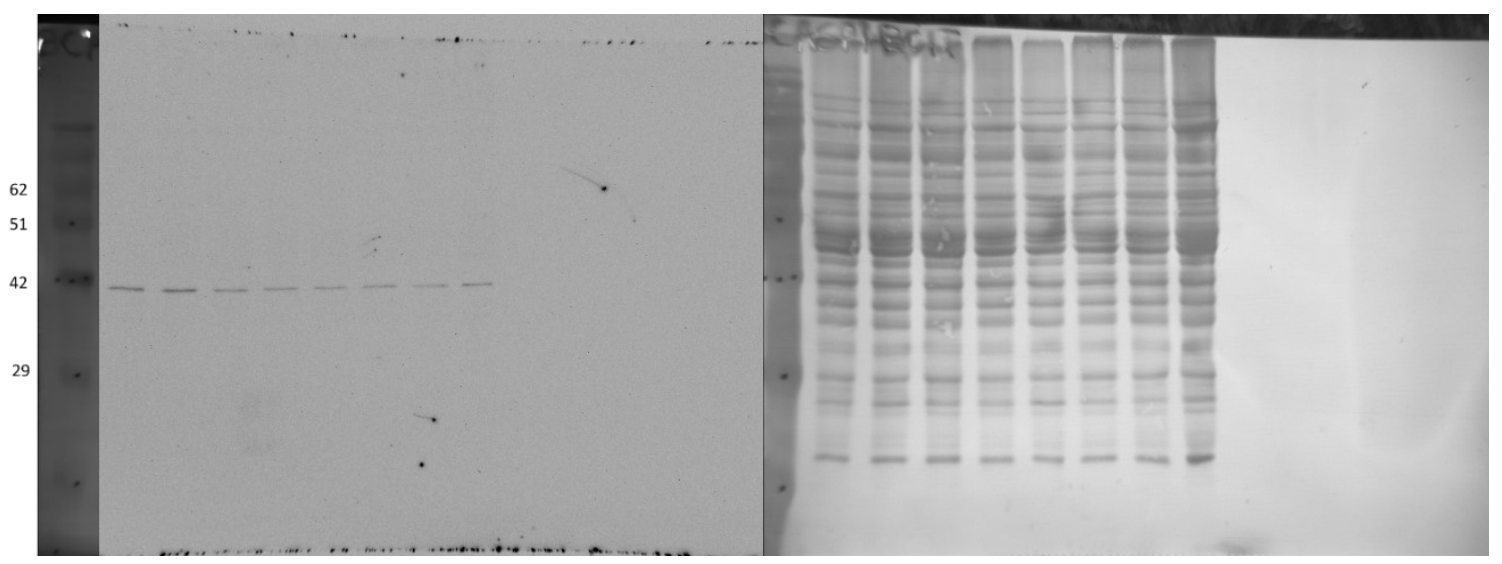

Figure A.6.4: 13-lined ground squirrel BAT Caspase-1 western blot and Coomassie stained membrane

\section{Caspase-5}

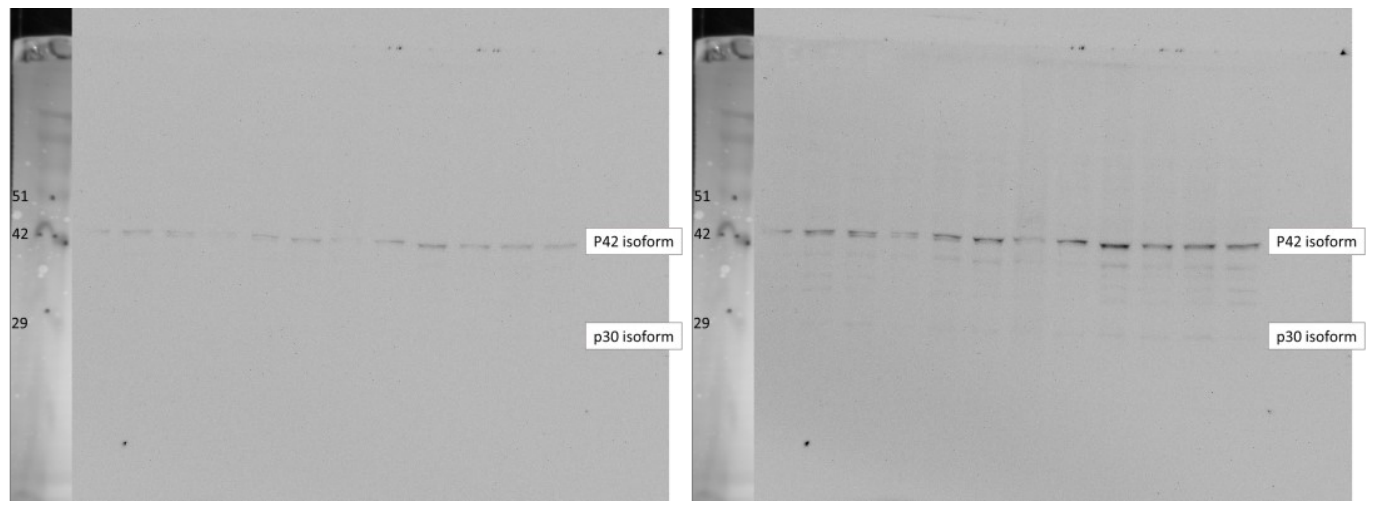


Figure A.6.5: 13-lined ground squirrel BAT Caspase-5 western blot at short and long exposure times. Both western blots show $\mathrm{n}=4$ biological replicates for three time points of the torpor-arousal cycle (EC, EN and LT). Bands appearing at correct molecular weight were quantified as p42 and p30 isoforms.

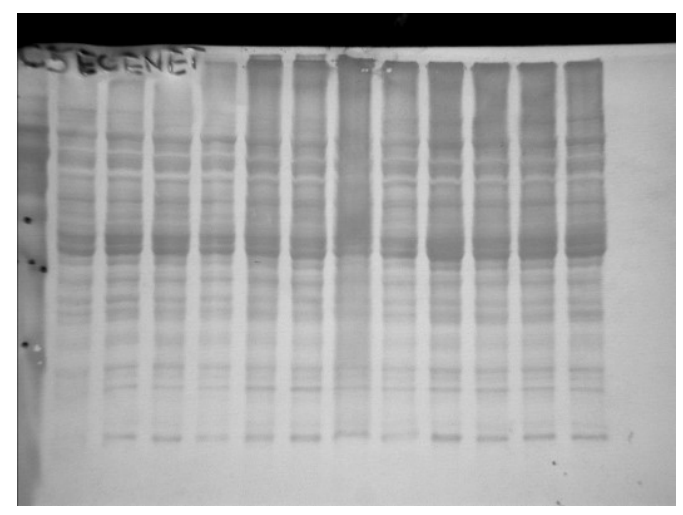

Figure A.6.6: The Coomassie blue stained PVDF membrane corresponding to the caspase-5-probed western blot, showing $n=4$ biological replicates for 3 time points of the torpor-arousal cycle, EC, EN and ET.

\section{Gasdermin D}

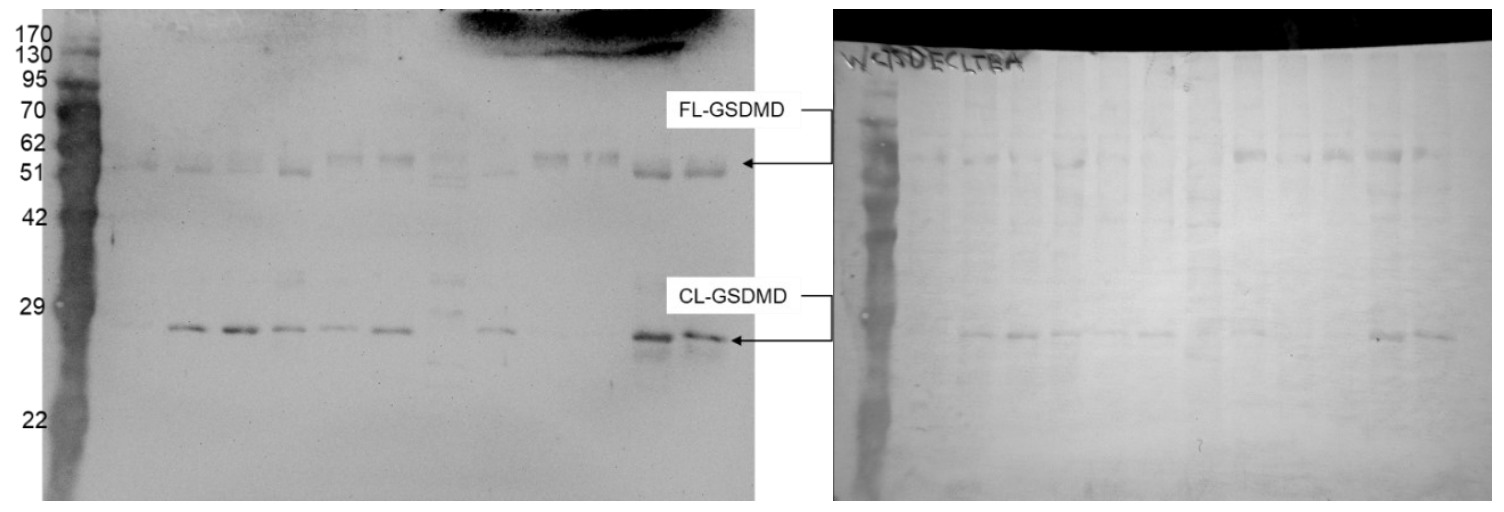

Figure A.6.7: 13-lined ground squirrel WAT western blot showing full-length (FL) and cleaved (CL) gasdermin $\mathrm{D}$ as the only two bands on the blot, and the corresponding Coomassie stained membrane showing low protein abundance in this tissue. Three time points are shown (EC, LT and EA) using an $\mathrm{n}=4$ biological replicates.

\section{Interleukin 1 Beta}




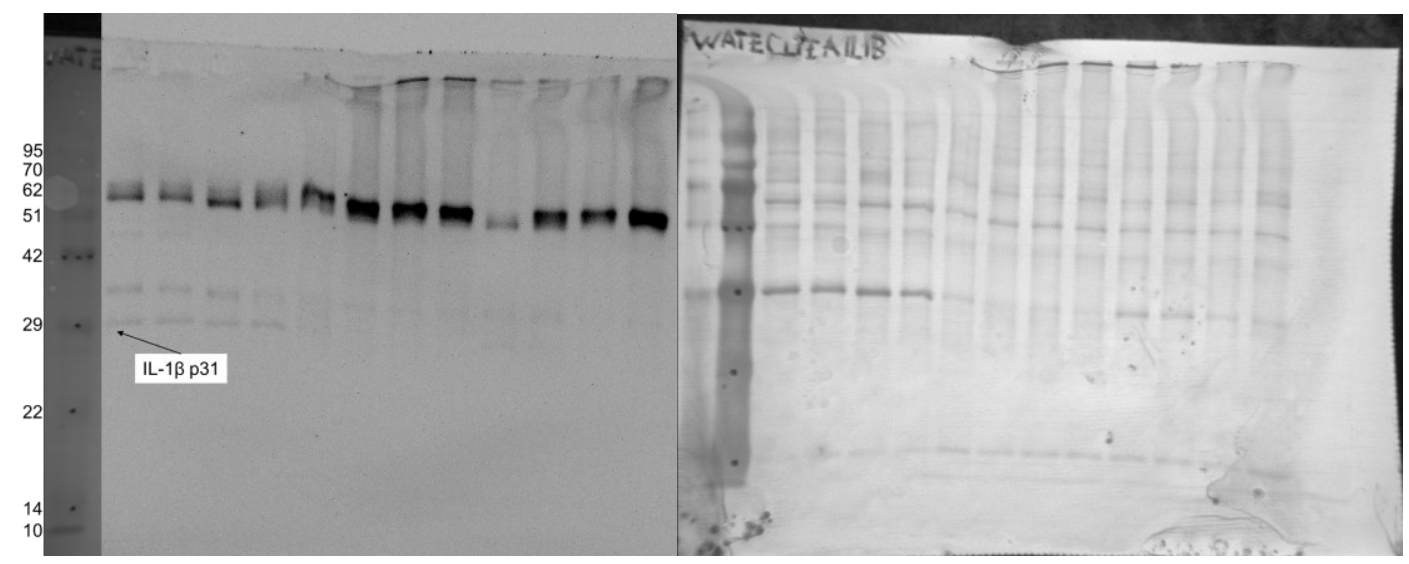

Figure A.6.8: 13-lined ground squirrel WAT IL-1 beta western blot showing bands for three time points (EC, LT and EA) using an $n=4$ biological replicates, and the corresponding Coomassie stained membrane.

\section{Interleukin 18}

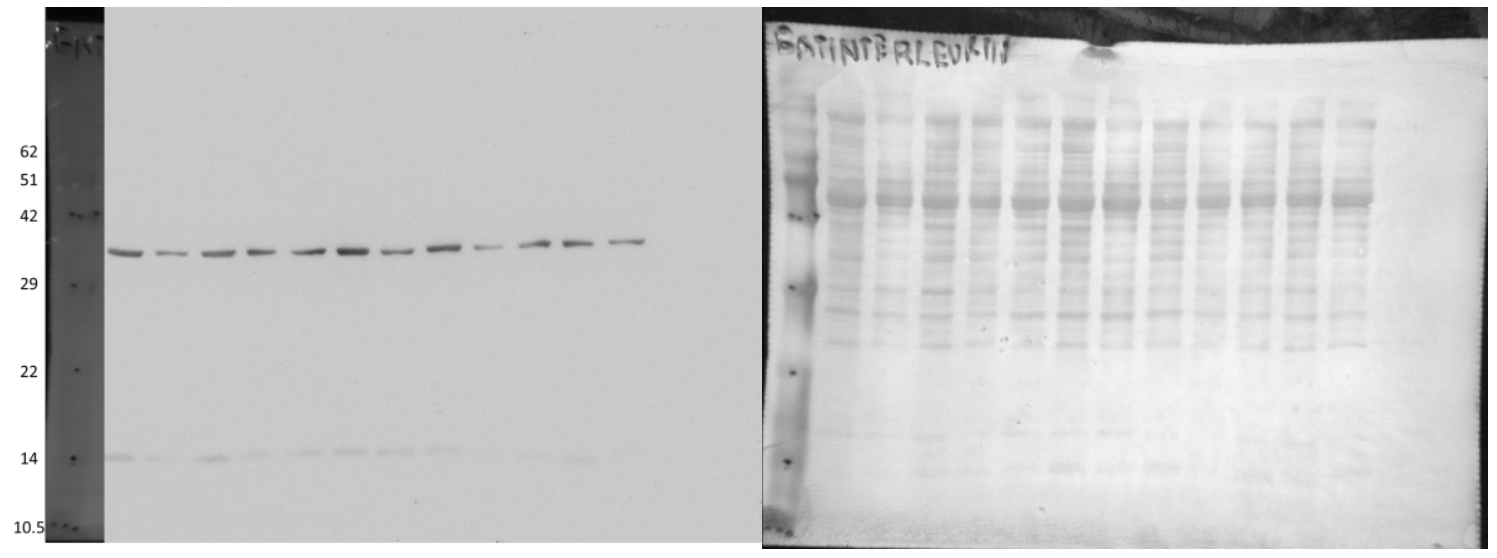

Figure A.6.9: 13-lined ground squirrel WAT IL-18 western blot showing bands for three time points (EN, ET and LT) using an $n=4$ biological replicates, and the corresponding Coomassie stained membrane.

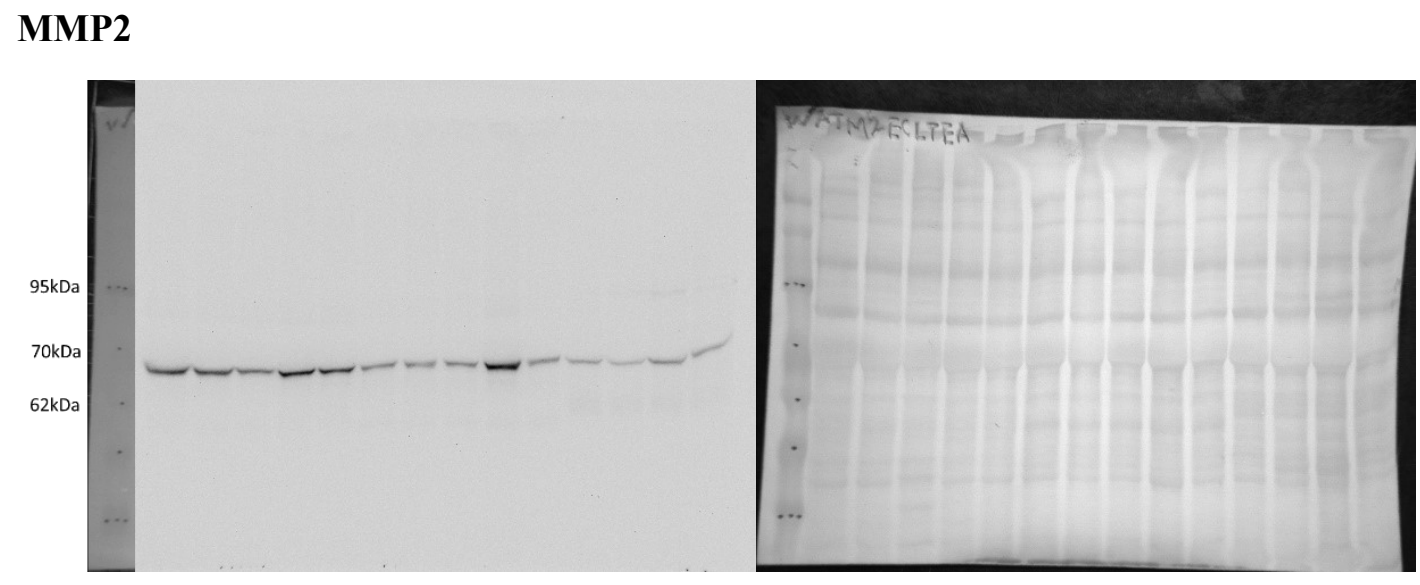


Figure A.10: 13-lined ground squirrel WAT MMP2 western blot showing bands for three time points (EC, LT and EA) using an $n=4$ biological replicates, and the corresponding Coomassie stained membrane.

\section{MMP3}

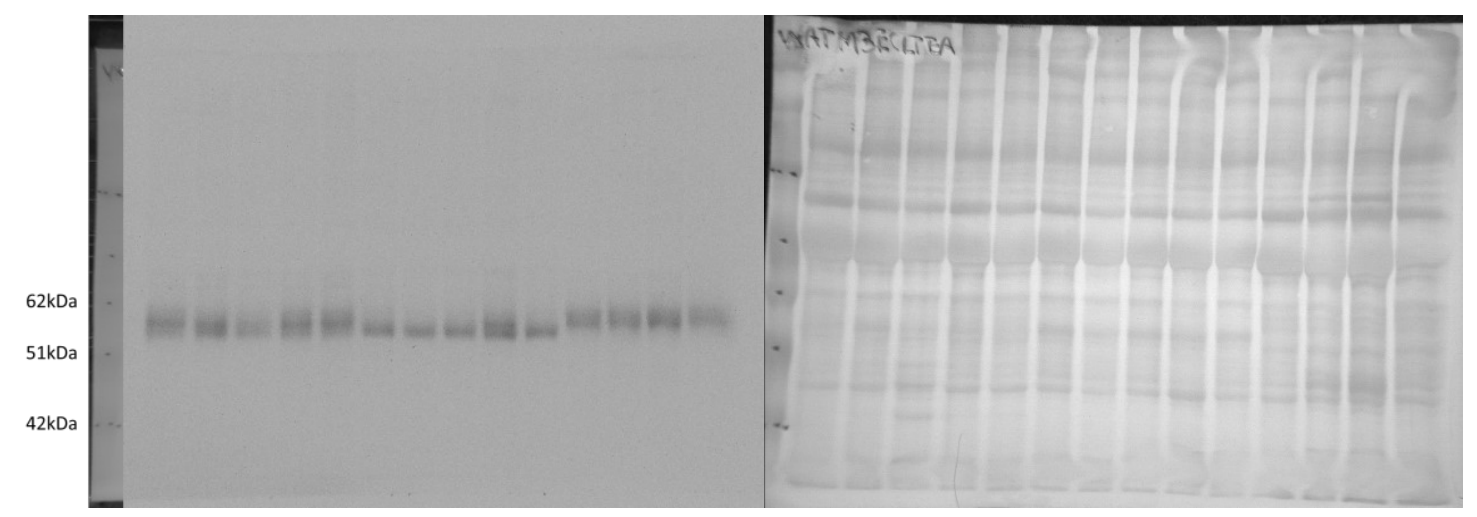

Figure A.11: 13-lined ground squirrel WAT MMP3 western blot showing bands for three time points (EC, LT and EA) using an $n=5$ biological replicates for EC and LT and $n=4$ biological replicates for EA, and the corresponding Coomassie stained membrane.

\section{P53}

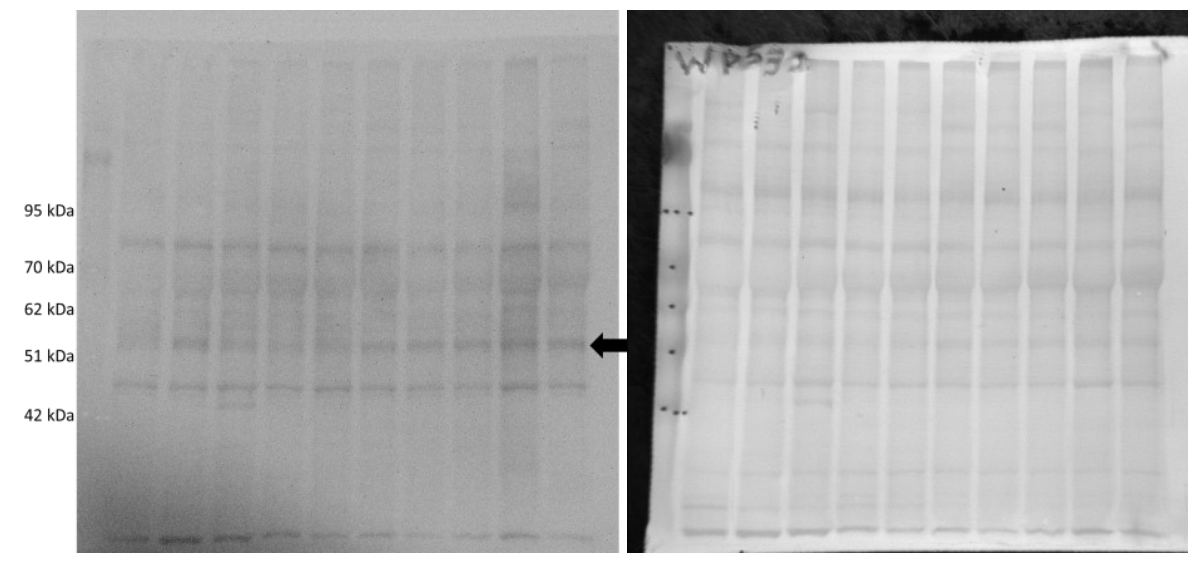

Figure A.12: 13-lined ground squirrel WAT total p53 western blot showing bands for two time points (EC and LT) using an $\mathrm{n}=5$ biological replicates, and the corresponding Coomassie stained membrane. Band at the correct molecular weight was quantified. 


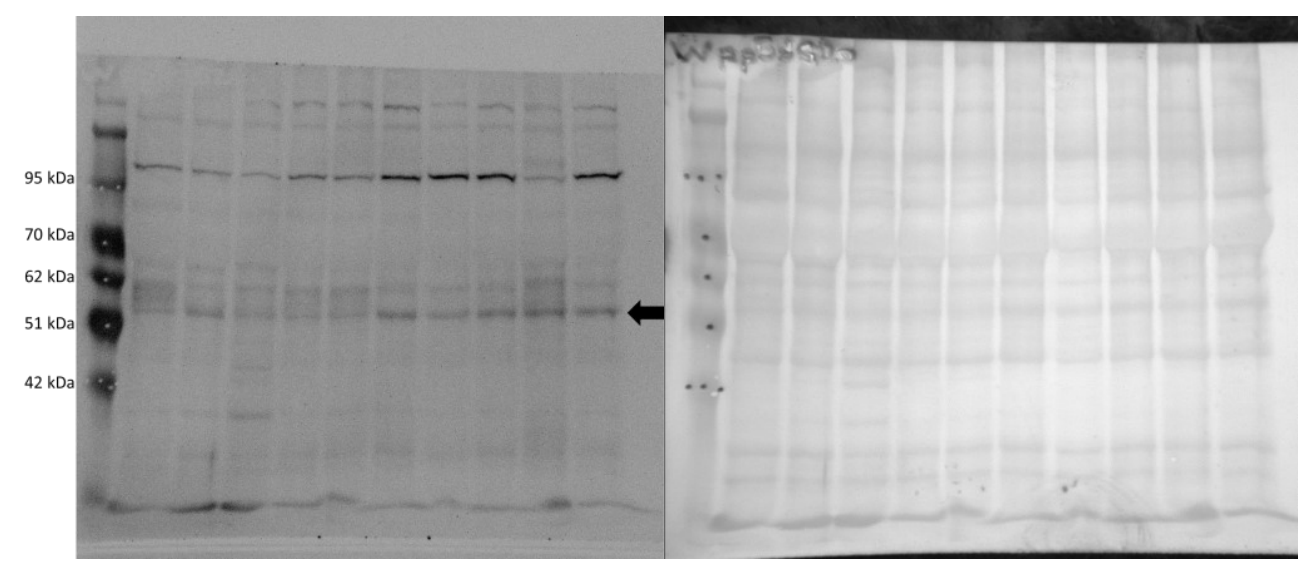

Figure A.13: 13-lined ground squirrel WAT phospho-p53 (S20) western blot showing bands for two time points (EC and LT) using an $n=5$ biological replicates, and the corresponding Coomassie stained membrane. Band at the correct molecular weight was quantified.

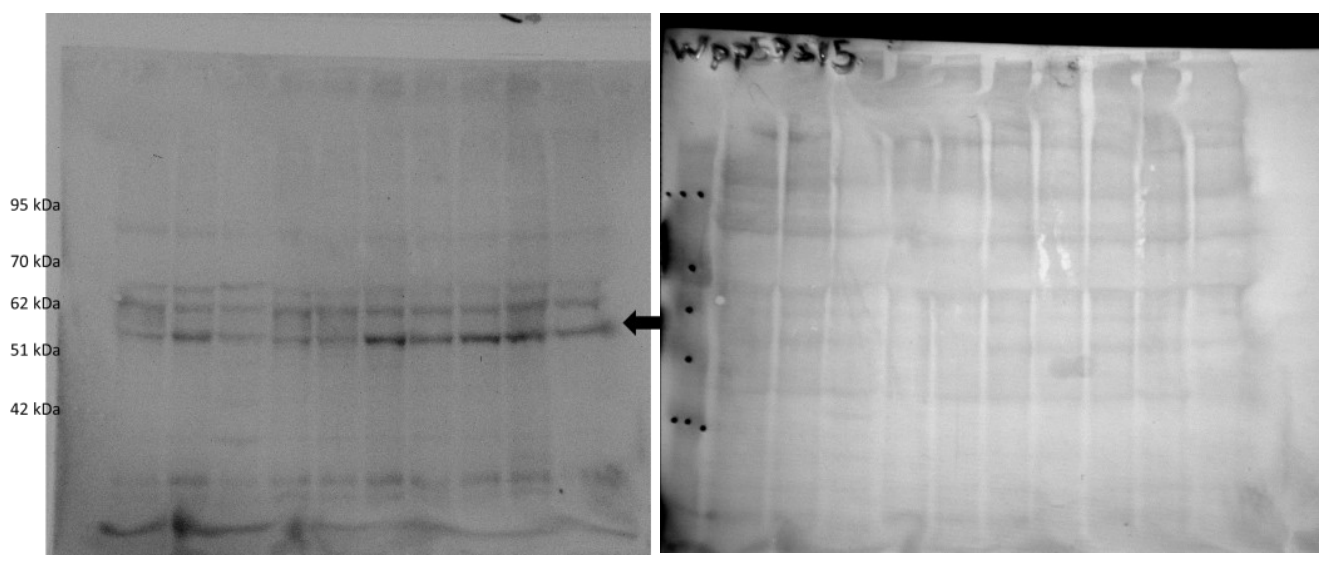

Figure A.14: 13-lined ground squirrel WAT phospho-p53 (S15) western blot showing bands for two time points (EC and LT) using an $\mathrm{n}=5$ biological replicates, and the corresponding Coomassie stained membrane. Band at the correct molecular weight was quantified. 


\section{B. Bioinformatic check of antibody compatibility}

When the epitope was provided by the company, it was possible to do a protein alignment using NCBI-derived amino acid sequences and Clustal Omega. The following is an example of what was done to determine if an antibody should be able to bind the ground squirrel protein:

First, a multi-species alignment was done including Ictidomys tridecemlineatus (13LGS) and the species whose protein/peptide was used as the antigen.

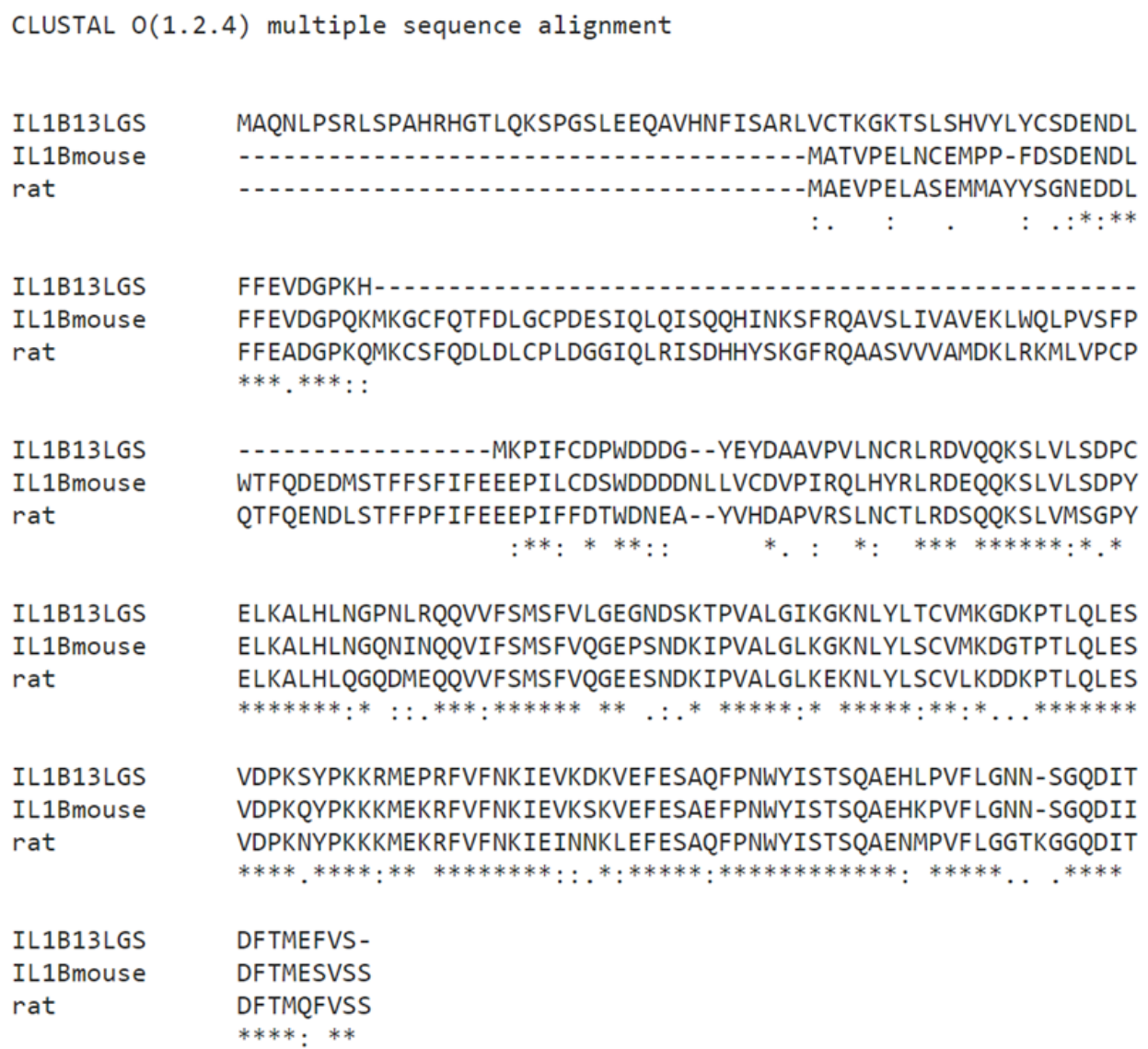

Figure B.1: Alignment of IL-1 beta from ground squirrel, mouse (Mus musculus) and rat (Rattus norvegicus) to get a view of overall sequence similarity.

If the epitope was available, the epitope was aligned with the ground squirrel sequence to determine sequence similarity. 


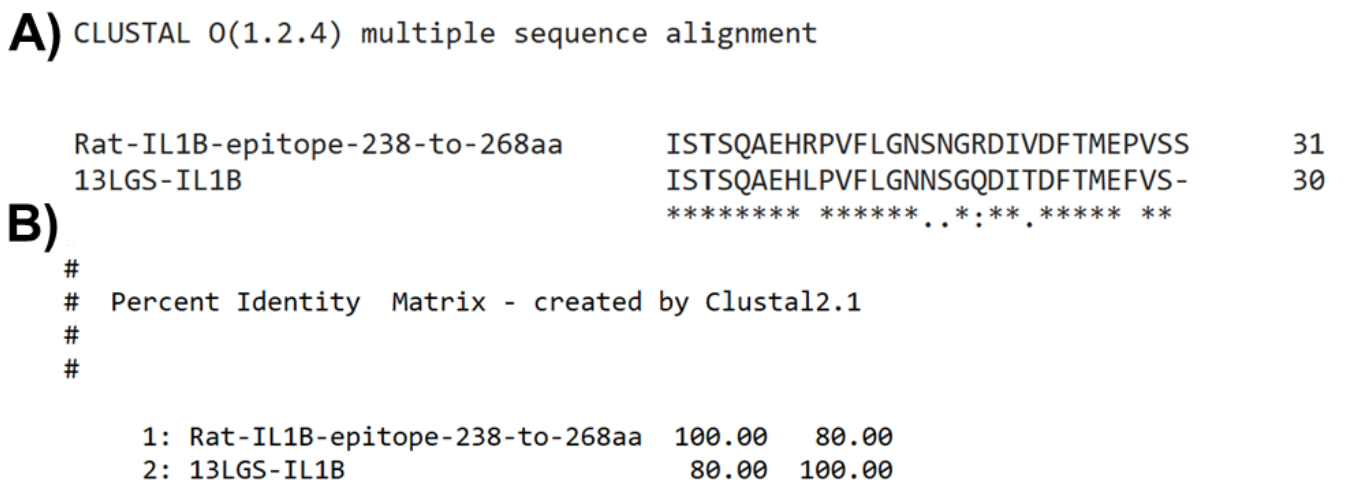

Figure B.2: Alignment of 13-lined ground squirrel amino acid sequence corresponding to the epitope for the Santa Cruz Biotech. Antibody against IL-1 beta (\#sc-515598)

The epitopes that the antibodies used in the multiplex assays are proprietary so we cannot determine how conserved the epitope is between ground squirrels and related mammals. However, we can align the predicted ground squirrel sequence with the amino acid sequence of humans to help assess if the antibody should be able to bind the ground squirrel protein. For example, ground squirrel TIMP3 is $99.1 \%$ similar to human TIMP3:

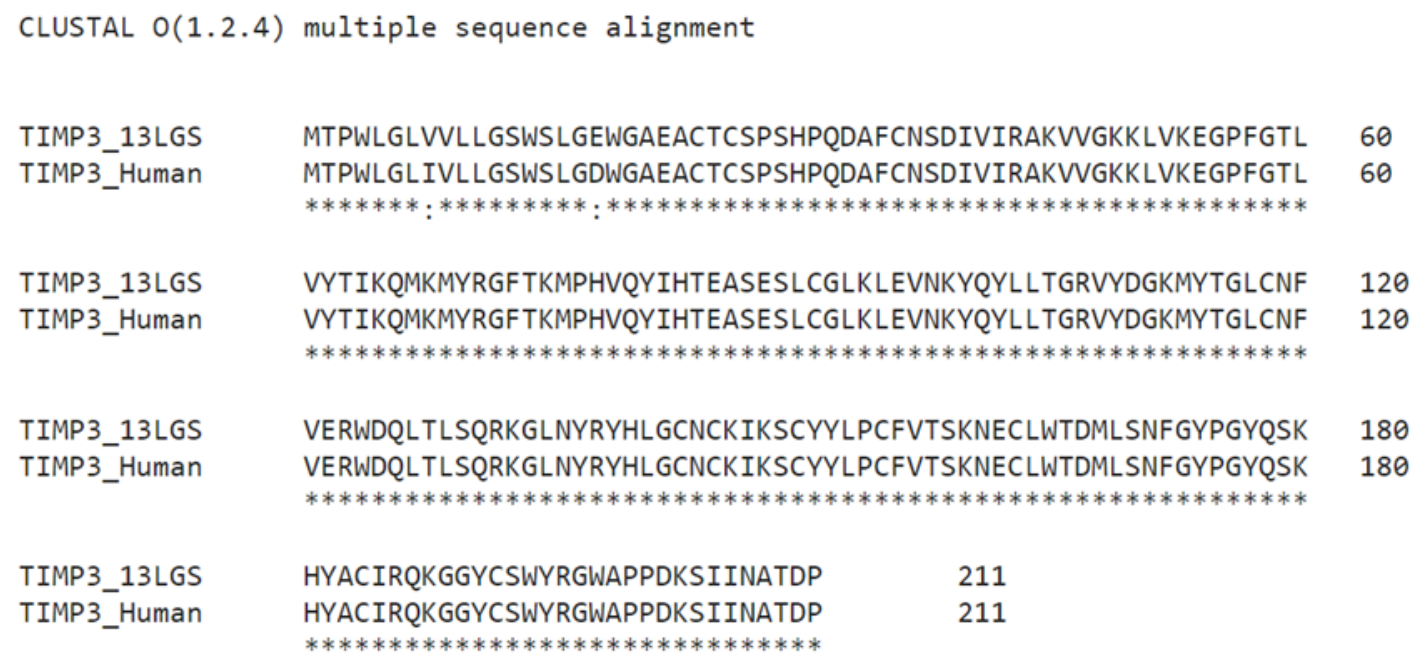

60 60

Figure B.3: Alignment of TIMP3 protein sequences from human (NP_000353.1) and ground squirrel (XP_005322373.1). 


\section{Caspase-1 activity assay}

Standard curve

The absorbance values were blank subtracted before plotting the standard curve.

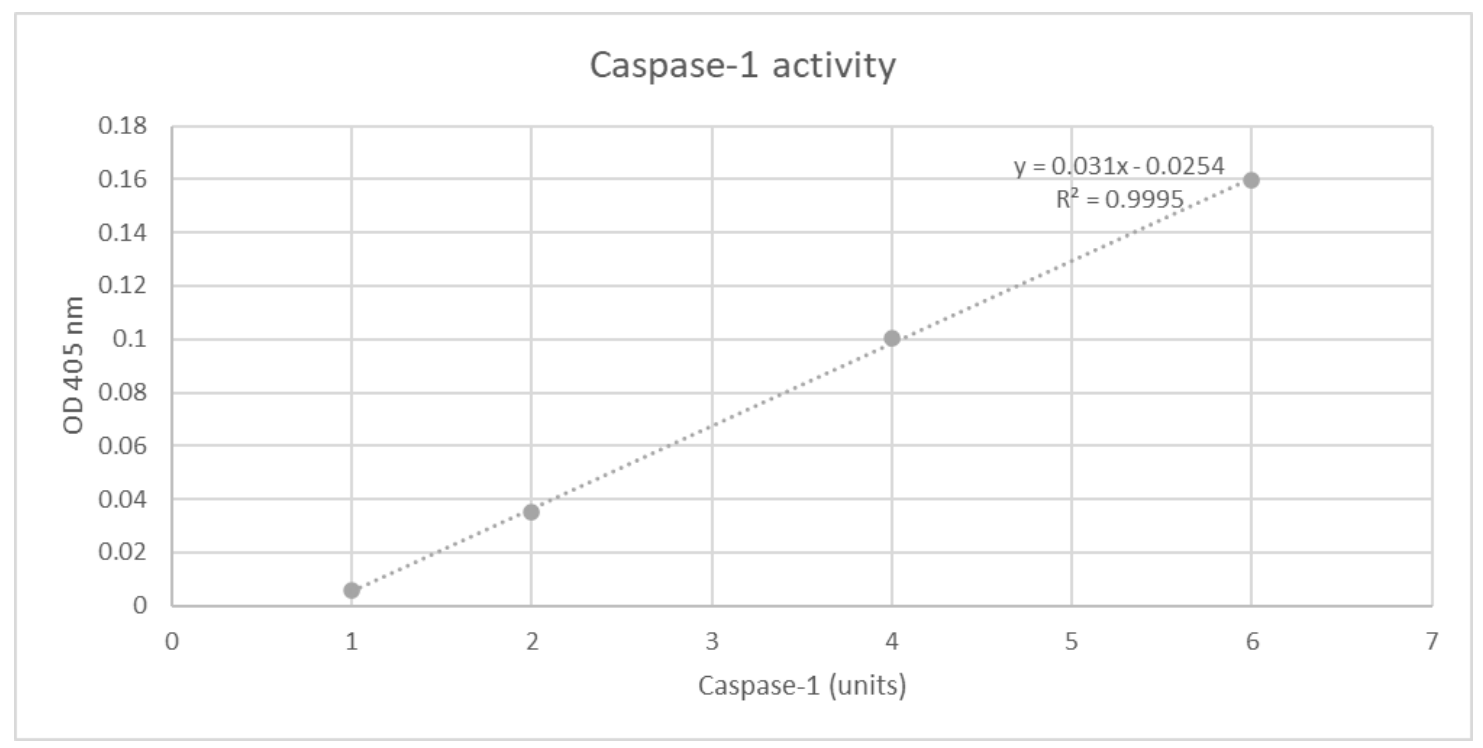

Figure C.1: Caspase-1 activity assay standard curve, using known Units of active recombinant Human caspase-1 (BioVision, \#1081-25) and the caspase-1/interleukin-1 converting enzyme (ICE) colorimetric assay (BioVision, \#K111)

Sample calculation of Caspase-1 Units:

WAT EC3 had OD 0.146 and the OD of the blank was 0.051

Blank subtracted OD ${ }_{\text {WAT EC3 }}=0.146-0.051=0.095$

$0.095+0.0254 / 0.031=\mathrm{x}=2.72$ units of caspase- 1 activity

For each condition, the units of caspase- 1 activity were averaged for all biological replicates and the standard error was calculated. 


\section{MicroRNA-sequencing library preparation}

Small RNA-sequencing library preparation was performed by the Canada's Michael Smith Genome Sciences Centre in British Columbia. Briefly, $8 \mu \mathrm{L}$ of total RNA was ligated to $2 \mu \mathrm{L}$ of a 2.5 $\mu \mathrm{M}$ 3' DNA adapter (5'-NNNNNNTGGAATTCTCGGGTGCCAAGTCG-3', where N = randomized sequence) in a 96-well microtitre plate. RNA secondary structure was denatured at $70{ }^{\circ} \mathrm{C}$ for 2 minutes and then snap chilled on ice. Then, $10 \mu \mathrm{L}$ of a ligation buffer containing truncated T4 RNA ligase $2(200 \mathrm{U} / \mu \mathrm{L}$, New England Biolabs) was added and the $20 \mu \mathrm{L}$ reaction was incubated for 1 hour at $22^{\circ} \mathrm{C}$. Purification RNA MagClean DX beads (Aline Biosciences) was done twice (at room temperature) to remove excess adapter. The 3' adapter-ligated RNAs were ligated to heat denatured 5' miRNA adapter (5'-UUCCCUACACGACGCUCUUCCGAUCUNNNNNN-3', N= randomized sequence) using T4 RNA ligase (5 U/ $\mu \mathrm{L}$, Ambion). This $20 \mu \mathrm{L}$ reaction was incubated for one hour at $37^{\circ} \mathrm{C}$. For first strand synthesis, the reverse transcriptase (RT) primer $\left(5^{\prime}\right.$ -

CGACTTGGCACCCGAGAATTCCA-3') was incubated with the ligated template at $65^{\circ} \mathrm{C}$ for 10 minutes before snap chilling on ice. Reverse transcription followed using Maxima H minus RT (200 $\mathrm{U} / \mu \mathrm{L}$ ) in a $40 \mu \mathrm{L}$ reaction incubated for one hour at $44{ }^{\circ} \mathrm{C}$. First strand cDNA was purified using an upper and lower bead clean-up (PCR Clean DX beads, Aline Biosciences) to remove excess RT primer and reduce non-target products prior to PCR enrichment. 15 cycles of PCR using one pairedend primer (PE 1.0, 5'AATGATACGGCGACCACCGAGATCTACACTCTTTCCCTACACGACGCTCTTCCGATCT-3') and miRNA indexed primers

(CAAGCAGAAGACGGCATACGAGATCNNNNNNATCGTACGTCGACTTGGCACCCGAGAA TTCCA; underlined index sequence is unique for up to 96 samples) was performed in a $50 \mu \mathrm{L}$ reaction volume incorporating Phusion Hot Start high fidelity DNA polymerase (NEB). A single paired-end primer was used for single-end sequencing, and the miRNA-indexed adapters were used to tag each sample with a different code for multi-sample sequencing. The amplified library was loaded onto a $12 \%$ PAGE gel and the region containing the miRNA library $(\sim 150 \mathrm{bp})$ was manually excised from the gel (size-selected). The size-selected library was ethanol precipitated and purified.

Using this method, mature miRNAs as well other small RNAs up to 30 nucleotides long are extracted from the $12 \%$ PAGE gel along with $\sim 120$ bp of adapter. Thus, downstream alignment steps are required to filter out sequences known to be non-miRNA small RNA sequences (e.g. piwi-RNA).

Quality control of the final library was performed using Qubit and Agilent DNA 1000 Series II assays prior to sequencing on an Illumina NextSeq500 instrument generating single-end 75 base reads. Since RNA molecules are fragile, quality control is done to ensure the samples are not degraded before sequencing.

A Qubit assay is a fluorometric approach that detects the concentration of dsDNA using a dye that emits fluorescence when bound to dsDNA. Small interfering molecules in the sample like salts, RNA and nucleotides do not get bound by the dye and therefore do not contribute to any overestimation of dsDNA concentration. Knowing the library concentration is required to ensure that each pooled library is sequenced to an appropriate read depth.

An Agilent DNA series II assay is used to ensure the abundance (miRNA concentration) and quality of RNAs of different sequence lengths. To calculate absolute amounts of RNAs of varying 
sizes, an RNA ladder (a mixture of 6 RNA transcripts of a well-defined size and total concentration) is run from the ladder well. For instance, to calculate the concentration of the RNA test sample, the area under the entire RNA electropherogram is determined and compared with the ladder area. Further, libraries should appear as a single peak on the electropherogram, so other peaks can identify if there are artifacts.

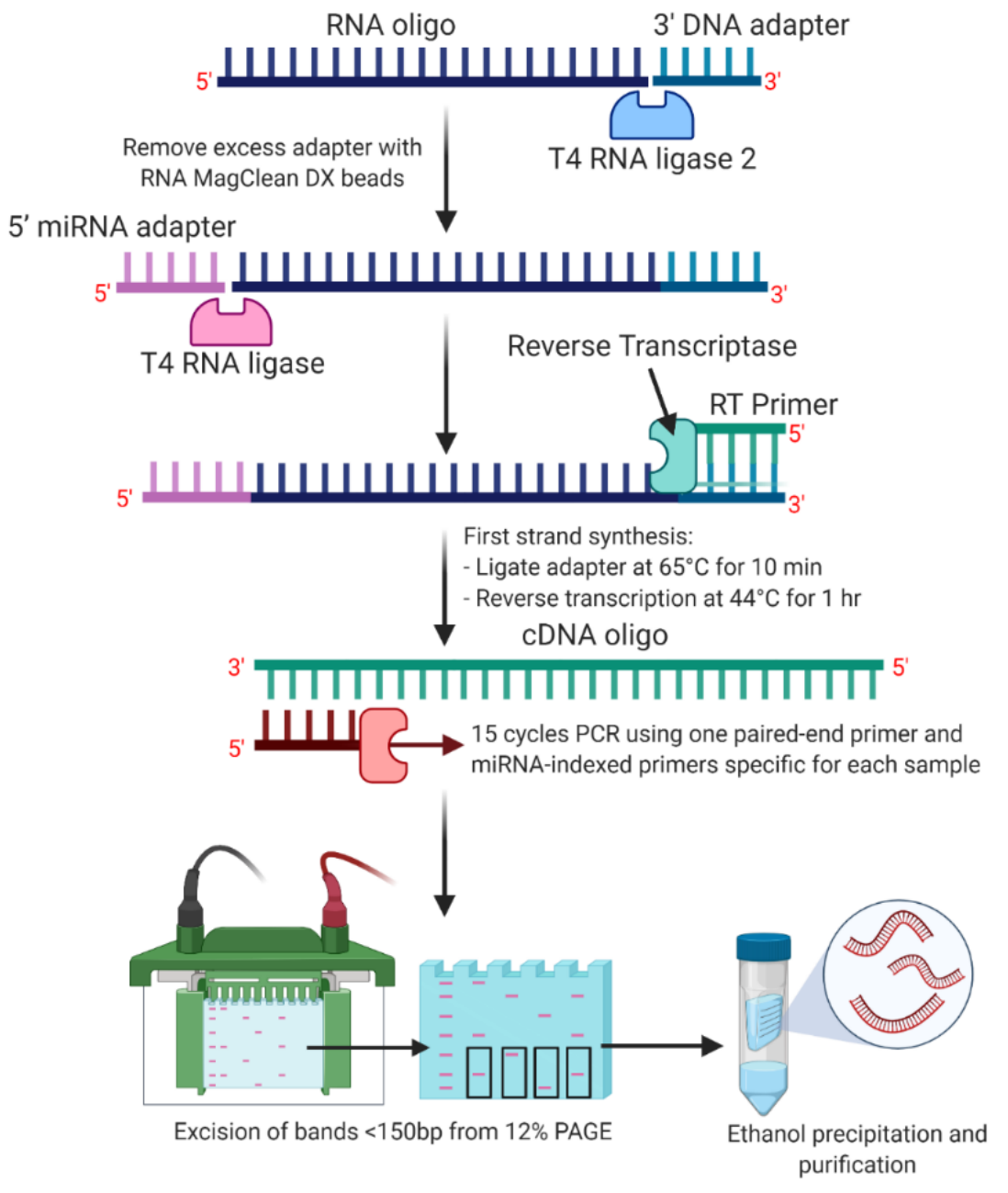

Figure D.1: Simplified workflow of adapter ligation, reverse transcription, PCR amplification, and purification of the cDNA library. 


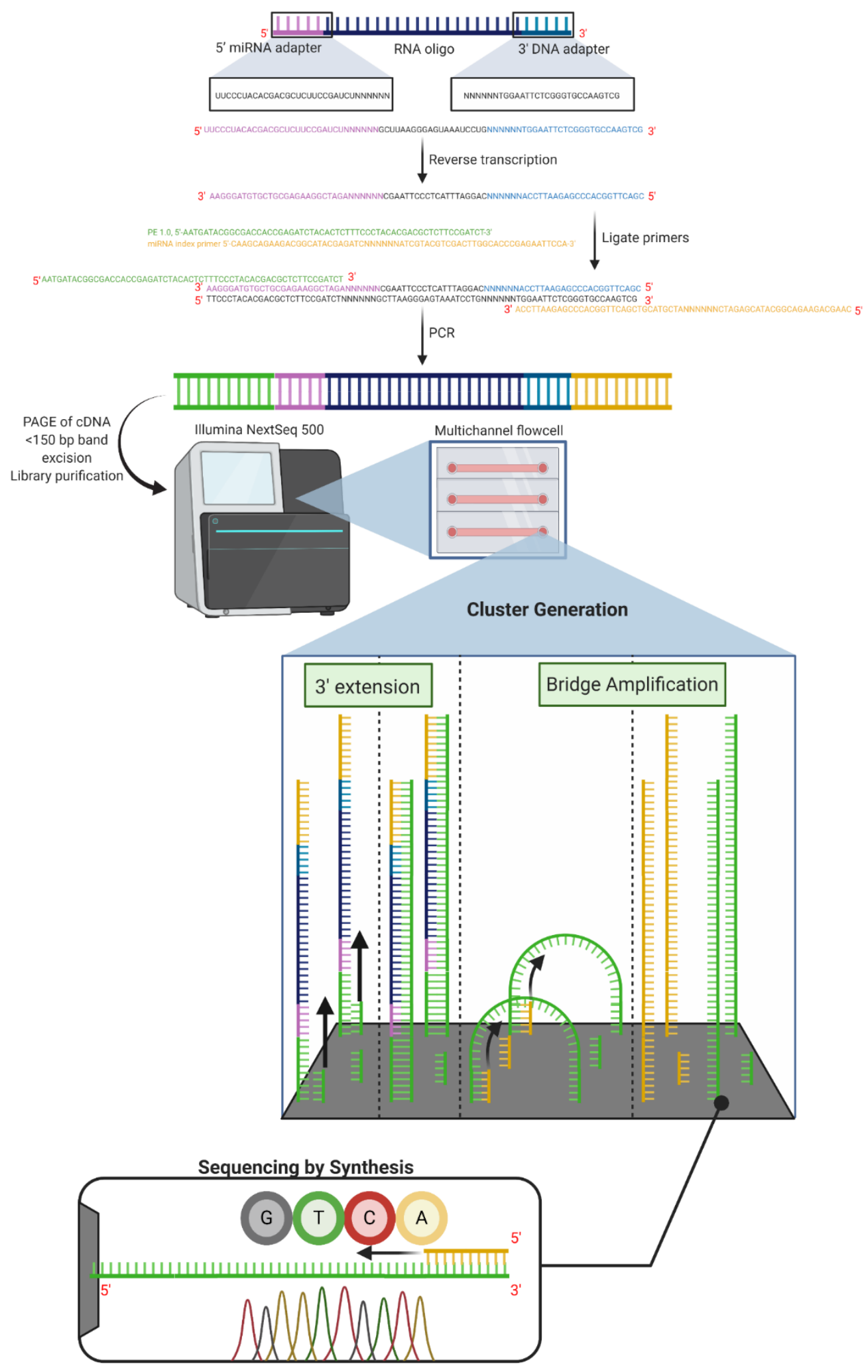

Figure D.2. Library preparation and Illumina NextSeq500 sequencing workflow 


\section{E. List of Publications}

${ }^{1}$ Equal Contribution

1. Logan SM and Storey KB. Submitted April 1, 2021. MicroRNA regulation of metabolically suppressed white adipose tissue in a wild fat-storing hibernator, Ictidomys tridecemlineatus. Molecular Genetics and Genomics.

2. Luu BE, Logan SM, Wang A, Levy RJ and Storey KB. Submitted. Isoflurane and low dose carbon monoxide modulates microRNAs involved in apoptosis signalling in the brains of postnatal mice. Neurotoxicology.

3. Logan SM and Storey KB. 2021 Mar 9. Markers of tissue remodeling and inflammation in the white and brown adipose tissues of a model hibernator. Cellular Signaling 82: June 2021, 109975. doi:10.1016/j.cellsig.2021.109975

4. Logan SM, Gupta A, Wang A, Levy RJ, Storey KB. 2021 Mar 4. Isoflurane and lowlevel carbon monoxide exposures increase expression of pro-survival miRNA in neonatal mouse heart. Cell Stress and Chaperones. Epub ahead of print. PMID: 33661504 doi: 10.1007/s12192-021-01199-0

5. Logan SM and Storey KB. 2021 Mar. MicroRNA expression patterns in the brown fat of hibernating 13-lined ground squirrels. Genomics 113:769-781. PMID: 33529780 doi:10.1016/j.ygeno.2021.01.017. Epub 2021 Jan 30.

6. Giroud S, Habold C, Nespolo RF, Mejías C, Terrien J, Logan SM, Henning RH, and Storey KB. 2020 Dec 21. The torpid state: Recent advances in metabolic adaptations and protective mechanisms. Front. Physiol. 11, 605186. PMID: 33192622. doi:10.3389/fphys.2020.623665.

7. Watts AJ, Logan SM, Kübber-Heiss A, Posautz A, Stalder G, Painer J, Gasch K, Giroud S, Storey KB. 2020 Dec 21. Regulation of Peroxisome Proliferator-Activated Receptor Pathway During Torpor in the Garden Dormouse, Eliomys quercinus. Frontiers in Physiology 11: 615025. PMID: 33408645 PMCID: PMC7779809 doi:10.3389/fphys.2020.615025

8. Logan SM and Storey KB. 2021 Jan. Inflammasome signaling could be used to sense and respond to endogenous damage in brown but not white adipose tissue of a hibernating ground squirrel. Dev Comp Immunol 114: 103819. doi:10.1016/j.dci.2020.103819. PMID: 32781003.

9. Logan SM, Watts AJ, Posautz A, Kübber-Heiss A, Painer J, Stalder G, Giroud S, Storey KB. 2020. The Ratio of Linoleic and Linolenic Acid in the Pre-hibernation Diet Influences NFkB Signaling in Garden Dormice During Torpor. Front Mol Biosci. 7:97. doi: 10.3389/fmolb.2020.00097. eCollection 2020.

10. Logan SM and KB Storey. 2020 Nov. Regrowth and neuronal protection are key for mammalian hibernation: Roles for metabolic suppression. Neural Regeneration Research 15: 2027-2028. doi:10.4103/1673-5374.282242

11. Logan SM and Storey KB. 2020 Nov. Cold-inducible RNA-binding protein Cirp, but not $\mathrm{Rbm} 3$, may regulate transcript processing and protection in tissues of the hibernating ground squirrel. Cell Stress Chaperones 25: 857-868 doi: 10.1007/s12192-020-01110-3.

12. Logan $\mathbf{S M}^{1}$, Szereszewski KE ${ }^{1}$, Bennett NC, Hart DW, van Jaarsveld B, Pamenter ME, and Storey KB. 2020 May. The brains of six African mole rat species show divergent 
responses to hypoxia. Journal of Experimental Biology 223: jeb215905. doi:10.1242/jeb.215905

13. Logan SM and Storey KB. Nov 18 2019. Angiogenic signaling in the lungs of a metabolically suppressed hibernating mammal (Ictidomys tridecemlineatus). PeerJ 7:e8116. doi:10.7717/peerj.8116. eCollection 2019.

14. Logan SM, Watts A, Storey KB. 2019. Brain dead: The dynamic neuroendocrinological adaptations during hypometabolism in mammalian hibernators. In: Ludwig, M., Levkowitz, G. (eds) Model Animals in Neuroendocrinology: From worm to mouse to man. John Wiley \& Sons Ltd., Ch. 9, pp. 207-231. ISBN: 978-1-119-39094-7

15. Logan SM, Wu C-W, Storey, KB. 2018. The squirrel with the lagging eIF2: global suppression of protein synthesis during torpor". Comp. Biochem. Physiol A 227: 161-171. PMID: 30343059

16. Ruberto AA, Logan SM, Storey KB. Sep 25 2018. Temperature and serine phosphorylation regulate glycerol-3-phosphate dehydrogenase in skeletal muscle of hibernating Richardson's ground squirrels. Biochem Cell Biol. 97: 148-157. doi:10.1139/bcb-2018-0198

17. Logan SM and Storey KB. 2018 June 4. Pro-inflammatory AGE-RAGE signaling is activated during arousal from hibernation in ground squirrel adipose. PeerJ 6: e4911. doi:10.7717/peerj.4911. eCollection 2018.

18. Logan SM ${ }^{1}$,Hadj-Moussa $\mathrm{H}^{1}$, Seibel BE, and Storey KB. 2018 June. Potential role for microRNA in regulating hypoxia-induced metabolic suppression in jumbo squids. $B B A$ Gene Regulatory Mechanisms 1861: 586-593. doi:10.1016/j.bbagrm.2018.04.007.

19. Logan SM and Storey KB. 2016 Sept. Tissue-specific response of carbohydrateresponsive element binding protein (ChREBP) to mammalian hibernation in 13-lined ground squirrels. Cryobiology 73:103-11. doi:10.1016/j.cryobiol.2016.09.002.

20. Logan SM and Storey KB. 2016 July. Avoiding apoptosis during mammalian hibernation (Comment on "Turn down genes for WAT? Activation of anti-apoptosis pathways protects white adipose tissue in metabolically depressed 13-lined ground squirrels" and "Response of the JAK-STAT pathway to mammalian hibernation in 13lined ground squirrel striated muscle"). Temperature 3: 1-3. doi:10.1080/23328940.2016.1211071

21. Logan SM, Luu BE, and Storey KB. 2016 May. Turn down genes for WAT? Activation of anti-apoptosis pathways protects white adipose tissue in metabolically depressed 13lined ground squirrels. Mol Cell Biochem 416(1-2):47-62. doi:10.1007/s11010-016-26950. Epub 2016 Mar 31

22. Logan SM, Tessier SN, Tye J, and Storey KB. 2016 Mar. Response of the JAK-STAT pathway to mammalian hibernation in 13-lined ground squirrel striated muscle. Mol Cell Biochem. 414(1-2):115-27. doi:10.1007/s11010-016-2665-6. Epub 2016 Feb 17. 


\section{F. List of Presentations}

1. Logan SM and Storey KB. Brown and White Adipose from Hibernators Sense and Respond to Inflammatory Triggers Differently. $22^{\text {nd }}$ Annual Chemistry and Biochemistry Graduate Research Conference, Montreal, Canada, November 15, 2019 (Talk)

2. Logan SM and Storey KB. Hibernator state of mind: the molecular adaptations of torpid brains. $10^{\text {th }}$ International Congress of Comparative Physiology and Biochemistry, Ottawa, Canada, August 5-9, 2019. (Invited Speaker)

3. Logan SM and Storey KB. Fat but fit: How hibernating ground squirrel adipose tissue regulates pro-inflammatory signaling pathways. $6^{\text {th }}$ Canadian Obesity Summit, Ottawa, Canada, April 23-26, 2019 (Poster)

4. Logan SM and Storey KB. The response of cold-shock RNA-binding proteins during hibernation in 13-lined ground squirrels. Serbian Society for Mitochondrial and Free Radical Physiology, Fourth International Congress, University of Belgrade, Serbia, September 28-30, 2018 (Poster)

5. Logan SM. Fat but Fit: How Hibernating Ground Squirrel Adipose Tissue Regulates Pro-inflammatory Signaling Pathways. 20th Annual Chemistry and Biochemistry Graduate Research Conference, Montreal, Canada, November 17, 2017 (Talk, ${ }^{\text {st }}$ prize in Molecular Biology/Biochemistry Division)

6. Logan SM and Storey KB. The response of cold-shock RNA-binding proteins during hibernation in 13-lined ground squirrels. Canadian Society for Molecular Biosciences (CSMB), Ottawa, Canada, May 16-20, 2017 (Poster)

7. Logan SM and Storey KB. The response of cold-shock RNA-binding proteins during hibernation in 13-lined ground squirrels. Ottawa-Carleton Institute of Biology (OCIB) Symposium, Ottawa, Canada, April 27-28, 2017 (Poster)

8. Logan SM and Storey KB. Response of the JAK-STAT pathway to mammalian hibernation in 13-lined ground squirrel. CRYO2016: The 53rd Annual Meeting of the Society for Cryobiology, Ottawa, Canada, July 23-27, 2016. (Talk)

9. Logan SM, Tessier S, Tye J, and Storey KB. Response of the JAK-STAT pathway to mammalian hibernation in 13-lined ground squirrel striated muscle. Ottawa-Carleton Institute of Biology (OCIB) Symposium, Ottawa, Canada, May 5-6, 2016 (Poster)

10. Logan SM and Storey KB. Turn down gene expression for WAT: Anti-apoptotic signaling protects white adipose tissue in hibernating ground squirrels. 18th Annual Chemistry and Biochemistry Graduate Research Conference, Montreal, Canada, November 20, 2015 (Poster)

11. Logan SM and Storey KB. Turn down gene expression for WAT: Anti-apoptotic signaling protects white adipose tissue in hibernating ground squirrels. 9th International Congress of Comparative Physiology and Biochemistry, Kraków, Poland, August 23-28, 2015. (Poster)

12. Logan SM and Storey KB. Turn down gene expression for WAT: Anti-apoptotic signaling protects white adipose tissue in hibernating ground squirrels. 2015 OttawaCarleton Institute of Biology (OCIB) Symposium, Ottawa, Canada, April 29-30, 2015 (Poster)

13. Logan SM and Storey KB. Turn down gene expression for WAT: Anti-apoptotic signaling protects white adipose tissue in hibernating ground squirrels. Faculty of Science Research Day 2015, Ottawa, Canada, April 10, 2015 (Poster, ${ }^{\text {st }}$ prize) $^{-}$ 
14. Logan SM and Storey KB. Turn down gene expression for WAT: Anti-apoptotic signaling protects white adipose tissue in hibernating ground squirrels. Ontario Biology Day, Ottawa, Canada, March 21-22, 2015 (Poster) 